\title{
Design of High-Ionic Conductivity Electrodes for Direct Methanol Fuel Cells
}

by

\section{Anthony J. Schrauth}

B.S., Tufts University (2003)

M.S., Massachusetts Institute of Technology (2005)

Submitted to the Department of Mechanical Engineering MASSACHUSETTS INSTITUTE OF TECHNOLOGY

JUL 292011 in partial fulfillment of the requirements for the degree of

Doctor of Philosophy in Mechanical Engineering

at the

\section{MASSACHUSETTS INSTITUTE OF TECHNOLOGY}

June 2011

(C) Massachusetts Institute of Technology 2011. All rights reserved.
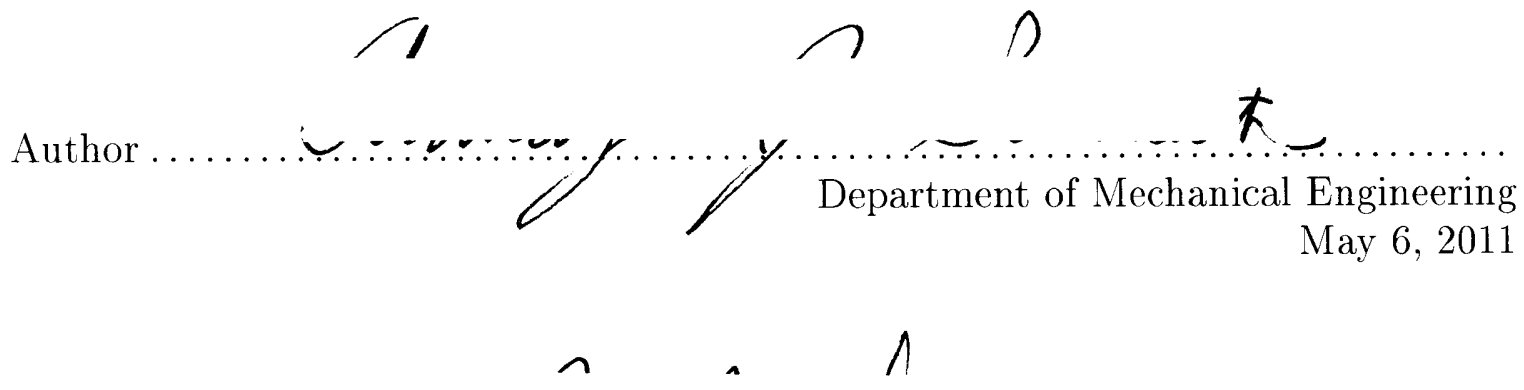

Certified by<smiles>C1CCCC1</smiles>

Jung-Hoon Chun Professor, Department of Mechanical Engineering Director, Laboratory for Manufacturing and Productivity Thesis Supervisor

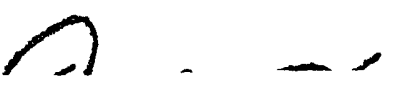

Accepted by

David Hardt

Chairman, Departmental Committee on Graduate Studies Ralph E. \& Eloise F. Cross Professor of Mechanical Engineering 


\title{
Design of High-Ionic Conductivity Electrodes for Direct Methanol Fuel Cells
}

\author{
by \\ Anthony J. Schrauth \\ Submitted to the Department of Mechanical Engineering \\ on May 6, 2011, in partial fulfillment of the \\ requirements for the degree of \\ Doctor of Philosophy in Mechanical Engineering
}

\begin{abstract}
Carbon-supported porous electrodes are used in low-temperature fuel cells to provide maximum catalyst surface area, while taking up little volume and using minimum catalyst material. In Direct Methanol Fuel Cells (DMFCs), however, much of the catalyst included in the anode is significantly under-utilized, while a small fraction of the catalyst facilitates the bulk of the oxidation reaction.

In this thesis, the porous carbon electrode used as the anode in a DMFC is analyzed using Axiomatic Design theory. The imbalance of catalyst utilization in these electrodes is determined to be a result of coupled design, in which large amounts of catalyst can compromise ionic resistance and fuel transport within the electrode. This design flaw is confirmed experimentally using cyclic voltammetry and impedance spectroscopy. Tests of standard electrodes show that they have a maximum Nafion content of about $30 \%$ Nafion by weight and that excessive catalyst loading eventually results in less available catalyst, not more. An alternative design is proposed to alleviate the coupling between functions by applying micron-scale structure to the nano-porous electrode. The proposed design introduces ionically conductive channels through the thickness of the porous electrode to greatly reduce ionic resistance to catalyst particles far from the ion exchange membrane without compromising access to catalyst particles near the membrane accessible for fuel delivery and product removal.

The influence of the proposed design on ionic conductivity is analyzed using a twodimensional analog of the transmission line model for porous electrodes. The model suggests that ionic resistance can be decreased by up to $87 \%$ with the addition of ionically conductive posts. Structured electrodes with $75 \mu$ m diameter posts spaced $175 \mu \mathrm{m}$ apart are shown in electrochemical impedance spectroscopy experiments to perform notably better than standard cells. The structured cells show a $6 \%$ increase in available catalyst area and a $46 \%$ decrease in ionic resistance. Peak cell power is estimated to increase by $4 \%$ as a result of the best electrode tested while an electrode with ideal geometry could increase peak cell power by $9 \%$. Even greater benefits could be realized if, as predicted, structured cells can keep ionic resistance constant while catalyst loading is increased.
\end{abstract}

Thesis Supervisor: Jung-Hoon Chun

Title: Professor, Department of Mechanical Engineering

Director, Laboratory for Manufacturing and Productivity 


\section{Acknowledgments}

Through my years, I have been fortunate to meet, befriend, and work with numerous outstanding people. It is these interactions and friendships that will provide me with lasting strength and the realization that happiness and satisfaction are driven by people and relationships, not places or things. The people mentioned below are but a selection, hopefully not a random one, of the people who helped me through the entire $\mathrm{PhD}$ experience.

First, I would like to thank my advisor, Professor Jung-Hoon Chun. He was kind enough to take me in as an advisee early on in this project and guide me as I developed an abstract idea into a concrete contribution. I am also indebted to him for giving me the opportunity to TA 2.008, which was one of the most enjoyable academic experiences I had at MIT. I would also like to thank my thesis committee members, Professor Sang-Gook Kim and Professor Yang Shao-Horn. Both were generous with their time and provided valuable feedback and guidance as I developed this thesis. Their assistance, both technical and non-technical, allowed me to grow, learn, and make the most out of my time at MIT.

I would also like to thank my first advisor at MIT, Professor Nam Pyo Suh, for hiring me when I first got to MIT and for believing in my abilities ever since. His belief in the centrality of design to the innovation process and his fearless and prolific production of "crazy" ideas has been an inspiration to me.

I was fortunate to befriend doctor Nannaji Saka in my time at MIT. He was always happy to provide a friendly ear and is never short on advice for handling life's little curve balls.

I would like to thank the instructors in the LMP machine shop: Bill Buckley, Dave Dow, and Pat McAtamney. They and the shop they run also provided a welcome refuge from the doubts and frustrations that occasionally threatened to derail progress on my experimental work.

I am greatly indebted to David Liu for graciously allowing me to take his time and use the impedance spectroscopy setup in his lab to gather most of the data used to support this thesis. More than access to equipment, David was always happy to discuss the various minutia of my evolving experimental challenges and simply having him there to listen was an immense help. Without his valuable feedback and insightful questions I would not have been able to gather and interpret the data on my cells. 
I would like to thank the Korean Institute for Machinery and Materials for their financial support at the beginning of this project. I would also like to thank the Singapore University of Technology and Design for stepping in to fund my work at the end of my time at MIT.

In addition to the technical assistance directly related to my studies at MIT, a vast number of people have helped support me with their friendship and caring. My parents, Chris and Peter Schrauth, have always been there for me with their love and encouragement and for that I am eternally grateful. My mother- and father-in-law, Pat and Dick Norris, treated me like a son long before I had earned such kindness. My siblings, Tim, Sam, and Hilary, have always provided me with a source of pride in all that they do and I am grateful for the support.

While at MIT I have been fortunate to meet a number of amazing people, who I am proud to call my friends. My labmates over the years, Hrishi Deo, Steve Bathurst, Thor Eusner, Eehern Wong, Vu Hong, Peter Jeziorek, Beto Peliks, Ishan Barman, Catherine Mau, Ryan Slaughter, Nikil Padhye, and Aron Blaesi have made my time studying at MIT much more rewarding than it would have been without them. In addition to my labmates, I have been fortunate to have studied in the LMP, the home of the coolest graduate students around, and have greatly enjoyed the friendship and antics of Michael Cullinan, Melinda Hale, Aaron Mazzeo, Matt Dirckx, Dieter Brommer, Anujli Appapillai, Bob Panas, Chris Dibiasio, Sarah Bernardis, Joe Petrazelka, Maria Telleria, and more.

In a place like MIT, breaking out of one's academic circle can provide a welcome breath of fresh air and for that breath was provided by the MIT Cycling Team. The friends I've met through the team are far too numerous to list, but they are among the most cherished from my time at MIT. There are many more friends that have been a source of much of my happiness over the years and despite not being able to name them all here I am eternally grateful for all the memories they have provided me with and I look forward to many more ahead.

Most of all, I thank my wife, Susan, for joining me on this journey and keeping me going no matter how desperately I wanted to stop. Without her love and support, nothing I have achieved would have been possible. 


\section{Contents}

$\begin{array}{lll}1 & \text { Introduction } & 17\end{array}$

2 Background $\quad 23$

2.1 Fuel Cell Fundamentals . . . . . . . . . . . . . . . . . . . . . . 23

2.1.1 Direct Methanol Fuel Cells . . . . . . . . . . . . . . . 24

2.1.2 Sources of Inefficiency in Fuel Cell Systems . . . . . . . . . . . 26

2.1 .3 Electrode Structure . . . . . . . . . . . . . . . 27

2.2 History of porous fuel cell electrodes . . . . . . . . . . . . . 28

2.2 .1 Early porous electrodes . . . . . . . . . . . . . . 28

2.2 .2 Ionomer Impregnated Electrodes . . . . . . . . . . . . . . 29

2.2 .3 Ionomer Based Inks . . . . . . . . . . . . . . . . . . . 29

2.3 Ionic Resistance in Fuel Cell Electrodes . . . . . . . . . . . . . . . . . . . . 29

2.3.1 Optimum Nafion Loading . . . . . . . . . . . . . . 30

2.3.2 Catalyst Loading Limit . . . . . . . . . . . . . . . . . 32

2.4 Previous Structured Electrodes . . . . . . . . . . . . . . . 32

3 Design and Analysis of Structured Electrodes 35

3.1 Axiomatic Design Analysis of Porous Electrodes . . . . . . . . . . . . . 35

3.1 .1 Axiomatic Design Theory . . . . . . . . . . . . . 35

3.1.2 Conventional Porous Electrode Decomposition . . . . . . . . . . 37

3.2 Axiomatic Design Inspired Solution . . . . . . . . . . . . . . . . . . 39

3.3 Transmission Line Model for a DMFC Anode . . . . . . . . . . . . . . . . . . 41

3.3 .1 Applications to Impedance Spectroscopy . . . . . . . . . . . . . . 48

3.3 .2 Constant Phase Element . . . . . . . . . . . . . . . . . 52

3.3 .3 Catalyst Loading Limit . . . . . . . . . . . . . . . . 55 
3.4 Model of Structured Fuel Cell Electrodes . . . . . . . . . . . . . . . . . . . 57

3.4.1 Two-Dimensional Model Architecture . . . . . . . . . . . . . . . . . . 59

3.4.2 Structured Model Results . . . . . . . . . . . . . . . . . . . . 62

3.4.3 Effect of Material Properties _. . . . . . . . . . . . . . . . . 64

3.4.4 Effect of Structured Electrode Geometry . . . . . . . . . . . . 67

3.4.5 Utility of the Two-Dimensional Structured Model . . . . . . . . . . . . 83

3.5 Summary . . . . . . . . . . . . . . . . . . . 84

4 Experimental Methods and Procedures $\quad 87$

4.1 Cyclic Voltammetry . . . . . . . . . . . . . 87

4.1 .1 Electrochemically Active Area . . . . . . . . . . . . . 88

4.1 .2 Electrode Ionic Resistance . . . . . . . . . . . . . . . . . . . 89

4.2 Impedance Spectroscopy . . . . . . . . . . . . . . . . . 91

4.2.1 Capacitance Plots of Impedance Data . . . . . . . . . . . . . 95

4.2.2 Transmission Line Data Fitting . . . . . . . . . . . . . . . . . . . 95

4.3 Cell Setup . . . . . . . . . . . . . . . . . . . . . . . . . . . . .

4.3.1 Three-Electrode Cell with Aqueous Electrolyte . . . . . . . . . 97

4.3.2 Solid Electrolyte Impedance Cells . . . . . . . . . . . . . . . . . . . 99

4.4 Electrode Fabrication . . . . . . . . . . . . . . . . . . . . 101

4.4 .1 Catalyst Ink . . . . . . . . . . . . . . . 101

4.4.2 Glassy Carbon Electrodes . . . . . . . . . . . . . . . . 102

4.4 .3 Through-Plane Electrodes . . . . . . . . . . . . . . . . 104

5 Results and Discussion $\quad 113$

5.1 Influence of Nafion Content on Catalyst Availability . . . . . . . . . . 113

5.1.1 Determination of Ideal Nafion Loading with Cyclic Voltammetry . . . 113

5.1.2 Impedance Spectroscopy with Changing Nafion Content . . . . . . . 115

5.2 Influence of Catalyst Loading on Ionic Resistance . . . . . . . . . . . . 118

5.2.1 Effect of Catalyst Loading with Cyclic Voltammetry . . . . . . . 118

5.2.2 Catalyst Loading Changes in Impedance Spectroscopy . . . . . . . . 122

5.2 .3 Catalyst Loading Limit _ . . . . . . . . . . . . . . . 125

5.3 Performance of Structured Cells . . . . . . . . . . . . . . . 128

5.3.1 Influence of Structured Electrodes on Catalyst Loading Limits . . . . . 135 
6 Conclusions $\quad 141$

6.1 Contributions . . . . . . . . . . . . . . . . . . 148

6.2 Future Work . . . . . . . . . . . . . . . . . . . . 148

A First Generation Solid Electrolyte Cells $\quad 151$

A.1 Fabrication of First Generation Solid Electrolyte Cells . . . . . . . . . . 152

A.1.1 Rigid Current Collector Impedance Fixture . . . . . . . . . . . . 152

A.1.2 Ink-Based Counter Electrode Cells . . . . . . . . . . . . . . . . . . . 152

A.2 Impedance Spectroscopy Results with First Generation Solid Electrolyte Cells 155

A.2.1 Resolution of Electrode Resistance . . . . . . . . . . . . . . 155

A.2.2 First Structured Cells _ . . . . . . . . . . . . . . . 157

A.2.3 Cell Variations . . . . . . . . . . . . . . . . . . . . . 160

A.2.4 Solid Electrolyte Cells with Carbon Paper Electrodes . . . . . . . . 161

B Supplementary Results $\quad 165$

B.1 Cyclic Voltammetry of Structured Electrodes . . . . . . . . . . . 165

B.2 Impedance Spectroscopy with Liquid Electrolyte Cells . . . . . . . . . . 167

B.2.1 Structured Electrodes Cast on Carbon Paper . . . . . . . . . . 168

B.2.2 Nafion loading in Standard Electrodes . . . . . . . . . . . 171

B.2.3 Impedance Spectroscopy of Bare Glassy Carbon Supports . . . . . . 171 


\section{List of Figures}

1-1 Rough comparison of battery and fuel cell mass as a function of energy capacity assuming $20 \%, 35 \%$, and $50 \%$ efficient DMFCs. . . . . . . . . 19

2-1 Direct methanol fuel cell schematic. . . . . . . . . . . . . . . . . 24

2-2 Schematic representation of a portion of a typical DMFC anode structure. . 27

2-3 Polarization curves for DMFCs with several anode Nafion loadings. Taken from Zhao et al $[1] . \ldots \ldots \ldots \ldots \ldots \ldots \ldots$

2-4 Polarization (a) and power density (b) plots for different catalyst loadings. Taken from Shimizu et al $[2] \ldots \ldots$. . . . . . . . . . . . . . . 33

3-1 Diagram of Nafion post electrode. . . . . . . . . . . . . . . . 41

3-2 Diagram of the network electrode model showing the two separate conduction regimes connected through a non-linear faradaic process. . . . . . . . . . . 42

3-3 Exchange current as a function of position in the electrode as simulated with network model with $x=0$ corresponding to the current collector. . . . . . . 42

3-4 Overpotenial as a function of position in the electrode as simulated with network model with $x=0$ corresponding to the current collector. . . . . . . 43

3-5 Differential section of a transmission line. . . . . . . . . . . . . 45

3-6 Calculated Nyquist plots of a transmission line model shown with (a) the $R \cdot l / 3$ real limit; (b) several $R$ values; (c) several $C$ values; and (d) several $L$ values. $\quad 50$

3-7 Calculated capacitance plots of a transmission line model shown with (a) the $R \cdot l / 3$ real limit and $C \cdot l$ capacitance limit; (b) several $R$ values; (c) several $C$ values; and $(\mathrm{d})$ several $L$ values . . . . . . . . . . . . . . . . . 51

3-8 Example cell illustrating the origins of the constant phase element. . . . . . . 52 
3-9 Calculated Nyquist plots of a transmission line model using constant phase element charge transfer and shown with (a) several $\phi$ values; (b) several $R$ values; (c) several $C$ values; and (d) several $L$ values . . . . . . . . . . . . . . 54

3-10 Calculated capacitance plots of a transmission line model using constant phase element charge transfer and shown with (a) several $\phi$ values; (b) several $R$ values; (c) several $C$ values; and (d) several $L$ values . . . . . . . . . . . . . . 56

3-11 Top and side views of the structured electrode showing lines of symmetry and the resulting unit cell of the post pattern. . . . . . . . . . . . . 58

3-12 Node structure used in two-dimensional model . . . . . . . . . . . . . 60

3-13 Nyquist (a) and capacitance (b) plots comparing standard and structured electrodes. . . . . . . . . . . . . . . . . . . 63

3-14 Nyquist (a) and capacitance (b) plots comparing standard and structured

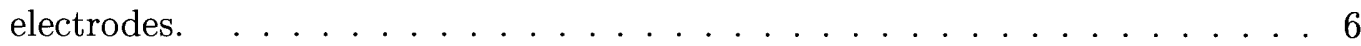

3-15 Capacitance plot showing the effect of changing capacitance density within the electrode material on the impedance spectra predicted from the structured electrode model. . . . . . . . . . . . . . . . . . 66

3-16 Nyquist (a) and capacitance (b) plots showing the effect that electrode ionic resistivity has on the behavior of the structured electrode model. . . . . . . . 68

3-17 Ionic resistance value for changing electrode ionic resistivity. . . . . . . . . 69

3-18 Nyquist (a) and capacitance (b) plots showing the effect that electrode ionic resistivity has on the behavior of the structured electrode model. . . . . . . 70

3-19 Ionic resistance value for changing structured electrode post diameters. . . . 71

3-20 CPE capacitance value for changing structured electrode post diameters. . . 72

3-21 Nyquist plots showing the effect that electrode thickness has on the behavior of the structured electrode model. . . . . . . . . . . . . . . . 74

3-22 Ionic resistance value for changing electrode thickness. . . . . . . . . . . 75

3-23 Nyquist (a) and capacitance (b) plots showing the effect that Nafion post spacing has on the behavior of the structured electrode model. . . . . . . . 76

3-24 Nyquist (a) and capacitance (b) plots showing the effect of changing the Nafion post diameter while keeping the ratio of post diameter to post spacing

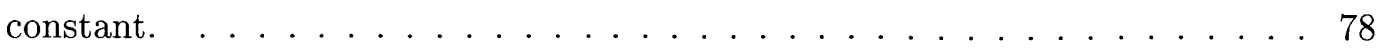

3-25 Effect of post spacing ratio, $d / w$, on ionic resistance. . . . . . . . . 79 
$3-26$ Effect of post spacing ratio, $d / w$, on capacitance. . . . . . . . . . 80

3-27 Effect of gap aspect ratio on ionic resistance. . . . . . . . . . . . . 81

3-28 Effect of gap aspect ratio on capacitance. . . . . . . . . . . . . . 82

4-1 A cyclic voltagram of a platinum disc illustrating the use of hydrogen adsorption area to measure the active platinum area using the adsorbed hydrogen method. The area between the voltagram and the constant double-layer charging current, shown in red, is proportional to the electrochemically active area of platinum. . . . . . . . . . . . . . . . . . . 90

4-2 Voltagram of standard electrode and an identical electrode encased within a layer of Nafion. . . . . . . . . . . . . . . . . . . . . 91

$4-3$ Randles equivalent circuit. . . . . . . . . . . . . . . . 92

4-4 Nyquist plot of a Randles Equivalent Circuit with $R_{s}=100 \Omega, R_{c t}=200 \Omega$, $C=10 \mu \mathrm{F}$, and $\sigma=200 \Omega / \sqrt{\mathrm{s}} \ldots \ldots \ldots \ldots \ldots \ldots$. . . . . . . . . . . . . . . . .

4-5 Capacitance plots of standard electrode data and best fit with a modified transmission line. . . . . . . . . . . . . . . 96

4-6 Photograph of the three-electrode cell with aqueous electrolyte. . . . . . 9 98

4-7 Second generation of solid electrolyte impedance cell. . . . . . . . . . . . 100

4-8 Cast Nafion posts on carbon paper with the focal plane at the top of the posts. 105

4-9 Cast Nafion posts on carbon paper with the focal plane at the bottom of the

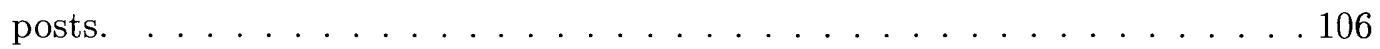

4-10 Diagrams of fabrication challenges for structured cells with carbon paper counter electrodes. . . . . . . . . . . . . . . . . . . . . . 109

4-11 Diagram of the stepped mold and foil combination used to emboss structured electrodes that use carbon paper counter electrodes. . . . . . . . . . . . 110

4-12 Process used to fabricate structured cells with catalyzed carbon paper counter electrodes.

5-1 Voltagram of standard cells with varied Nafion content all with $0.3 \frac{\mathrm{mg}}{\mathrm{cm}^{2}}$ catalyst loading. . . . . . . . . . . . . . . . . . . 114

5-2 Plot of electrochemically active catalyst area as Nafion loading of the electrode is changed. . . . . . . . . . . . . . . . . . . . . . . . . . . . . . . . 
5-3 Nyquist plots measured using catalyzed carbon paper counter electrodes showing the influence of working electrode Nafion loading. . . . . . . . . 116

5-4 Capacitance plot of standard cells with changing Nafion content. . . . . . . 117

5-5 Voltagram of standard cells with varied catalyst loading . . . . . . . 119

5-6 Voltagram of standard cells with varied catalyst loading that is normalized to measured active platinum area. . . . . . . . . . . . . 120

5-7 Plot of electrochemically active catalyst area as catalyst loading in the electrode is changed. . . . . . . . . . . . . . . . . . 121

5-8 Nyquist plots of cells with high and low Nafion content and high and low catalyst content. . . . . . . . . . . . . . . . . . . 123

5-9 Capacitance plot of cells with high and low Nafion content and high and low catalyst content. . . . . . . . . . . . . . . . . . . 124

5-10 Capacitance plot of standard cells as catalyst loading is increased. . . . . . 126

5-11 Best fit capacitance as a function of catalyst loading for standard ink compo-

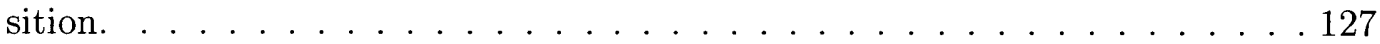

5-12 Capacitance plot of standard cells with low-Nafion ink as catalyst loading is increased. . . . . . . . . . . . . . . . . . 128

5-13 Best fit capacitance as a function of catalyst loading for standard ink composition. . . . . . . . . . . . . . . . . . . . . 129

5-14 Capacitance plot comparing structured and standard electrodes created with $9.1 \%$ Nafion content. . . . . . . . . . . . . . . . . 130

5-15 Capacitance plot comparing structured and standard electrodes created with $28.6 \%$ Nafion content.

5-16 Capacitance plot of structured electrodes with standard ink.

5-17 Capacitance plots calculated from the average best-fit parameters of structured and unstructured electrodes made with $60 \mu \mathrm{L}$ of standard ink. The spectra have been normalized to a single series resistance value. . . . . . . 134

5-18 Capacitance plots calculated from the average best-fit parameters of structured and unstructured electrodes made with $60 \mu l$ of low-Nafion ink. . . . 136

5-19 Capacitance plots of structured and standard electrodes made with $80 \mu l$ standard ink. . . . . . . . . . . . . . . . . . . . . . . . . . . . . .

5-20 Capacitance plots of low-Nafion electrodes with increasing catalyst loading. . 139 
6-1 Estimated impact of structured electrodes on full cell polarization. . . . . . . 144

6-2 Estimated impact of structured electrodes on full cell power density. . . . . . 145

6-3 Impact of decoupled design on maximum cell power. . . . . . . . . . . . . 147

A-1 Proposed impedance cell setup . . . . . . . . . . . . . . . . 151

A-2 Process used to fabricate structured cells with ink-based counter electrodes. . 153

A-3 First Samples with Solid Electrolyte . . . . . . . . . . . . . 155

A-4 Parallel resistor-capacitor equivalent circuit. . . . . . . . . . . . 156

A-5 Nyquist plot of a structured and a standard electrode both with ink-based counter electrodes. . . . . . . . . . . . . . . . . . . 157

A-6 Randles equivalent circuit diagram. . . . . . . . . . . . . . 158

A-7 Nyquist plot of cells fabricated to check cell-to-cell variation. . . . . . . . 159

A-8 Nyquist plot of cells with carbon paper counter electrodes. . . . . . . . . 162

B-1 Voltagram of a structured electrode and a standard electrode. . . . . . . 166

B-2 Nyquist plot of standard and structured electrodes measured in the liquid electrolyte cell. . . . . . . . . . . . . . . . . . . . 169

B-3 Bode plot of standard and structured electrodes measured in the liquid electrolyte cell. . . . . . . . . . . . . . . . . . . . 170

B-4 Nyquist plot of standard electrodes with a range of Nafion content taken using liquid electrolyte. . . . . . . . . . . . . . . . . . 172

B-5 Nyquist plot of impedance for all six glassy carbon electrode supports. . . . . 173

B-6 Zoomed view of the Nyquist plot of impedance for all six glassy carbon electrode supports. . . . . . . . . . . . . . . . . . . . 174 


\section{Chapter 1}

\section{Introduction}

Very little of the world's energy use is consumed in the same form in which it entered one of the many human-created energy distribution systems. That is, the productive use of energy almost always requires the conversion of that energy from one form to another, be that the refining of oil into gasoline or the conversion of alternating current from an outlet to direct current used to charge a cell phone. The ability to convert energy efficiently from one form to another is essential to our modern use and distribution of energy. The conversion of chemical energy stored in molecular bonds to electrical energy takes place in many different situations and scales, ranging from power plants to watch batteries. Each application utilizing such a conversion has different requirements and constraints, suggesting the need to broad suite of conversion methods, of which fuel cells are one.

Fuel cells are electrochemical conversion devices that use heterogeneous reactions to convert chemical energy in molecular bonds to DC electrical current without any intermediate steps. Since every conversion step has finite inefficiency, the elimination of intermediate steps between the chemical domain and the desired electrical domain can lead to better overall conversion efficiency. Fuel cells have found applications in off-grid combined heat and power generation, emergency backup, space systems, and solar power storage, among others. There are many types of fuel cells, which are distinguished by the type of electrolyte used, the fuel used, and the operation temperature. Reviews of the various types of fuel cells are available in several texts [3,4]. Each type of fuel cell has it's own strengths that suit it particularly well for certain applications. In this thesis, low-temperature Proton Exchange Membrane Fuel Cells (PEMFCs) using methanol fuel are considered. These cells are 
referred to as Direct Methanol Fuel Cells (DMFCs), since they oxidize methanol directly, without first reforming it into hydrogen. A more detailed explanation of their operation will be presented in Chapter 2. The main advantage that DMFCs have over hydrogen-fueled PEMFCs is that the storage of methanol, a liquid at room temperature, is much easier than the storage of hydrogen.

Direct Methanol Fuel Cells are often proposed as the next generation portable power source that will replace lithium-ion batteries $[3,5]$ for certain applications. This bold prediction for the displacement of lithium-ion batteries is not without merit. A fuel cell is an open system in which the fuel is fed into the cell, reacted to generate power, and products are exhausted from the cell. Batteries are closed systems, meaning the reaction products and reactants must all be contained within the cell. One of the major benefits of this arrangement is that the power and the total energy of a cell can be specified independently. In fuel cells, the power is determined by the type and number of cells used. Once the desired power can be reached, there is no need to add additional cells. The total energy of the system can then be adjusted independently by changing the volume of fuel stored separately from the actual cells. As more fuel is added to the system, the energy density of the system approaches the energy density of the fuel. For a DMFC, the energy density of the fuel is $5.54 \mathrm{kWh} / \mathrm{kg}$ [3]. The mass of complete fuel cell system, $M_{F C}$ is:

$$
M_{F C}=M_{P}+\frac{E}{\gamma \epsilon}
$$

where, $M_{p}$ is the mass of the actual cells and balance of plant components needed to meet the desired power output, $E$ is the total energy capacity required, $\gamma$ is the energy conversion efficiency, and $\epsilon$ is the energy density of the fuel.

In the case of a battery, the power is also increased by adding cells to the system. The total energy, however, is also controlled by the cell design. If more total energy is needed, it must be added with either additional cells or changes to the existing cell electrodes to store more energy. As a consequence, the energy density of the whole system is roughly constant for any desired total energy stored. Typical lithium-ion batteries have an energy density of $180 \mathrm{Wh} / \mathrm{kg}$ [6]. The mass of a lithium-ion battery system varies with energy stored in a linear fashion with a slope of $1 / 180 \mathrm{~kg} / \mathrm{Wh}$. Compare that with a fuel cell's system mass, which is also linear in $E$, but with a slope of $1 / \gamma \epsilon$. Even for a cell with only $35 \%$ efficiency, the slope 


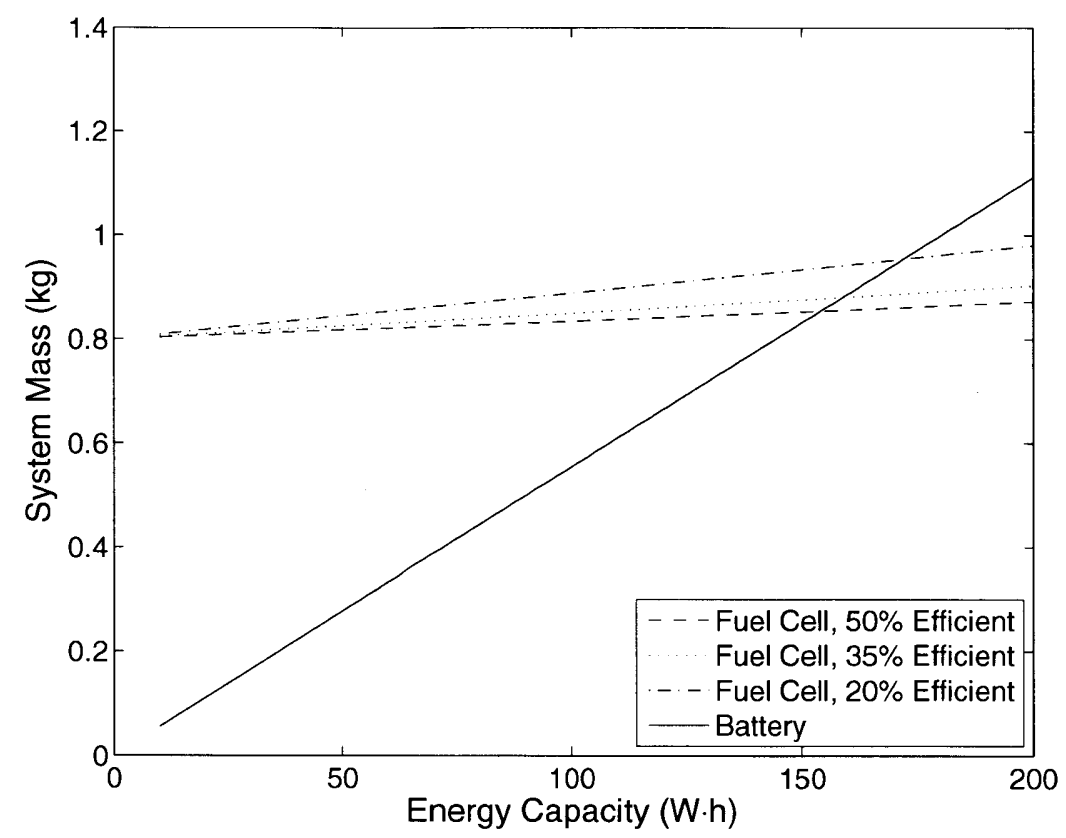

Figure 1-1: Rough comparison of battery and fuel cell mass as a function of energy capacity assuming $20 \%, 35 \%$, and $50 \%$ efficient DMFCs.

of the fuel cell mass curve would be an order of magnitude lower than that of the lithium ion battery system. An example assuming a $35 \%$ efficient fuel cell with a cell mass of $0.8 \mathrm{~kg}$ is shown in Figure 1-1. As a reference, typical laptop batteries have an energy capacity of 50 to $100 \mathrm{~W} \cdot \mathrm{h}$.

The implications of this are two-fold. First, if the application requires long run-time and low power, then using a methanol fuel cell in place of a lithium ion battery become advantageous. Second, and perhaps more important, in order for fuel cells to compete with lithium ion batteries in a broad range of portable power applications, the power density of the individual cells must be improved, as this has a strong influence on the where the crossover point is between fuel cell and battery mass.

The question of how to go about improving cell power density for a DMFC has been the subject of many research studies. There are many open problems within the cell itself that, if solved, could lead to greatly improved performance including: methanol crossover, methanol oxidation kinetics, oxygen reduction kinetics, two-phase flow in the anode, cathode flooding, among others. In addition to cell-level improvements, improvements to the balance of plant such as effective air-distribution, water handling, and fuel mixing can help meet the power requirements for use in portable power applications. 
This thesis is focused on improving the DMFC by improving the performance of the anode. Electrochemical reactions are heterogeneous reactions, meaning the reaction occurs only at a catalyst surface, not in a bulk mixture. The purpose of the anode in a DMFC is to provide the sufficient catalyst area to allow the methanol oxidation reaction to proceed quickly, without requiring a large overpotenial to drive the reaction. The task is made difficult by the fact that the methanol oxidation reaction is a multi-step reaction, the nature of which is not completely understood [7]. The methanol reaction is thus inherently sluggish, meaning that large catalyst area is required to meet power demands. Catalyst area is maximized by using catalyst nanoparticles to get more area from less catalyst mass. Catalyst area, however, is not the only requirement for the reaction to proceed. The catalyst must also have an electronic connection to the external circuit, to allow electrons produced in the methanol oxidation reaction to exit the cell. The catalyst must also have an ionic connection to the cell electrolyte, so that hydrogen ions can reach the cathode side of the cell. The catalyst must also be accessible to the methanol and water fuel supply. This region of the catalyst surface where electronic, ionic, and fuel phases are all accessible is called the threephase boundary and is the region of the catalyst that can actually be used for the reaction. It is the area of this region that must be maximized in an effective DMFC anode.

The performance of the DMFC anode, is thus determined by the relative rates of many physical processes within the electrode: electronic and ionic conduction, mass transport, oxidation reaction, etc. Many of these processes in the DMFC differ significantly from their analogous process in a hydrogen-fueled anode or oxygen cathode, yet the dominant electrode used in the DMFC anode is basically the same as those used in the PEMFC with only modest changes in electrode composition to find an optimum behavior. The DMFC requirements, however, are sufficiently unique that satisfying them requires a reassessment of the electrode structure, rather than just an incremental adjustment of composition. In effect, this means treating the DMFC anode as a separate design problem which must be considered for it's specific requirements if $\mathrm{DMFC}$ anode performance is to improve.

This thesis deals primarily with two apparent limitations imposed on conventional anode designs used in DMFCs. The first is that even though additional catalyst area generally speeds up slow reactions like the methanol oxidation reaction, adding catalyst to a DMFC anode does not improve performance indefinitely. That is, there is a maximum practical catalyst loading, above which the cell performance drops with additional catalyst. The sec- 
ond limitation considered is that the use of ionomer distributed in the electrode is necessary to improve ionic conduction, yet too much ionomer can block catalyst particles and hinder performance. Several published studies of these limitations are presented in Chapter 2 that deal with DMFCs and the identical problems in hydrogen fueled PEMFCs.

In Chapter 3, the conventionally accepted design for DMFC anodes is analyzed using Axiomatic Design theory. Based on this analysis, it is proposed that coupling between the need for ionic conduction in the electrode and the simultaneous needs for methanol and carbon dioxide transport in the electrode, leads to poor anode performance. An alternative design is proposed to eliminate the coupling between the ionic conductivity function and the methanol and carbon dioxide transport functions. The alternative design uses Nafion posts in the electrode to increase ionic conductivity without blocking methanol or carbon dioxide transport. In doing so, this design should allow for catalyst in the electrode to be utilized uniformly, whether or not it is close to the membrane.

The proposed design is modeled using both one- and two-dimensional electrical network approximations, described in Chapter 3. The one-dimensional model, a transmission line circuit, illustrates the importance of improving ionic conduction within the electrode in order to balance the reaction rate within the electrode. The transmission line model also provides a sufficiently simple model with which to fit impedance data obtained from either simulation or experiment. The two-dimensional model is applied to determine the effect that post geometry has on ionic resistance in the electrode. These models are used to choose a structured electrode geometry that will reduce ionic conductivity within the electrode.

Chapter 4 describes the experimental and fabrication processes needed to evaluate the structured electrode design. Electrodes are evaluated using several test cell setups including a three-electrode cell with aqueous electrolyte, solid electrolyte cells with rigid current collectors, and solid electrolyte cells with a movable current collector. Structured electrodes for use with aqueous electrolyte are created on glassy carbon supports by casting Nafion posts from solution into aluminum molds. The mold is etched away once the casting is attached to a carbon paper backing layer to yield Nafion posts. For experiments using solid electrolyte membranes, the structured electrodes are created by embossing a Nafion membrane to form posts on the membrane surface.

Chapter 5 presents the results of cyclic voltammetry and impedance spectroscopy experiments run on both conventional and structured cells. Conventional electrodes are tested 
with varying Nafion content and with varying total catalyst loading. Structured electrodes are also tested with two structure geometries in addition to varying Nafion and catalyst loading.

Chapter 6 concludes this thesis with a summary of important results, including the key geometric parameters that control the behavior of the structured cells. The impact of the structured cells on electrode ionic resistance and catalyst available area are also discussed. While this thesis focused on the anode of the DMFC, the potential application of similar electrode structures to the DMFC and PEMFC cathode is explored. 


\section{Chapter 2}

\section{Background}

\subsection{Fuel Cell Fundamentals}

Fuel cells are electrochemical energy conversion systems. They convert the chemical energy stored in a fuel directly to electrical energy without the need for heat engine. Direct conversion from chemical to electrical energy results in increased energy conversion efficiency. Fuel cells are very similar to batteries in structure and function with the main distinction being that batteries are closed systems while fuel cells are open systems. In a fuel cell, fuel and oxidizer enters the cell and reaction products are allowed to exit the cell, while in a battery all reactants and products stay contained within the cell.

A fuel cell operates by separating a redox-couple reaction with an electrolyte. At one electrode, called the anode, the oxidation half-cell reaction occurs, while the reduction halfcell reaction occurs at the other electrode, called the cathode. The electrodes are connected externally through an electronic load and separated by an electrolyte. Electrons produced in the oxidation reaction flow through the load to reach the cathode, where they participate in the reduction reaction. Ions are produced at one electrode and flow through the electrolyte to reach the opposite electrode.

Fuel cells are typically categorized based on the type of electrolyte used. For the purposes of this thesis only PEMFCs, which use a polymer electrolyte membrane, will be considered. Details on the other common fuel cell types can be found elsewhere [3, 4]. Proton exchange membrane fuel cells are low-temperature fuel cells $\left(<100{ }^{\circ} \mathrm{C}\right)$ that use a solid polymer electrolyte to conduct hydrogen ions from the anode to the cathode. Nafion is the most well-known such electrolyte and the only one used in this thesis. There are, however, 


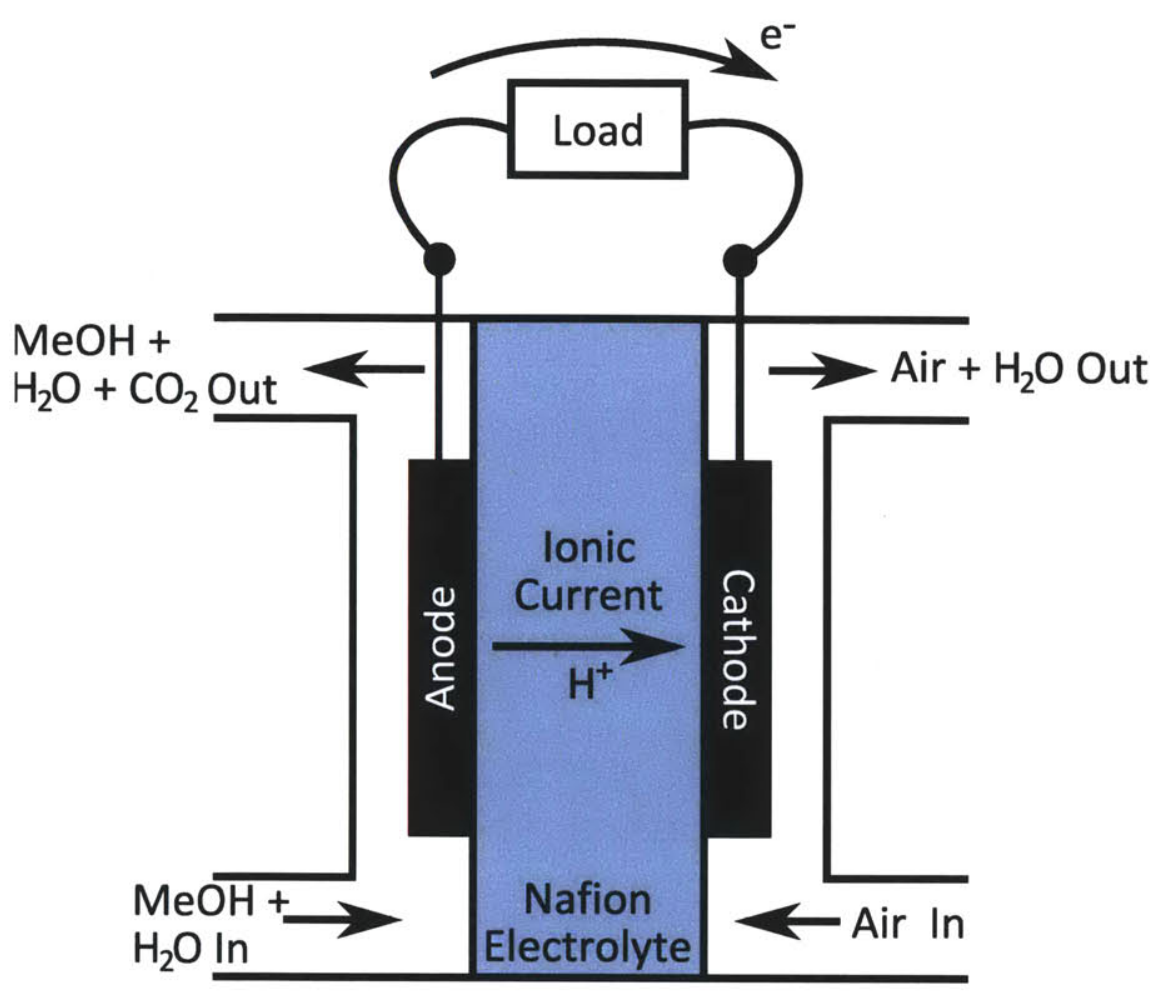

Figure 2-1: Direct methanol fuel cell schematic.

several other common electrolyte materials, and research into better materials is a very active field. Proton exchange membrane fuel cells can operate using several different fuels, the most common of which is hydrogen. Hydrogen-fuel PEMFCs have been the subject of extensive research and their performance has climbed steadily. Hydrogen, however, remains very challenging to store and reforming hydrogen from other fuels adds complexity and bulk to the PEMFC system. Methanol is another popular fuel for use in PEMFCs and cells of this type are called direct methanol fuel cells (DMFCs) so named because the methanol is oxidized directly in the cell instead of being first reformed into hydrogen. The use of methanol as fuel eliminates the fuel storage problem found in hydrogen fuel cells, but adds several other obstacles to high performance as will be shown later.

\subsubsection{Direct Methanol Fuel Cells}

A DMFC is largely similar to hydrogen-fueled PEMFC, but instead of gaseous hydrogen, liquid methanol is used as the fuel. A schematic of a DMFC is shown in Figure 2-1. Methanol and water enter the anode and the methanol is oxidized, producing carbon dioxide, electrons, and hydrogen ions. The electrons are forced out of the cell through an external load while 
the hydrogen ions are conducted through the electrolyte membrane to the cathode chamber. Electrons re-enter the cell at the cathode, then combine with oxygen gas and hydrogen ions from the membrane to reduce the oxygen to water. The overall reaction for the DMFC is:

$$
\mathrm{CH}_{3} \mathrm{OH}+\mathrm{H}_{2} \mathrm{O} \rightarrow \mathrm{CO}_{2}+3 \mathrm{H}_{2} \mathrm{O}
$$

The change in Gibbs free energy for this reaction is $-685.5 \mathrm{~kJ} / \mathrm{mol}$. The Gibbs free energy is related to the equilibrium potential of the cell as:

$$
\Delta G=-n F E
$$

where $n$ is the number of electrons transferred and $F$ is Faraday's constant. The equilibrium potential for this reaction is therefore $1.18 \mathrm{~V}$. This is the potential that would be expected between the anode and cathode if no current is flowing. Once current begins to flow, losses in the cell result in a drop in measured potential. The closer the cell potential is to the equilibrium potential, the more efficiently the cell is operating.

In electrochemical systems, reactions are heterogeneous, meaning they occur at phase interfaces rather than in the bulk mixture, as with homogeneous reactions. The overall reaction is split into two half-cell reactions, the methanol oxidation reaction at the anode catalyst surfaces

$$
\mathrm{CH}_{3} \mathrm{OH}+\mathrm{H}_{2} \mathrm{O} \rightarrow \mathrm{CO}_{2}+6 \mathrm{H}^{+}+6 e^{-}
$$

and the oxygen reduction reaction at the cathode catalyst surfaces.

$$
\frac{3}{2} \mathrm{O}_{2}+6 \mathrm{H}^{+}+6 e^{-} \rightarrow 3 \mathrm{H}_{2} \mathrm{O}
$$

In the hydrogen fueled PEMFC, the oxygen reduction reaction at the cathode is decidedly slower than the hydrogen oxidation reaction at the anode. In the DMFC, however, both reactions are relatively slow. The half-cell reactions in Equations 2.3 and 2.4 provide a guide for the requirements for a good electrode. In order for either reaction to proceed, there must be a supply of reactant available to the catalyst and the products must be able to be removed from the catalyst. A catalyst site is only considered active if it meets all these requirements. In the anode, that means the catalyst must be accessible to the methanol and water fuel 
mixture; it must be in close contact with an ionically conductive path to the membrane to remove hydrogen ions; it must provide an electronically conductive path to the current collector to remove electrons; and it must allow for the removal of carbon dioxide. Similarly, the cathode catalyst must be accessible to the oxygen supply; it must be in close contact with an ionically conductive path from the membrane to supply hydrogen ions; it must provide an electronically conductive path from the current collector to supply electrons; and it must allow for the removal of water from the catalyst. The region where these requirements are met is called the triple-phase boundary, because it is where the electronically conductive, ionically conductive, and void phases meet. Electrodes must maximize this area in order to perform well in a fuel cell.

\subsubsection{Sources of Inefficiency in Fuel Cell Systems}

According to Equations 2.1 and 2.2, the potential across one cell in a DMFC should be $1.18 \mathrm{~V}$ at equilibrium. This is only true when there is no current drawn from the cell. As soon as current begins to flow, the potential of the cell drops. The ratio of the measured potential to the open circuit potential is the efficiency of the cell referenced to the maximum energy output of the cell, the Gibbs free energy of the reaction.

The inefficiency of fuel cells are generally divided into three categories: activation losses, ohmic losses, and mass-transfer losses. Activation losses are a result of reaction kinetics at the anode and the cathode catalysts. In order to drive the reactions at the catalyst surface, a slight bias potential, called an overpotential, must be applied. If the reaction is inherently fast, like the hydrogen oxidation reaction, then the activation overpotential is small, but

still non-zero. If, however, the reaction is sluggish, like the methanol oxidation reaction, a larger overpotential is required, thus reducing efficiency. A given overpotential will drive a specific current density at the electrode surface, so activation losses can be reduced by adding catalyst area to increase current without additional overpotential. Ohmic losses are simply resistance to conduction throughout the cell. The major contributors to ohmic losses are ionic resistance in the electrolyte and contact resistances between cell components. Mass transfer losses are the final category of inefficiencies found in fuel cells. The mass-transfer overpotential in a fuel cell is a result of the reactant being consumed at the catalyst faster than it can be replenished. As the reaction rate increases, the concentration of the reactant at the catalyst must drop. Since the equilibrium potential is determined by the concentration 


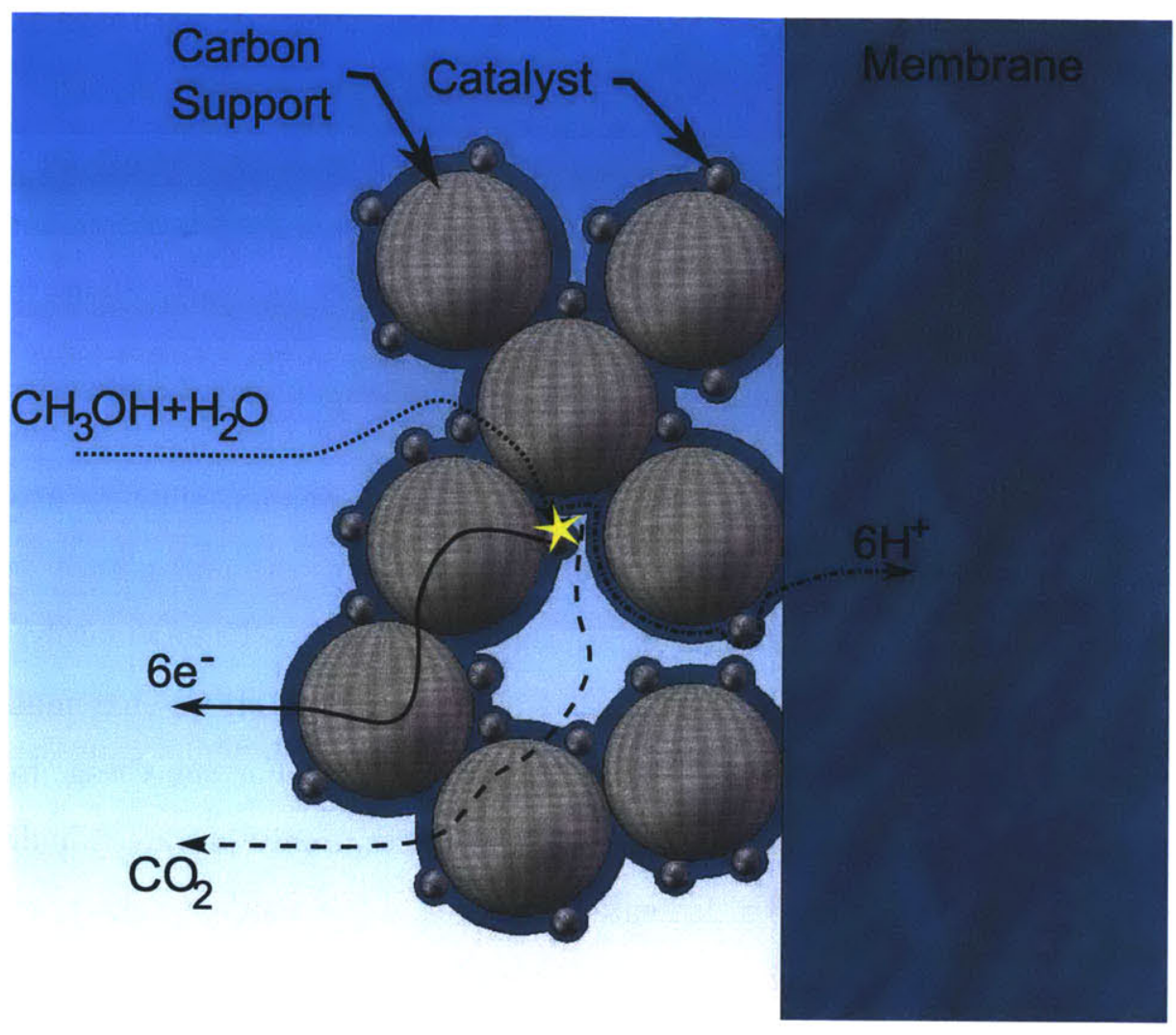

Figure 2-2: Schematic representation of a portion of a typical DMFC anode structure.

of reactants and products at the surface through the Nernst Equation [8], the potential must drop as the reactant concentration drops. This potential drop is the mass-transfer overpotential and it becomes more significant at higher currents. In fact, the maximum current in a fuel cell is limited by mass-transfer: once the concentration of the reactants at the catalyst surface reaches zero, the reaction has reached its limiting current and can go no faster.

In general, activation losses dominate fuel cell behavior at low currents, ohmic losses become significant in the middle range of currents, and mass-transfer losses dominate high-current behavior, though all three are present throughout cell operation.

\subsubsection{Electrode Structure}

The most common electrode structure used in PEMFCs today is carbon-supported catalyst. In this arrangement, as shown in Figure 2-2, catalyst particles on the order of $3 \mathrm{~nm}$ in diameter are deposited onto carbon black particles with diameters on the order of $30 \mathrm{~nm}$. This catalyst powder is combined with an ionomer to bind the particles and conduct ions 
through the electrode. The resulting electrode is a porous structure with carbon particles allowing electronic conduction throughout the electrode. The catalyst particles are small enough to provide large surface area per unit mass, and the binding ionomer provides ionic access to the entire electrode.

This structure has been the basis of most PEMFCs since the early 1990s [9]. It was adapted from Phosphoric Acid Fuel Cells (PAFCs) for use in hydrogen fueled PEMFCs, and its dominance in that field made it a natural favorite for DMFCs. The main change is that the anode catalyst used in DMFCs is a platinum-ruthenium alloy, to avoid carbon monoxide poisoning, rather than just platinum for hydrogen. At first glance, translating this structure to DMFCs seems to be an appropriate decision. Unfortunately, there are several key differences between hydrogen-fueled PEMFCs and DMFCs that make copying the anode structure more problematic than it initially seems. For one thing, hydrogen is a gaseous fuel and the hydrogen oxidation half-cell reaction only produces hydrogen ions and electrons, both of which have a dedicated path out of the anode, so the pores are only used to distribute hydrogen. Methanol, on the other hand, is a liquid fuel and the methanol oxidation reaction produces $\mathrm{CO}_{2}$ in addition to hydrogen ions and electrons, so the pores in the anode must allow methanol to reach the catalyst sites and allow $\mathrm{CO}_{2}$ to leave the catalyst sites. The problems associated with this two-phase flow have been considered in several studies. Another major difference between the two fuel options is that the methanol oxidation reaction is much slower than the hydrogen oxidation reaction. This means that significantly more catalyst is required to deliver sufficient power density.

\subsection{History of porous fuel cell electrodes}

\subsubsection{Early porous electrodes}

Early PEMFCs used porous electrodes adapted from fuel cells with liquid electrolytes, such as PAFCs. These electrodes contained carbon supported catalyst but no ionomer content to conduct hydrogen ions. The performance of these electrodes was maximized by concentrating catalyst near the membrane. Typical strategies involved using large amounts of unsupported catalyst particles and hot pressing the particles into the membrane. High pressure attachment meant deeper ionomeric penetration, better ion conduction, more active area and therefore better performance. 


\subsubsection{Ionomer Impregnated Electrodes}

Electrodes formed by pressing unsupported catalyst into the membrane turned out to use far too much catalyst material. In 1986 Raistrick [10] proposed a method to impregnate carbon-supported catalyst electrodes from PAFCs with a layer of Nafion to replicate the behavior found in PAFCs when the liquid electrolyte can penetrate the electrode thus providing ionic continuity deeper into the electrode. His method involved painting a preformed electrode with Nafion solution and allowing it to dry before hot-pressing the electrode onto the membrane. This design change increased ionic conductivity and allowed catalyst to be distributed more evenly throughout the electrode, rather than simply compressed into the membrane. Current density was improved by an order of magnitude by adding the layer of Nafion.

Ticianelli et al [11] used this method and found that the ideal Nafion loading in the electrode was only $3.3 \%$. Beyond this level, electrode performance began to suffer. This is the level at which the painted-on Nafion solution would begin to block the electrode pores.

\subsubsection{Ionomer Based Inks}

In 1992, Wilson and Gottesfeld [9] further improved upon electrode performance and ushered in the current standard in PEMFC electrode structure, when they mixed Nafion solution directly into the ink used to create the catalyst layer. This method allows the ionomer to be distributed evenly throughout the electrode thickness and thus avoid blocking the pores near the membrane if too much Nafion is applied. In fact, the cells they fabricated used $25 \%$ Nafion by mass, up from the $3.3 \%$ limit in Nafion impregnated electrodes.

\subsection{Ionic Resistance in Fuel Cell Electrodes}

The desire to improve ionic conductivity in fuel cell electrodes motivated much of the evolution of porous electrodes to their current design. Not surprisingly, there is a significant body of research that deals with ionic resistance in electrodes and mitigating its deleterious effects.

Springer et al [12] were early adopters of electrochemical impedance spectroscopy to study fuel cell behavior and use it to parse the different sources of impedance from single experiments. Unlike the studies in this thesis, their experiments used full cells under 
operating conditions, as opposed to the blocking electrodes described in Chapter 4. They found that ionic resistance in the electrode manifests itself in a Nyquist plot as a $-45^{\circ}$ line in the high-frequency regime. This behavior is identical to that predicted and observed using blocking electrodes in this thesis, except that with blocking electrodes, the Nyquist plot approaches infinite imaginary impedance at low frequencies. In cells tested in operating conditions, however, the Nyquist plot returns to the real axis, thanks to the faradaic reaction. They showed that decreasing ionic resistance in the electrode will improve performance, particularly in the mid-range current densities, but the cell's limiting current is unchanged by decreases in ionic resistance.

\subsubsection{Optimum Nafion Loading}

One of the common topics of interest developing from the study of ionic conductivity in fuel cell electrodes is the determination of the ideal Nafion content for an electrode. As described in Section 2.2, early electrodes used no Nafion in the electrode and performance suffered. In electrodes impregnated with Nafion, the layer closest to the membrane had far too much Nafion, while the rest of the electrode had far too little, thus limiting performance gains. The current standard in porous electrodes uses well-distributed Nafion and has, to date, offered the best performance, but what is the ideal Nafion content?

Li and Pickup [13] reported on a study of ionic conductivity in PEMFC electrodes using a combination impedance spectroscopy on blocking cells and full cell polarization curves. They ran their impedance cell as a blocking electrode, under nitrogen, and modeled the data using a transmission line model with ionic resistance that varies with position. They found that the best performing electrodes, based on polarization curves, have a Nafion loading of approximately $30 \%$ by mass. The polarization data was subjected to Tafel analysis [14] to fit the polarization curve as:

$$
E=E_{o}-b \log i-R i
$$

They removed the high-frequency intercept for each cell, found using impedance spectroscopy, from the resistance term, $R$, in Equation 2.5. Plotting this remaining resistance as a function of Nafion loading results in a minimum value at the best performing cell with $30 \%$ mass Nafion. 


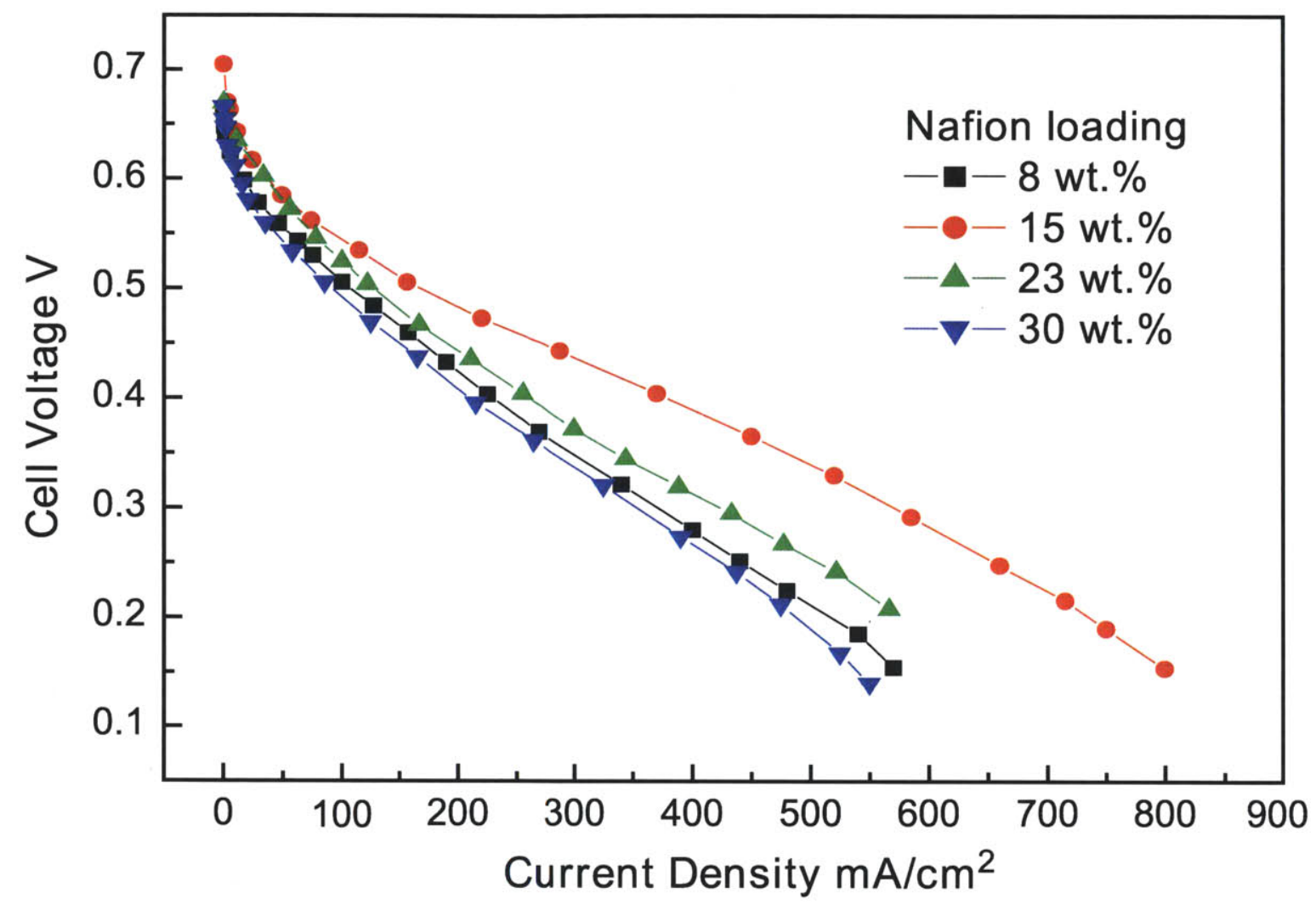

Figure 2-3: Polarization curves for DMFCs with several anode Nafion loadings. Taken from Zhao et al [1].

Passalacqua et al [15] performed a study of porous PEMFC electrodes aiming to find the optimum Nafion loading in an electrode. Operating their hydrogen/air fuel cells, they found that the ideal Nafion loading to be $33 \%$ by mass in the electrode. They also found that the optimum Nafion loading does not change with catalyst loading in the electrode. In addition, they used mercury intrusion poisometry to show that too much Nafion in the electrode does, in fact, block the electrode pores and proposed that this is the reason that too much Nafion will decrease cell performance.

Krishnamurthy et al [16] studied DMFC anodes and cathodes as a function of Nafion content. They found that $25 \%$ Nafion loading was the optimum content for DMFC electrodes. Zhao et al [1] also studied the effects of Nafion loading on DMFC performance. The polarization plots they measured are shown in Figure 2-3, and indicate that the optimum Nafion loading is $15 \%$ by weight. They used Pt-Ru black, rather than carbon supported catalyst in their anode, which is likely the reason that their optimum Nafion loading is lower than that of the other groups. Since there is only catalyst in the electrode, every 
bit of Nafion within the Pt-Ru electrode is blocking catalyst, whereas only a portion of the Nafion in the carbon supported electrode blocks catalyst. This makes the deleterious effects of too much Nafion appear only after more Nafion has been added. They also report that electronic resistance in the electrode increases sharply at Nafion loadings greater than approximately $20 \%$ by mass. This suggests that increased electronic resistance, in addition to pore blocking, could hinder cell performance as Nafion content increases.

\subsubsection{Catalyst Loading Limit}

One potential consequence of high ionic resistance is the emergence of a catalyst loading limit in fuel cell electrodes. In general, more catalyst is associated with better electrode performance. Additional catalyst, however, also leads to thicker electrodes and therefore larger total ionic resistance.

Shimizu et al [2] built a passive air-fed DMFC with several levels of anode catalyst loading. Figure 2-4 shows the polarization plot and power curve for these cells. They found that catalyst loading of $2.5 \mathrm{mg} / \mathrm{cm}^{2}$ would maximize performance over all current densities, but higher or lower loading would decrease performance.

Bae et al [17] also reported on observing an optimum catalyst loading in passive DMFCs. They found that the best performance was at a catalyst loading of $6 \mathrm{mg} / \mathrm{cm}^{2}$, though they did not report a steep performance decline as Shimizu et al did, but rather a plateau. They attribute the lack of decline to their use of Pt-Ru black, as opposed to carbon supported catalyst. The use of Pt-Ru black means that the thickness of the electrode increases more slowly with additional catalyst, reducing the effect of conduction or other transport losses.

\subsection{Previous Structured Electrodes}

The electrode design proposed herein aims to reduce ionic resistance in the electrode by embossing Nafion posts onto the membrane before applying the electrode ink. Despite this method's simplicity, there have been few similar attempts published in the literature.

Yildririm et al [18] used a similar embossing method with the stated aim of improving DMFC performance by increasing electrode to membrane contact area. They textured a Nafion membrane by embossing fairly low aspect ratio features onto the surface before testing full DMFCs. Unfortunately, the texture applied to the membrane was almost completely 


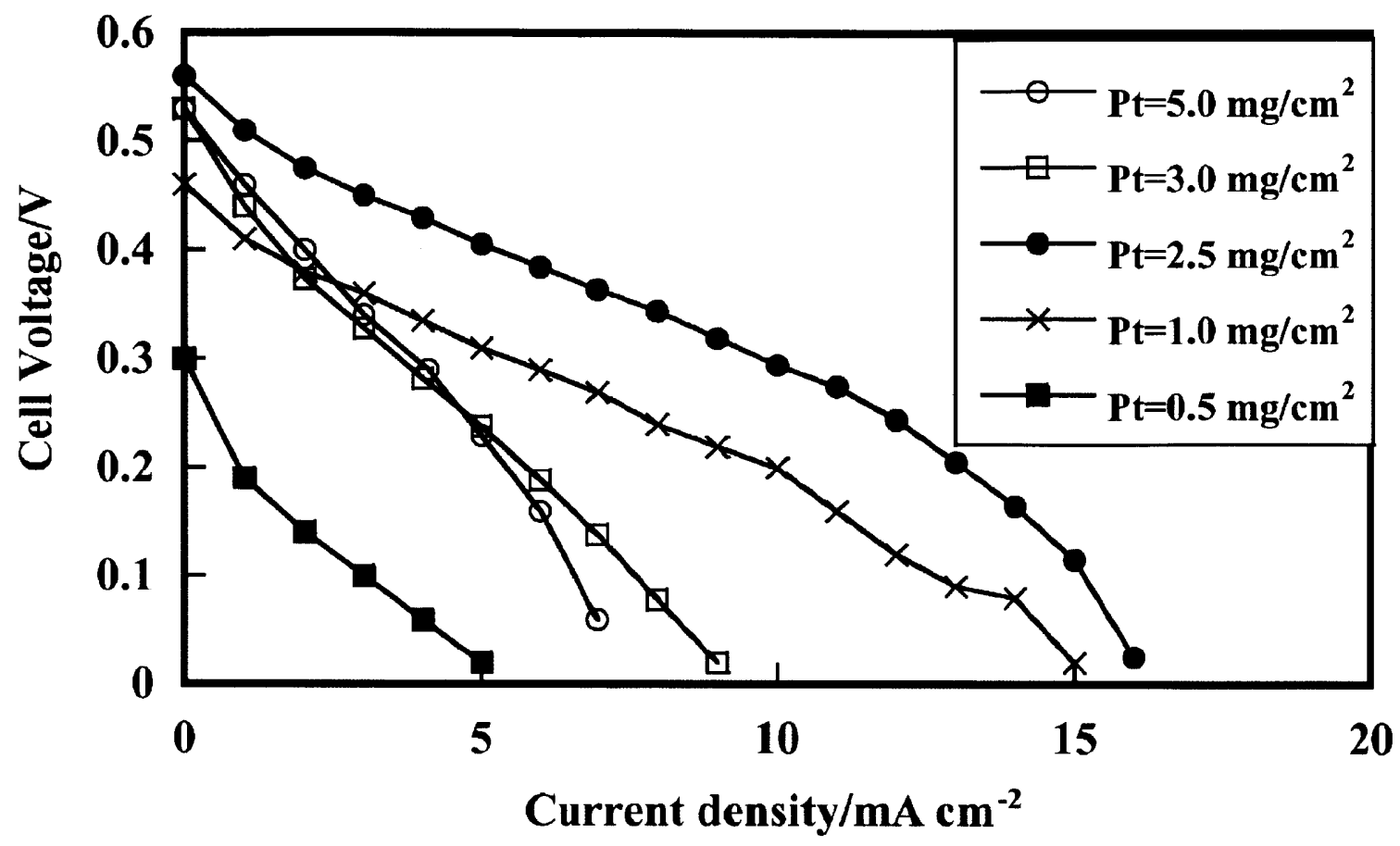

(a)

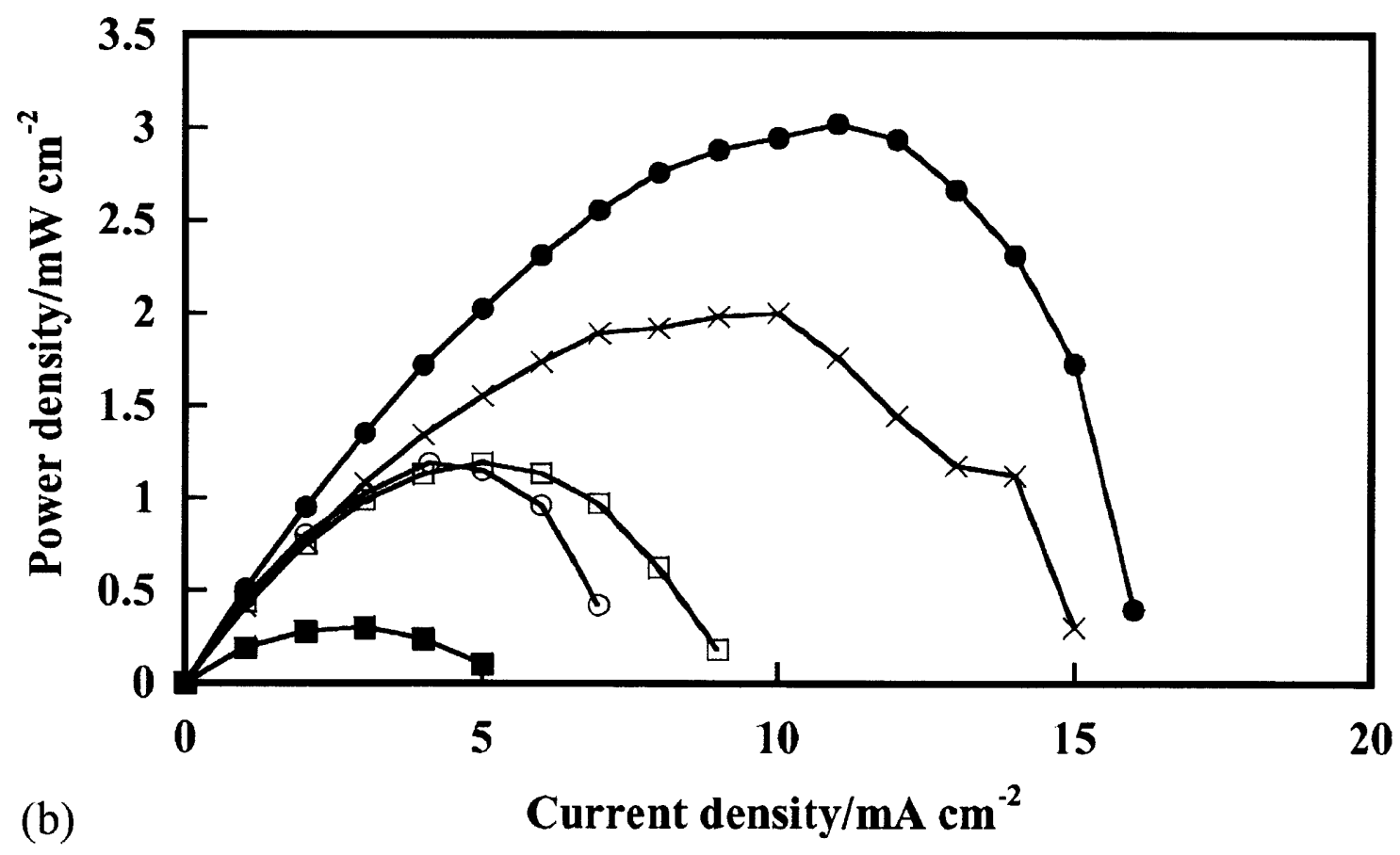

(b)

Figure 2-4: Polarization (a) and power density (b) plots for different catalyst loadings. Taken from Shimizu et al [2] 
removed by the application of the electrode to the textured membrane and the textured cells performed no better than cells that had no texture applied. The fabrication method presented in Chapter 4 avoids this issue by hot-pressing the counter electrode before the posts have been removed from the mold.

Zhang et al $[19,20]$ have also used embossing to improve DMFC performance. The cells they fabricated, however, had little resemblance to the cells of interest in this thesis. They were working on micro DMFCs that use a sputtered layer of platinum as the anode, rather than the conventional porous carbon supported catalyst. Since there is no ionomer in the electrode, only the area between the platinum and the membrane can be active. The texture is applied to increase effective catalyst surface area, rather than to improve ionic resistance. The texture turns out to have the added benefit of forming more cracks in the sputtered platinum to improve fuel distribution in addition to active area.

Zhou et al [21] also created fuel cells using textured membranes, however, their main focus was the method used to produce the membranes, rather than the impact the texture itself has on performance. They created a liquid precursor from which the membrane was cast onto several different molds. Their cells use pits in the membrane rather than posts extending into the electrode, but the effect should be similar. They observed decidedly better performance in full cell tests using hydrogen fuel. However, they compared their cells made with a unique membrane material to a cell made with flat Nafion so it is unclear how much of this performance gain relates to the texture and how much relates to the membrane material. Their production method is intriguing, but effect of the texture on the performance of their cells is not yet well characterized as it relates to ionic conduction in the electrode. 


\section{Chapter 3}

\section{Design and Analysis of Structured Electrodes}

\subsection{Axiomatic Design Analysis of Porous Electrodes}

\subsubsection{Axiomatic Design Theory}

Axiomatic Design is a useful tool for analysis of challenging design problems. What follows is a brief overview of some of the crucial points of Axiomatic Design theory [22, 23].

In its simplest form, Axiomatic Design involves the selection of Design Parameters (DPs) to meet a set of Functional Requirements (FRs). FRs are the minimum set of requirements that the design must satisfy to be considered a success. DPs are physical systems, features, or variables that are selected to meet the needs defined by the FRs. The process of mapping what we want to achieve-defined in the functional domain by FRs-to how we will achieve it-defined in physical domain by the DPs-is the Axiomatic Design process. The quality of the design is evaluated based on two axioms: the independence axiom and the information axiom. The independence axiom states that all FRs should be satisfied independently. The information axiom states that information content of the design should be minimized, where information content is related to the probability of failing to meet a given FR. In general, the independence axiom is used to determine if a set of DPs is a 'good' set, while the information axiom is used to evaluate how robust the design is to variations in the DPs. For the purposes of this thesis, only the independence axiom will be considered.

DPs are often thought of as the levers or variables that the design can adjust to satisfy 
FRs. An ideal design must have the same number of DPs available as there are FRs to satisfy. If there are too few DPs, then some DPs must be used to satisfy several FRs, meaning that those FRs are no longer independent from one another. In order for FRs to be considered independent and satisfy the first axiom, the DPs associated with those FRs must have minimal effect on the satisfaction of the other FRs. The interactions between the DPs and the FRs can be formulated as:

$$
\{F R\}=[A]\{D P\}
$$

where the FRs and their associated DPs are arranged into column vectors $\{F R\}$ and $\{D P\}$, respectively. The matrix, $A$, is called the design matrix and it contains terms that map the influence of DPs onto the set of FRs. The elements in the design matrix can be expressed as:

$$
A_{i j}=\frac{\partial F R_{i}}{\partial D P_{j}}
$$

It is often the case that the precise relationship between FRs and DPs is not available or known, but the matrix structure can still be ascertained. In such cases, the terms in the design matrix, $A_{i j}$, are replaced with an $X$, if there is an interaction, or with a 0 , if there is no interaction. The degree of coupling can still be determined using this simplified representation.

The design matrix then becomes a very powerful tool for evaluating how well a given set of DPs satisfies the independence axiom. In order for all the FRs to be independent from each other, the design matrix must be a diagonal matrix or a triangular matrix. In the diagonal case, each DP effects only one FR. The DPs can be chosen in any order and the design should function as expected provided that each DP is capable of meeting its associated FR. In the case with a triangular matrix, the FRs can still be independently satisfied, but only if the DPs are chosen in the correct order.

To illustrate this situation, consider the case of a $3 \times 3$ lower-triangular design matrix, shown in Table 3.1b. In this case, $D P_{1}$ has an effect on all three FRs, so it must be set first to meet $F R_{1}$. Once $D P_{1}$ is chosen, then $D P_{2}$, which effects $F R_{2}$ and $F R_{3}$, can be used to satisfy $F R_{2}$ without effecting the already satisfied $F R_{1}$. Finally, $D P_{3}$ is used to satisfy $F R_{3}$ and has no effect on the first two FRs, so the design goals have now been met. If the 
Table 3.1: Examples of (a) uncoupled (b) decoupled and (c) coupled design matrices.

(a) Uncoupled design matrix

\begin{tabular}{|c||c|c|c|}
\cline { 2 - 4 } \multicolumn{1}{c|}{} & $D P_{1}$ & $D P_{2}$ & $D P_{3}$ \\
\hline \hline$F R_{1}$ & $\mathrm{X}$ & 0 & 0 \\
\hline$F R_{2}$ & 0 & $\mathrm{X}$ & 0 \\
\hline$F R_{3}$ & 0 & 0 & $\mathrm{X}$ \\
\hline
\end{tabular}

(b) Decoupled design matrix

\begin{tabular}{|c||c|c|c|}
\cline { 2 - 4 } \multicolumn{1}{c||}{} & $D P_{1}$ & $D P_{2}$ & $D P_{3}$ \\
\hline \hline$F R_{1}$ & $\mathrm{X}$ & 0 & 0 \\
\hline$F R_{2}$ & $\mathrm{X}$ & $\mathrm{X}$ & 0 \\
\hline$F R_{3}$ & $\mathrm{X}$ & $\mathrm{X}$ & $\mathrm{X}$ \\
\hline
\end{tabular}

(c) Coupled design matrix

\begin{tabular}{|c||c|c|c|}
\cline { 2 - 4 } \multicolumn{1}{c|}{} & $D P_{1}$ & $D P_{2}$ & $D P_{3}$ \\
\hline \hline$F R_{1}$ & $\mathrm{X}$ & $\mathrm{X}$ & $\mathrm{X}$ \\
\hline$F R_{2}$ & $\mathrm{X}$ & $\mathrm{X}$ & $\mathrm{X}$ \\
\hline$F R_{3}$ & $\mathrm{X}$ & $\mathrm{X}$ & $\mathrm{X}$ \\
\hline
\end{tabular}

DPs were chosen out of this order, then the FRs could not be independently set, and the first axiom would be violated. In the case of a full matrix, every DP effects every FR and there is no order which will satisfy the independence axiom. These three cases are named based on their ability to satisfy the independence axiom. The diagonal matrix case is called an uncoupled design, the triangular matrix case is called a decoupled design, while the full matrix case is called a coupled design. The uncoupled design is the ideal case, where the FRs are completely independent. The decoupled case is acceptable, but the off-diagonal terms make the design less robust and therefore it often can run afoul of the information axiom. The coupled case is very problematic and should be avoided by proper choice of DPs. In the coupled case, a satisfactory solution can sometimes be found by optimization, but the result is generally not a robust design.

\subsubsection{Conventional Porous Electrode Decomposition}

Axiomatic Design analysis of DMFC anodes provides interesting insight into the nature of the electrode issues. In this analysis, only the anode is considered and other standard components of the fuel cell are assumed to be constraints.

The top level function of the DMFC anode is to facilitate the methanol oxidation reaction. This can be broken down into five FRs for the anode. First, the anode must provide catalyst sites for the methanol oxidation reaction. The anode must also allow methanol and water to reach the catalyst from the fuel supply. It must also allow for the removal of all the products of the methanol oxidation reaction. This includes the removal of carbon dioxide from the anode, the conduction of electrons from the catalyst to the current collector, and the conduction of hydrogen ions from the catalyst to the Nafion membrane.

In the current electrode design, described in Chapter 2, these five FRs are satisfied by an equal number of DPs. The catalyst sites for the methanol oxidation reaction are provided by the use of platinum-ruthenium nanoparticles in the electrode. The electrode's porous 
structure allows for the methanol fuel mixture to permeate the anode and reach the catalyst particles. Typical DMFC anodes are backed by a hydrophilic gas diffusion layer, which has been shown to improve removal of carbon dioxide from the anode. The anode is built on a support of carbon-black nanoparticles, which form an electronically conductive structure that will conduct electrons from the catalyst to the current collector. The final task of conducting hydrogen ions from the catalyst to the membrane is achieved with a dispersed layer of Nafion throughout the electrode.

The complete list of five FRs is then:

$F R_{1}$ : Provide catalyst sites for the methanol oxidation reaction

$F R_{2}$ : Allow methanol fuel mixture to reach catalyst sites in the anode

$F R_{3}$ : Allow carbon dioxide to exit the anode from the catalyst sites

$\mathrm{FR}_{4}$ : Conduct electrons from the catalyst sites to the current collector

$F R_{5}$ : Conduct hydrogen ions from the catalyst sites to the membrane

And those functions are satisfied with the following DPs:

$D P_{1}: \quad$ Platinum-Ruthenium catalyst nanoparticle loading

$D P_{2}$ : Porous electrode structure

$D P_{3}$ : Hydrophilic gas diffusion layer

$D_{4}$ : Conductive carbon nanoparticle supports

$D P_{5}$ : Dispersed Nafion layer throughout the electrode

The design matrix for the conventional DMFC anode is shown in both in Tables $3.2 \mathrm{~b}$ and 3.2a. To assemble this design matrix, the influences of each DP must be evaluated. The loading of catalyst particles included in the anode, $D P_{1}$, has a strong influence on the electrode thickness. The more catalyst is required in the anode, the thicker the anode must become. The additional anode thickness has a deleterious effect on the transport processes in the electrode which include methanol transport, $F R_{2}$; carbon-dioxide transport, $F R_{3}$; and hydrogen ion conduction, $F R_{5}$. It would also increase electronic resistance, however, the electronic conductivity in the electrode is so high that this effect is negligible. The porous electrode structure, $D P_{2}$, needed to ensure good methanol transport, results in the ion conductive Nafion paths through the electrode being very convoluted and thus increasing ionic resistance, $F R_{5}$. The layer of Nafion spread through the electrode, $D P_{5}$, if applied in excess can block methanol access to the catalyst particles, $F R_{2}$, or carbon-dioxide evolution 
Table 3.2: Design matrix for a conventional porous DMFC anode.

(a) Unsorted design matrix

(b) Sorted design matrix

\begin{tabular}{c||c|c|c|c|c|c||c|c|c|c|c|} 
& $D P_{1}$ & $D P_{2}$ & $D P_{3}$ & $D P_{4}$ & $D P_{5}$ & & $D P_{1}$ & $D P_{2}$ & $D P_{5}$ & $D P_{4}$ & $D P_{3}$ \\
\hline \hline$F R_{1}$ & $\mathrm{X}$ & & & & $\mathrm{X}$ \\
\hline$F R_{2}$ & $\mathrm{X}$ & $\mathrm{X}$ & & & $\mathrm{X}$ \\
\hline$F R_{3}$ & $\mathrm{X}$ & & $\mathrm{X}$ & & $\mathrm{X}$ \\
\hline$F R_{4}$ & & & & $\mathrm{X}$ & \\
\hline$F R_{5}$ & $\mathrm{X}$ & $\mathrm{X}$ & & & $\mathrm{X}$ & $\mathrm{X}$ & & $\mathrm{X}$ & & \\
\hline$F R_{2}$ & $\mathrm{X}$ & $\mathrm{X}$ & $\mathrm{X}$ & & \\
\hline$F R_{5}$ & $\mathrm{X}$ & $\mathrm{X}$ & $\mathrm{X}$ & & \\
\hline$F R_{4}$ & & & & $\mathrm{X}$ & \\
\hline$F R_{3}$ & $\mathrm{X}$ & & $\mathrm{X}$ & & $\mathrm{X}$ \\
\hline
\end{tabular}

from the catalyst particles, $F R_{3}$. The results of this analysis are shown on the raw design matrix in Table 3.2a and in the design matrix which has been sorted to highlight the source of coupling in this design, as shown in Table 3.2b. From the design matrix, it is clear that changing $D P_{5}$ to eliminate its influence on methanol transport, $F R_{2}$, will alleviate the coupling in this system. It is also clear from the design matrix why the typical design strategy for a fuel cell electrode is to make it as thin as possible. The amount of catalyst needed in the electrode, $D P_{1}$, and therefore the electrode's thickness, has an influence on most other anode functions, which make the design more sensitive, even if it is a decoupled system.

\subsection{Axiomatic Design Inspired Solution}

It is clear from the historical development of fuel cell electrodes $[9,11,10]$, from the studies of current electrode designs $[16,24]$ and from the axiomatic design analysis that ionic conduction within the DMFC anode is a major performance limiter. The axiomatic design analysis makes it clear that there must be a higher-level design change in order to improve performance of the DMFC anode by modifying ionic conductivity. Simply optimizing the current electrode structure will not be sufficient.

One potential solution is to add ionically conductive channels through the thickness of the electrode to satisfy the ionic conductivity function of the electrode, thus replacing $D P_{5}$. This one change has significant impact on the anode's design matrix. The ionically conductive channels will have geometry that is independent of the electrode porosity, so $D P_{2}$ will no longer influence ionic conductivity within the electrode. Similarly, the ionic conduction channels will have large cross-sections, compared with the previous dispersed Nafion layers. Thus, the ionic resistance will not grow as quickly as more catalyst is added 
Table 3.3: Design matrix for the proposed structured anode design with ionic conductive channels through the electrode thickness.

\begin{tabular}{c||c|c|c|c|c|} 
& $D P_{1}$ & $D P_{2}$ & $D P_{5}$ & $D P_{4}$ & $D P_{3}$ \\
\hline \hline$F R_{1}$ & $\mathrm{X}$ & & 0 & & \\
\hline$F R_{2}$ & $\mathrm{X}$ & $\mathrm{X}$ & 0 & & \\
\hline$F R_{5}$ & 0 & 0 & $\mathrm{X}$ & & \\
\hline$F R_{4}$ & & & & $\mathrm{X}$ & \\
\hline$F R_{3}$ & $\mathrm{X}$ & & 0 & & $\mathrm{X}$ \\
\hline
\end{tabular}

to the electrode, thus eliminating the term in the design matrix between $D P_{1}$ and $F R_{5}$. Perhaps more importantly, increasing the ionic conductivity of the electrode can be achieved by increasing the diameter of the ionic channels, rather than the thickness of the dispersed Nafion layer throughout the electrode. This eliminates the threat of blocking methanol access to catalyst particles, or blocking carbon-dioxide removal from the catalyst particles in search of higher ionic conductivity. Controlling ionic conductivity with the post geometry, therefore, removes the influence of $D P_{5}$ on both $F R_{2}$ and $F R_{3}$. The resulting design matrix for this design is shown in Table 3.3.

It is important to note that this proposed design does not do away with the dispersed Nafion layer in the electrode, but rather adds Nafion posts, while retaining all other electrode features. This can be thought of as having an extra DP available to satisfy the FRs. In this situation, Axiomatic Design Theory stipulates that one of the extra DPs should be frozen at a convenient setting, thus keeping the number of DPs equal to the number of FRs [23].

A two-dimensional diagram of the proposed electrode is shown in Figure 3-1. To best understand the potential benefits of this design, it is helpful to divide the space between the posts into two regions. In Region A, the electrode material will see little difference between the unstructured and the structured case, because the closest sink for hydrogen ions is still the original membrane surface. In Region $\mathrm{B}$, however, the closest sink for hydrogen ions is now the Nafion posts and the catalyst in this region should perform much better than without the texture. Since the resistance is lower in the posts than in the electrode bulk, the relative improvement should be greater farther from the membrane. From this illustration, the preferred post geometry seems to be tall posts packed closely together. This choice must be balanced by the realization that the addition of posts displaces electrode material, so the posts should neither be too close, nor too larger in diameter. 


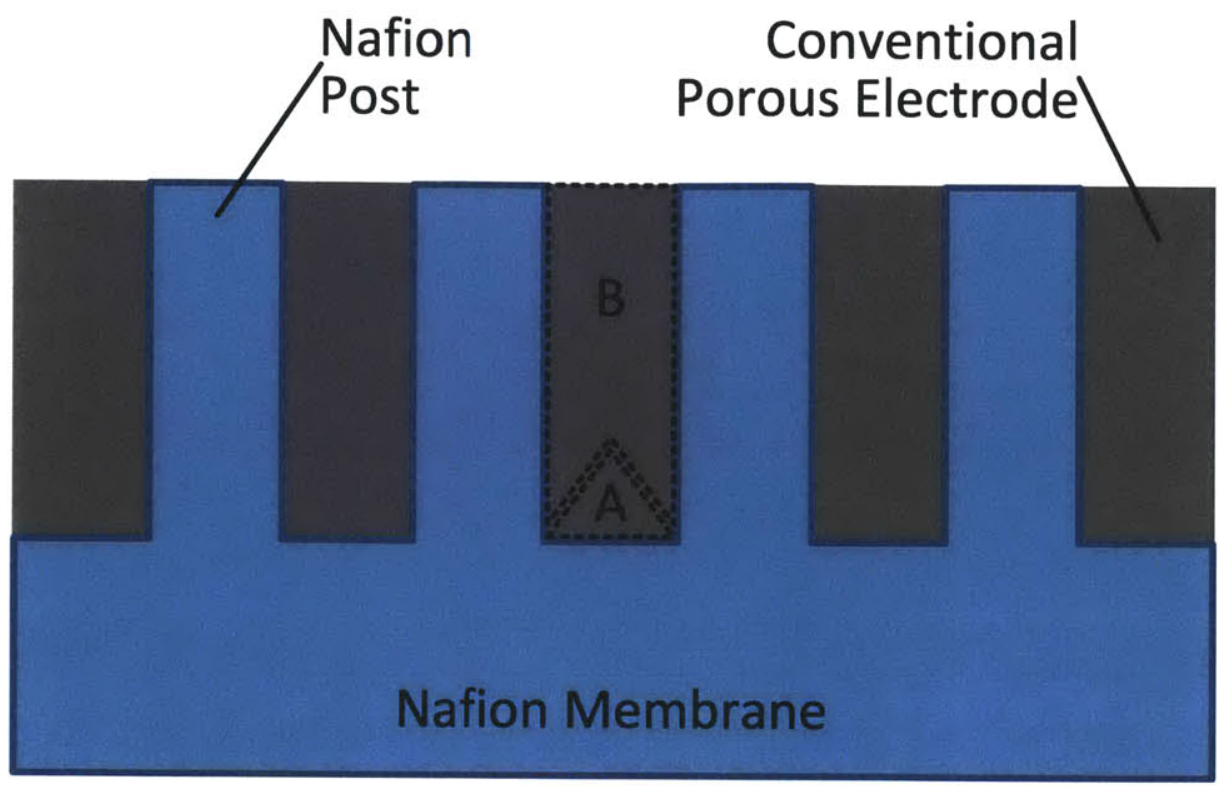

Figure 3-1: Diagram of Nafion post electrode.

\subsection{Transmission Line Model for a DMFC Anode}

Before delving into the potential performance of a structured electrode, the performance of conventional electrodes must first be considered to illustrate how the coupling terms arise in the anode design matrix in Table 3.2. An electrical network model of the electrochemical conversion process in the porous fuel cell electrode can yield significant insight into the effect that ionic resistance has on other anode FRs. This type of model is frequently used in the analysis of porous electrodes [25]. The foundation of this model is the realization that there are two conductive regimes in a porous electrode: the ionic conductive regime and the electronic conductive regime. These two regimes are connected through the faradaic process that occurs at the catalyst particles throughout the electrode. The surface overpotenial at the catalyst, which drives the faradaic conversion process, varies through the thickness of the electrode as as a consequence the rate of conversion from one regime to the other also varies with thickness. In the case of the anode, the full cell current enters in the electronic conduction regime at the back of the electrode from the current collector and no ionic current can flow in or out at this side of the electrode. As current flows through the electrode towards the membrane, it is converted from the electronic regime to the ionic regime. At the membrane, all electronic current that entered the electrode has been converted to ionic current and exits to the membrane. 


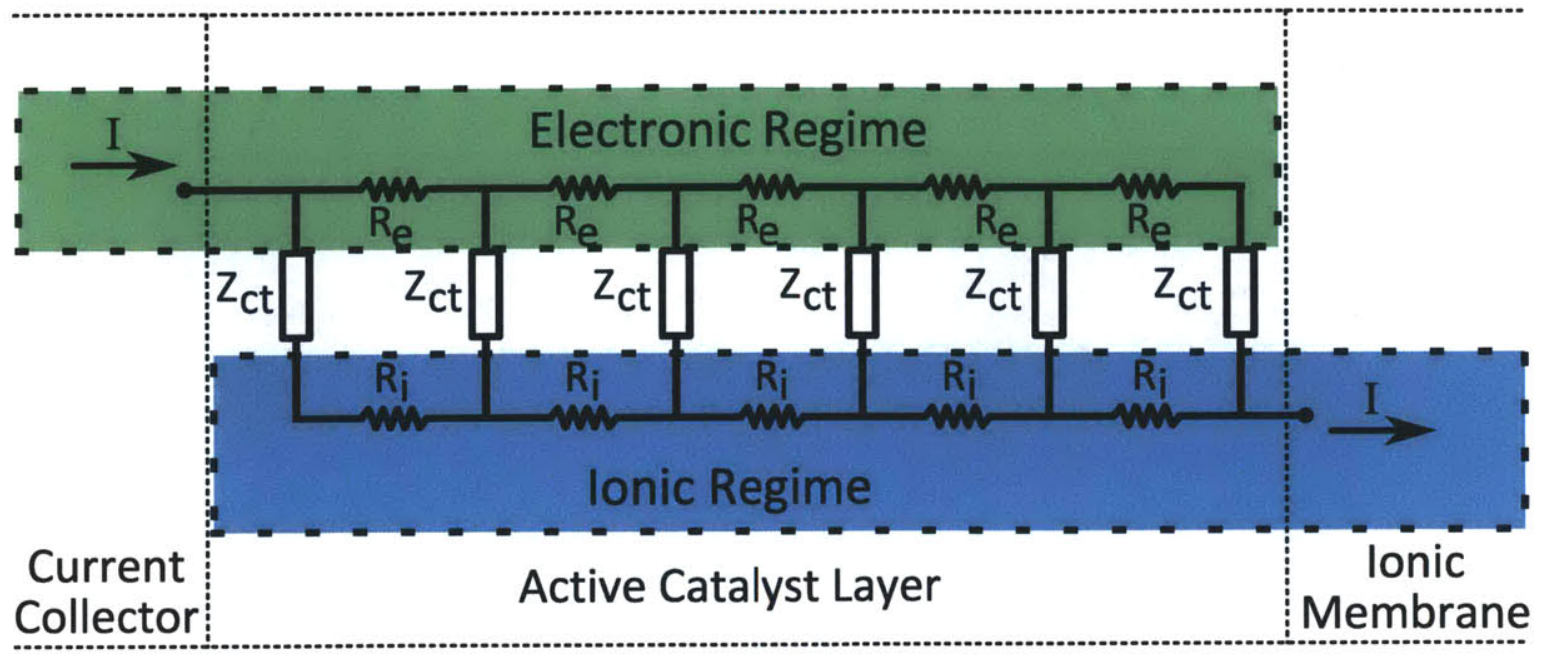

Figure 3-2: Diagram of the network electrode model showing the two separate conduction regimes connected through a non-linear faradaic process.

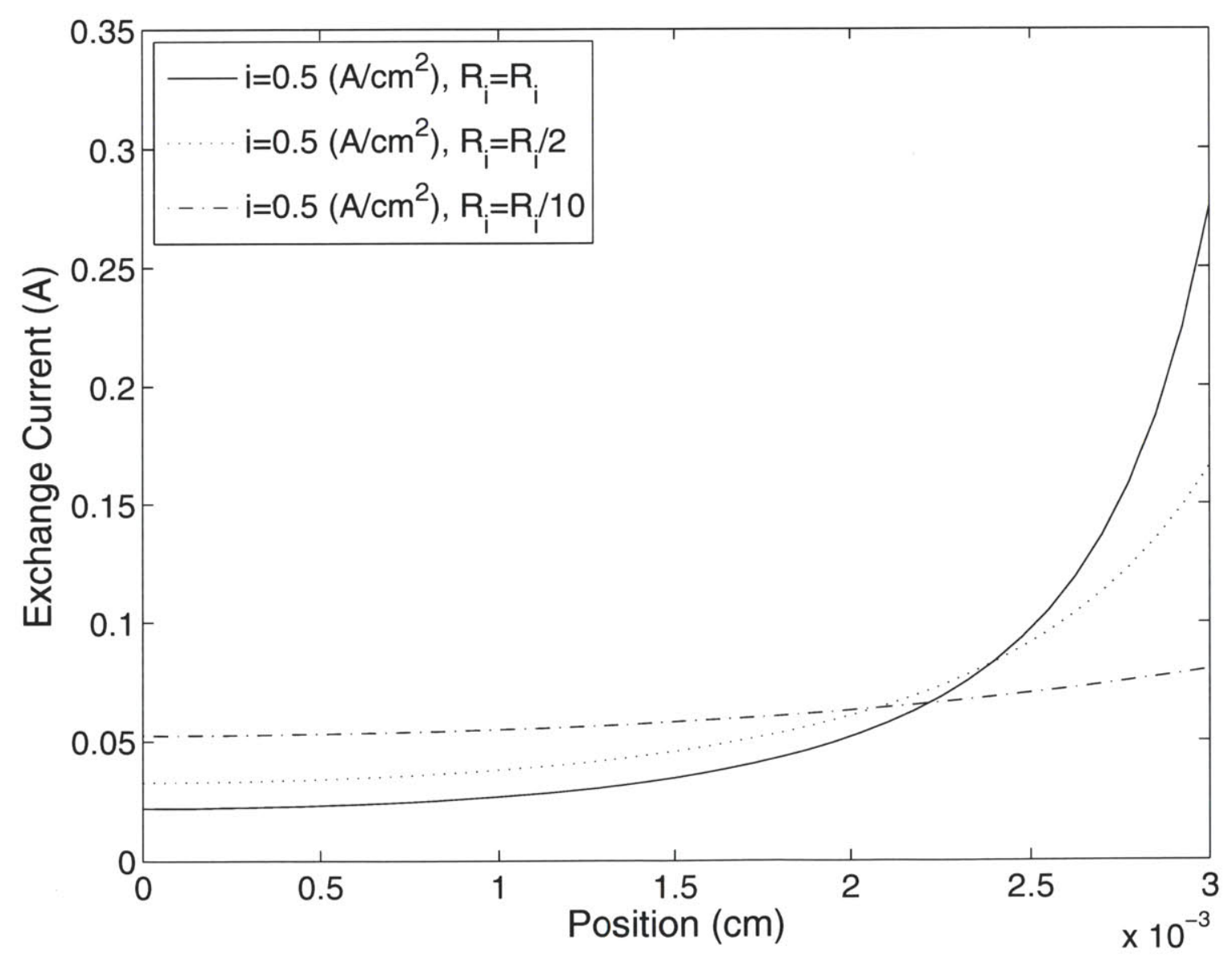

Figure 3-3: Exchange current as a function of position in the electrode as simulated with network model with $x=0$ corresponding to the current collector. 


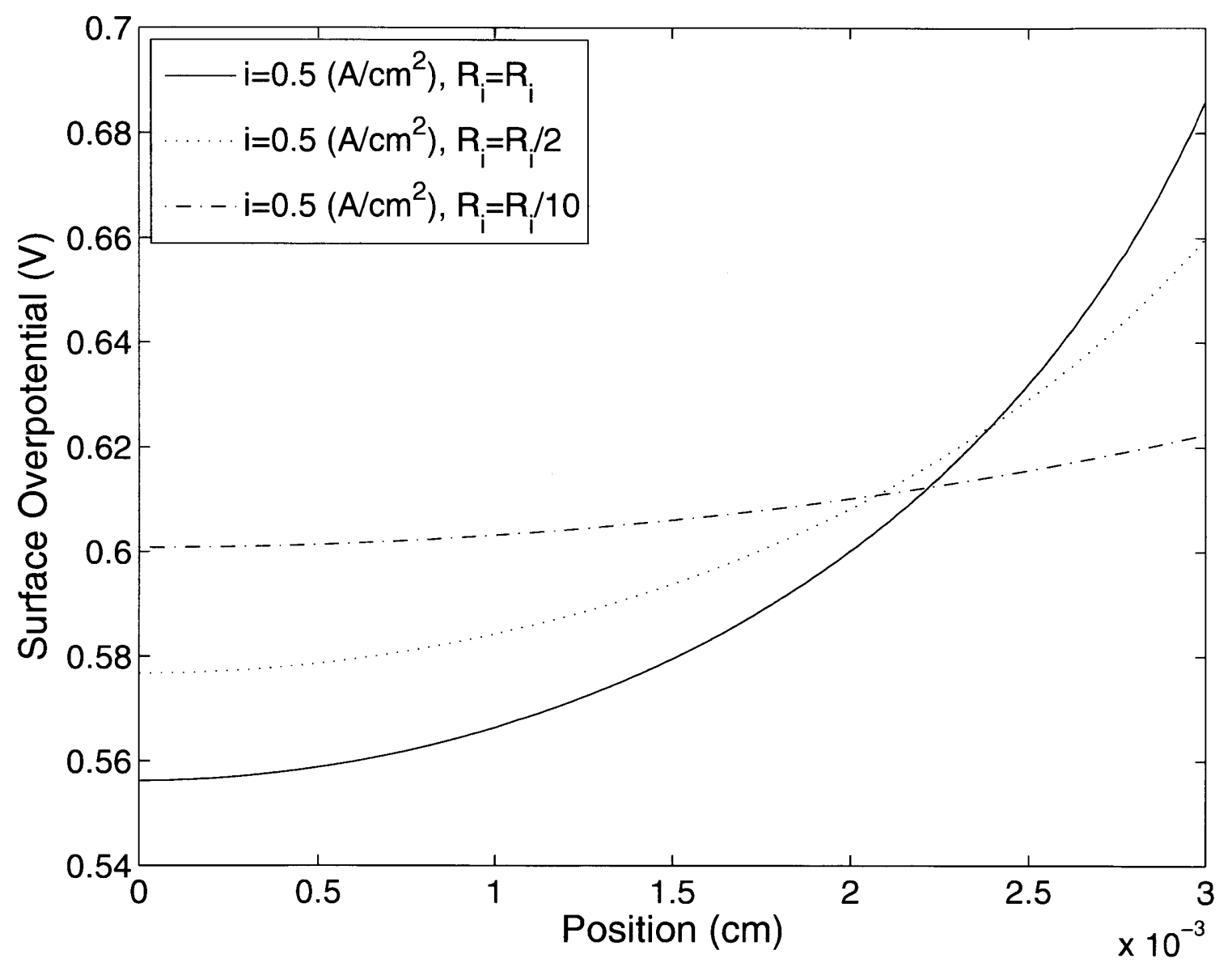

Figure 3-4: Overpotenial as a function of position in the electrode as simulated with network model with $x=0$ corresponding to the current collector. 
This dual-regime arrangement can be visualized with an electrical network diagram shown in Figure 3-2. Resistors, $R_{e}$ and $R_{i}$, represent the electronic resistance, and the ionic resistance components, in the electrode respectively. Non-linear elements, $Z_{c t}$, represent the faradaic charge transfer process. The charge transfer process is governed by Butler-Volmer kinetics:

$$
i=i_{o}\left[\exp \left(\frac{\alpha_{a} \cdot F \cdot \eta}{R \cdot T}\right)-\exp \left(\frac{-\alpha_{c} \cdot F \cdot \eta}{R \cdot T}\right)\right]
$$

where $\eta$ is the surface overpotenial defined by the potential difference between the ionic and electronic regimes at each point in the electrode, $i_{o}$ is the exchange current density, $F$, is Faraday's constant, $R$, is the universal gas constant, and $\alpha_{a}$ and $\alpha_{c}$ are the anodic and cathodic transfer coefficients, respectively. Solving this electrical network model for a given current then gives a current distribution in the electrode, shown in Figure 3-3, and an overpotenial distribution in the electrode, shown in Figure 3-4. Each of these figures plots three solutions to the model. The solid line uses typical parameters for a DMFC anode, the dotted line uses the same parameters, but with the ionic resistance reduced by half, and the dash-dotted line uses the original parameters but with the ionic resistance decreased by a factor of 10. The results in Figure 3-3 clearly show that a disproportionate fraction of the current is transferred in the third of the electrode closest to the membrane. Similarly, the distribution of overpotenial in Figure 3-4 shows that the overpotenial at the membrane, which is the total overpotenial for the electrode, is significantly larger than the overpotential at the current collector. Additionally, the reduction of ionic resistance will make great improvements in evening out the distributions of both current and potential in the electrode. This illustrates the consequences of not being able to satisfy $F R_{5}$ independently from the other FRs. If $F R_{5}$ were independent, then the Nafion content could be increased to drop ionic resistance and even out reaction rate in the electrode.

The electrical network model described above relies on a finite number of charge transfer paths to approximate electrode behavior. If the charge transfer paths are made infinitesimally small, then the governing differential equations for the model can be formulated and, depending on the constituent electrical elements, solved. Models of this type are generally referred to as transmission line models because of their origins in modeling telegraph wires [26]. 


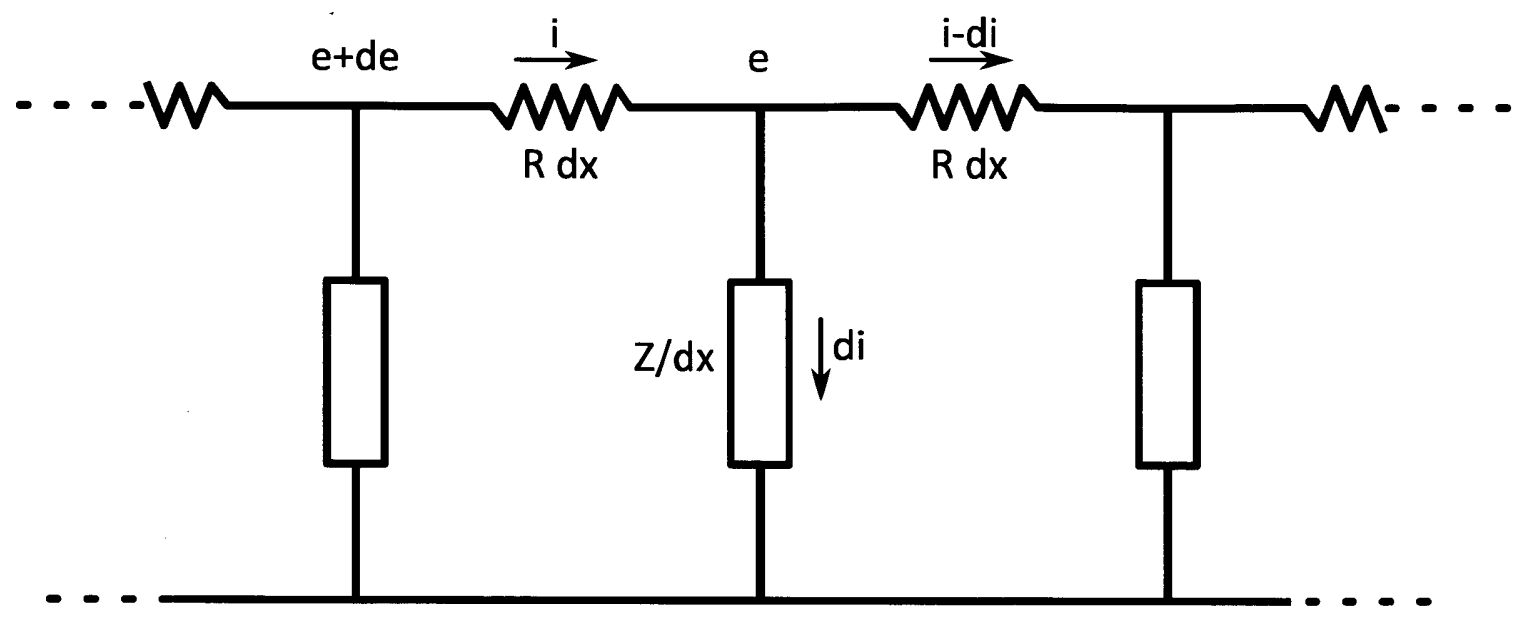

Figure 3-5: Differential section of a transmission line.

If the electronic resistance in the electrode is assumed to be negligible, then the network model takes the form of a transmission line model. The derivation that follows was presented by De Levie [27] on the modeling of porous and rough electrodes. De Levie attributes the use of this model for electrochemical systems to Daniel'-Bek [28] and Weisselberg [29]. If the charge-transfer elements in the transmission line are treated as differential elements with length $\mathrm{d} x$ of the total transmission line length, then from Figure 3-5 it can easily be seen that:

$$
\mathrm{d} e=-i R \mathrm{~d} x
$$

and

$$
\mathrm{d} i=-\frac{e}{Z} \mathrm{~d} x
$$

Note that $R$ is the ionic resistance per unit length and $Z$ is the impedance of the charge-transfer interface per unit length. These equations can be rearranged such that:

$$
\frac{\mathrm{d} e}{\mathrm{~d} x}+i R=0
$$

and 


$$
\frac{\mathrm{d} i}{\mathrm{~d} x}+\frac{e}{Z}=0
$$

If $R$ and $Z$ are independent of $x$ then Equations 3.6 and 3.7 can be combined to obtain

$$
\begin{aligned}
& \frac{\mathrm{d}^{2} e}{\mathrm{~d} x^{2}}-\frac{R}{Z} e=0 \\
& \frac{\mathrm{d}^{2} i}{\mathrm{~d} x^{2}}-\frac{R}{Z} i=0
\end{aligned}
$$

If $R$ and $Z$ are functions of $x$, then the governing equations for the transmission line are

$$
\begin{aligned}
& \frac{\mathrm{d}^{2} e}{\mathrm{~d} x^{2}}-\frac{\mathrm{d} \ln R}{\mathrm{~d} x} \frac{\mathrm{d} e}{\mathrm{~d} x}-\frac{R}{Z} e=0 \\
& \frac{\mathrm{d}^{2} i}{\mathrm{~d} x^{2}}+\frac{\mathrm{d} \ln Z}{\mathrm{~d} x} \frac{\mathrm{d} i}{\mathrm{~d} x}-\frac{R}{Z} i=0
\end{aligned}
$$

When $Z$ is approximated as a simple resistance, $R^{\prime}$, the solution for the system of Equations 3.8 and 3.9 for the boundary conditions

$$
\begin{array}{rlrl}
x & =0 & e & =e_{0} \\
x & =l & \frac{\mathrm{d} e}{\mathrm{~d} x} & =0
\end{array}
$$

is

$$
e=e_{0} \frac{\cosh (\rho x-\rho l)}{\cosh (\rho l)}
$$

where

$$
\rho=\left(\frac{R}{R^{\prime}}\right)^{\frac{1}{2}}
$$

From Equation 3.6, the total current entering the pore at $x=0$ is

$$
i_{0}=-\frac{1}{R}\left(\frac{\mathrm{d} e}{\mathrm{~d} x}\right)_{e=0}=\frac{\rho e_{0}}{R} \tanh (\rho l)
$$


Then the pore behaves roughly like a resistance

$$
\frac{e_{0}}{i_{0}}=\left(R R^{\prime}\right)^{\frac{1}{2}} \operatorname{coth}(\rho l)
$$

From Equation 3.17, the current is inversely proportional to $\sqrt{R \cdot R^{\prime}}$ in porous electrodes, whereas on flat electrodes, current is inversely proportional to only $R^{\prime}$. This is an indication of the importance of the ionic resistance in porous electrodes. Once an electrode is thick enough for ionic resistance to be a factor, ionic resistance has equal importance to that of charge transfer resistance.

Another significant result from this derivation is the concept of the penetration depth, $1 / \rho$. Electrodes much thicker than this depth behave as if they were semi-infinitely deep. An electrode can be considered semi-infinite if $l \cdot \rho \gg 1$, then the potential in the electrode is:

$$
e=e_{0} \exp (-\rho x)
$$

and by applying Equation 3.6 the current is:

$$
i=\frac{e_{0} \rho}{R} \exp (-\rho x)
$$

From the integration of Equation 3.19, $63 \%$ of the total current is calculated to be exchanged within the penetration depth. Either extending the penetration depth by increasing conductance, or reducing the electrode thickness, leads to a much better distribution of current and a lower voltage drop in the electrode.

The linearized charge transfer resistance, $R^{\prime}$, of a faradaic reaction is inversely proportional to the catalyst area available. Assuming that specific catalyst area is a constant, then $R^{\prime}$ is also inversely proportional to catalyst loading, $m_{P t}$. Therefore,

$$
l \cdot \rho \propto \frac{1}{\sqrt{R^{\prime}}} \propto \sqrt{m_{P t}}
$$

so even if adding catalyst does not increase electrode thickness, the imbalance of reaction rates within the electrode will increase. If, as is likely, the electrode thickness is also proportional to catalyst loading, it has an even stronger influence on imbalanced reaction rates in the electrode: 


$$
l \cdot \rho \propto m_{P t}^{3 / 2}
$$

This means that as catalyst is added to the electrode, the reaction distribution within the catalyst becomes less uniform. In order to maintain electrode performance, ionic conductivity must be increased. This is the origin of the influence of $D P_{1}$, catalyst loading, on $F R_{5}$, the need for ionic conductivity, which is shown the design matrix in Table 3.2.

\subsubsection{Applications to Impedance Spectroscopy}

The transmission line model results with linearized charge transfer are especially important results for impedance spectroscopy. Since the applied oscillation amplitude is small in impedance spectroscopy, even non-linear interfacial processes behave like linear impedances. As a result, the overall impedance for a porous electrode can be found by substituting the interfacial impedance, $Z$, into Equation 3.17 in place of the charge transfer resistance, $R^{\prime}$.

$$
Z_{0}=(R Z)^{1 / 2} \operatorname{coth}\left[\left(\frac{R}{Z}\right)^{1 / 2} l\right]
$$

Equation 3.22 can be used to predict the impedance response of porous electrodes by simply substituting in the assumed interfacial impedance model.

One instructive case is to assume that the interfacial impedance can be represented by a capacitance, which assumes that the catalyst is an ideally-polarizable, or blocking, electrode with a uniform smooth surface. In this case, the capacitance value, $C$, is linearly related to the catalyst area. The impedance of a capacitor is:

$$
Z=\frac{-j}{\omega C}
$$

so the overall impedance then becomes:

$$
Z_{0}=\sqrt{\frac{-R \cdot j}{\omega C}} \operatorname{coth}(l \sqrt{\omega R C \cdot j})
$$

Taking the square root of the imaginary unit in Equation 3.24 yields:

$$
Z_{0}=\sqrt{\frac{R}{2 \omega C}}(1-j) \operatorname{coth}\left(\sqrt{\frac{\omega l^{2} R C}{2}}(1+j)\right)
$$


Expanding the hyperbolic cotangent in Equation 3.25 into its real and imaginary parts yields:

$$
Z_{0}=\sqrt{\frac{R}{2 \omega C}}(1-j) \frac{\sinh \left(\sqrt{2 \omega l^{2} R C}\right)-j \sin \left(\sqrt{2 \omega l^{2} R C}\right)}{\cosh \left(\sqrt{2 \omega l^{2} R C}\right)-\cos \left(\sqrt{2 \omega l^{2} R C}\right)}
$$

The complex terms in Equation 3.26 can be combined to yield:

$$
Z_{0}=\sqrt{\frac{R}{2 \omega C}} \frac{\sinh \left(\sqrt{2 \omega l^{2} R C}\right)-\sin \left(\sqrt{2 \omega l^{2} R C}\right)-j\left[\sinh \left(\sqrt{2 \omega l^{2} R C}\right)+\sin \left(\sqrt{2 \omega l^{2} R C}\right)\right]}{\cosh \left(\sqrt{2 \omega l^{2} R C}\right)-\cos \left(\sqrt{2 \omega l^{2} R C}\right)}
$$

The real and imaginary parts can be easily separated into

$$
Z_{0}^{\prime}=\sqrt{\frac{R}{2 \omega C}} \frac{\sinh \left(\sqrt{2 \omega l^{2} R C}\right)-\sin \left(\sqrt{2 \omega l^{2} R C}\right)}{\cosh \left(\sqrt{2 \omega l^{2} R C}\right)-\cos \left(\sqrt{2 \omega l^{2} R C}\right)}
$$

and

$$
Z_{0}^{\prime \prime}=-\sqrt{\frac{R}{2 \omega C}} \frac{\sinh \left(\sqrt{2 \omega l^{2} R C}\right)+\sin \left(\sqrt{2 \omega l^{2} R C}\right)}{\cosh \left(\sqrt{2 \omega l^{2} R C}\right)-\cos \left(\sqrt{2 \omega l^{2} R C}\right)}
$$

Nyquist plots of this model, calculated with the parameters listed in Table 3.4, are shown in Figure 3-6, including the effects of changing the model parameters, $R, C$, and $l$ from their baseline values. In the high frequency region, the hyperbolic sine term dominates over the sine term so that the real and imaginary parts are roughly equal in magnitude leading to the $-45^{\circ}$ slope in the Nyquist plot. In the low frequency region, the real part approaches a limit of $R \cdot l / 3$ while the imaginary term approaches infinity, as shown in Figure 3-6a.

Capacitance plots are another common representation of impedance data and are used extensively to present the impedance results in Chapter 5. The transmission line model can be used to highlight some of the properties that make capacitance plots useful. Figure 3-7 shows capacitance plots of the same impedance spectra as in Figure 3-6, which are formed by replacing the imaginary axis, $Z^{\prime \prime}$, with an effective capacitance axis calculated as

$$
C_{e f f}=\frac{1}{\omega Z^{\prime \prime}}
$$




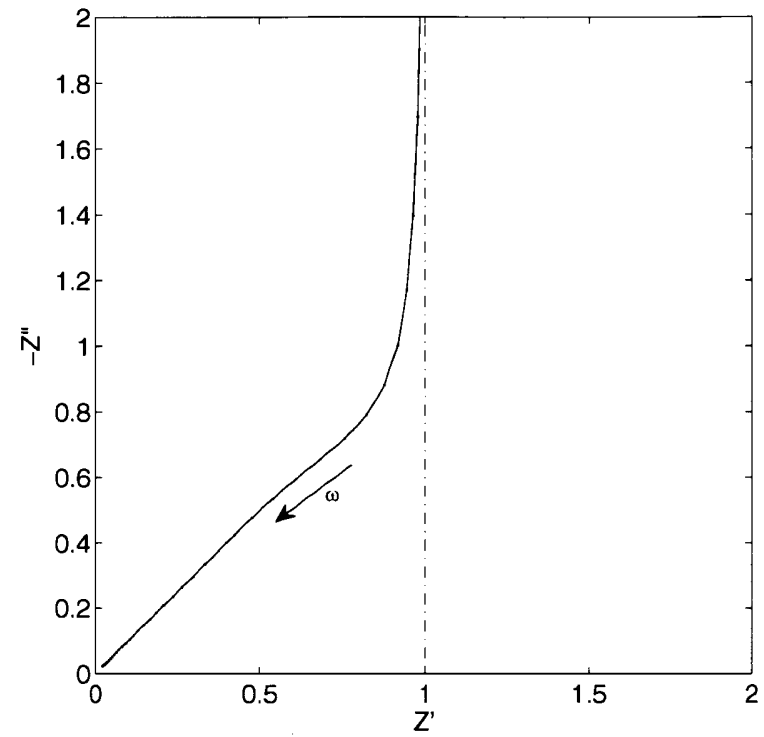

(a)

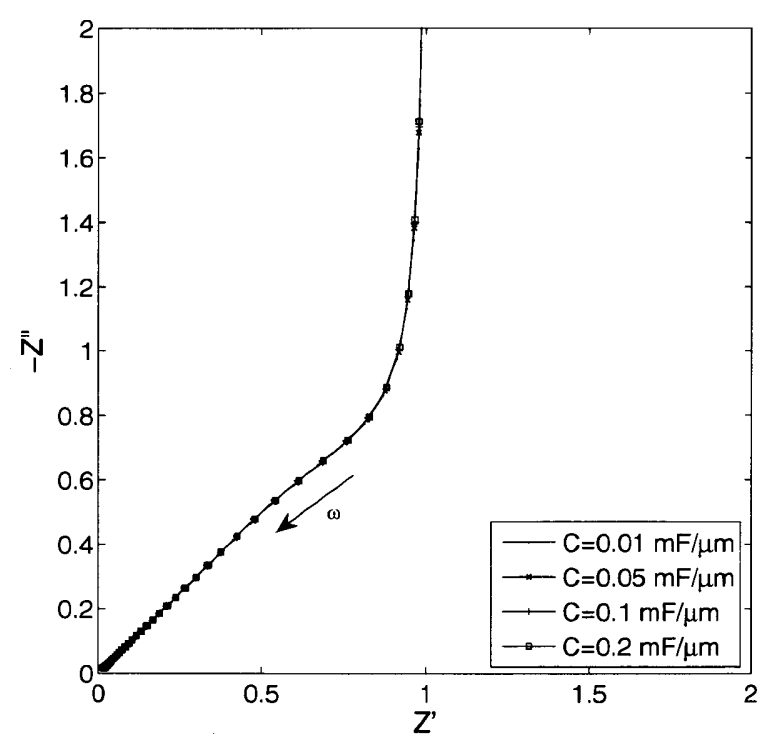

(c)

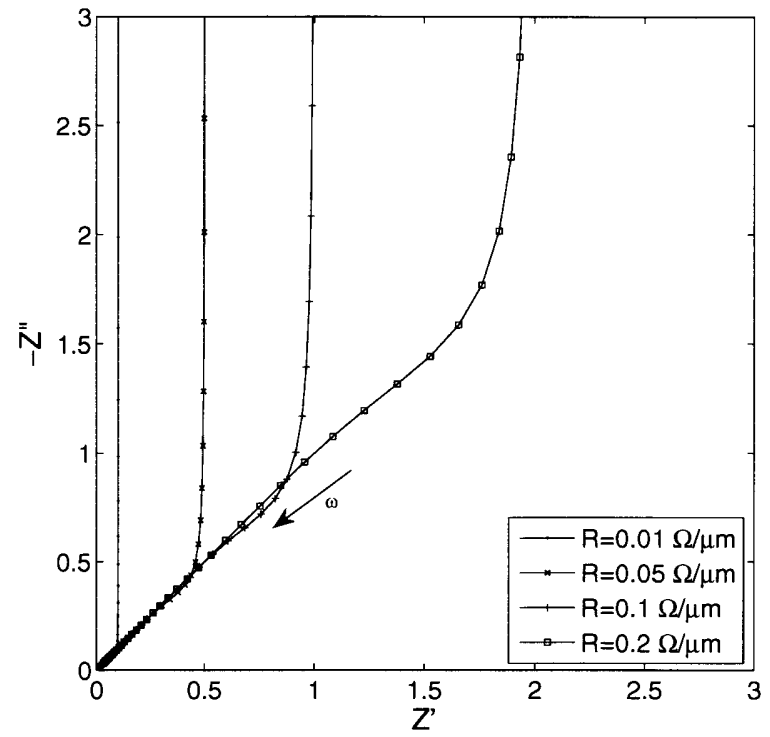

(b)

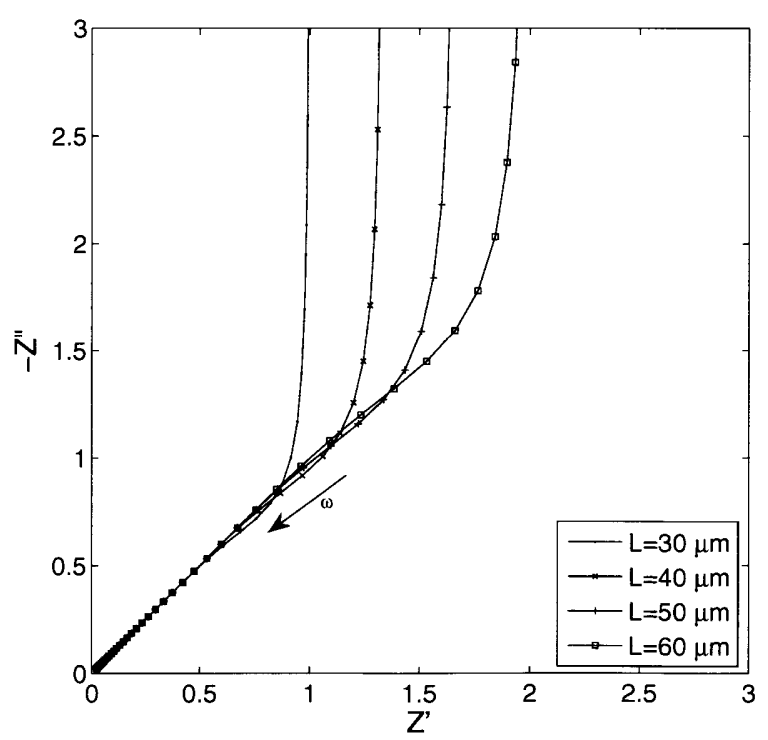

(d)

Figure 3-6: Calculated Nyquist plots of a transmission line model shown with (a) the $R \cdot l / 3$ real limit; (b) several $R$ values; (c) several $C$ values; and (d) several $L$ values. 


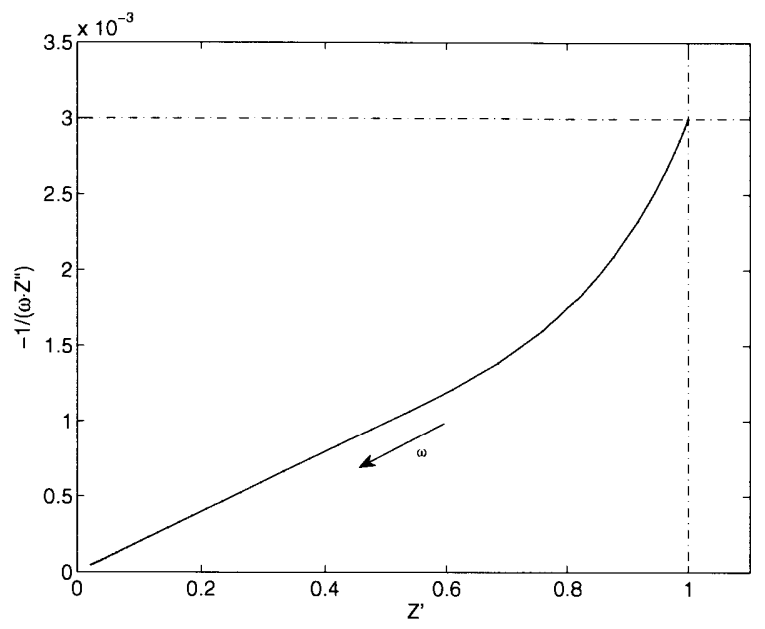

(a)

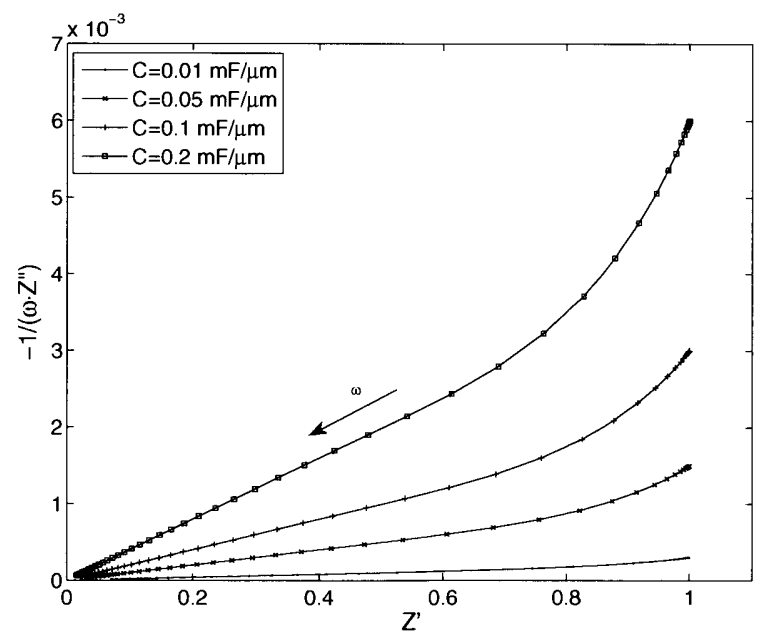

(c)

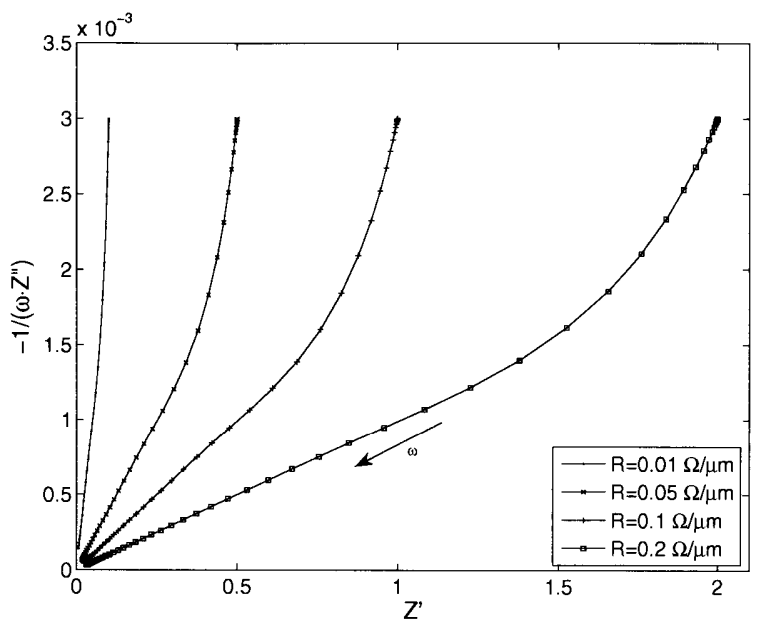

(b)

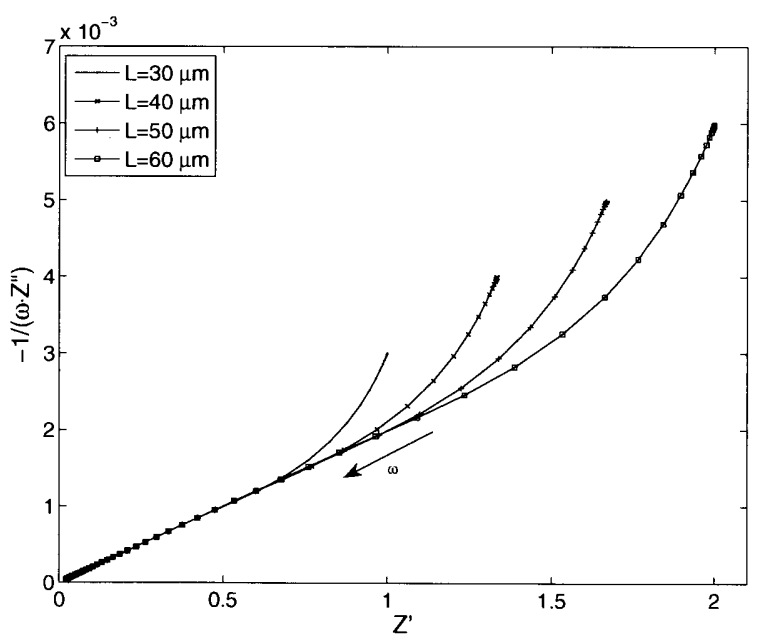

(d)

Figure 3-7: Calculated capacitance plots of a transmission line model shown with (a) the $R \cdot l / 3$ real limit and $C \cdot l$ capacitance limit; (b) several $R$ values; (c) several $C$ values; and (d) several $L$ values . 


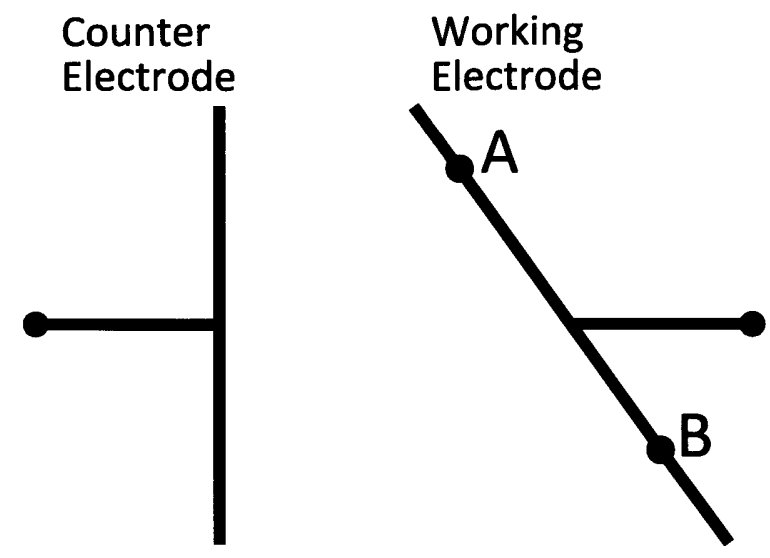

Figure 3-8: Example cell illustrating the origins of the constant phase element.

By substituting Equation 3.29 into Equation 3.30, the effective capacitance of the transmission line model can be determined.

$$
C_{e f f}=\sqrt{\frac{2 C}{\omega R}} \frac{\cosh \left(\sqrt{2 \omega l^{2} R C}\right)-\cos \left(\sqrt{2 \omega l^{2} R C}\right)}{\sinh \left(\sqrt{2 \omega l^{2} R C}\right)+\sin \left(\sqrt{2 \omega l^{2} R C}\right)}
$$

As frequency goes to zero, the effective capacitance of the transmission line reaches a limit of $C \cdot l$. This limit is shown in Figure $3-7$ a. The transmission line model with capacitive charge transfer indicates that electrode ionic resistance and electrode active area can be quickly evaluated by looking at the low-frequency behavior, shown in Nyquist and capacitance plots.

\subsubsection{Constant Phase Element}

While capacitive charge-transfer is a useful special case, the real behavior of porous electrodes does not match a purely capacitive model. There is, however, still no faradaic reaction taking place in the experiments presented in this thesis, so the more realistic model should still be applicable to a blocking electrode. The constant-phase element (CPE) is an excellent option to model more realistic blocking electrode behavior. The CPE is commonly used to model blocking electrode surfaces that are porous and/or physically or kinetically nonuniform [30, $31,32,33]$. All of these conditions apply in this case.

One way to understand the origins of the constant phase element behavior for nonuniform electrodes is to consider a two electrode cell, in which the working electrode is not parallel to the counter electrode, as shown in Figure 3-8. In this example, the length of 
Table 3.4: Baseline parameters used in transmission line models.

\begin{tabular}{|c|c|}
\hline Parameter & Value \\
\hline \hline Electrode thickness, $l$ & $30 \mu \mathrm{m}$ \\
\hline Ionic Resistance per unit thickness, $R$ & $0.1 \Omega / \mu \mathrm{m}$ \\
\hline Capacitance per unit thickness, $C$ & $0.1 \mathrm{mF} / \mu \mathrm{m}$ \\
\hline CPE Capacitance per unit thickness, $T$ & $0.1 \mathrm{mF} / \mu \mathrm{m} \cdot \mathrm{s}^{1-\phi}$ \\
\hline CPE Phase exponent, $\phi$ & 0.8 \\
\hline
\end{tabular}

electrolyte, and thus the resistance, traversed by a charge carrier, is much smaller if the charge is transferred at point $\mathrm{A}$ than if the charge is transferred at point B. As a result, there is a real component to the blocking electrode's behavior in addition to the expected capacitive behavior. On a Nyquist plot, this manifests itself as a straight line with the slope determined by the amount of variation. The resulting impedance of a constant phase element is

$$
Z_{C P E}=\frac{1}{T(j \omega)^{\phi}}
$$

where $T$ is a capacitance-like term proportional to the area of electrode available, and $\phi$ is the phase exponent that can vary from 0 to 1 , and determines the angle of the CPE's Nyquist plot. If $\phi$ is equal to 1 , then the element is a pure capacitance and if $\phi$ is equal to 0 , then the element is a pure resistance. For rough and porous surfaces, the constant phase behavior can be derived from a fractal representation of the electrode surface with the phase exponent being related to the degree of fractal surface magnification or the degree of roughness. For a perfectly flat single-crystal surface, the phase exponent would be 1 , as the fractal dimension increases, i.e. for a rougher surface, the phase exponent decreases, though the functional relationship for this dependence is not clear [31, 32].

Using a constant phase element in the charge-transfer path of the transmission line model, the impedance from Equation 3.22 becomes:

$$
Z_{0}=\sqrt{\frac{R}{T(\omega j)^{\phi}}} \operatorname{coth}\left(l \sqrt{R T \cdot(\omega j)^{\phi}}\right)
$$

Nyquist plots of the modified transmission line model calculated with the parameters listed in Table 3.4 are shown in Figure 3-9, including the effects of changing the model parameters, $\phi, R, T$, and $l$ from their baseline values. Unlike Equation 3.24, neither the 


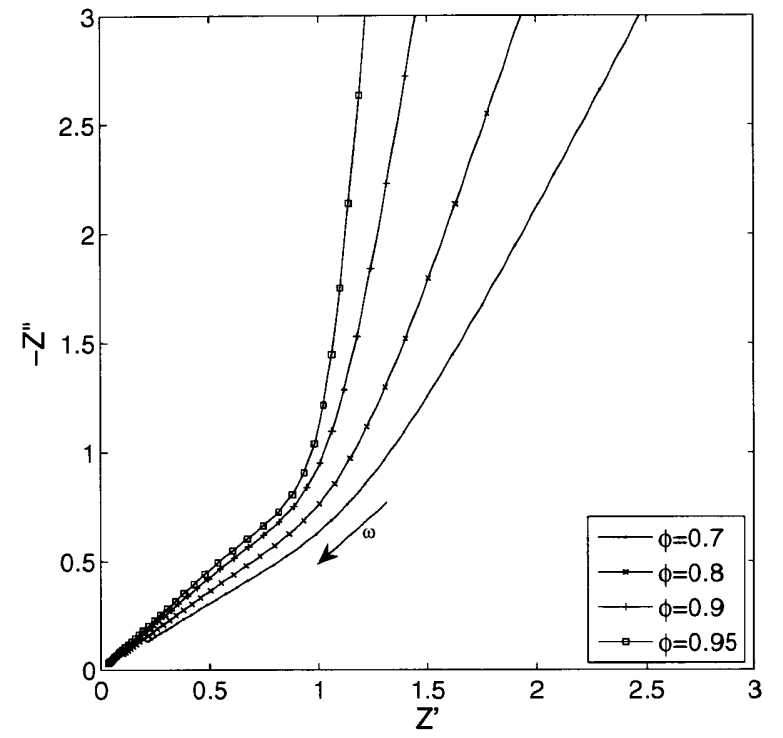

(a)

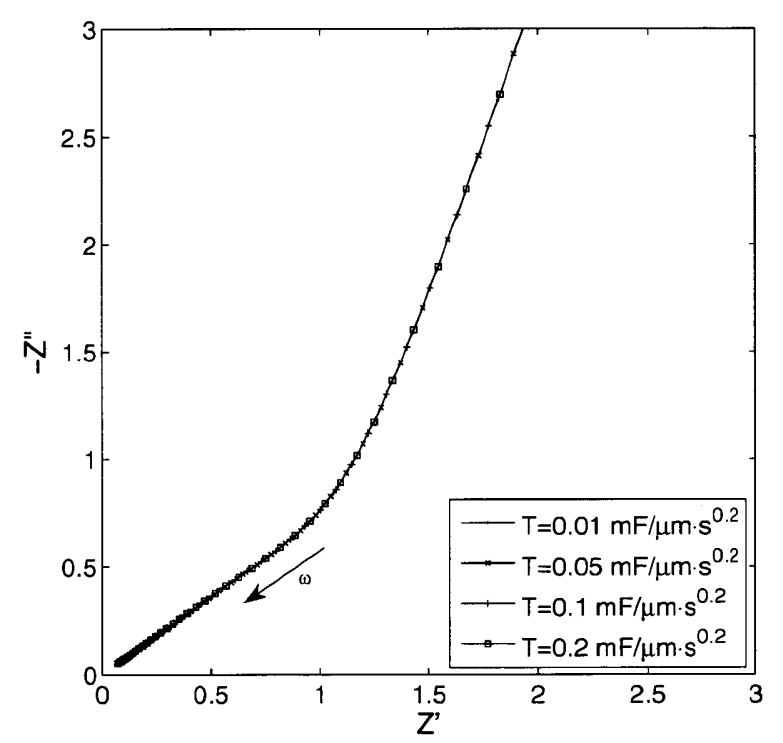

(c)

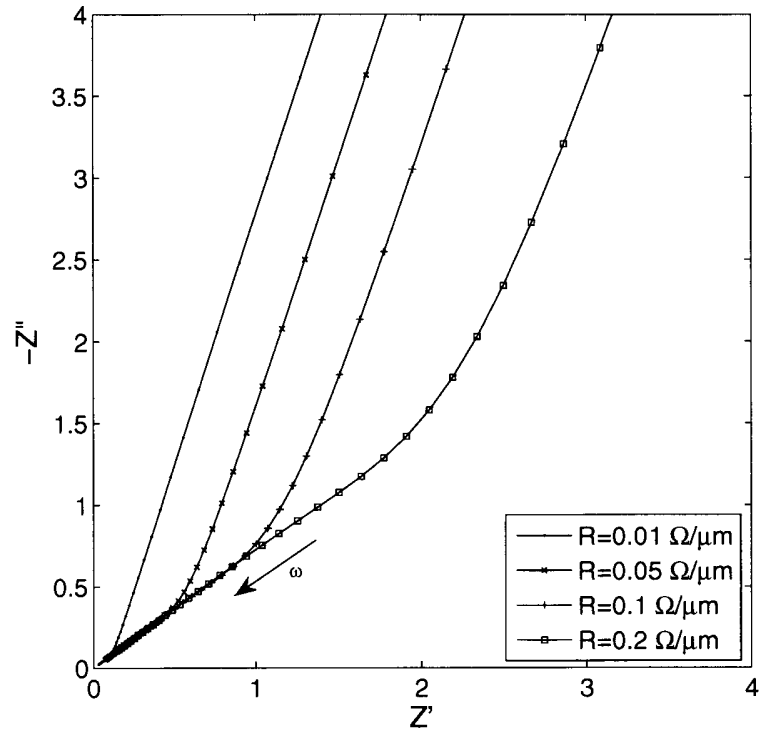

(b)

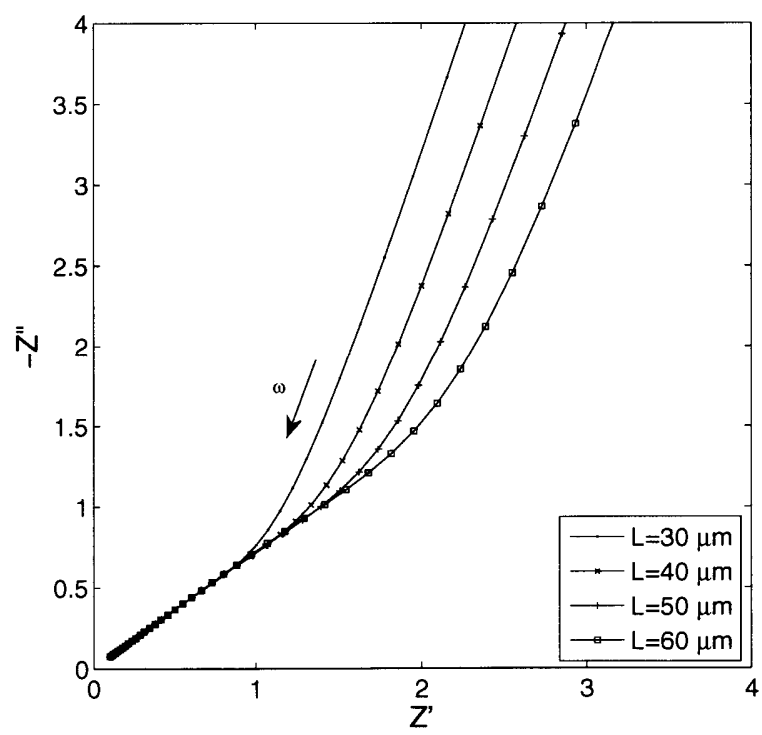

(d)

Figure 3-9: Calculated Nyquist plots of a transmission line model using constant phase element charge transfer and shown with (a) several $\phi$ values; (b) several $R$ values; (c) several $C$ values; and (d) several $L$ values . 
real nor the imaginary portions of Equation 3.33 are bounded as the frequency approaches zero. Similarly, if the imaginary portion of the impedance is transformed into an effective capacitance, it too grows without bound as the frequency approaches zero.

Some of the behavior, however, does carry over from the capacitive charge transfer case to the constant phase charge transfer case. Capacitance plots of the CPE transmission line, shown in Figure 3-10, do show an asymptotic-like behavior at low-frequencies. As with the capacitive transmission line, the capacitance-like term, $T$, in the CPE transmission line controls the level of this low-frequency asymptote in the capacitance plot, as shown in Figure 3-10c. Similarly, the ionic resistance term in the CPE transmission line still has the effect of shifting the low-frequency asymptote of the Nyquist plot, shown in Figure 3-9b, along the real axis without changing the low-frequency asymptote of the capacitance plot, shown in Figure 3-10b. The length of the transmission line, $l$, has a similar influence on the CPE version as it does on the capacitive version: increasing transmission line length shifts the low-frequency asymptote to the right in the Nyquist plot, shown in Figure 3-9d, and shifts the low-frequency asymptote up in the capacitance plot, shown in Figure 3-10d. This means that the low-frequency behavior of Nyquist and capacitance plots remains an effective way to judge electrode ionic resistance and electrode active area, even with non-ideal blocking electrodes.

\subsubsection{Catalyst Loading Limit}

The existence of a catalyst loading limit for fuel cell electrodes has been well documented in literature $[2,17]$. Furthermore, experimental results described in Section 5.2.3 show that the catalyst loading limit is observable using impedance spectroscopy of blocking electrodes. The transmission line model, however, cannot predict this loading limit using either capacitive charge transfer or constant phase element charge transfer. In the transmission line model, the addition of catalyst to an electrode comes in the form of increasing the thickness of an electrode, $l$; increasing the capacitance term, $C$, or the capacitance-like term, $T$; or a combination of the two. The effect of the capacitance term on the impedance spectra, predicted by both the capacitive and constant-phase charge transfer models, is shown in Figures 3-6c, 3-7c, 3-9c, and 3-10c. For the capacitive charge transfer model, there is a linear increase in the capacitance limit, as predicted by Equation 3.31. Similarly for the constant-phase charge transfer model, there is a linear progression in the low-frequency 


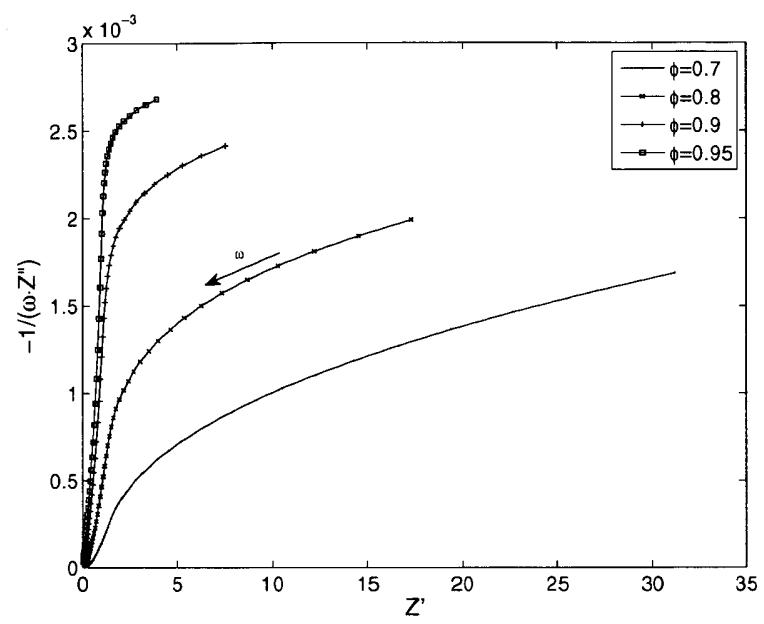

(a)

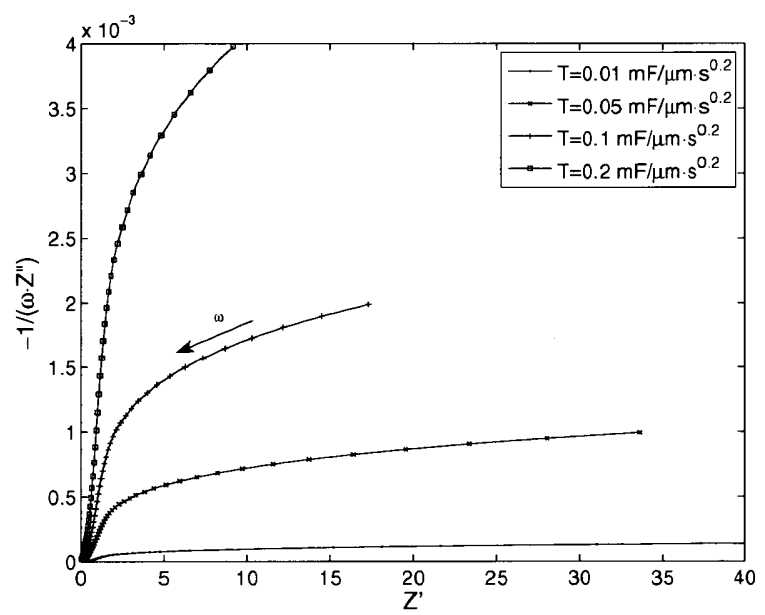

(c)

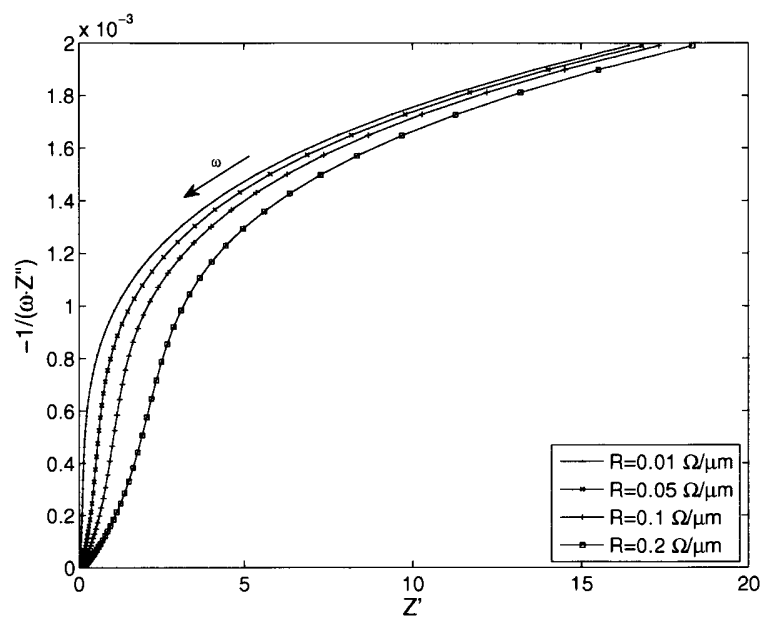

(b)

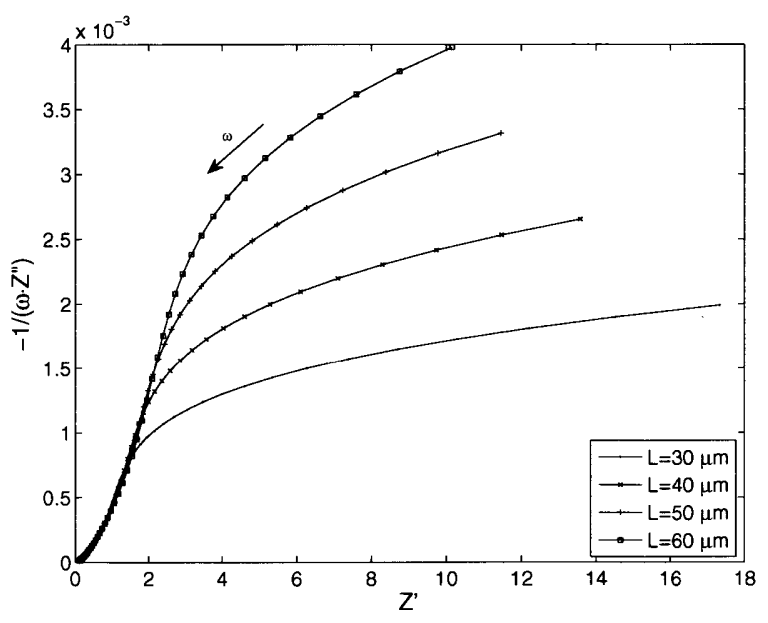

(d)

Figure 3-10: Calculated capacitance plots of a transmission line model using constant phase element charge transfer and shown with (a) several $\phi$ values; (b) several $R$ values; (c) several $C$ values; and (d) several $L$ values . 
behavior, shown in the capacitance plot. The effect of electrode thickness on the predicted impedance spectra of both models can be seen in Figures 3-6d, 3-7d, 3-9d, and 3-10d. Again, for the capacitance charge-transfer model, Equations 3.28 and 3.31 predict linear increases in both the real impedance limit and the effective capacitance limit, which are confirmed in Figures 3-6d and 3-7d. The low-frequency asymptote of the constant-phase charge transfer model also shows a linear progression in both the Nyquist and capacitance plots. For all cases, there is no amount of loading that would change this trend.

This indicates that the catalyst loading limit is either not exclusively due to poor ionic conductivity in the fuel cell electrode, or that ionic conductivity changes in a more nuanced fashion as catalyst loading increases. One possibility is that as an electrode uses higher catalyst loading, the uniformity of the electrode decreases. For instance, high-loading electrodes may result in the dispersed Nafion concentrating near the Nafion membrane, which would lead to the useful catalyst near the membrane being blocked while the catalyst far from the membrane has even less ionic connection than before.

If the catalyst loading limit is simply a result of behavior not included in the transmission line model, such as fuel diffusion or changes in electronic conduction, then a more advanced model may be able to predict the catalyst loading limit. This possibility was not explored as part of this thesis.

\subsection{Model of Structured Fuel Cell Electrodes}

The electrical network model for a DMFC anode and the transmission line model with resistive charge transfer illustrate that ionic resistance has a strong influence on reaction rate uniformity within the anode. The transmission line with capacitive and constant phase charge transfer shows that the effect of ionic resistance on porous blocking electrodes can be easily evaluated using Nyquist and capacitance plots of impedance data. The question to be answered is whether or not the proposed structured electrode design will, in fact, reduce ionic resistance in the cell. To investigate this, a two-dimensional electrode model was developed to address the influence of Nafion posts on electrode behavior. 

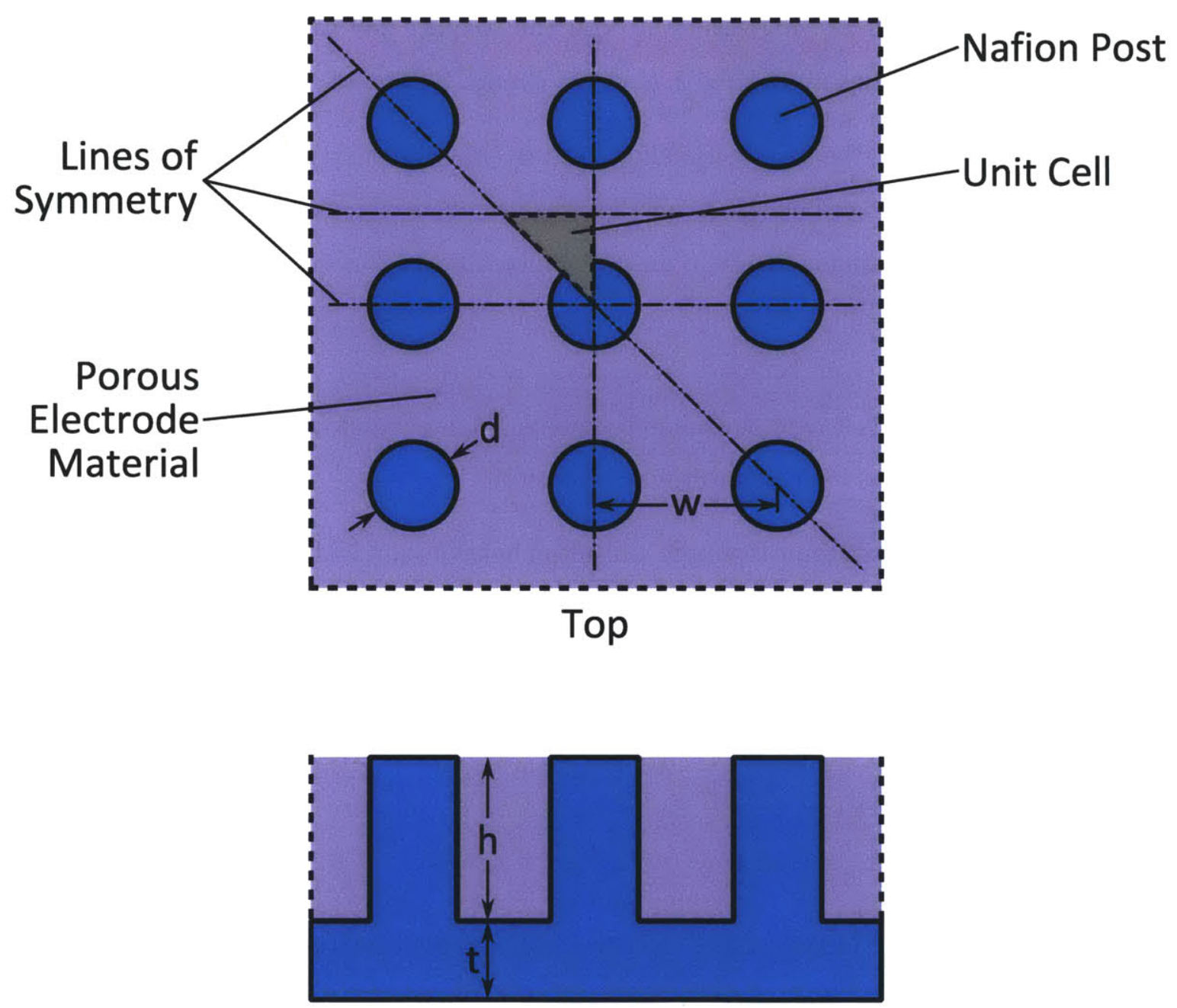

Side

Figure 3-11: Top and side views of the structured electrode showing lines of symmetry and the resulting unit cell of the post pattern. 


\subsubsection{Two-Dimensional Model Architecture}

The structured electrode uses an array of Nafion posts to improve ionic conductivity. The electrodes created in this thesis use a square array of posts, but other arrangements are possible. As shown in Figure 3-11, the structured electrode geometry is defined by four parameters: the diameter of the posts, $d$; the center-to-center spacing of the posts, $w$; the thickness of the electrode, $h$; and the thickness of the membrane, $t$. To simplify the model, lines of symmetry are drawn on the post array to identify the unit cell of the periodic structure, which is shown in Figure 3-11. By symmetry, the boundary of the unit cell must have a no flux boundary condition. To further reduce the model, it is assumed that the unit cell is also radially symmetric about the center of the Nafion post. This simplification allows the structured electrode to be modeled as a two-dimensional structure by looking at a radial slice from the center of the Nafion post to the edge of the unit cell boundary.

The radial slice that is simulated in this model is shown in Figure 3-12. Like the onedimensional model before it, this model consists of two parallel domains: one for electronic conduction and one for ionic conduction. The electronic domain consists only of the porous electrode material between the posts, and is shown in green in Figure 3-12. The ionic plane consists of the bulk Nafion that makes up the posts and the membrane, shown in blue, and the porous electrode region that fills the space between posts, shown in purple. The electronic and ionic domains are connected, in the porous electrode region only, through a charge-transfer impedance, just as in the one-dimensional case. In the structured case, the model also includes a portion of the Nafion membrane, to allow the concentrated current expected in the Nafion post to disperse evenly in the membrane.

The domains of this model are discretized into nodes. Between adjacent nodes within each domain is a resistor, the value of which is calculated based on the size of the differential element between those nodes:

$$
\begin{aligned}
& R_{r}=\frac{\rho_{i} \cdot \mathrm{d} r}{r \cdot \mathrm{d} \theta \cdot \mathrm{d} z} \\
& R_{z}=\frac{\rho_{i} \cdot \mathrm{d} z}{r \cdot \mathrm{d} \theta \cdot \mathrm{d} r}
\end{aligned}
$$

where $R_{r}$ is the resistor in the radial direction, $R_{z}$ is the resistor in the axial direction, $\rho_{i}$ is the resistivity at current node, $r$ is the radial position of the node, $\mathrm{d} r$ is the radial node 


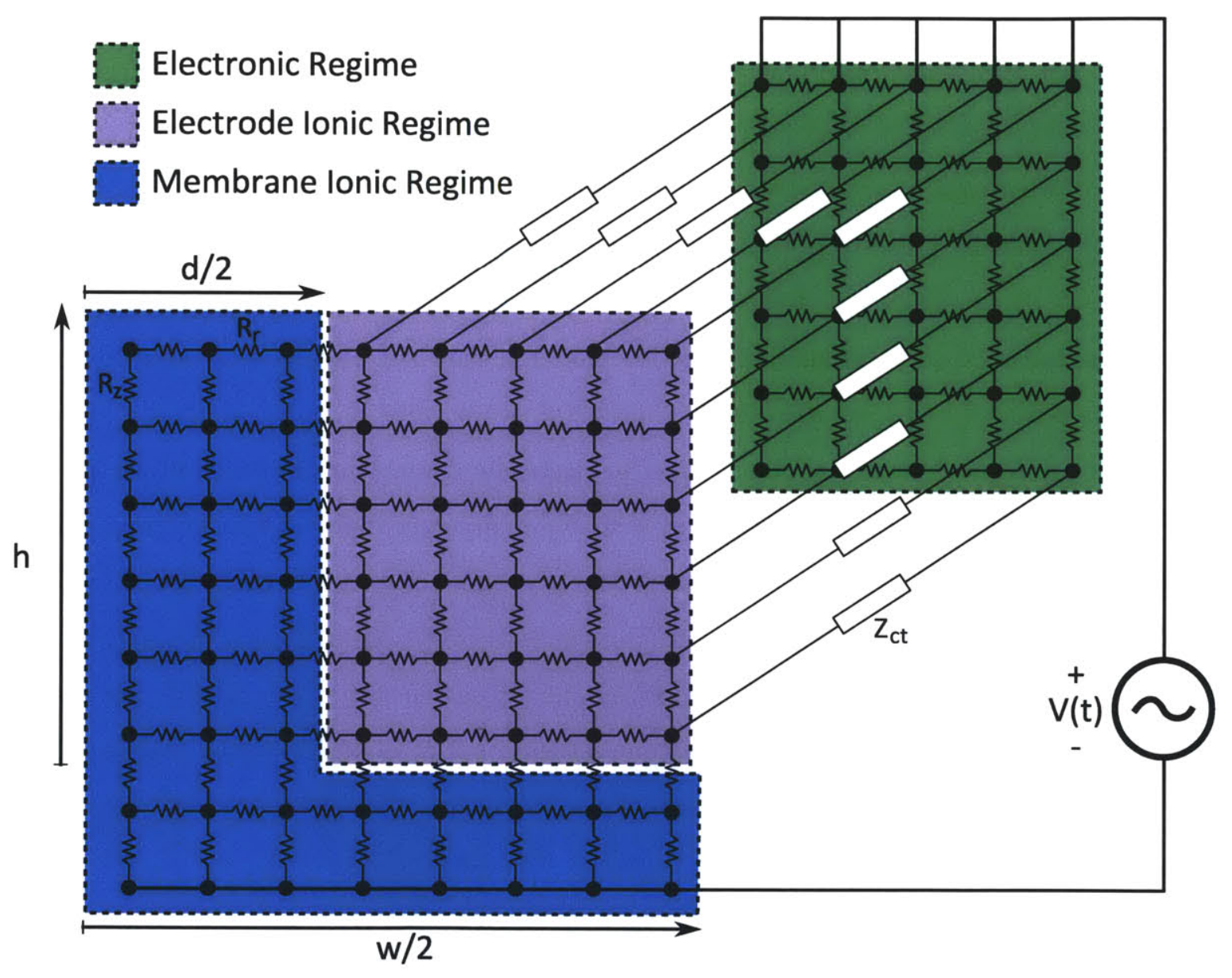

Figure 3-12: Node structure used in two-dimensional model 
spacing, $\mathrm{d} z$ is the axial node spacing, and $\mathrm{d} \theta$ is the angle subtended by the unit cell.

The nodes in the porous electrode also have a connection to the matching node in the opposite domain. This connection is a generalized impedance, also calculated based on the size of the element containing the node. For the calculations presented in this thesis, the generalized impedance is assumed to be a constant-phase element, as was used in the one-dimensional model, so the impedance between ionic and electronic nodes, $Z_{c t}$ is:

$$
Z_{c t}=\frac{1}{T_{i}(\omega \cdot j)^{\phi}}
$$

where $\omega$ is the excitation frequency, $\phi$ is the $\mathrm{CPE}$ phase exponent, and $T_{i}$ is the $\mathrm{CPE}$ capacitance at this node. The CPE capacitance, $T_{i}$, is based on the size of the element surrounding the node.

$$
T_{i}=\xi \cdot r \cdot \mathrm{d} \theta \cdot \mathrm{d} r \cdot \mathrm{d} z
$$

The capacitance per unit volume, $\xi$, is a measure of the capacitance loading in the porous electrode material.

Once the model is appropriately discretized into an electrical network, the solution is found using nodal analysis. In this method, the state of the network is defined by a vector containing the potential at each node in the network. A system of equations is formed by applying Kirchhoff's Current Law at each node. That is, the sum of the current entering a node is zero. Because of the regular connection pattern between the nodes, the system matrix for this network has a banded structure, making the matrix inversion process computationally easy. To simulate impedance spectroscopy, the system matrix is solved at each frequency to find the total impedance of the electrode. Total impedance is found by assuming that each unit cell is connected in parallel with the other unit cells. Thus, the impedance is simply normalized to the overall electrode area as:

$$
Z_{\text {full }}=Z_{\text {unit }} \frac{A_{\text {unit }}}{A_{\text {full }}}
$$

Much of the utility for this model will be in comparing results with the standard electrode. To make a fair comparison, the standard electrode calculation is done with the same model, but the post diameter is set to zero. Catalyst loading is set by the thickness of the 
Table 3.5: Baseline parameters used in the two-dimensional structured electrode model.

\begin{tabular}{|c||c|}
\hline Parameter & Value \\
\hline \hline Bulk Nafion Resistivity, $\rho_{N a f}$ & $7 \cdot 10^{4} \Omega \cdot \mu m$ \\
\hline Porous Electrode Ionic Resistivity, $\rho_{I}$ & $3 \cdot 10^{6} \Omega \cdot \mu m$ \\
\hline Porous Electrode Electronic Resistivity, $\rho_{E}$ & $3 \cdot 10^{-3} \Omega \cdot \mu m$ \\
\hline Capacitance per unit Electrode Volume, $\xi$ & $2 \cdot 10^{-12} \frac{F}{\mu m^{3} \cdot s^{1-\phi}}$ \\
\hline CPE Phase, $\phi$ & 0.8 \\
\hline Post Spacing, $w$ & $175 \mu m$ \\
\hline Post Diameter, $d$ & $75 \mu m$ \\
\hline Electrode Thickness, $h$ & $60 \mu m$ \\
\hline Membrane Thickness, $t_{m}$ & $100 \mu m$ \\
\hline
\end{tabular}

standard electrode. The actual thickness of the structured cell, $h_{s t r}$, is selected to keep the total catalyst loading constant, and is dependent on the electrode geometry as:

$$
h_{s t r}=h_{s t d} \frac{w^{2}}{w^{2}-d^{2}}
$$

where $h_{s t d}$ is the thickness of the standard electrode, $w$ is the center-to-center post spacing of the array, and $d$ is the post diameter.

\subsubsection{Structured Model Results}

All results from this model use the baseline parameter values shown in Table 3.5, unless the values are otherwise specified. Figure 3-13 shows Nyquist and capacitance plots of the impedance spectra calculated using the two-dimensional model for both a structured case and the standard electrode case. From the transmission line model, shown in Figure 3$9 \mathrm{~b}$, the position of the low-frequency asymptote in the Nyquist plot is a good indicator of ionic resistance in the electrode. In Figure 3-13a, the structured model clearly shifts the low-frequency asymptote of the Nyquist plot toward lower real impedance values, indicating that the ionic resistance of the electrode has decreased. A look at the capacitance plot of these impedance spectra, in Figure 3-13b, shows that the low-frequency asymptote of the structured electrode is far below the asymptote of the standard cell, which corresponds to a drop in total electrode capacitance, as shown in Figure 3-10c.

The results from the two-dimensional model, in Figure 3-13, seem to match quite well with the form of the spectra from the one-dimensional transmission line model in Figures 3-9 and 3-10. The results of the two-dimensional structured model can therefore be approxi- 


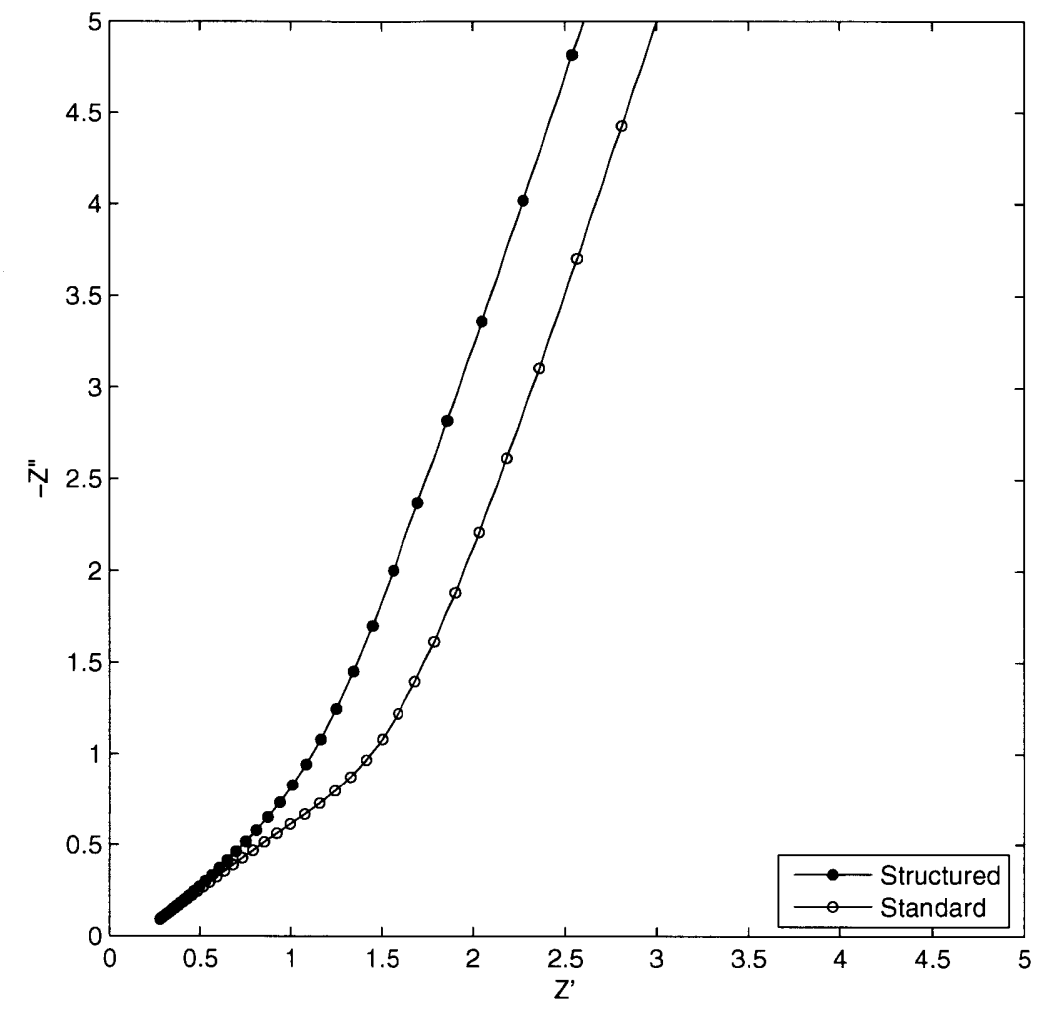

(a)

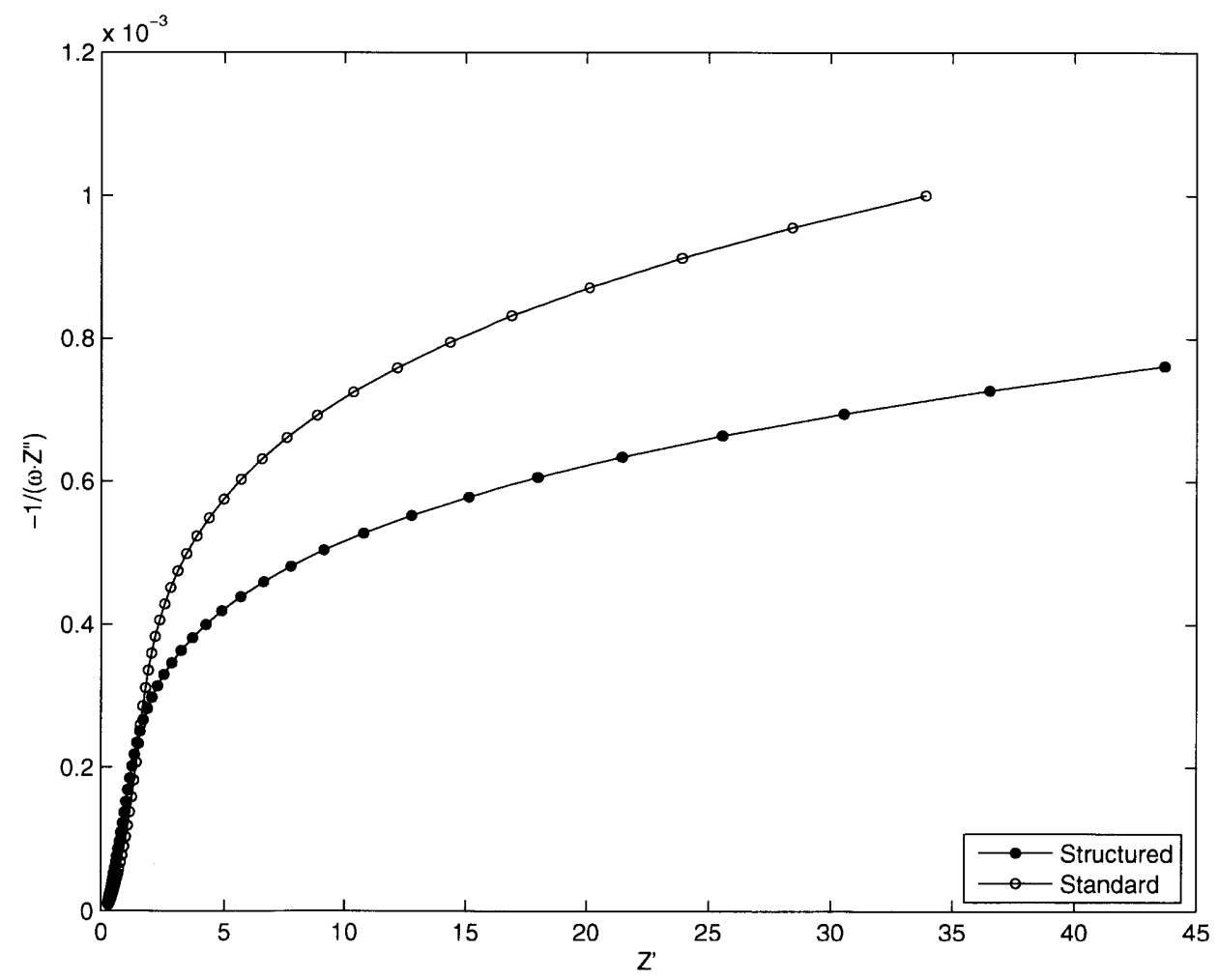

(b)

Figure 3-13: Nyquist (a) and capacitance (b) plots comparing standard and structured electrodes. 
Table 3.6: Transmission line model parameters that approximate the impedance spectra simulated with the two-dimensional electrode model.

\begin{tabular}{|c|c|c|c|c|c|}
\hline & $R_{s}(\Omega)$ & $R_{i}(\Omega)$ & $T\left(F / \mu m^{3} s^{1-\phi}\right)$ & $\phi$ & Norm. Error $(\Omega)$ \\
\hline \hline Standard Electrode & 0.2493 & 7.0192 & .0028 & .8 & 0.0301 \\
\hline Structured Electrode & 0.1816 & 4.4429 & 0.0023 & 0.7987 & 0.4540 \\
\hline
\end{tabular}

mated with the one-dimensional transmission model. The Nyquist and capacitance plots of the two-dimensional model are shown again in Figure 3-14, but this figure also includes a plot of one-dimensional transmission line models that approximate the two-dimensional results. Not surprisingly, the standard model is matched almost perfectly by the transmission line model. Since the features in this model are one-dimensional, the model based on the one-dimensional assumption works quite well. The transmission line model also matches the structured model quite well, though not as well as the unstructured model. The major deviations between the structured model and the transmission line approximation occur in the high-frequency range of the Nyquist plot, shown in Figure 3-14a. The parameters used in the transmission line model to approximate the two structured models are shown in Table 3.6, along with the normalized error for each spectra. These data serve to confirm the conclusions drawn by inspection from the Nyquist and capacitance plots: the structured electrodes have significantly lower ionic resistance and slightly lower capacitance values.

\subsubsection{Effect of Material Properties}

The two-dimensional model uses several material properties to define the electrode. These include the effective capacitance density, $\xi$, the effective ionic resistivity of the porous electrode material, $\rho_{I}$, the effective electronic resistivity of the electrode, $\rho_{E}$, the ionic resistivity of the bulk Nafion, $\rho_{N a f}$, and the phase exponent of the constant phase element charge transfer, $\phi$. For changes in most of these values, the structured cell model behaves exactly as expected in view of the transmission line model.

The electronic resistivity of the electrode is so low that it has no influence at all on the model behavior. A change in the effective capacitance density results predominantly in a shift of the impedance spectrum along the frequency axis. It is also clearly evident in the low-frequency asymptote of the capacitance plots in Figure 3-15, yet in all cases the structured electrode's asymptote is below that of the standard electrode. Variations in bulk Nafion resistivity shift the series resistance, which is made up predominately of the 


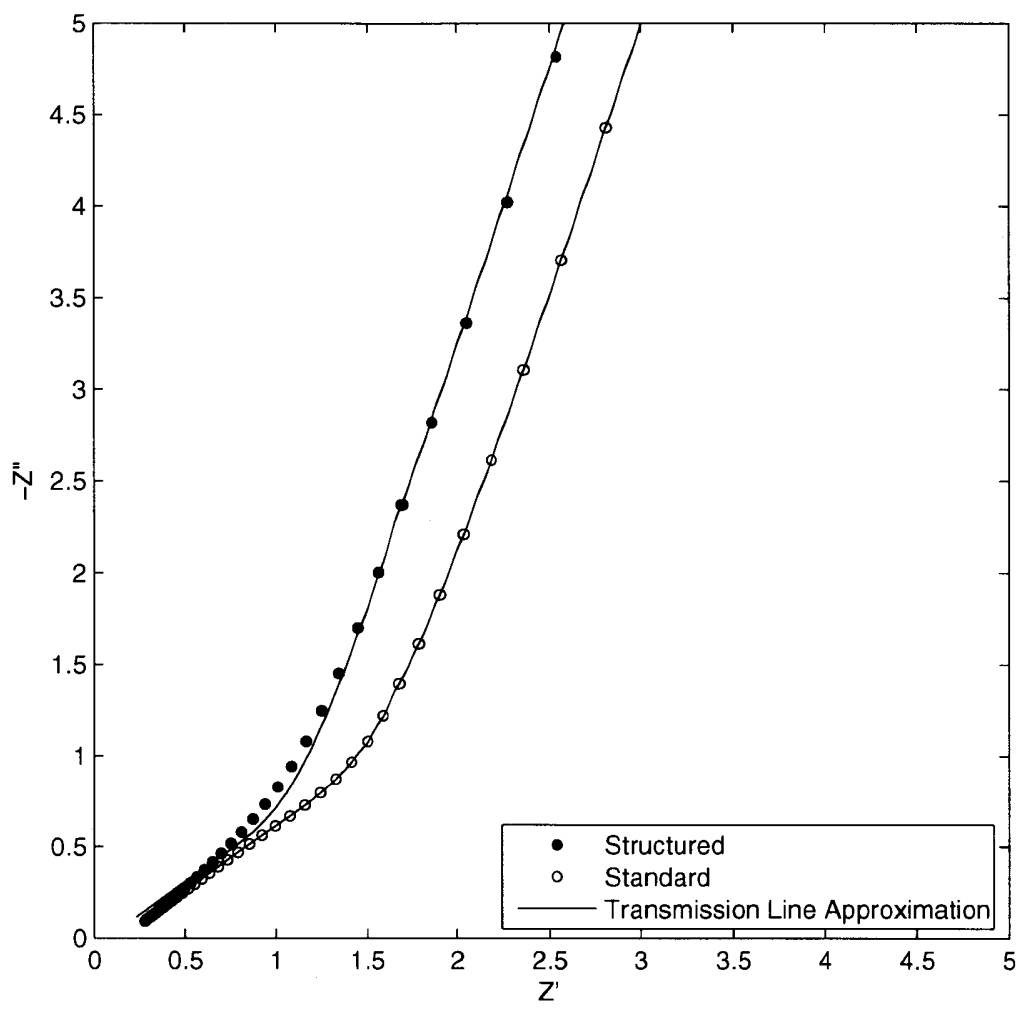

(a)

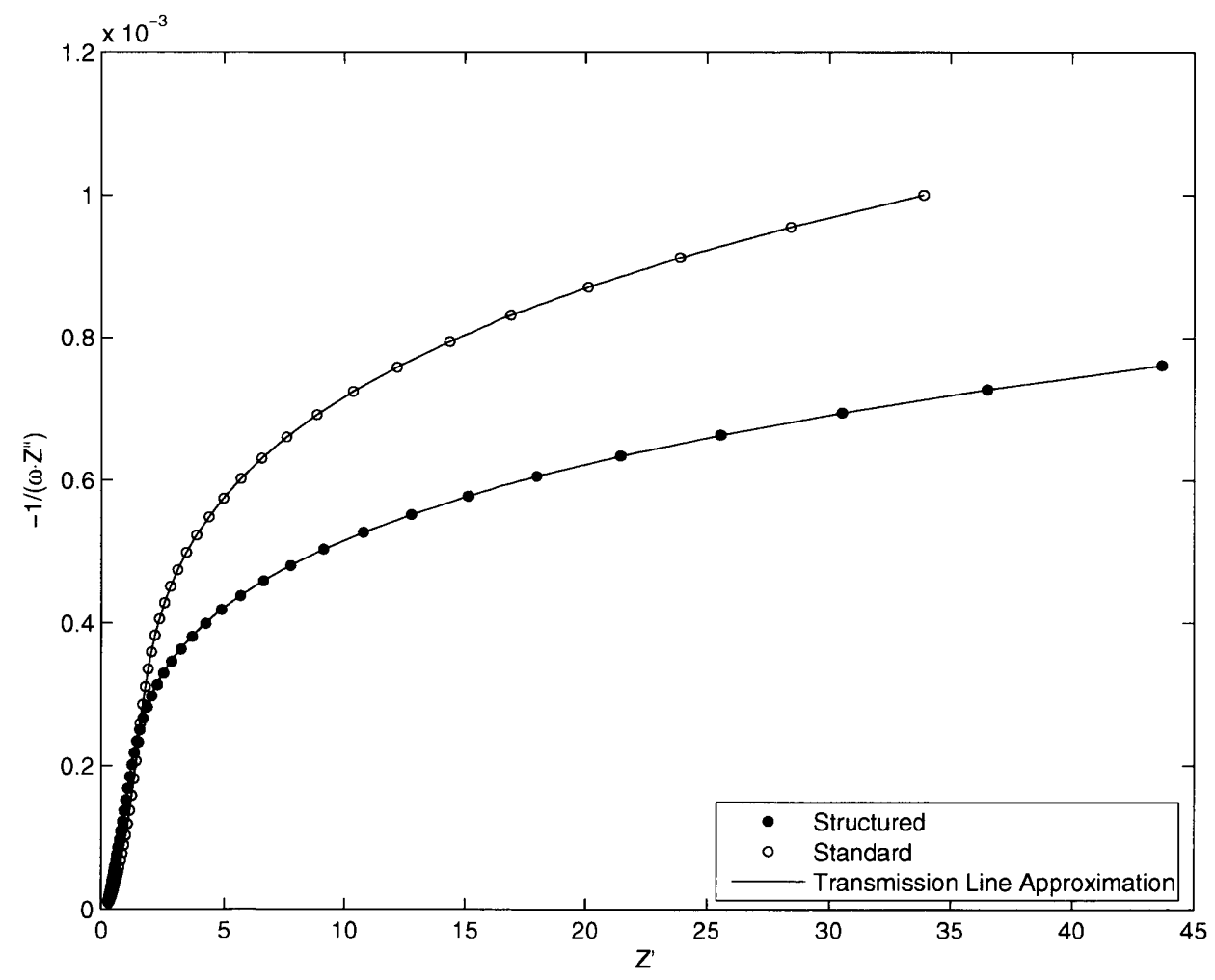

(b)

Figure 3-14: Nyquist (a) and capacitance (b) plots comparing standard and structured electrodes. 


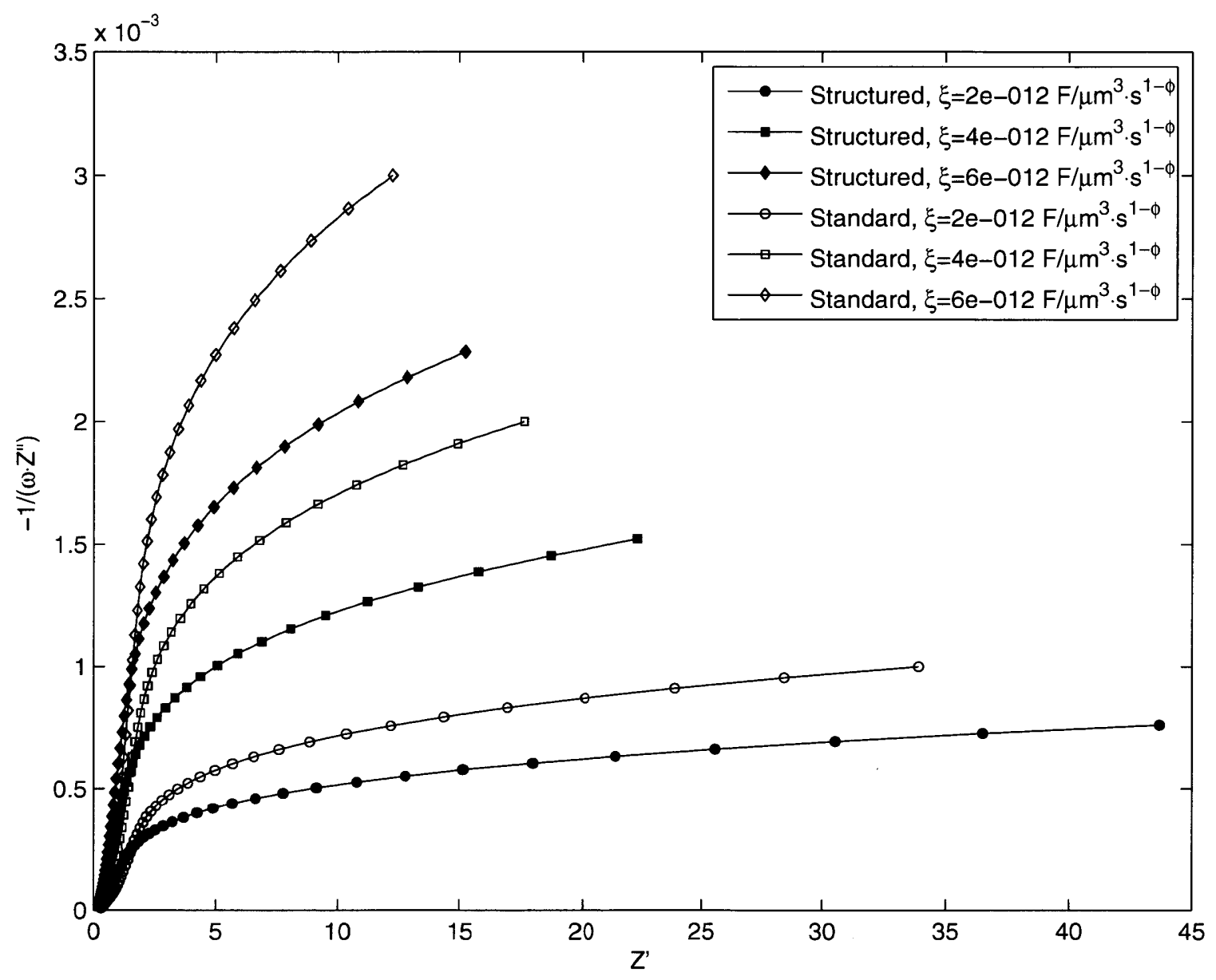

Figure 3-15: Capacitance plot showing the effect of changing capacitance density within the electrode material on the impedance spectra predicted from the structured electrode model. 
membrane included in this model, but not the transmission line model. The phase exponent of the constant phase element behaves essentially exactly as it does in the transmission line model. It controls the slope of the low-frequency asymptote in the Nyquist plot.

The effect of the porous electrode's resistivity on the behavior of the structured electrode is much less trivial. As with the resistance term in the transmission line model, changing ionic resistivity in the structured electrode does not appreciably change the capacitance value seen in the low-frequency asymptote of the capacitance plot in Figure 3-16b. The Nyquist plot in Figure 3-16a, however, shows that as ionic resistivity in the electrode increases, the benefit of adding the structure to the electrode also increases. This point can be more clearly seen in Figure 3-17, which plots the approximate ionic resistance value for the standard and structured cells as electrode ionic resistivity varies.

\subsubsection{Effect of Structured Electrode Geometry}

The main purpose of the two-dimensional model is to explore the impact of structure geometry on the ionic resistance in a fuel cell electrode. The addition of Nafion posts to the electrode is intended to reduce ionic resistance in the electrode and improve the performance of catalyst material not directly adjacent to the membrane. The addition of the posts, however, does require that part of the already effective catalyst material near the membrane be displaced in order to make room for the posts. One major question is whether the benefit of the posts is sufficient to outweigh the displacement of effective catalyst material. One way to look at this question is to run the electrode model with several different post diameters. The Nyquist and capacitance plots calculated using a range of post diameters are shown in Figures $3-18 \mathrm{~b}$ and $3-18 \mathrm{a}$, respectively. The most striking of the features in these plots is that the low-frequency asymptote in the Nyquist plot, shown in Figure 3-18a, moves fairly little with a change in post diameter, suggesting that the post diameter has little influence on ionic resistance. In fact, the difference between the standard electrode and any of the structured electrodes is much larger than the differences between the structured electrode with different sized posts. This trend is clear in looking at the approximate ionic resistance term as a function of post diameter in Figure 3-19. As diameter decreases, ionic resistance in the structured electrode increases slightly, yet remains far below that of the standard electrode until the post diameter drops below a critical diameter. Below this critical diameter, approximately $25 \mu \mathrm{m}$ in this case, a decrease in post diameter leads to significant increases 


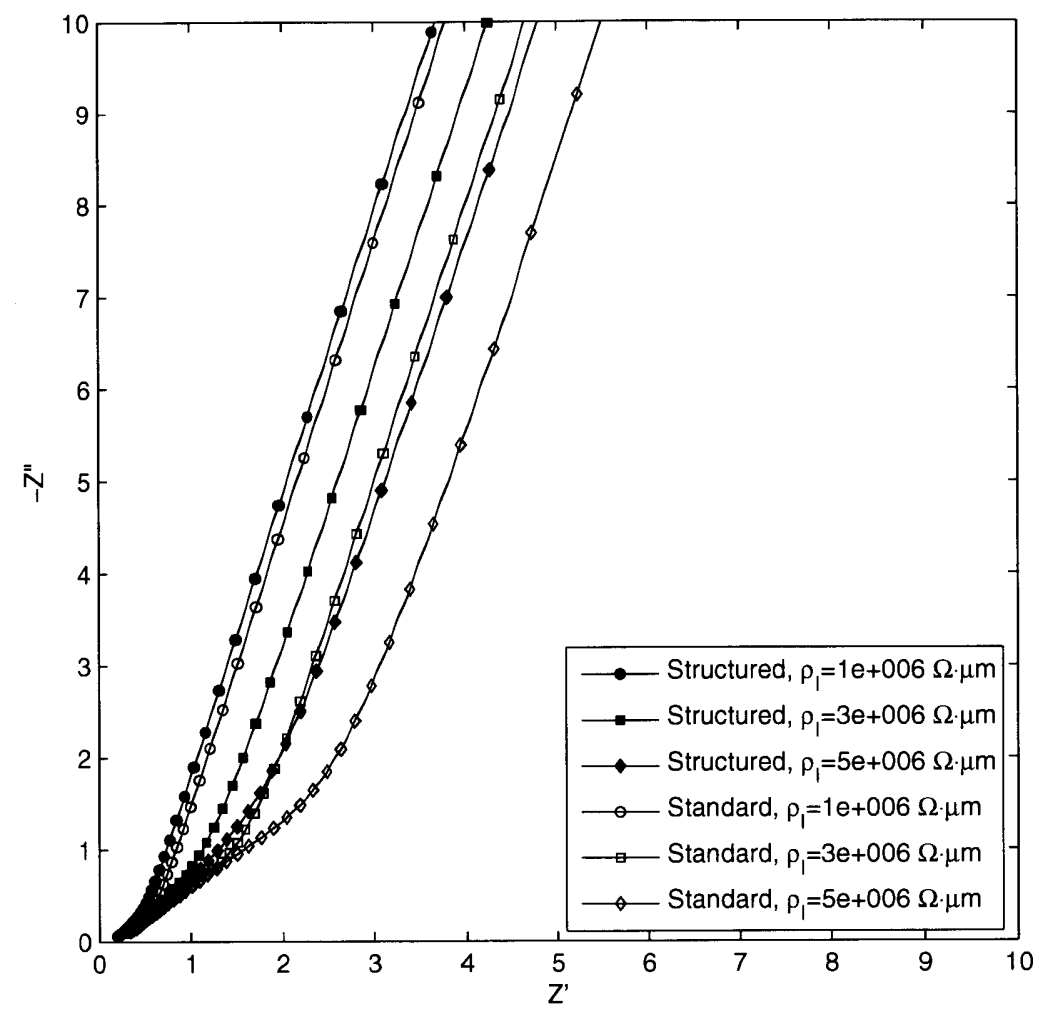

(a)

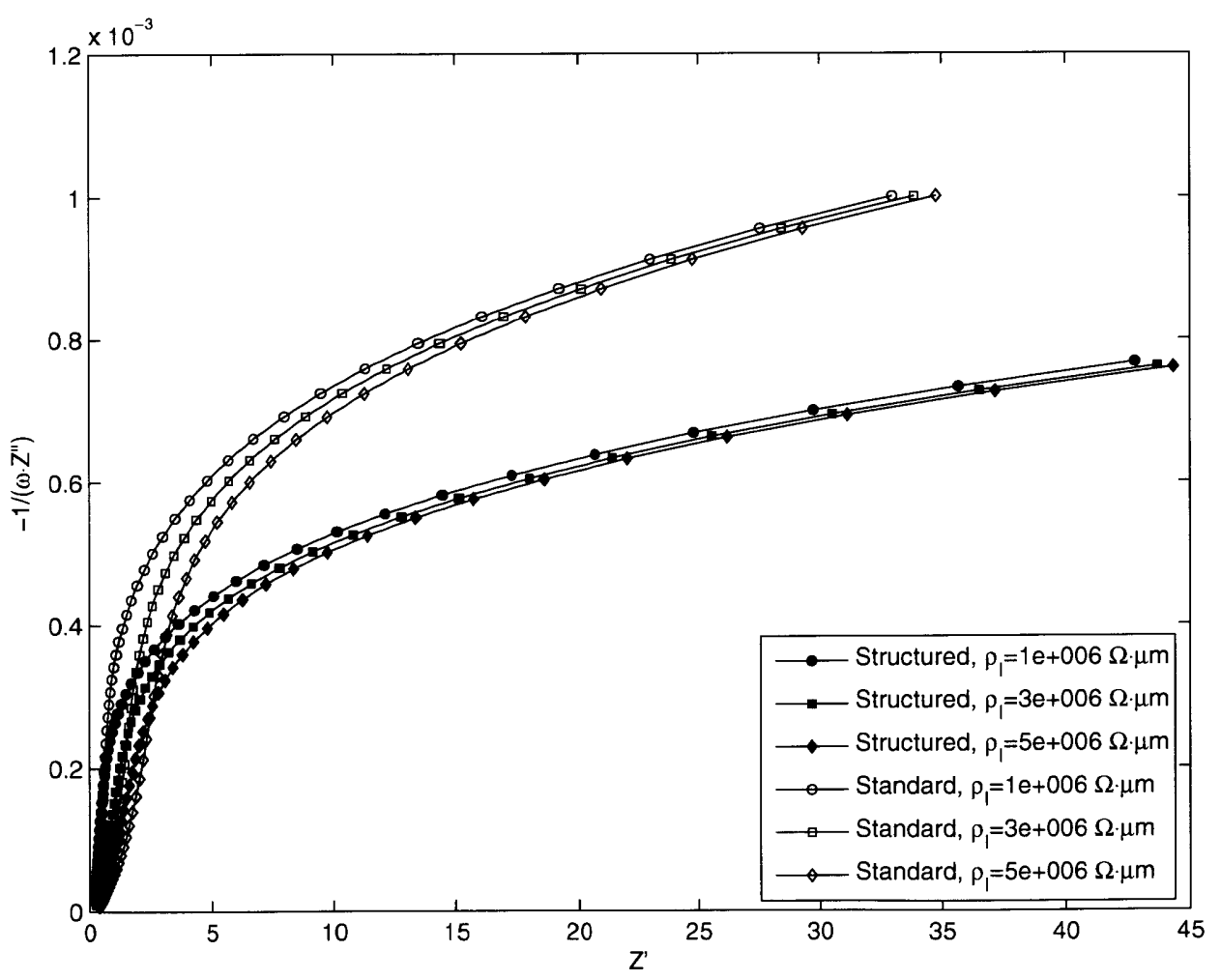

(b)

Figure 3-16: Nyquist (a) and capacitance (b) plots showing the effect that electrode ionic resistivity has on the behavior of the structured electrode model. 


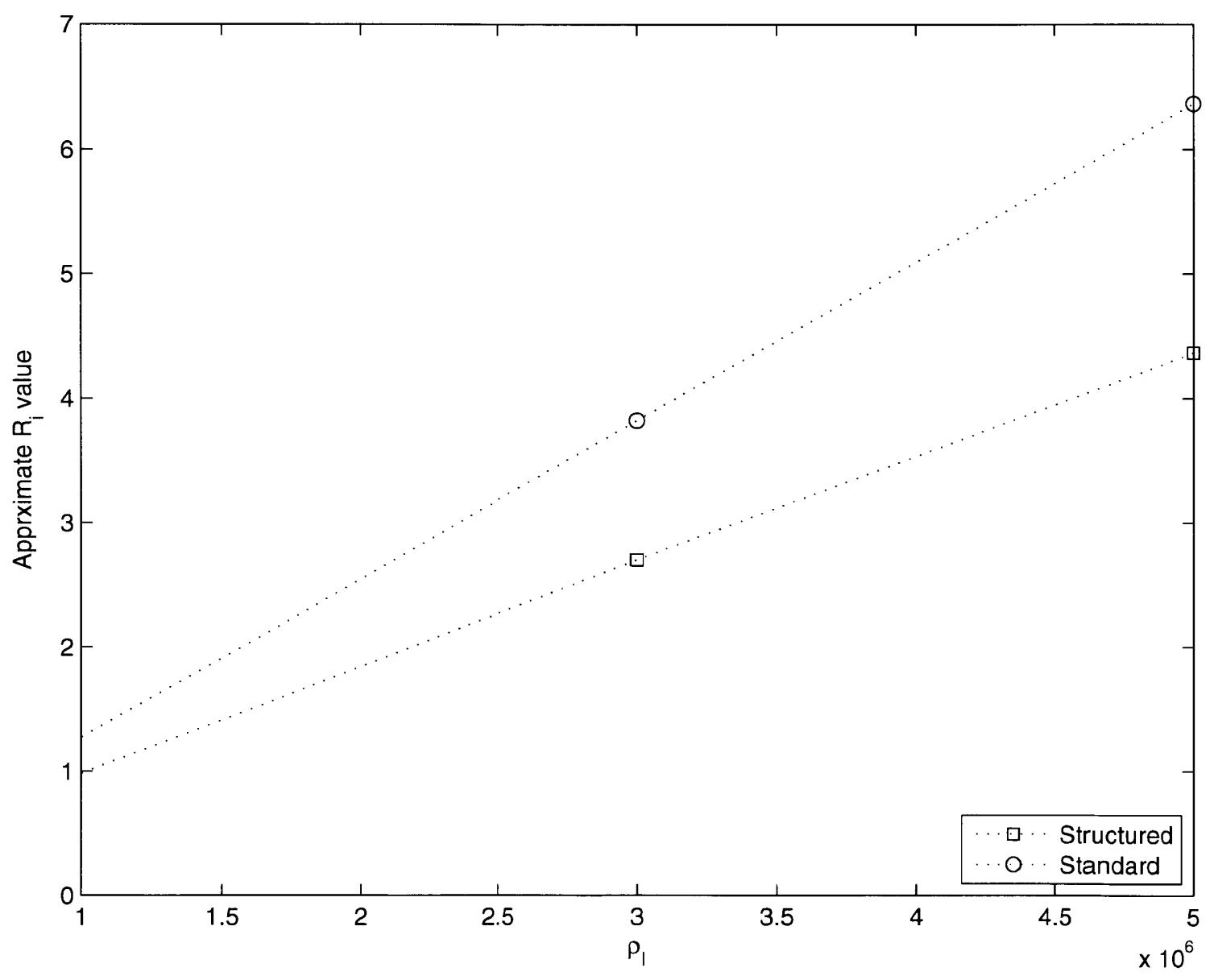

Figure 3-17: Ionic resistance value for changing electrode ionic resistivity. 


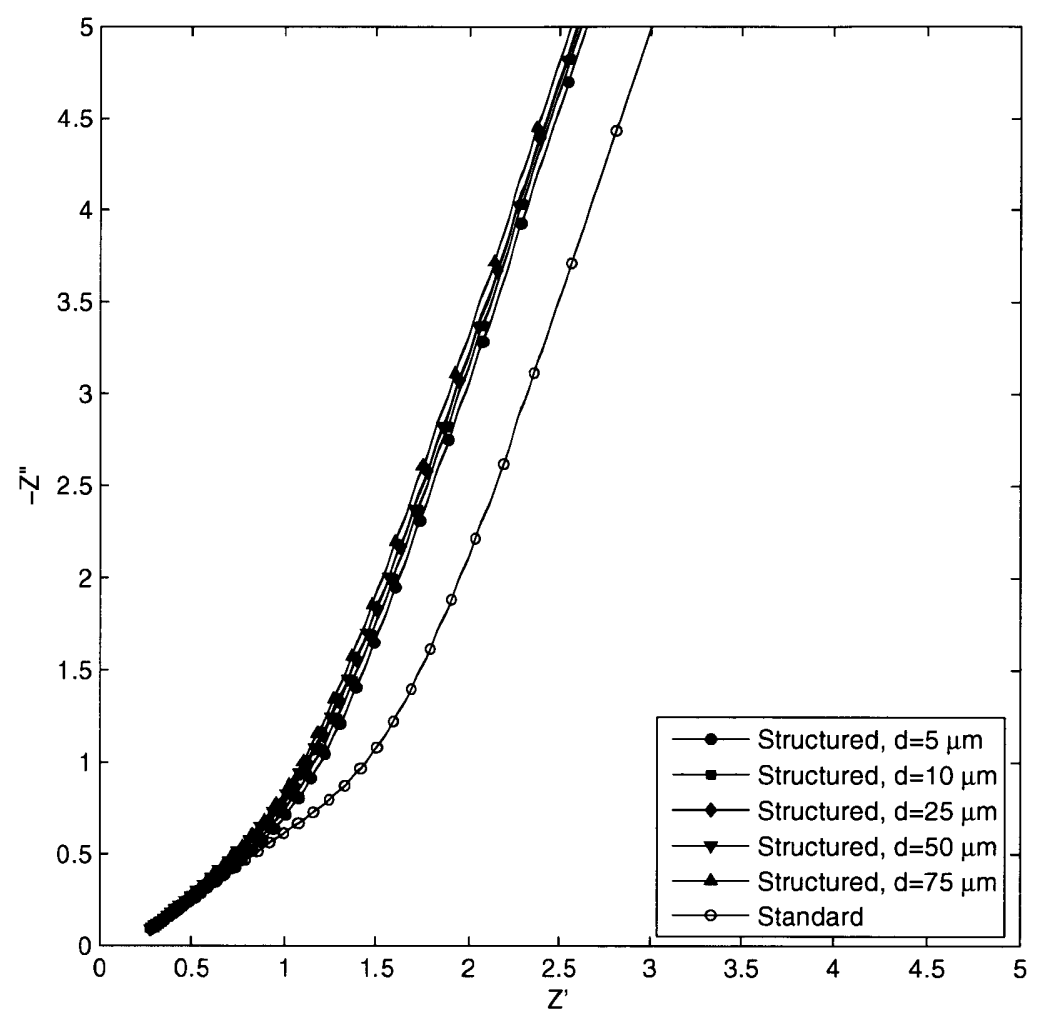

(a)

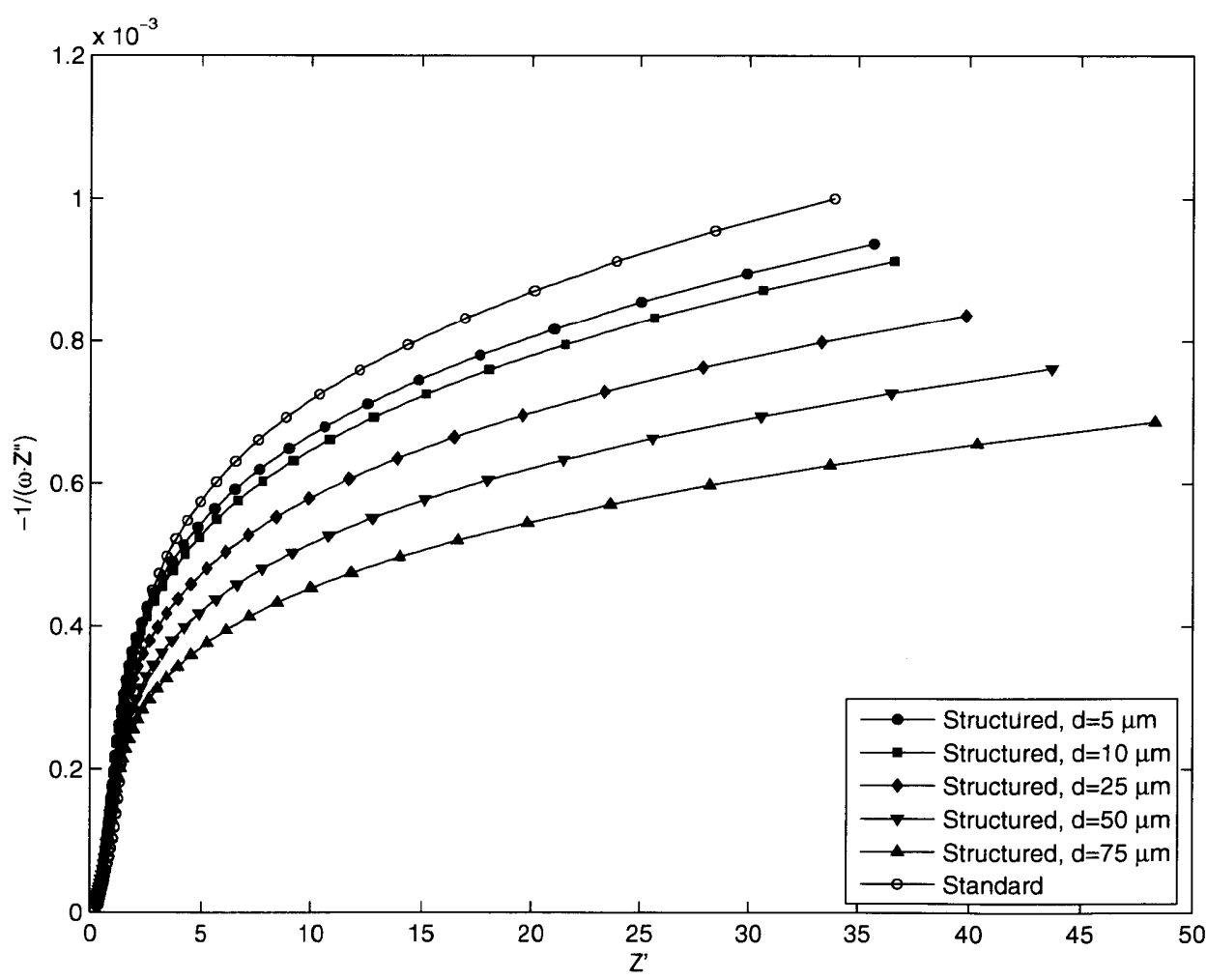

(b)

Figure 3-18: Nyquist (a) and capacitance (b) plots showing the effect that electrode ionic resistivity has on the behavior of the structured electrode model. 


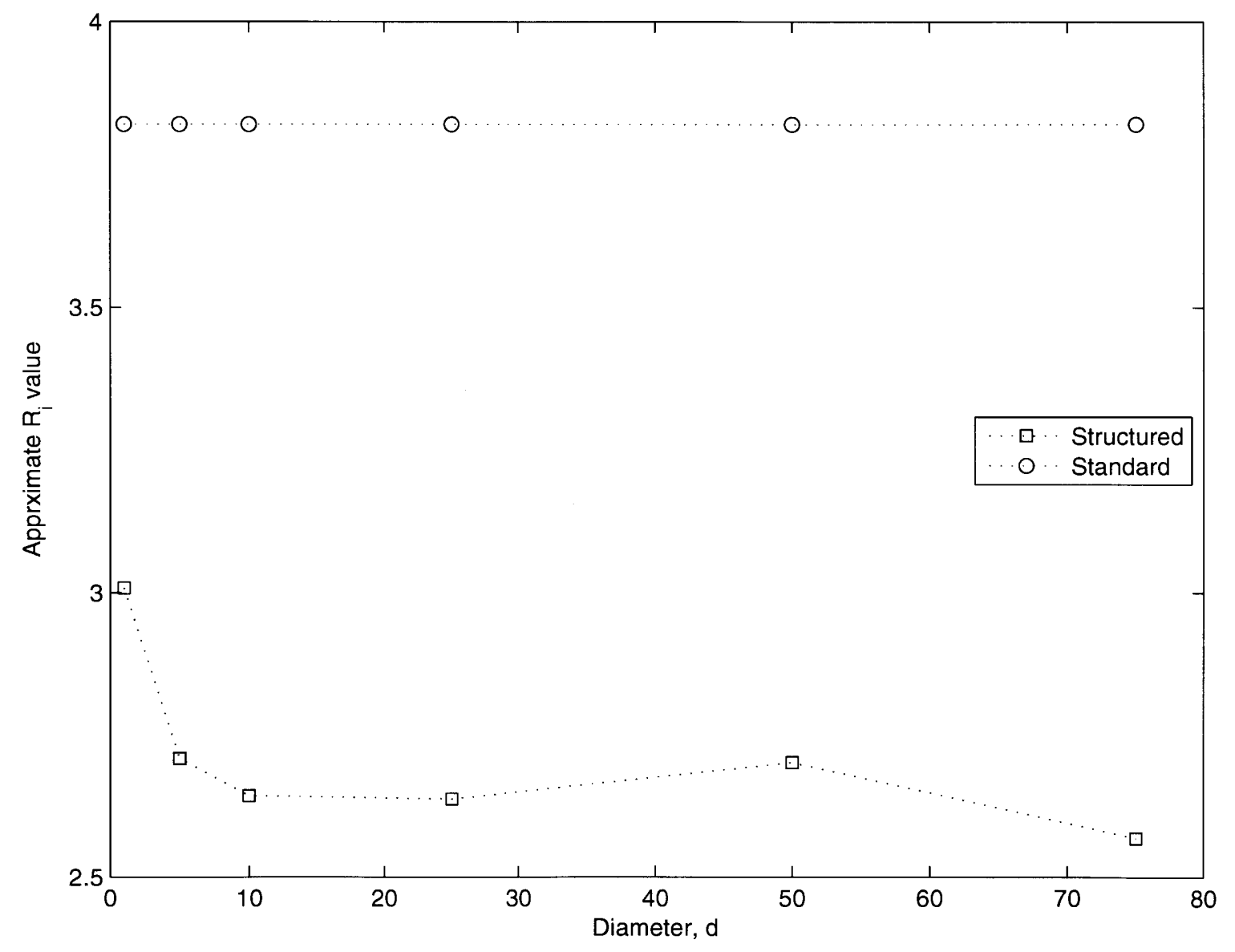

Figure 3-19: Ionic resistance value for changing structured electrode post diameters. 


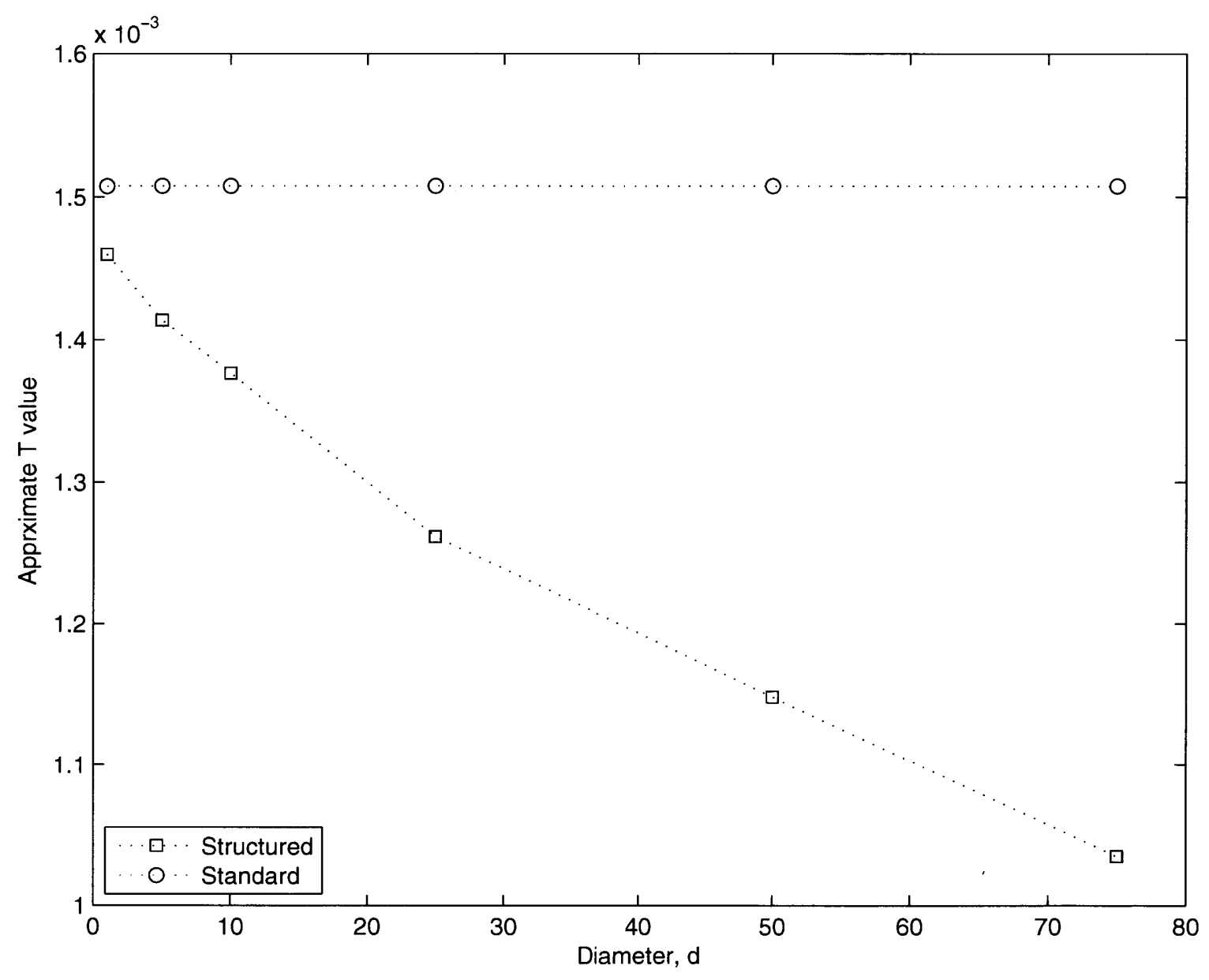

Figure 3-20: CPE capacitance value for changing structured electrode post diameters. 
in ionic resistance. A reasonable plan would be to choose a post diameter near this critical value so that there is a maximum of ionic resistance benefit with a minimum of displaced electrode material.

In the capacitance plot of these spectra, Figure $3-18 \mathrm{~b}$, the low frequency asymptote of the structured electrode is strongly dependent on the diameter of the Nafion posts. Not surprisingly, smaller diameter posts move the asymptote of the structured electrodes closer to that of the standard cell, since the small posts have less influence on the electrode behavior. The plot of the approximate capacitance value in Figure 3-20 confirms this trend.

Another crucial parameter for the structured electrode is the thickness of the electrode, which corresponds to adding more catalyst material to the electrode. Keep in mind that the thickness being controlled in this model is that of the standard electrode and the thickness of the corresponding structured cell is calculated to include the same volume of electrode material in the cell using, Equation 3.39, which leads to a larger electrode thickness. The Nyquist plot in Figure 3-21 shows the calculated impedance spectra of the model for three values of the electrode thickness. The position of the low-frequency asymptotes indicates that the ionic resistance of the structured electrode is much lower than that of the conventional electrode at high electrode thicknesses, yet there is little difference in ionic resistance at lower electrode thicknesses. Figure 3-22 illustrates this point by plotting the approximate ionic resistance values as a function of electrode height. This clearly shows that there is a minimum thickness below which adding Nafion posts does not improve ionic conductivity. Also visible in this plot is the non-linearity of ionic resistance in a structured electrode. In the conventional electrode, ionic resistance is linear with electrode thickness. In the structured electrode, however, ionic resistance seems to level off as electrode thickness becomes large, indicating that the larger the electrode thickness, the more useful ionically conductive channels will become.

The final geometric parameter of interest in the structured electrode model is the post spacing. The impedance spectra for several post spacings are shown in Figure 3-23. The Nyquist plot in Figure 3-23a clearly shows that the ionic resistance of the structured electrode is lower for closer post spacing. However, as with the post diameter, even very large post spacing offers significant ionic resistance improvements over the standard electrode. The capacitance plot of these spectra, shown in Figure 3-23b, shows a similar result to that found with post diameter variations. As the post spacing increases, the electrode behaves 


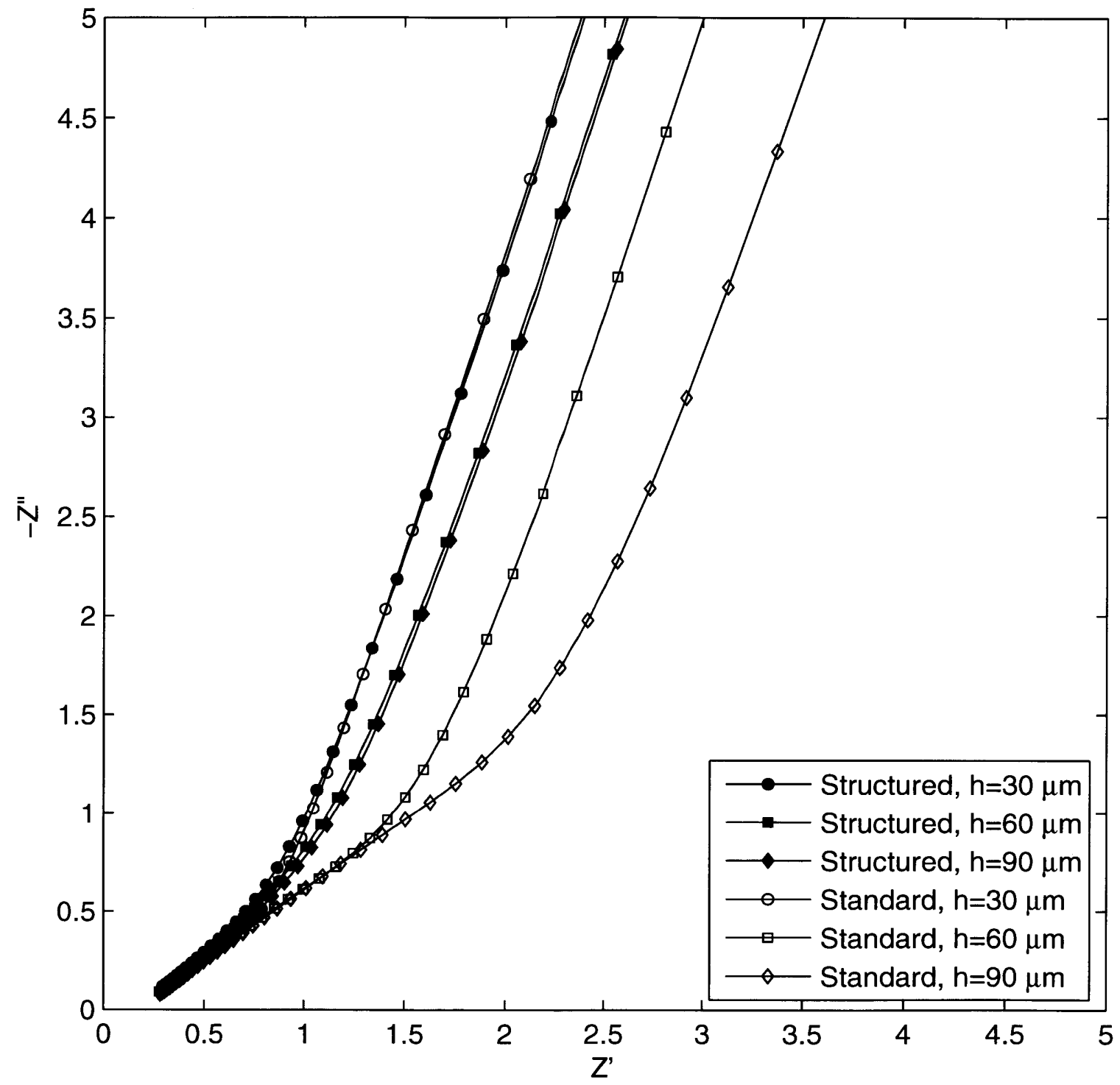

Figure 3-21: Nyquist plots showing the effect that electrode thickness has on the behavior of the structured electrode model. 


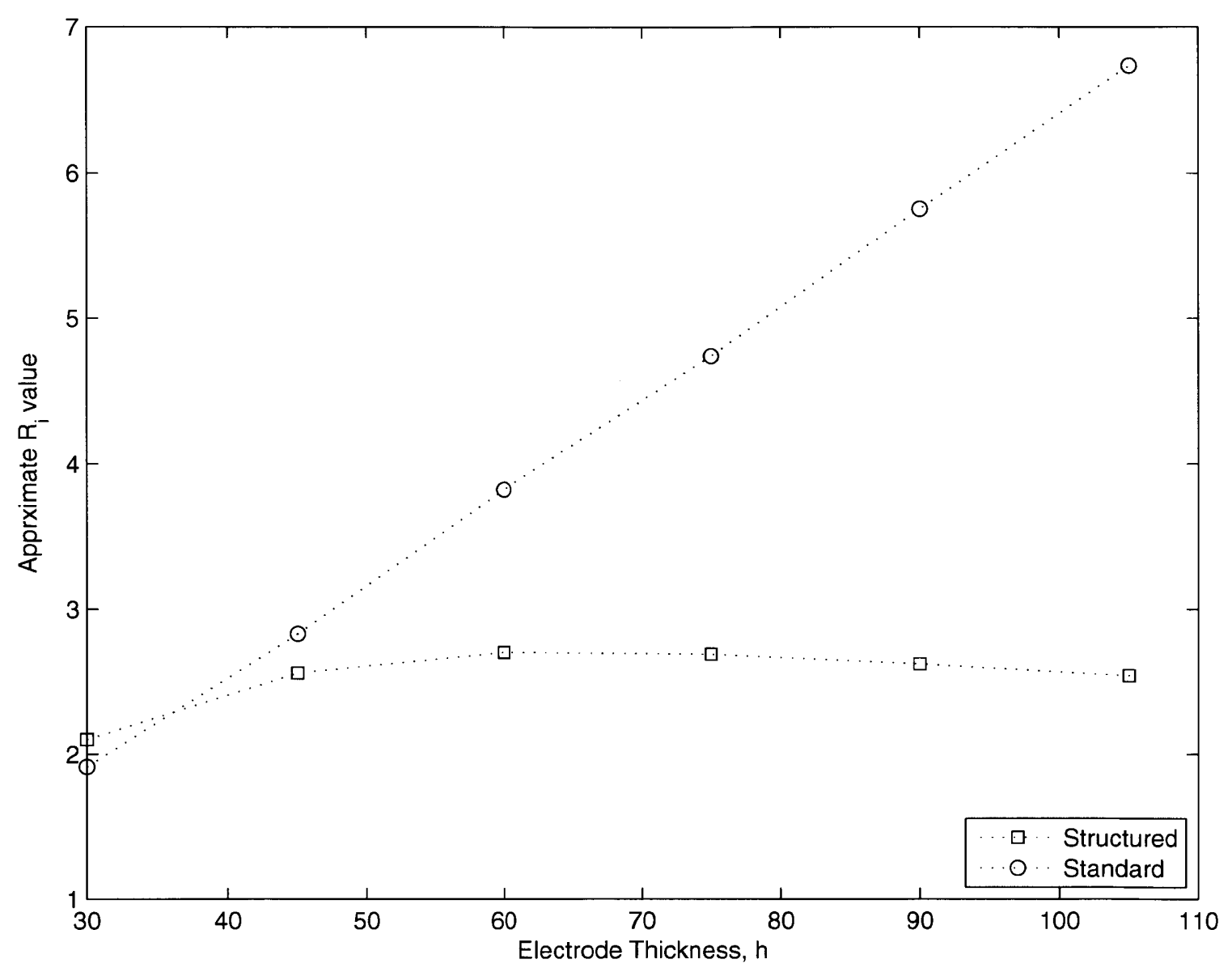

Figure 3-22: Ionic resistance value for changing electrode thickness. 


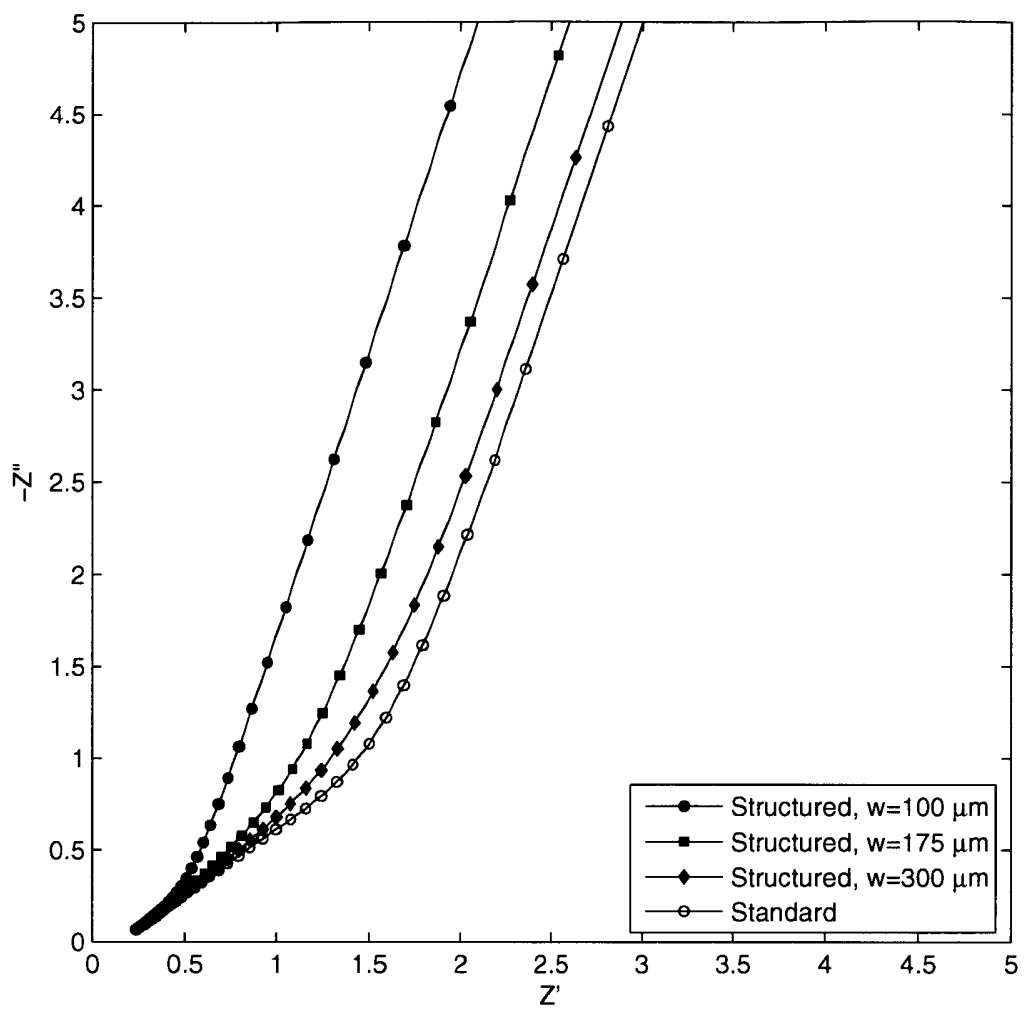

(a)

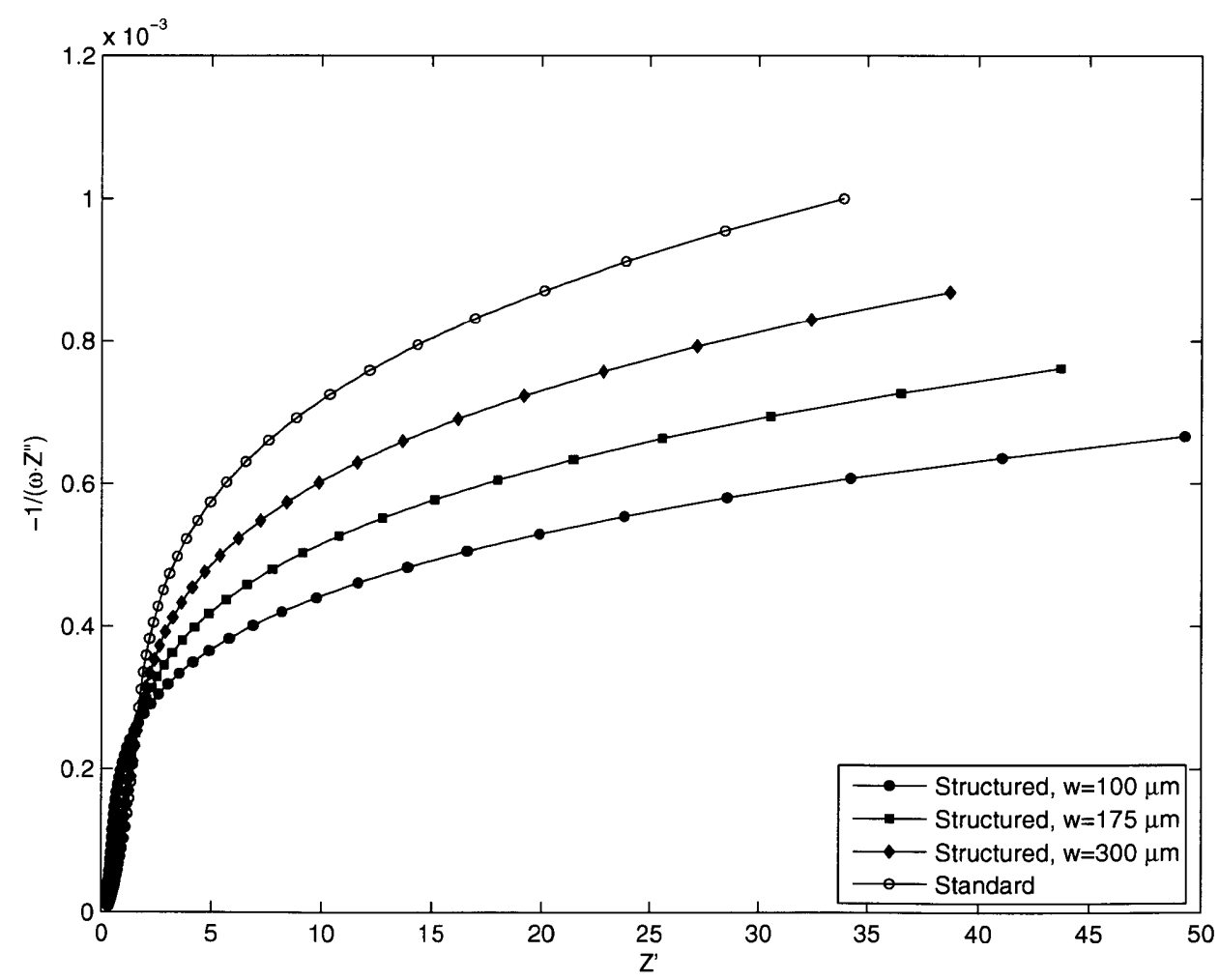

(b)

Figure 3-23: Nyquist (a) and capacitance (b) plots showing the effect that Nafion post spacing has on the behavior of the structured electrode model. 
more like a standard electrode, which means an increase in the capacitance value of the low-frequency asymptote.

Another way to look at the influence of structured electrode geometry is to consider a few dimensionless geometry parameters, such as the ratio of the post diameter to post spacing and the aspect ratio of the gap between posts. Figure 3-24 shows the Nyquist and capacitance plots obtained at several post diameters while keeping the ratio of post diameter to post spacing at 0.5. As seen in the Nyquist plot in Figure 3-24a, the electrodes with larger diameter posts do have slightly larger ionic resistance values, but the change with post diameter is relatively small. In the capacitance plot, shown in Figure 3-24b, the change with post diameter appears even less significant.

Plotting the approximate ionic resistance term, shown in Figure 3-25, and CPE capacitance term, shown in Figure 3-26, as a function of $d / w$ helps to illustrate the behavior of the structured cells. For large posts, the ionic resistance increases significantly as post density decreases. For smaller posts, the increase in ionic conductivity is much less significant as post density decreases. The effect of post density on CPE capacitance seems to be almost unchanged with post diameter. For all post diameters, the CPE capacitance increases with decreasing post density so that the capacitance value approaches that of the standard electrode as posts become sparse.

The other dimensionless parameter describing the structured electrode is the aspect ratio of the gap between neighboring posts. This gap aspect ratio is:

$$
A R_{g a p}=\frac{h}{w-d}
$$

The ionic resistance values and CPE capacitance values are plotted as a function of gap aspect ratio in Figures 3-27 and 3-28, respectively. The ionic resistance for the structured cell depends strongly on the gap aspect ratio, but is largely invariant to post diameter when the gap aspect ratio is kept constant. For low gap aspect ratios, below 2:1, the ionic resistance changes quickly with changes in aspect ratio. Beyond aspect ratios of $2: 1$, however, the ionic resistance does not change much at all. The capacitance value, on the other hand, is clearly not determined solely by the gap aspect ratio. While small aspect ratios lead to behavior more similar to the standard electrode, changes in post diameter also significantly change the capacitance values. 


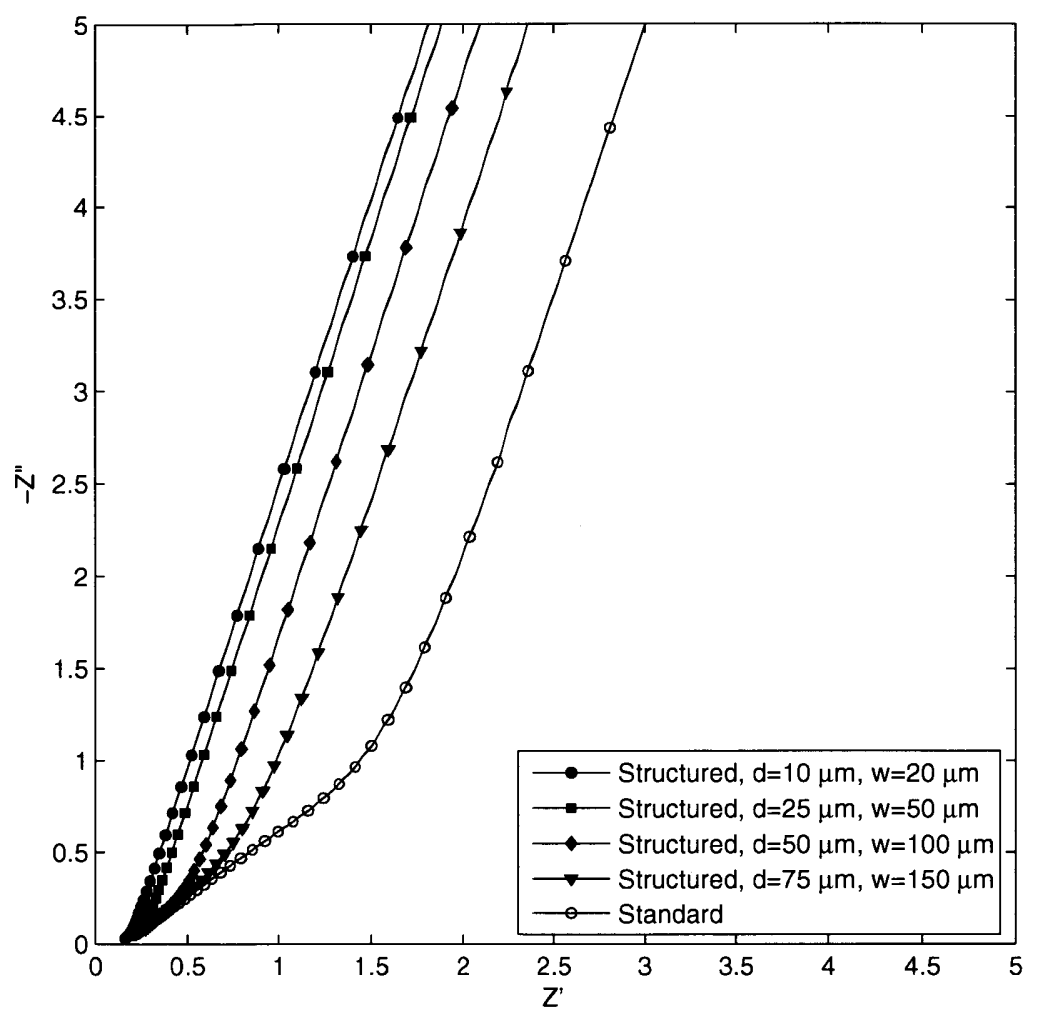

(a)

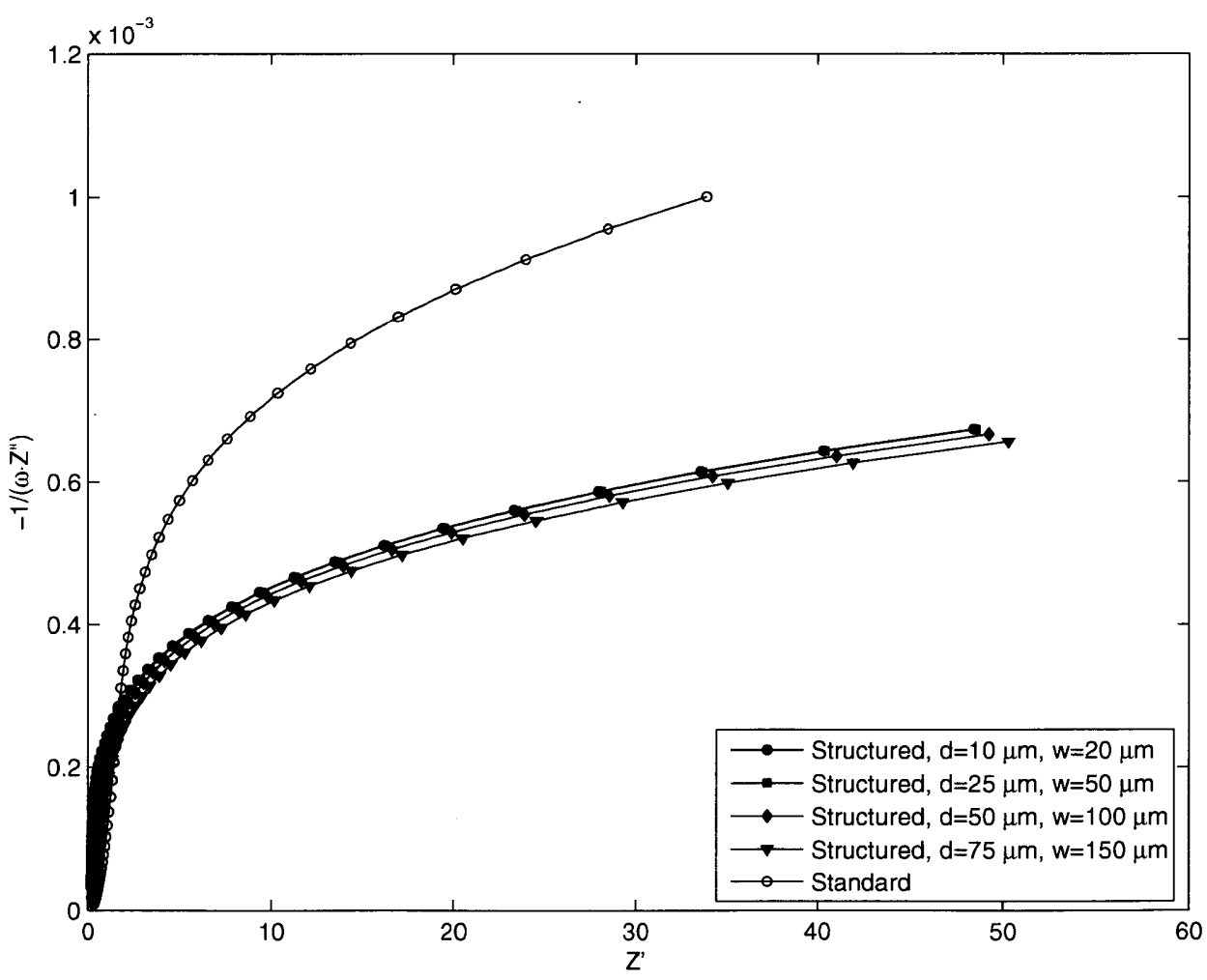

(b)

Figure 3-24: Nyquist (a) and capacitance (b) plots showing the effect of changing the Nafion post diameter while keeping the ratio of post diameter to post spacing constant. 


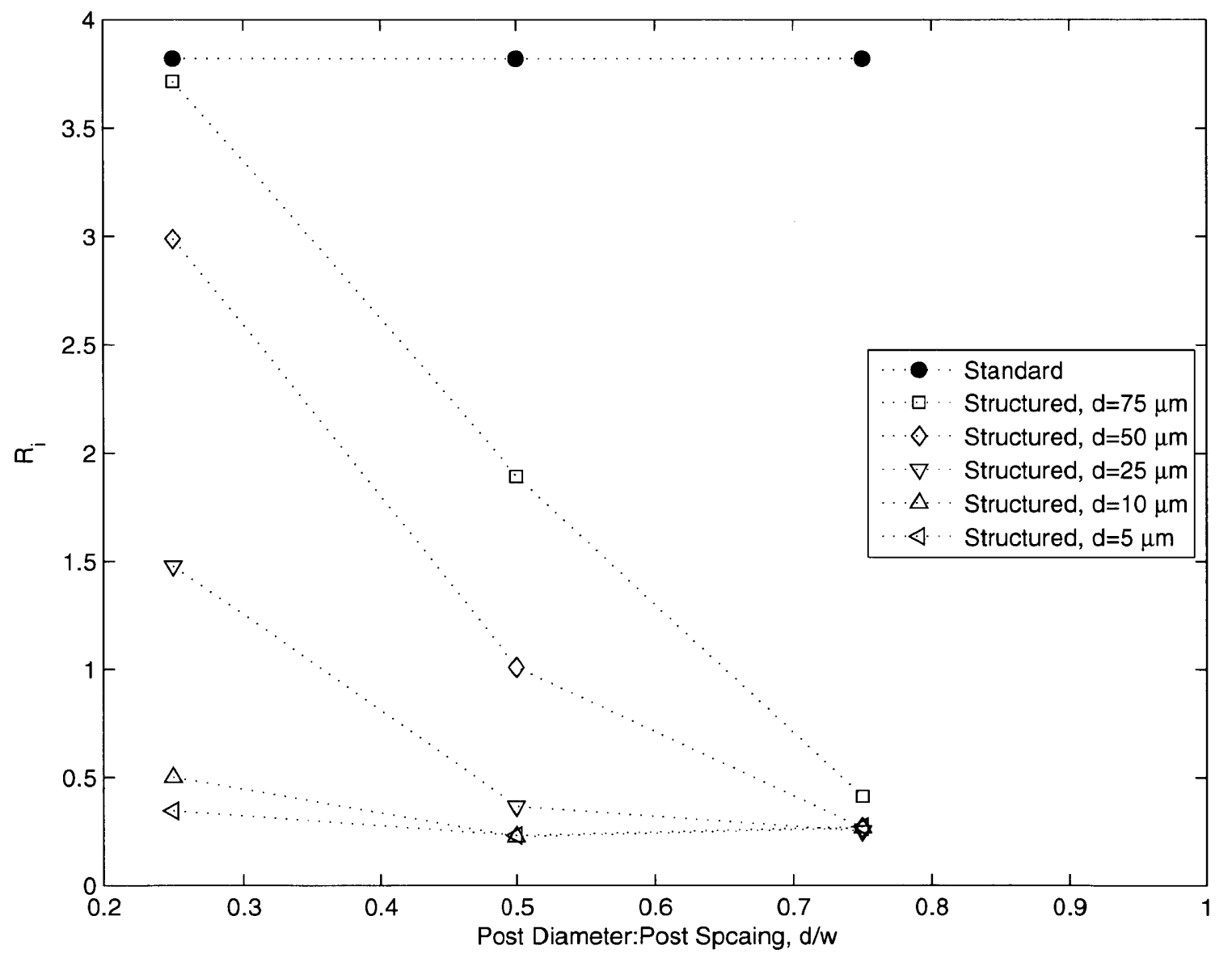

Figure 3-25: Effect of post spacing ratio, $d / w$, on ionic resistance. 


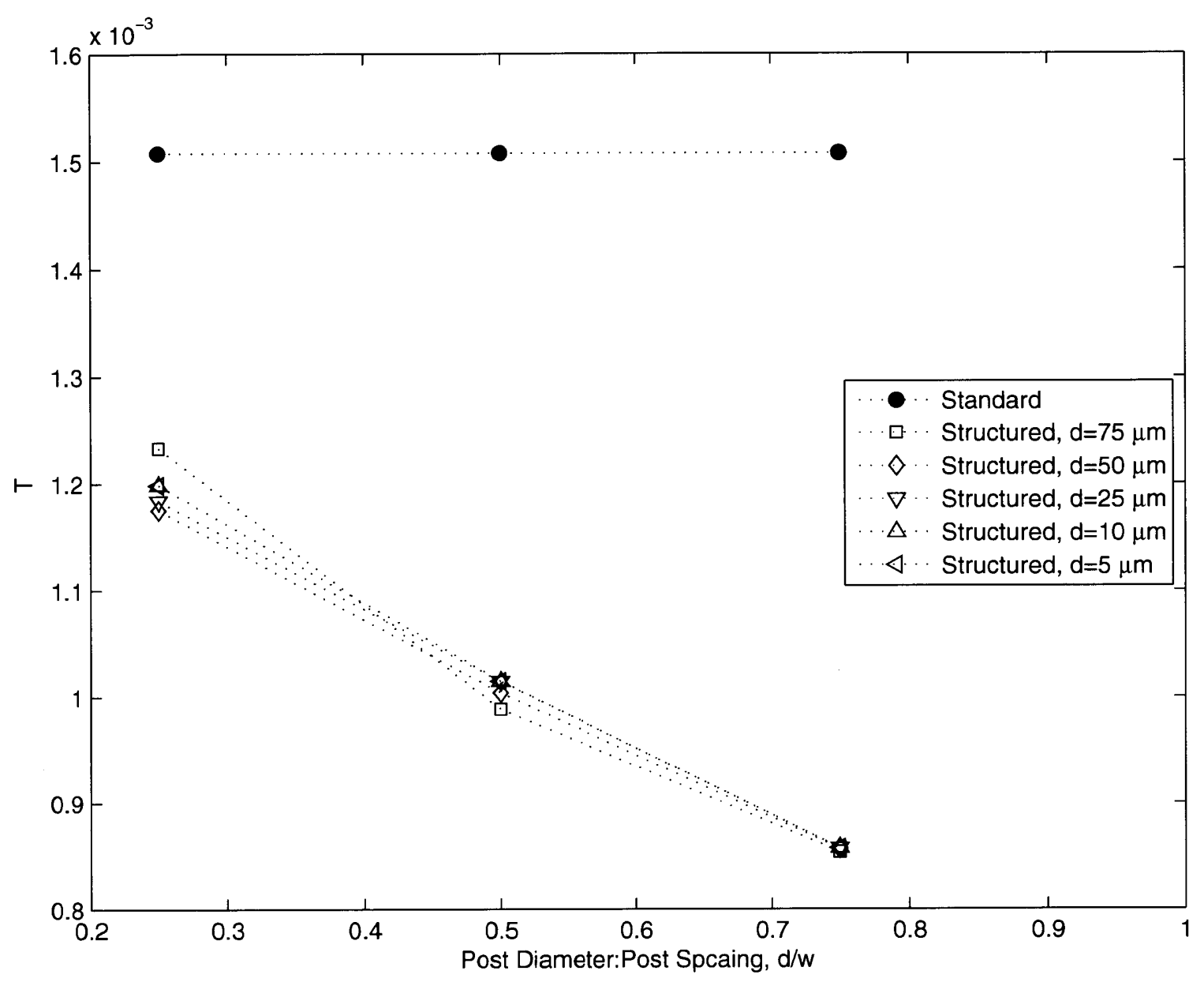

Figure 3-26: Effect of post spacing ratio, $d / w$, on capacitance. 


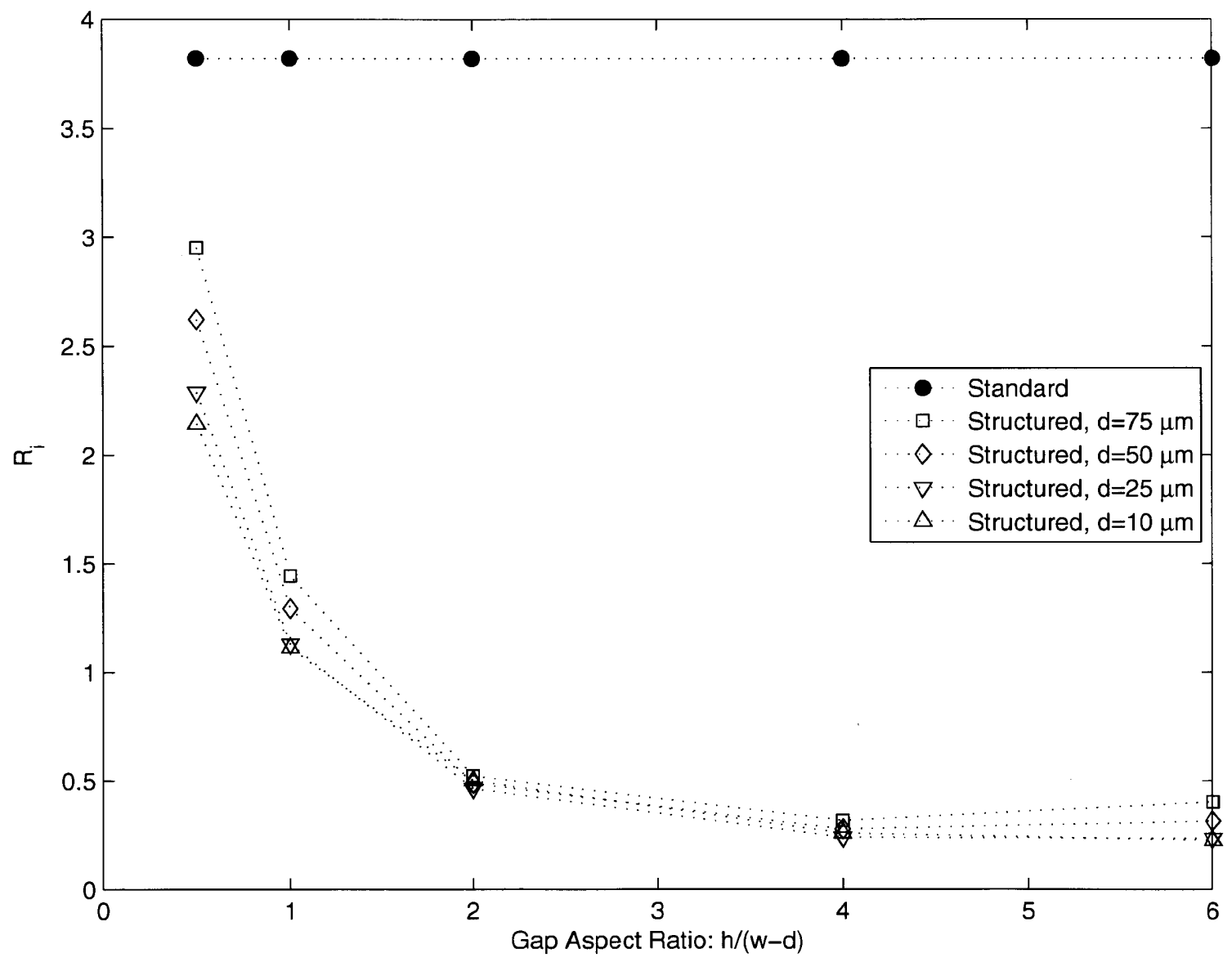

Figure 3-27: Effect of gap aspect ratio on ionic resistance. 


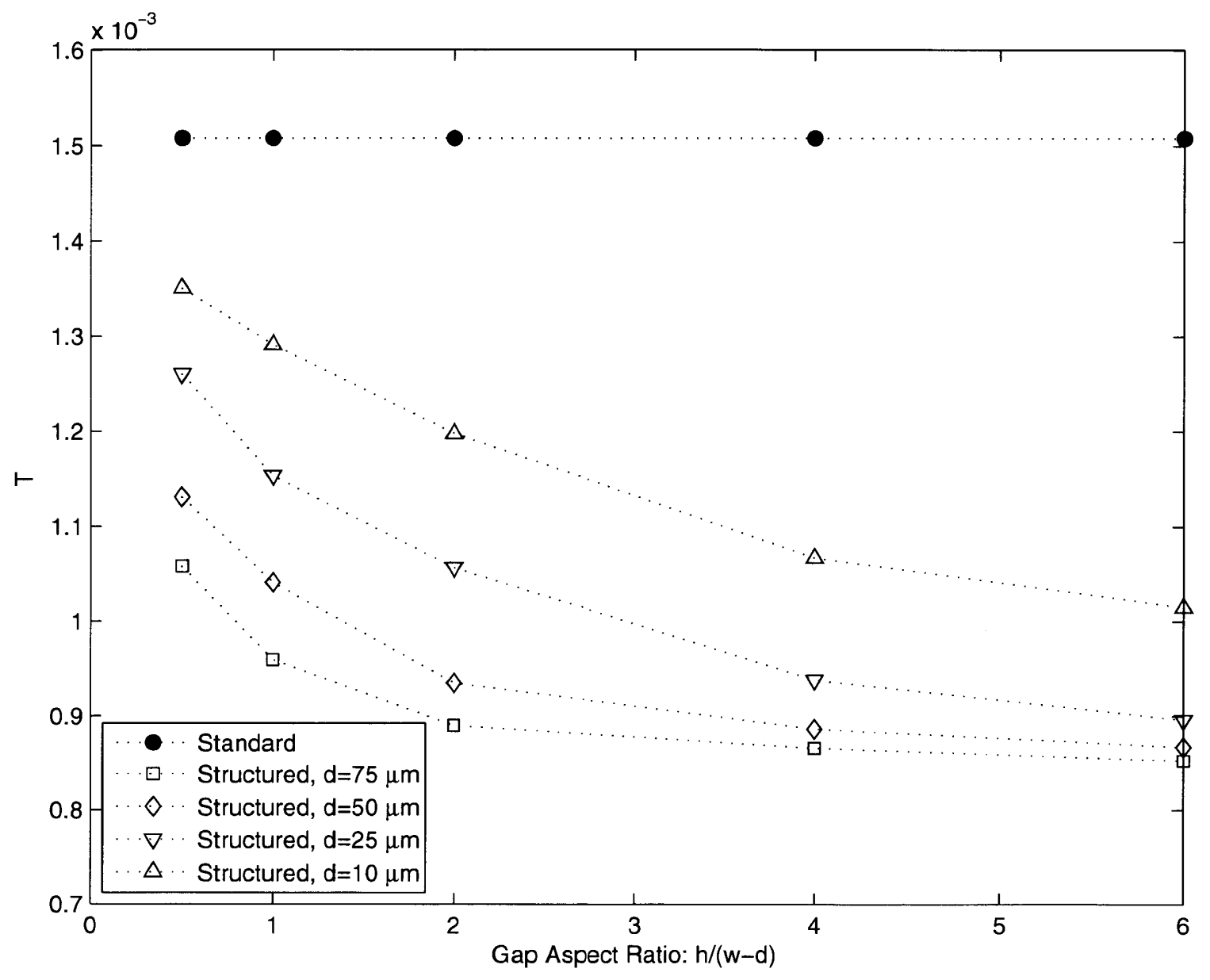

Figure 3-28: Effect of gap aspect ratio on capacitance. 


\subsubsection{Utility of the Two-Dimensional Structured Model}

The two-dimensional structured model was formulated to estimate the effect of electrode geometry on the ionic resistance of structured electrodes. Based on the model, the general predictions regarding ionic resistance in the electrode were confirmed. Nafion posts do indeed reduce ionic resistance in the electrode and the posts become vastly more effective in electrodes with initially low ionic conductivity. Similarly, the prediction that the aspect ratio of the gap between posts is crucial in determining the effectiveness of structured electrode, illustrated in Figure 3-1, is confirmed by the simulation results plotted in Figure 3-27, which shows ionic resistance is strongly dependent on the gap aspect ratio.

The two-dimensional model does provide one curious and unexpected result. The structured electrode does not improve an electrode's effective capacitance. As discussed in relation to the transmission line model, the two-dimensional electrode model also cannot predict the loss of electrode effectiveness observed when there is too much catalyst added to an electrode. Since the two-dimensional model is directly analogous to the transmission line model, this in itself is not surprising. However, it would still be reasonable to expect that the geometry of the two-dimensional model could show an improvement in capacitance. That the two-dimensional model does not show this behavior is further indication that the underlying mechanism that leads to the catalyst loading limit is not captured in the simplified electrode models presented in this thesis.

The three models considered in this chapter, the electrical network model, the transmission line model, and the two-dimensional structured model, are all based on the same simplifications of electrode behavior that do not consider many effects present in operating cells. As a consequence, they cannot be used to predict electrode behavior directly. They do, however, offer insight into the nature of the design problem as it relates to ionic conductivity in the electrode. Based on the two-dimensional structured model, electrode geometry is selected that will result in higher ionic conductivity in the structured electrode as compared to a conventional electrode. The influence of this design change must be studied experimentally to determine the influence of ionic resistivity on DMFC anode performance. 


\subsection{Summary}

Axiomatic Design analysis of the standard DMFC anode indicates that performance limitations on the anode are a result of poor design strategy employed in the development of the standard design. In particular, the method used to ensure ionic conductivity through the electrode leads to many undesirable side effects, including blocking fuel and carbon dioxide transport in the anode. The structured anode design with Nafion posts through the thickness of the anode is proposed as a design solution that will eliminate all the unintended consequences of controlling ionic conductivity with the amount of Nafion dispersed in the anode.

The importance of ensuring effective ionic conduction throughout the electrode is reinforced with the analysis of the transmission line model for the DMFC anode using several different charge transfer elements. The transmission line models show the large disparity

in reaction rate between the catalyst particles near the membrane and those far from the membrane. In conventional electrodes, it turns out that the vast majority of the current is transferred in the third of the electrode nearest the membrane. This results in large anodic overpotentials and decreased efficiency. If the ionic resistance can be reduced, the reaction current can be spread equally throughout the anode and results in a large drop in anodic overpotential. The transmission line model can also be used to predict the impedance spectra of electrodes. The model illustrates that the ionic resistance in the electrode can be easily extracted from Nyquist plots of impedance spectra by considering the position of the low-frequency asymptote.

A two-dimensional model of the structured electrode allows for the impact of the structured electrode on the anode design matrix to be evaluated. First, the model shows that the addition of posts greatly reduces ionic resistance in the anode. Additionally, the ionic resistance of the anode can be controlled by changing the geometry of the posts. In particular, the gap aspect ratio of the posts has a strong influence on electrode ionic resistance. Figure 3-22 clearly shows that catalyst loading has little effect on ionic resistance in the structured electrode. This result supports the removal of the coupling term between $F R_{1}$ and $F R_{5}$ in the design matrix of the structured electrode in Table 3.3. All modeling results indicate that the structured electrode should function as intended, but the actual performance of the structured electrodes must be verified by experiment. The methods to evaluate 
the structured electrode are described in Chapter 4 and the results of those experiments are presented in Chapter 5. 


\section{Chapter 4}

\section{Experimental Methods and Procedures}

The electrodes produced in this thesis were evaluated using two popular electrochemical techniques: Cyclic Voltammetry and Electrochemical Impedance Spectroscopy. This chapter presents the basic characteristics of both techniques along with the particular experimental conditions used to gather the data presented in this thesis. Consult any electrochemistry textbook [8] for a complete description of the theory behind either technique. This chapter also describes the fabrication procedures used to create all of the electrodes used in this thesis.

\subsection{Cyclic Voltammetry}

Cyclic voltammetry is a common electrochemical measurement method in which the potential of an electrode is swept back and forth over a range of potentials at a fixed rate. This allows information to be gathered about how both time and potential affect the system from one experiment. Additionally, the repeated scans between potential limits means that the electrode surface undergoes a repeatable preparation before each reversal of scan direction. This means that cyclic voltammetry can allow the study of electrode surfaces with very repeatable initial surface conditions from scan to scan [34]. Cyclic voltammetry is typically used as an early diagnostic tool before using more specialized methods to focus in on a behavior of interest [8].

All cyclic voltammetry experiments in this thesis were performed using the three electrode- 
cell described in Section 4.3.1. The electrolyte used was $0.5 \mathrm{M}$ sulfuric acid under a nitrogen atmosphere unless otherwise stated. The working electrodes were mounted on a polished $4 \mathrm{~mm}$ diameter glassy carbon support except for the platinum disc which was directly mounted in a Teflon sheath. The counter electrode was a piece of platinum mesh. All potential measurements were taken against a silver-silver chloride $(\mathrm{Ag} / \mathrm{AgCl})$ reference electrode.

The cyclic voltammetry measurements were performed using a custom-made potentiostat with data acquisition and control achieved using a National Instruments USB-6211 DAQ with Labview software. Each Cyclic Voltammetry experiment began with several sweeps over the range of interest to eliminate any initial transient sweeps. The initial sweep was performed at $100 \mathrm{mV} / \mathrm{s}$ from $-0.25 \mathrm{~V}$ to $1.25 \mathrm{~V}$ vs the $\mathrm{Ag} / \mathrm{AgCl}$ reference electrode. Once it appeared that a steady-state had been reached, the actual data-gathering sweeps were run. These were performed at a slower rate of $50 \mathrm{mV} / \mathrm{s}$ and the range was selected such that the electrolysis of water did not dominate the current measurement at the ends of the sweep. This typically meant a potential sweep from about $-0.2 \mathrm{~V}$ to $1.2 \mathrm{~V}$ vs $\mathrm{Ag} / \mathrm{AgCl}$ reference. Again several data sets were taken to make sure that the system had reached a steady state. Each data set consisted of about 5 sweeps over the range of interest.

\subsubsection{Electrochemically Active Area}

One powerful and popular use for cyclic voltammetry is the estimation of active catalyst surface area in an electrode. The technique uses the hydrogen adsorption peaks to determine active platinum area [35]. The idea is to find the total electrochemical charge on the platinum surface and relate that to area of the platinum surface. It has been determined that hydrogen, adsorbed on polycrystalline platinum in a mono-layer, coats the platinum with a density of $210 \mathrm{C} / \mathrm{cm}^{2}$. The total charge due to hydrogen adsorption on a surface is the integral of the current carried by hydrogen to the surface over the period of charging. The current measured in cyclic voltammetry includes the hydrogen adsorption current that varies with potential, a constant double-layer charging current, and a current due to the electrolysis of water in

the electrolyte. The double-layer current is constant and is easily subtracted from the total current. It can be found from the constant current section in the downward potential sweep just before hydrogen adsorption begins, as shown in Figure 4-1. Removing the current due to electrolyte breakdown is more challenging, since it is not constant. If there were 
no electrolyte breakdown and the potential sweep continued in the negative direction, the total current should return to the constant double-layer charging current after the hydrogen adsorption region was passed. In this case, simply integrating the current, from the point where it diverges from the constant double-layer current value, to the point where the current returns to the constant double-layer current value, would give the total charge of hydrogen adsorbed on the surface. Unfortunately, the aqueous electrolyte breaks down just after peak of the hydrogen adsorption current and the potential sweep must switch directions before the hydrogen adsorption current can decay to zero. It has been determined that choosing the limit of the current integration to be at the minimum current point, just after the last hydrogen adsorption peak, gives an accurate measure of total hydrogen adsorption charge. At this point, increasing current due to the electrolysis of the electrolyte within the integration limits is assumed to roughly offset the hydrogen adsorption current that is decaying beyond the limit.

Figure 4-1 shows the area of integration, in gray, used to determine the total hydrogen charge adsorbed onto a platinum disc. Using this method, the active platinum area of the disc was found to be $0.161 \mathrm{~cm}^{2}$ and the measured physical area of the disc was $0.146 \mathrm{~cm}^{2}$. The electrochemically active area should be slightly larger than the measured physical area, because the surface has not been polished and any roughness will add active area. This experiment was performed in order to confirm the viability of this method for determining active area and the agreement between the two areas suggests that this method is sufficient.

\subsubsection{Electrode Ionic Resistance}

In addition to measuring available catalyst area, cyclic voltammetry can be used to qualitatively measure the ionic resistance in an electrode. To illustrate this, a simple cyclic voltammetry experiment was performed on a standard electrode. The electrode was allowed to dry, then a small amount of $5 \%$ Nafion solution was applied to the electrode. Just enough Nafion was used to cover the whole electrode area and the electrode was allowed to dry with the new impregnated layer of Nafion. A new cyclic voltagram was measured and compared to that of the original electrode. The result of this experiment is shown in Figure 4-2. The original electrode shows very well defined hydrogen adsorption peaks and has an active catalyst area of $12 \mathrm{~cm}^{2}$. After the electrode has been covered in Nafion, the peaks are much lower and skewed in the direction of the potential sweep, as would happen with additional 


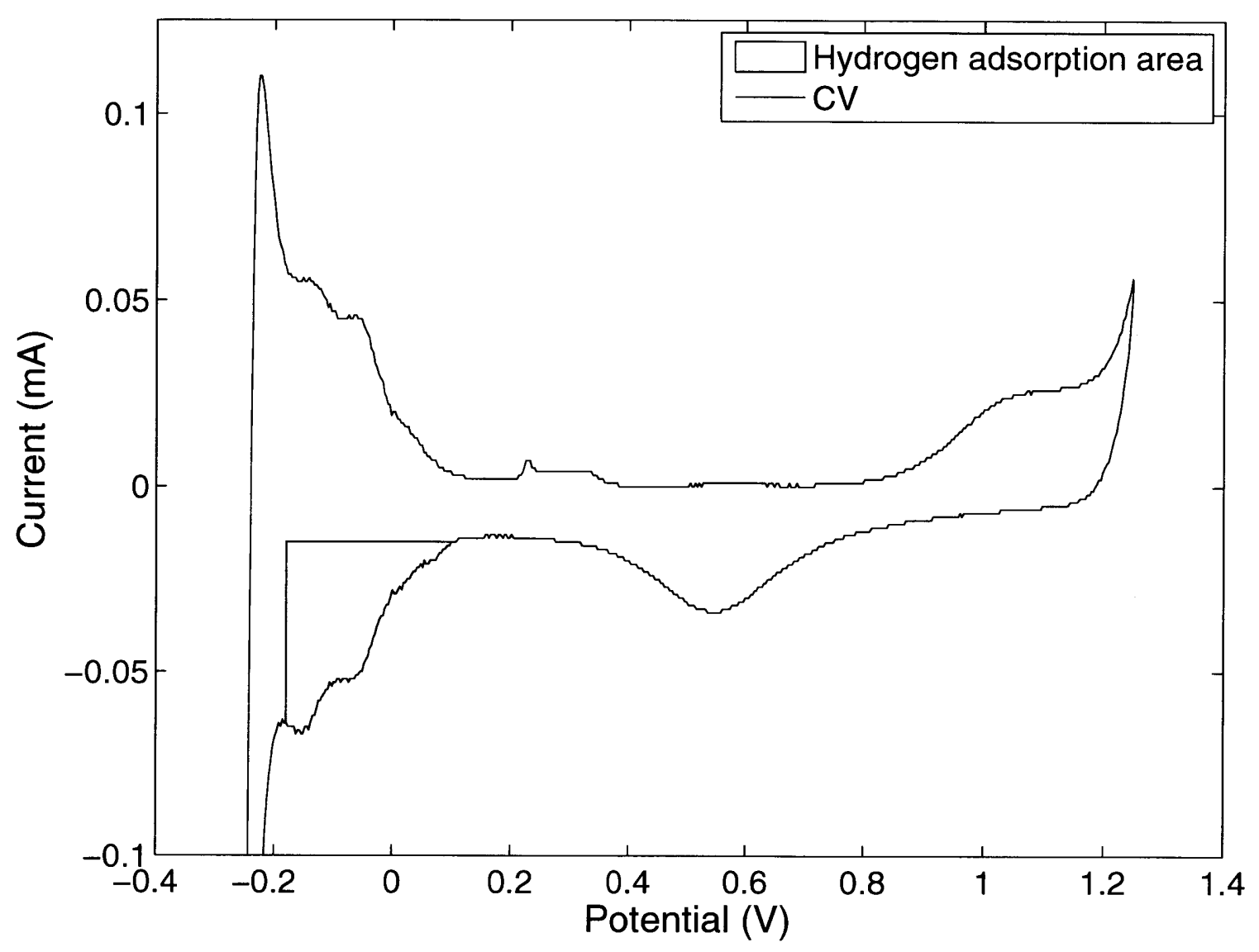

Figure 4-1: A cyclic voltagram of a platinum disc illustrating the use of hydrogen adsorption area to measure the active platinum area using the adsorbed hydrogen method. The area between the voltagram and the constant double-layer charging current, shown in red, is proportional to the electrochemically active area of platinum. 


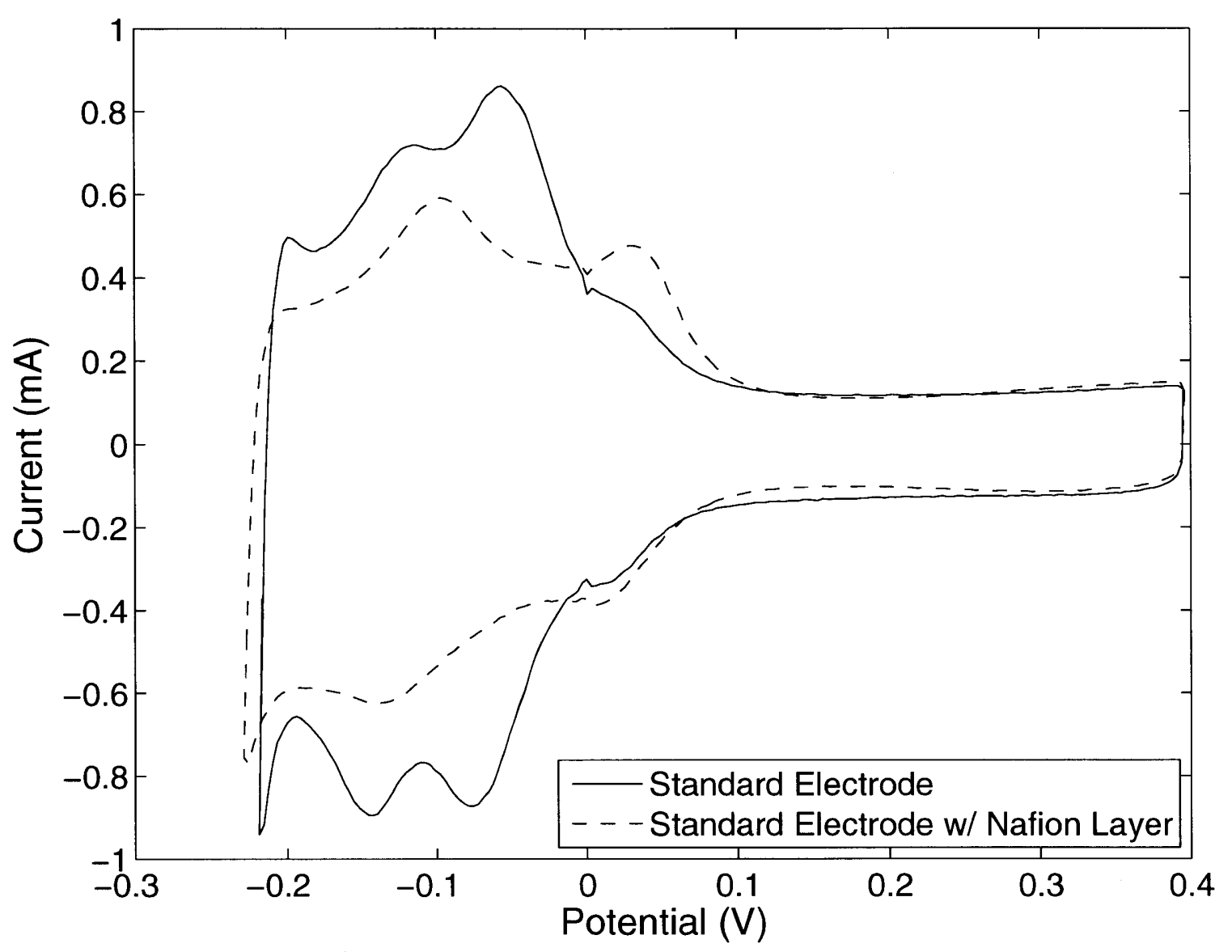

Figure 4-2: Voltagram of standard electrode and an identical electrode encased within a layer of Nafion.

resistance of a quasi-reversible system. The measured active catalyst area of the electrode is also drastically reduced to $9.1 \mathrm{~cm}^{2}$. This experiment makes it clear that, at least in the limit of very high ionic resistance in the electrode, cyclic voltammetry is able to see the deleterious influence of ionic resistance on electrode performance.

\subsection{Impedance Spectroscopy}

Electrochemical impedance spectroscopy is a technique adapted from electrical engineering to study the behavior of electrochemical systems [36]. The basic idea of the technique is to apply a small AC excitation signal to an electrochemical system and measure the $\mathrm{AC}$ response. The AC excitation signal is a small amplitude signal, so most of the behavior in the cell can be modeled using the linear approximations to the real behavior. By sweeping the frequency of the excitation signal over a wide range of frequencies, information on relative 


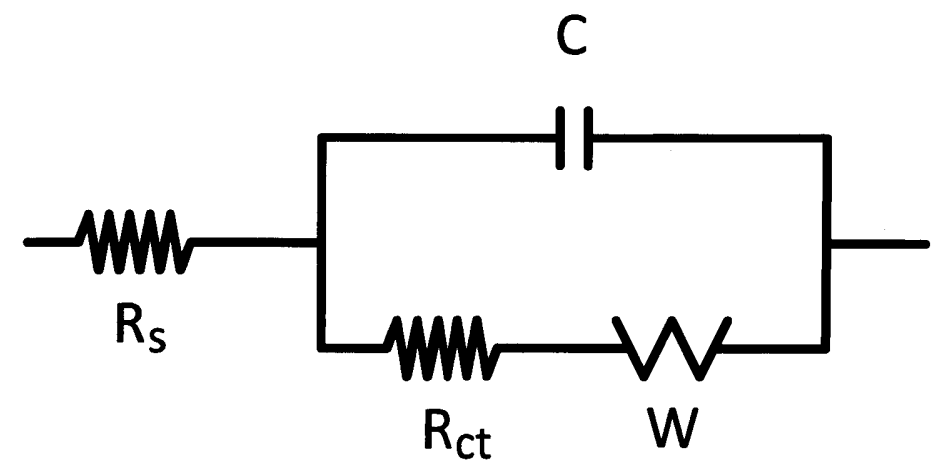

Figure 4-3: Randles equivalent circuit.

rates of the various processes in the cell can be determined.

Using the linear approximations of cell behavior, an electrochemical cell can be broken down into the linear circuit model composed of various components representing different parts of the electrochemical system. The measured spectrum can then be fit to the predicted spectrum of the equivalent circuit, allowing the determination of physical parameters within the system.

For the impedance experiments performed in this thesis, a Solartron 1260A frequency response analyzer setup to operate with two electrodes was used. A typical scan measured impedance at frequencies from $10 \mathrm{~Hz}$ to $1 \mathrm{MHz}$ with 15 points per decade in the range with an $\mathrm{AC}$ amplitude of $50 \mathrm{mV}$. All scans were taken at ambient temperature, approximately $27^{\circ} \mathrm{C}$.

A good example of the power of impedance spectroscopy is to analyze common electrochemical system model: Randles equivalent circuit, shown in Figure 4-3. This circuit consists of a series resistance, $R_{s}$, a double-layer capacitance, $C$, a charge-transfer resistance, $R_{c t}$, and a Warburg impedance, $W$. The series resistance is a model of resistance in the electrolyte, the double-layer capacitance is a model for double-layer charging, the charge-transfer resistance is a linearized model for a faradaic reaction, and the Warburg impedance is a model of mass transport impedance in the cell.

The impedance of a capacitor is [37]:

$$
Z_{C}=\frac{1}{C \omega j}
$$

while the impedance of the Warburg element is: 


$$
Z_{W}=\frac{\sigma}{\sqrt{\omega j}}
$$

where $\sigma$ is defined in Bard and Faulkner [8] as:

$$
\sigma=\frac{1}{n F A \sqrt{2}}\left(\frac{\beta_{O}}{D_{O}^{1 / 2}}-\frac{\beta_{R}}{D_{R}^{1 / 2}}\right)
$$

As seen in Equation 4.3, $\sigma$ gets smaller as the rate of diffusion increases. In the complex plane, $Z_{C}$ has constant phase angle of $-90^{\circ}$ while $Z_{W}$ has a constant phase angle of $-45^{\circ}$. Also note that the magnitudes of both $Z_{C}$ and $Z_{W}$ decrease as excitation frequency increases. It is this frequency dependence that makes it possible to parse more parameters from a single impedance spectrum than from single steady-state measurements.

Combining Equations 4.1 and 4.2 with the two resistance terms and using basic circuit equations, the total impedance of the Randles equivalent circuit is:

$$
Z=R_{s}+\frac{Z_{C}\left(R_{c t}+Z_{W}\right)}{R_{c t}+Z_{W}+Z_{C}}
$$

At very high frequencies, the impedance of the double-layer capacitance is essentially zero, and it short circuits the charge transfer and Warburg impedances, leaving the total impedance equal to just the series resistance. Low frequency behavior depends on the relative sizes of $C$ and $\sigma$. Generally, as frequency is decreased, $Z_{C}$ becomes large before $Z_{W}$ and this leads to a semi-circular region in the Nyquist plot similar to a parallel $\mathrm{RC}$ circuit. The diameter of this semicircle is equal to the charge transfer resistance, $R_{c t}$. If the Warburg impedance remains, small the overall impedance approaches $R_{s}+R_{c t}$. If the frequency is decreased low enough, the Warburg impedance will eventually dominate and the total impedance becomes $R_{s}+R_{c t}+Z_{W}$, which has a characteristic $-45^{\circ}$ slope when shown in a Nyquist plot. The transition point is determined by the relative magnitudes of the capacitive impedance, $Z_{C}$, and the Warburg impedance, $Z_{W}$. A Nyquist plot of the impedance spectra of a Randles equivalent circuit is shown in Figure 4-4 and exhibits the $-45^{\circ}$ Warburg asymptote at low frequencies, along with the high frequency purely real resistance equal to the series resistance, $R_{s}$. 


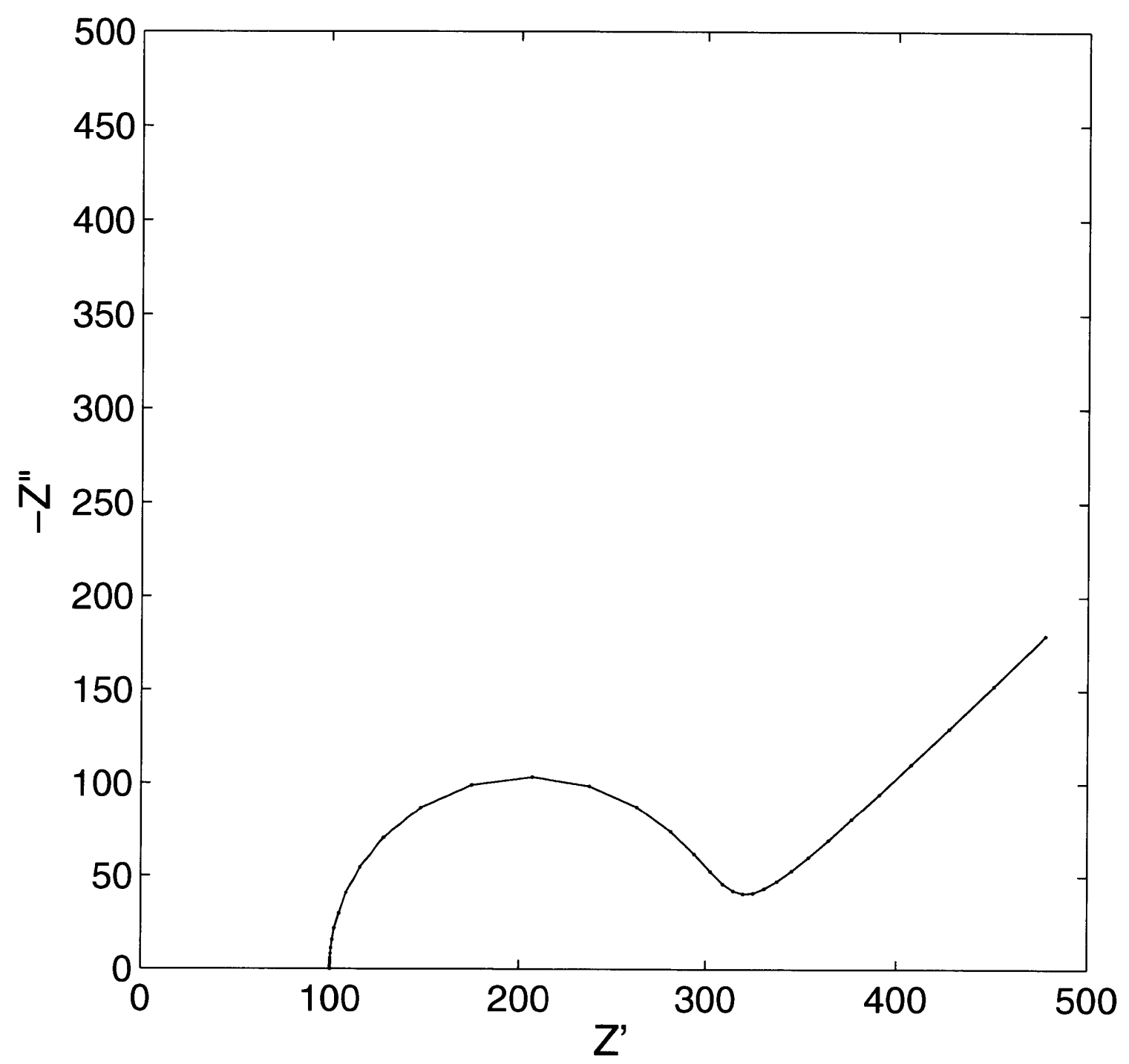

Figure 4-4: Nyquist plot of a Randles Equivalent Circuit with $R_{s}=100 \Omega, R_{c t}=200 \Omega$, $C=10 \mu \mathrm{F}$, and $\sigma=200 \Omega / \sqrt{\mathrm{s}}$ 


\subsubsection{Capacitance Plots of Impedance Data}

There are many options for the graphic display of impedance spectroscopy data including the extremely popular Nyquist and Bode plots. A relatively lesser known option is called the capacitance plot. A capacitance plot is similar to a Nyquist plot, but the imaginary impedance component on the vertical axis is replaced with an effective capacitance value defined by:

$$
C_{e f f}=\frac{-1}{Z " \cdot \omega}
$$

where $Z$ " is the imaginary component of cell impedance and $\omega$ is the excitation frequency. Capacitance plots have particular utility when dealing with blocking electrodes that exhibit capacitive or capacitor-like behavior in the low-frequency limit. The solid-electrolyte cells described in this thesis are exactly this type of cell.

There are two features of particular interest when examining a capacitance plot: the initial slope of the line and the final asymptote. The initial slope gives an approximation of the conductivity within the electrode. The steeper the initial slope, the higher the ionic conductivity. The asymptotic value for capacitance at low frequencies is driven by the area and activity of catalyst that is electrochemically accessible in the electrode. The higher the asymptotic capacitance value, the larger the amount of catalyst area utilized by the electrode. Capacitance plots have been used by Pickup et al [38, 39] to study porous electrodes similar to those used for impedance experiments in this thesis.

\subsubsection{Transmission Line Data Fitting}

In Chapter 3, a transmission-line model with constant-phase elements in the charge transfer branch is proposed as a model for impedance spectroscopy in porous electrodes. This model has a closed form solution for impedance that makes it a good option for data fitting. All the data fits of impedance data in this thesis used this model. The data were fit using constrained minimization in Matlab (fmincon.m) to minimize the objective function

$$
\mathrm{F}(\alpha)=\sqrt{\sum\left|\mathrm{Z}_{T L}\left(\alpha, \omega_{i}\right)-Z_{i}\right|^{2}}
$$

where $\alpha$ is an array of model parameters, $Z_{i}$ is the impedance data point a frequency $\omega_{i}$, 


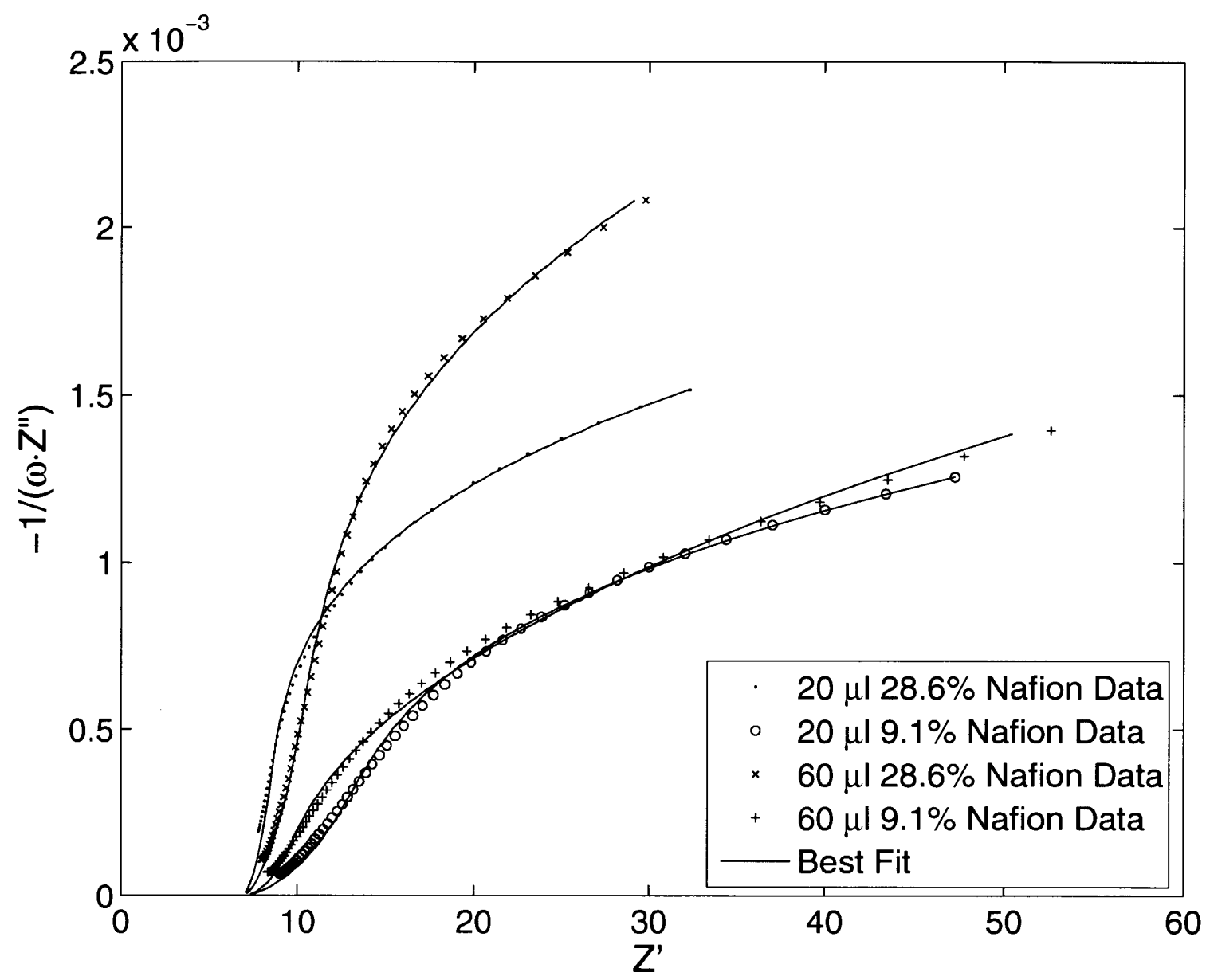

Figure 4-5: Capacitance plots of standard electrode data and best fit with a modified transmission line.

and $\mathrm{Z}_{T L}\left(\alpha, \omega_{i}\right)$ is the calculated transmission line impedance for the specified frequency and model parameters. There are four parameters that compose the vector $\alpha$ : the series resistance, $R_{s}$; the total ionic resistance, $R$; the CPE capacitance-like term, $T$; and the phase exponent, $\phi$, of the CPE element, which takes values between 0 and 1 .

Figure 4-5 shows the capacitance plots of the impedance data from several cells plotted with the lines of best fit using the transmission line model. The quality of this fit suggests that the transmission line is a reasonable model for the impedance behavior of fuel cell electrodes. The best fit parameters along with the normalized error, $F_{\min }$, for each of the four electrodes, shown in Figure 4-5, are shown in Table 4.1. The parameters of particular interest for evaluating electrode performance are the ionic resistance, $R$, and the capacitancelike term, $T$. The ionic resistance term represents the total ionic resistance in the electrode; it is expected to grow with electrode thickness. The capacitance-like term scales with the 
Table 4.1: Best fit parameters the modified transmission line model on standard electrodes.

\begin{tabular}{|c|c|c|c|c|c|}
\hline & $R_{s}(\Omega)$ & $R(\Omega)$ & $T$ & $\phi$ & $F_{\min }(\Omega)$ \\
\hline \hline $20 \mu \mathrm{L}$ Standard Ink & 6.810 & 4.320 & 0.0024 & 0.778 & 1.178 \\
\hline $20 \mu$ LLow-Nafion Ink & 6.300 & 6.798 & 0.0021 & 0.738 & 2.827 \\
\hline $60 \mu \mathrm{L}$ Standard Ink & 7.683 & 9.608 & 0.0034 & 0.760 & 1.587 \\
\hline $60 \mu \mathrm{L}$ Low-Nafion Ink & 6.945 & 16.825 & 0.0026 & 0.669 & 4.110 \\
\hline
\end{tabular}

available catalyst area within the electrode.

\subsection{Cell Setup}

Although a full fuel cell can be fabricated incorporating the ion-conducting channels in the anode, none of the experimental validation of the electrode was performed with a full fuel cell. A full cell has many components and operating conditions that can impact overall performance. Drawing meaningful conclusions based on full cell performance requires either very repeatable cell manufacturing, or performing many replicates with each cell to eliminate wide fabrication variations. Both options proved impractical, so designing ex-situ experiments to measure the anode performance directly is preferred. The experiments presented in this thesis were performed using the cells described in this section.

\subsubsection{Three-Electrode Cell with Aqueous Electrolyte}

The three-electrode cell is one of the most popular methods to isolate an electrochemical behavior of interest for study. The cell consists of a working electrode, at which the reaction of interest takes place; a reference electrode, which can maintain a repeatable and constant potential, but draws a minimum current; and counter electrode, which is used to supply whatever current is required by the working electrode. The use of a separate reference electrode allows much of the solution resistance to be eliminated from a cell measurement and it allows the counter electrode to be any electrode that does not introduce contaminants into the cell.

In the experiments presented in this thesis, the three electrodes were submerged in an aqueous electrolyte and maintained under an inert atmosphere of nitrogen. The threeelectrode cell was prepared in a 5-port reaction flask (Ace Glass 9473-15). To setup the cell, first a liquid electrolyte was poured into the reaction flask; typically $80 \mathrm{~mL}$ of electrolyte was 


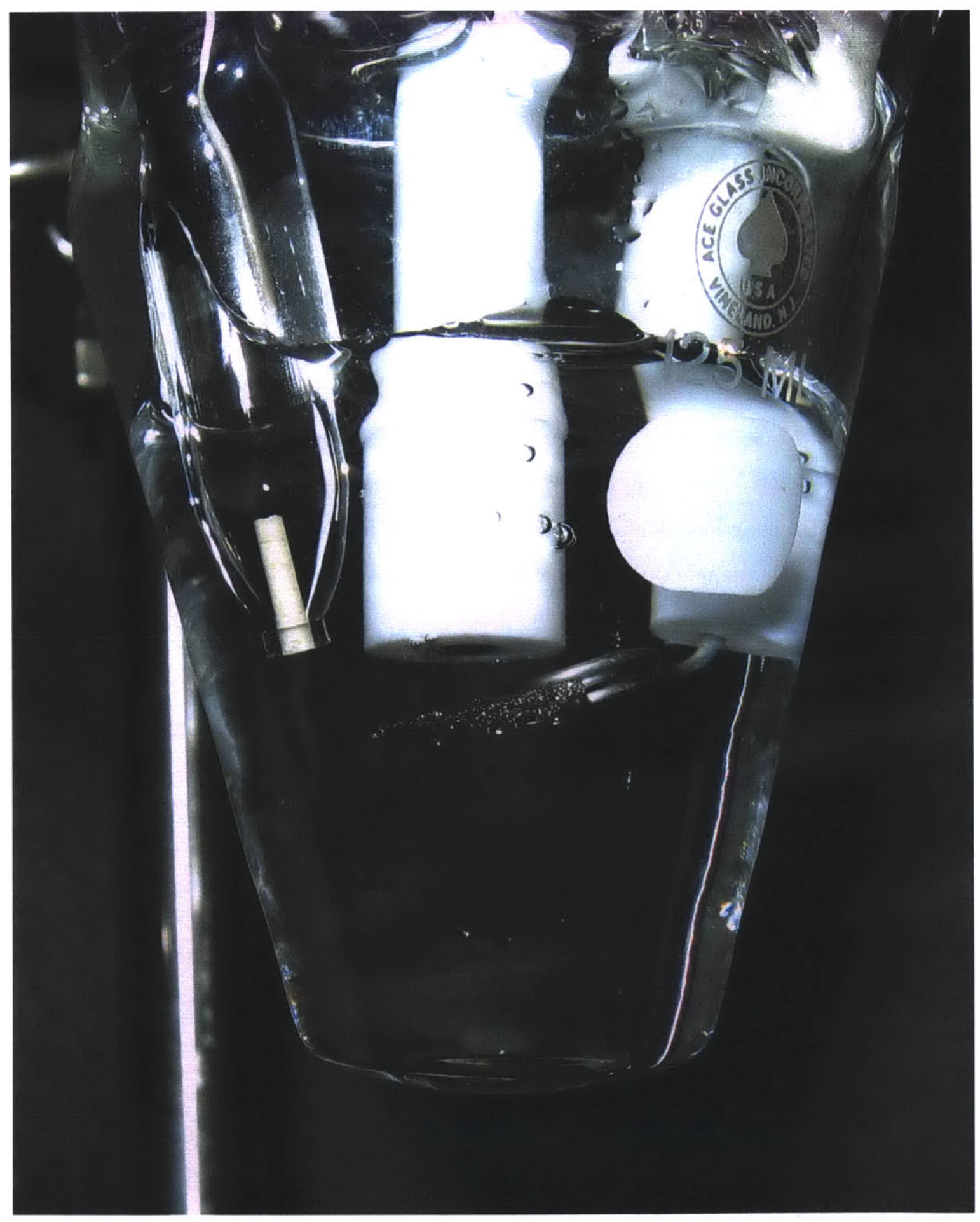

Figure 4-6: Photograph of the three-electrode cell with aqueous electrolyte. 
used in each experiment. The electrolyte used in most of this thesis was $0.5 \mathrm{M}$ sulfuric acid in water but some experiments were performed using a mixture of $0.5 \mathrm{M}$ sulfuric acid and $1 \mathrm{M}$ methanol in water. The electrode to be tested was then inserted into the center port of the flask such that the tip of the electrode did not reach the top of the solution. A platinum mesh counter electrode and a $\mathrm{Ag} / \mathrm{AgCl}$ reference electrode were then inserted into the side ports of the flask. A plug with a small vent hole was inserted into one of the threaded side ports. A fritted glass diffusion rod was inserted into the other threaded flask ports and inert gas, usually nitrogen, was bubbled through the electrolyte to eliminate any dissolved oxygen. The purge process lasted for approximately 30 minutes and then the diffusion rod was pulled out of the electrolyte solution, but left in the flask to maintain a nitrogen environment in the flask. The working electrode was then inserted into the electrolyte solution. The reference and the working electrode's insertion depths were adjusted to bring the reference electrode as close to the working electrode as possible, though this adjustment was far from repeatable. This process left the cell ready to start the cyclic voltammetry process described in Section 4.1. An assembled three-electrode cell is shown in Figure 4-6, with the reference electrode on the left, the working electrode in white in the center, and the counter electrode directly below the working electrode.

Though mostly used for cyclic voltammetry experiments in this thesis, the three-electrode cell was also briefly used for impedance spectroscopy as well as a transition to the solid electrolyte cells described below. Details on the impedance spectroscopy results using the three-electrode cell can be found in Section B.2 of the Appendix.

\subsubsection{Solid Electrolyte Impedance Cells}

All of the impedance spectroscopy experiments in this thesis were performed with solid electrolyte impedance cells. These cells consisted of a Nafion membrane to serve as the electrolyte with a small working electrode applied to one side and a much larger counter electrode on the other side.

These cells were tested using two different fixtures: one with a fixed current collector at the working electrode and a second fixture that uses a movable plunger as the current collector at the working electrode. The fixture with the movable plunger proved far superior and all the results presented in this thesis used this fixture exclusively. The details of the apparatus with the rigid current collector, along with the associated impedance spectroscopy 


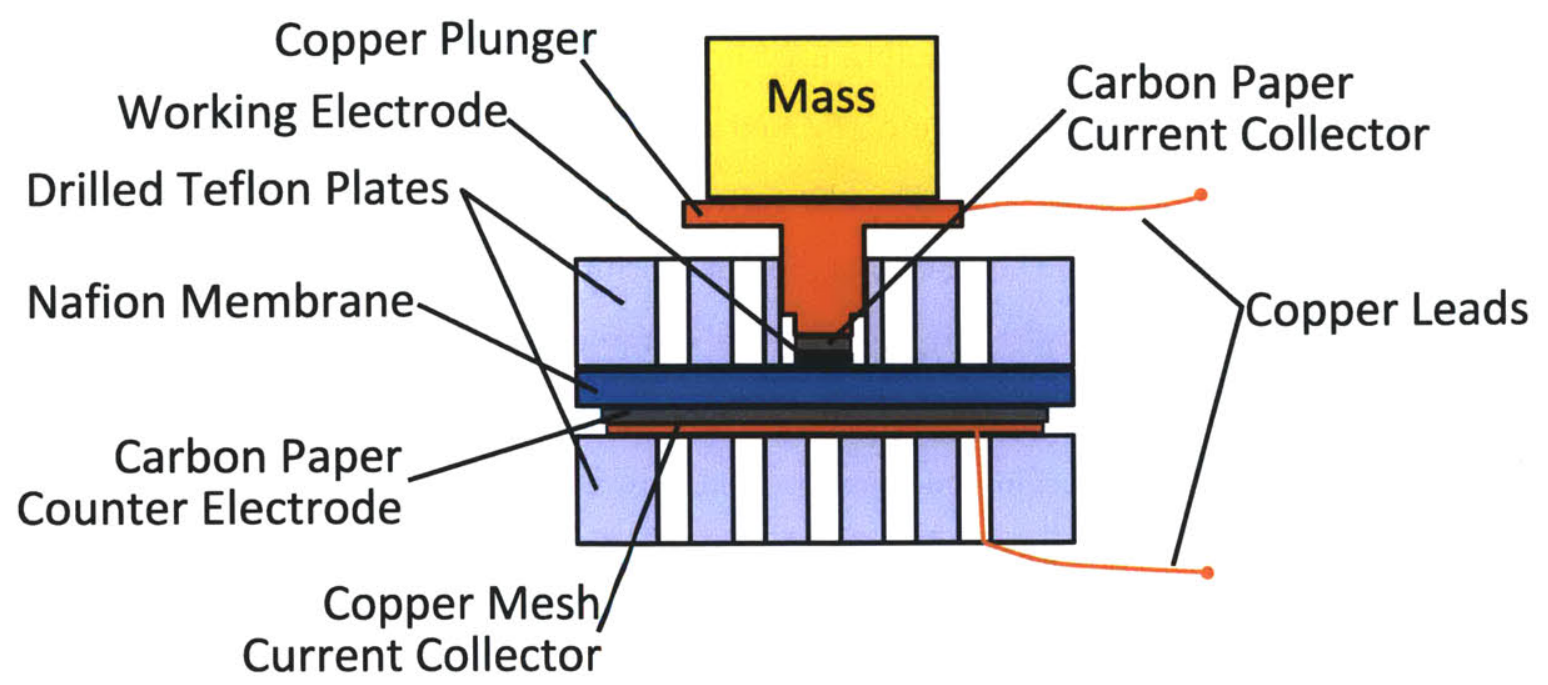

Figure 4-7: Second generation of solid electrolyte impedance cell.

results that lead to the final cell and fixture design, can be found in Appendix A.

In this impedance cell, air had access to both the working and counter electrodes. Despite the lack of a completely inert environment, there was no faradaic reaction at the electrodes in the impedance experiment. As a result, the charge transfer behavior of these cells can best be modeled as that of a blocking electrode. This becomes clear from the experimental results presented in Chapter 5, in which Nyquist plots do not show a low-frequency return to the real axis, as would be expected with a faradaic reaction present. The identical feed gas at each electrode also meant that all the impedance measurements had to be taken without any DC bias voltage. The use of a solid electrolyte required that the cells be tested in a humidified environment so that the electrolyte would conduct hydrogen ions. As such, all solid electrolyte experiments were performed in a humidity controlled chamber.

The fixture used to hold solid electrolyte cells, shown in Figure 4-7, used a movable current collector on the working electrode side with a rigid current collector on the counter electrode side. The main feature of this fixture is the copper plunger used as a current collector. The plunger is a piece of copper turned on a lathe such that it has the shape of a "T." The top of the plunger has a $25.4 \mathrm{~mm}$ diameter while the shaft has a $6.4 \mathrm{~mm}(1 / 4 \mathrm{in})$ diameter. The tip of the plunger is turned to a diameter of $4 \mathrm{~mm}$ and a disc of carbon paper is affixed to the tip using conductive silver epoxy. The carbon paper tip prevents the copper plunger from directly contacting the electrode material.

For impedance experiments, the cell was contained between drilled Teflon plates, which 
are $9.5 \mathrm{~mm}(3 / 8 \mathrm{in})$ thick. The thicker plate was needed on the working electrode side, as it also serves to guide the copper plunger. A $6.4 \mathrm{~mm}(1 / 4 \mathrm{in})$ diameter hole was drilled in the center of one of the Teflon plates to match the $6.4 \mathrm{~mm}(1 / 4 \mathrm{in})$ diameter shaft of the copper plunger. This allowed the plunger to slide freely in the fixture without toppling over. While in use, a small weight was placed atop the copper plunger to exert a constant force on the working electrode. The two sides of the fixture were held together with rubber bands exerting a very light force sufficient only to keep the cell in place. The electronic connection to the counter electrode was established with a copper mesh placed behind the carbon paper counter electrode and a wire lead was soldered to the mesh. This allowed for evenly distributed contact points across the carbon paper counter electrode and no potential for copper directly contacting the membrane.

\subsection{Electrode Fabrication}

\subsubsection{Catalyst Ink}

All electrodes used in this thesis were formed from dispersed catalyst inks that were then dried. The ink was a mixture of catalyst powder, Nafion solution, and deionized water. The catalyst powders used in this thesis were from E-Tek and consist of carbon black (XC-72) with catalyst particles deposited on the carbon black. The catalyst powders were loaded with between 40 and 60 percent catalyst by weight and the balance was carbon black. The ink was mixed by first measuring out a small amount, typically $25 \mathrm{mg}$, of catalyst powder using a Mettler AT20 precision scale. The water was then added to the ink. Typical inks used $6 \mathrm{~mL}$ of deionized water, though some inks used to achieve higher loading used less water. The water and powder were mixed for approximately 10 minutes with a magnetic stirrer (VWR \#12365-382). Nafion solution (5\%) was added to the ink last, using a VWR VE-20 micropipette, while the ink was being mixed with a magnetic stirrer. The volume of Nafion added was varied for several inks, but the most common and most effective ink used $0.2 \mathrm{~mL}$ of $5 \%$ Nafion solution. After the Nafion was added, the ink was sonicated in a Branson 1210 ultrasonic cleaner for 1 hour to complete the mixing procedure. The contents of the two most common inks used in this thesis are shown in Table 4.2. 
Table 4.2: Components of common catalyst ink mixtures used in this thesis.

\begin{tabular}{|c|c|c|c|c|}
\hline & Nafion Content & Catalyst Powder Mass & Water & $5 \%$ Nafion Solution \\
\hline \hline Standard Ink & $28.6 \%$ wt. & $25 \mathrm{mg}$ & $6 \mathrm{~mL}$ & $0.2 \mathrm{~mL}$ \\
\hline Low-Nafion Ink & $9.1 \%$ wt. & $25 \mathrm{mg}$ & $6 \mathrm{~mL}$ & $0.05 \mathrm{~mL}$ \\
\hline
\end{tabular}

\subsubsection{Glassy Carbon Electrodes}

Electrodes to be studied using cyclic voltammetry were built on glassy carbon electrode supports. The supports consisted of a $4 \mathrm{~mm}$ diameter glassy carbon rod that was press fit into a Teflon sheath. Electrical contact between the control hardware and the working electrode was established through a threaded stainless steel shaft. The shaft was threaded into the back of the Teflon sheath that holds the glassy carbon electrode support. The shaft compressed a stainless steel spring into the exposed back of the glassy carbon to make electrical contact with the support.

\subsubsection{Standard Electrodes}

The simplest fabrication process was that employed for the standard electrodes on glassy carbon supports. The electrodes were formed from the electrode ink described in Section 4.4.1. The ink was then magnetically stirred for 20 minutes and then sonicated for approximately an hour before use. A droplet of the resulting ink was applied to the working electrode support using a micropipette. The electrode was allowed to dry over night so that only the Nafion and catalyst powder remain. Catalyst loading was determined by the volume of ink applied.

\subsubsection{Structured Electrodes}

The fabrication process for the Nafion post electrodes on glassy carbon supports was quite a bit more involved than for the standard electrodes. The process began with the machining of an aluminum mold for the posts. The mold was cut with a Microlution 363-S micromill from $177.8 \mu \mathrm{m}(0.007 \mathrm{in})$ thick aluminum sheet. The aluminum was affixed to the flat fixture with Intertape-592 double-sided tape and compressed in place by an aluminum plate tightened over the sheet with four bolts. After holding the sheet in place for a few moments the plate was removed and the sheet was ready for milling. The compliance of the tape resulted in poor precision when the milling machine touched off on the part to measure the top of the 
stock and set the zero reference. This meant that the final mold thickness could vary and that there was a risk of puncturing the aluminum mold sheet if the zero plane were set too deep into the material. The aluminum sheet was faced with a $3.2 \mathrm{~mm}(1 / 8 \mathrm{in})$ endmill then holes were drilled in the aluminum using a small endmill. Tools are available with diameters as low as $25.4 \mu \mathrm{m}(0.001 \mathrm{in})$, but most of the electrodes in this thesis used $76.2 \mu \mathrm{m}(0.003 \mathrm{in})$ tools. Typical hole depth was set to $100 \mu \mathrm{m}$. Each mold took approximately 35 minutes of machining to cut.

Once the tool was cut, it was cleaned with isopropyl alcohol and an ultrasonic cleaner. The molds were then dried with compressed air. A drop or two of $10 \%$ Nafion solution was applied to the mold and gently spread to cover the entire array of holes. The mold was placed in a Bel-Art 42012000 vacuum chamber and a vacuum was drawn for about 30 seconds, then released several times to ensure that the holes were completely filled with Nafion solution. The mold was then placed in an oven at $50^{\circ} \mathrm{C}$ for an hour until the solvent in the Nafion solution had evaporated. A drop of isopropyl alcohol was then applied to the top of the Nafion to soften it so it could bind with the carbon paper backing. A piece of SpectraCarb 2050-L carbon paper was then placed on top of the mold and another drop of isopropyl alcohol was added to the top of the carbon paper. The mold and carbon paper were then sandwiched between $3.2 \mathrm{~mm}(1 / 8$ in) thick Teflon sheets and compressed in a bench vise overnight.

The mold and the carbon paper were removed from the Teflon gently using a razor blade so that the carbon paper did not stick to the Teflon sheet. Once the mold and carbon paper sandwich was removed from the Teflon, it was soaked in $1.5 \mathrm{M}$ sodium hydroxide for approximately 15-20 minutes to dissolve the aluminum mold. The carbon paper was removed from the solution when it separated from the aluminum, and was rinsed thoroughly in deionized water. The result was an array of well-formed Nafion posts on a sheet of carbon paper shown in Figures 4-8 and 4-9. In Figure 4-8, the focal plane of the microscope is at the top of the Nafion posts. The posts are well-formed $75 \mu \mathrm{m}$ diameter posts in a rectangular grid pattern with $175 \mu \mathrm{m}$ spacing. On the top of the posts, the circular machining lines from the bottom of the mold holes are clearly replicated, indicating that the Nafion filled the full depth of the mold and did not break during mold removal. In Figure 4-9, the image is focused at the base of the very same set of posts. Again, machining lines are visible in between the carbon paper fibers, indicating the Nafion has filled the pores of the carbon 
paper. The fibers of the carbon paper can also be seen protruding through the Nafion, suggesting that electronic connection with the electrode ink should be possible.

With the posts formed, the electrode was then attached to the glassy carbon working electrode support. To do so a disc of Nafion posts was cut from the array of posts using a $4 \mathrm{~mm}$ diameter punch. The disc was attached to the glassy carbon using a non-catalyzed ink as glue. The ink contained only Nafion and carbon black (XC-72). A $4 \mu \mathrm{L}$ drop of the glue ink was applied to the glassy carbon support and the carbon paper disc with Nafion posts was gently pressed onto the ink. The electrode was then allowed to dry overnight. Once the Nafion posts were attached to the glassy carbon, the same catalyst ink used for the standard electrodes was applied to the Nafion posts. Droplets tended not to wet the full depth of the posts and needed to be physically spread with the tip of the micropipette to attempt to cover the full area of posts. Despite these efforts, the catalyst coverage for the Nafion post electrodes was much worse than in the standard electrodes. Additionally, the bond between the carbon paper and the glassy carbon was quite weak, making the electrodes susceptible to breaking with any impact.

\subsubsection{Through-Plane Electrodes}

Through-plane impedance cells were fabricated from a commercially purchased Nafion 117 membrane. The membrane was cut into $38 \mathrm{~mm}$ (1.5 in) square pieces. The counter electrode was then applied to one side of the membrane and the working electrode was applied to the other, with counter electrode having a much larger area than the working electrode, so that its measured impedance remained low in comparison to the working electrode. The counter electrode was either an ink-based counter electrode or a carbon paper-based counter electrode. Carbon paper-based counter electrodes produced significantly better results than the ink-based counter electrodes so only carbon paper-based counter electrodes are described here. The fabrication process for ink-based counter electrodes is described in Section A.1.2 of the Appendix. The working electrodes were all ink-based; however, some of the working electrodes used were standard electrodes with no internal structure, and some of them were structured electrodes with Nafion channels running through the electrode. The fabrication procedure for the cells changed based on the particular combination of counter electrode: ink-based or carbon paper-based, and working electrode: standard or structured, used in the cell. 


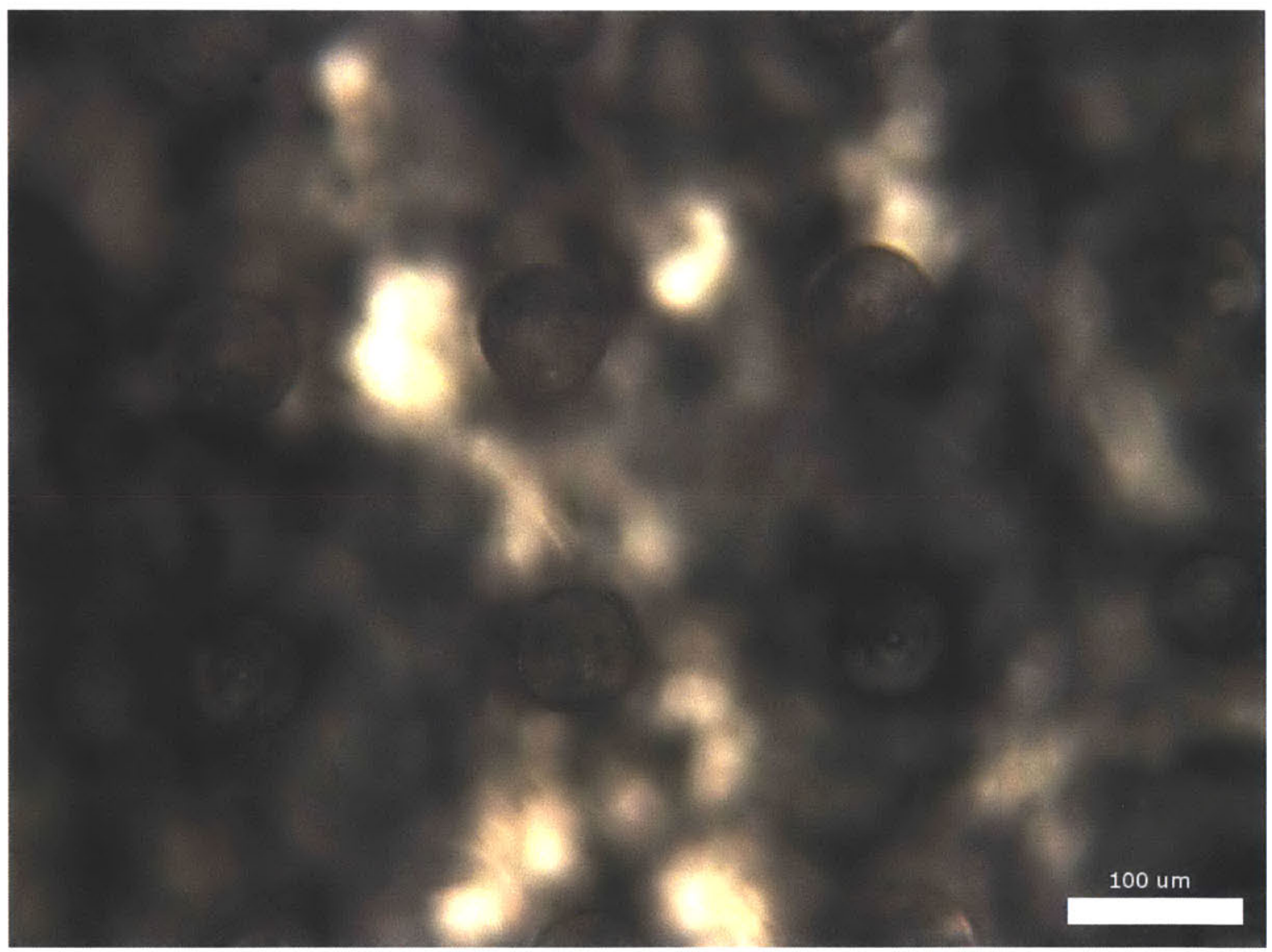

Figure 4-8: Cast Nafion posts on carbon paper with the focal plane at the top of the posts. 


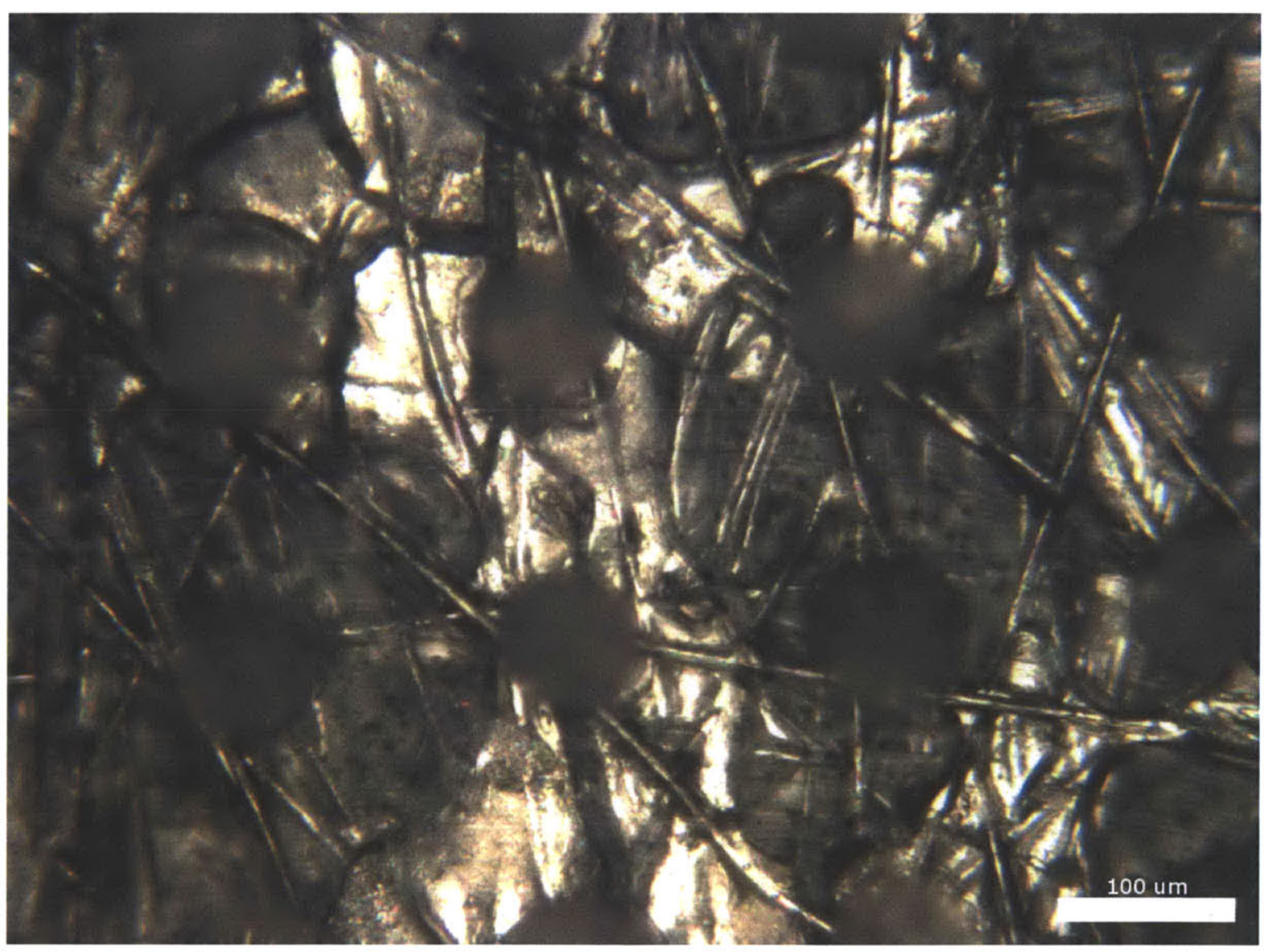

Figure 4-9: Cast Nafion posts on carbon paper with the focal plane at the bottom of the posts. 


\subsubsection{Carbon Paper-Based Counter Electrodes}

The main type of counter electrode used in this study was the carbon paper-based counter electrode. Preparation of this type of electrode began by cutting the SpectraCarb 2050-L carbon paper into $38 \mathrm{~mm}$ (1.5 in) squares. Catalyst ink was then applied to the carbon paper to provide sufficient activity to reduce impedance to insignificant levels. The ink used was identical to the standard Nafion loading ink used for the best performing electrode in this thesis. When dry, the ink contains $28.6 \%$ Nafion by mass, with the balance being $60 \%$ by mass platinum catalyst powder supported on XC-72 carbon black. Approximately 1 gram of catalyst ink was applied to each square of carbon paper and spread over the full area with a paintbrush. The ink was allowed to dry overnight before the catalyzed carbon papers are pressed onto membranes.

The hot-pressing of the counter electrodes onto the membrane began with soaking the Nafion membrane in deionized water for 10-20 minutes. The membrane was removed from the water and excess drops were shaken from the membrane. The membrane was laid on a piece of aluminum foil covering a flat stainless steel 'mold.' The counter electrode was formed from carbon paper cut into a $38 \mathrm{~mm}$ (1.5in) square to match the Nafion membrane. To improve the connection between the carbon paper and the membrane, several drops of $10 \%$ Nafion solution were applied to the carbon paper and spread using a paintbrush. The carbon paper was then placed, Nafion-side down, on the Nafion membrane and a flat stainless steel mold was placed above the carbon paper. The mold assembly was then placed in a Carver 4386 heated press. The assembly was pressed for 4 minutes with a force of $8.9 \mathrm{kN}$ at a temperature of $130^{\circ} \mathrm{C}$. After the pressing operation was completed, the cell was removed from the mold assembly. The stainless steel easily separated from both the aluminum foil and from the carbon paper side of the cell. The aluminum foil, however, was typically still adhered to the Nafion side of the cell. To remove the aluminum foil, the cell was placed carbon paper-side down on the stainless steel mold. The aluminum was then peeled back from the Nafion while the cell was held flat against the stainless plate. This arrangement allowed the aluminum to peel away from the Nafion without cracking or damaging the carbon paper counter electrode.

A few carbon paper counter electrodes were made without catalyst ink, but resulted in larger than acceptable impedance measurements, described in Section A.2.4 of the Appendix, 
and were no longer used. These electrodes were made with the identical process described above, but without the catalyst ink.

\subsubsection{Standard Working Electrodes}

After the counter electrode was applied to the cell, the working electrode was applied. The cell was placed, counter electrode-side down on a stainless steel backing plate and a $3.2 \mathrm{~mm}$ $(1 / 8$ in) thick Teflon mask was placed above the cell to set the working electrode area. The Teflon mask was a circle milled in the center of a Teflon plate using a $15^{\circ}$ tapered endmill. The diameter at the base of the mask was $4 \mathrm{~mm}$. The mask was clamped in place with four binder clips and the whole assembly was placed in a desiccator. Catalyst ink was applied to the membrane using a micropipette to specify the precise loading for each cell. The electrode was dried overnight in the desiccator and the Teflon mask and stainless steel backing plate were removed to yield a full cell, ready for testing.

Complications with electrode peeling arose when making working electrodes that had high Nafion content in the ink and/or high catalyst loading in the total working electrode. The edges of the electrode peeled away from the membrane when the electrode contained more than $28.6 \%$ Nafion or when more than $60 \mu \mathrm{L}$ of ink was applied to the electrode. This was most likely caused by the larger amount of Nafion in the electrode contracting, as it dried out, and pulling the electrode together. This could be largely avoided by applying large volumes of ink in two batches, rather than all at once.

\subsubsection{Structured Working Electrodes}

The shift to catalyzed carbon paper counter electrode from ink-based counter electrodes required some modification to the fabrication process for structured electrode cells described in Section A.1.2.1 of the Appendix. The structured cell required two hot pressing operations, rather than just the one in a standard cell, to attach the counter electrode. The carbon paper could not survive the higher force required to create the Nafion posts, so the posts had to be embossed first, before the carbon paper counter electrode was pressed in place. In order to avoid damaging the embossed posts, the membrane was not removed from the post mold until after the carbon paper had been attached. The removal process was difficult, because the carbon paper turned the normally pliable membrane into a fairly rigid sheet that was far too brittle to survive attempts to demold the post. When the membrane was embossed 


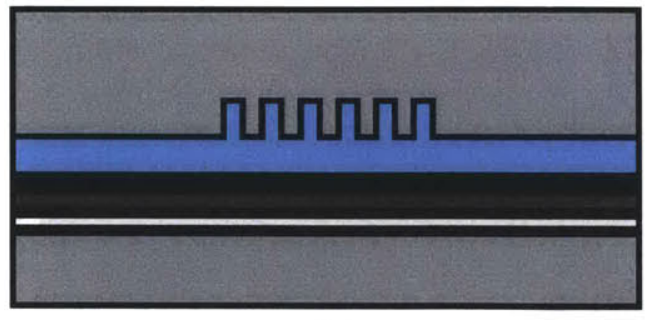

(a)

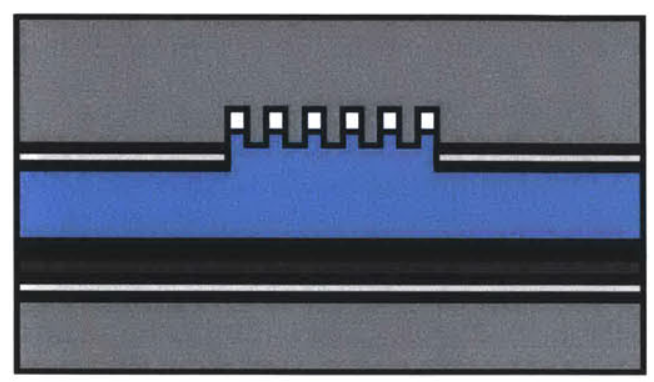

(c)

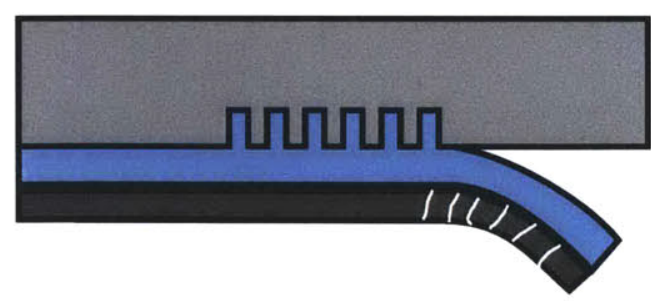

(b)

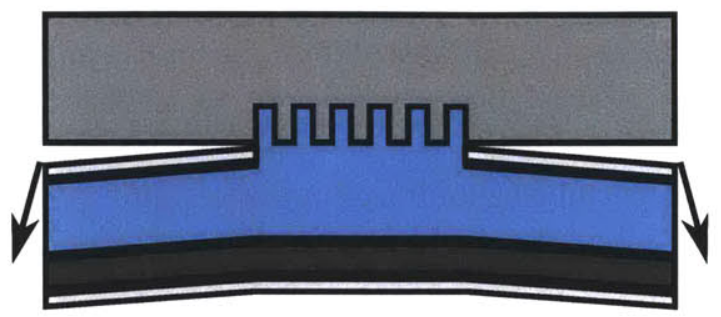

(d)

Figure 4-10: Diagrams of fabrication challenges for structured cells with carbon paper counter electrodes.

directly against the micromilled mold, as in Figure 4-10a, attempts to peel the membrane from the mold resulted in the counter electrode shattering, as in Figure 4-10b. The addition of a layer of aluminum foil with a cutout for the textured region between the membrane and the post mold allowed the membrane to be removed from the mold without shattering the structure, as shown in Figure 4-10d. This method, however, resulted in poorly formed posts because the additional thickness of the aluminum foil was enough to prevent complete mold filling, as shown in Figure 4-10c.

The demolding issues were alleviated by slightly redesigning the mold so that the holes used to form the posts were on a circular raised region $11.1 \mathrm{~mm}(7 / 16 \mathrm{in})$ in diameter. Prior to molding, a piece of aluminum foil with $11.1 \mathrm{~mm}$ (7/16 in) hole punched in it was placed over the mold to allow for easy separation of the Nafion edges from the mold. A diagram of this arrangement is shown in Figure 4-11. With the majority of the Nafion membrane easy to remove from the mold and only the textured region still attached, demolding could be achieved without excessive bending of the carbon paper, thus ensuring that it does not crack.

The mold to emboss the electrode texture onto the membrane was cut from a $38 \mathrm{~mm}(1.5 \mathrm{in})$ square piece of $1.6 \mathrm{~mm}(1 / 16 \mathrm{in})$ thick aluminum using a Microlution 363-S micromilling ma- 


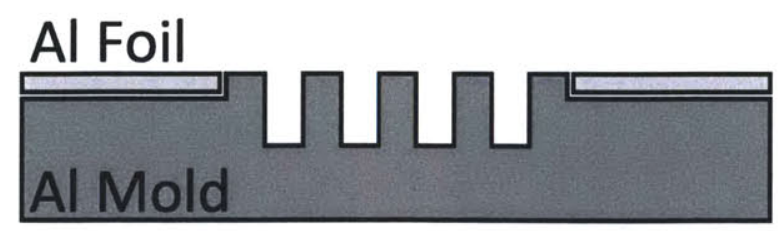

Figure 4-11: Diagram of the stepped mold and foil combination used to emboss structured electrodes that use carbon paper counter electrodes.

chine. The aluminum plate was attached to the micromill pallet using Intertape-592 doublesided tape. Unlike cutting the mold into $177.8 \mu \mathrm{m}(0.007 \mathrm{in})$ thick aluminum sheet, there was no threat of punching through the $1.6 \mathrm{~mm}(1 / 16 \mathrm{in})$ aluminum plate with the drill bit. As a result, the aluminum plate could be faced more aggressively ensuring that the full plate was planar without worrying about making it too thin in any region. The aluminum was drilled with $76.2 \mu \mathrm{m}$ (0.003 in) diameter holes $100 \mu \mathrm{m}$ deep with a center-to-center spacing of either $175 \mu \mathrm{m}$ or $300 \mu \mathrm{m}$. A $11.1 \mathrm{~mm}(7 / 16 \mathrm{in})$ circle surrounding the patterned section was left untouched while the remainder of the mold was faced another $75 \mu \mathrm{m}$ deep to provide room for the aluminum foil mold release layer.

The fabrication process for structured cells with carbon paper electrodes is shown schematically in Figure 4-12. As described in Section 4.4.3.1, 1 gram of catalyst ink was applied to each $38 \mathrm{~mm}$ (1.5 in) square of carbon paper and allowed to dry, as shown in Figures 4-12a and $4-12 \mathrm{~b}$. The Nafion membrane was soaked in deionized water for 10-20 minutes and then placed on a piece of aluminum foil covering a stainless steel backing plate. A piece of aluminum foil with a $11.1 \mathrm{~mm}(1 / 16 \mathrm{in})$ hole punched in it was placed over the electrode mold such that the textured region showed through the cutout. The mold was then placed face-down on the membrane, as shown in Figure 4-12c. The mold assembly was then pressed for 6 minutes at a temperature of $130^{\circ} \mathrm{C}$ under a load of $111.2 \mathrm{kN}$, which was the maximum this press could provide. Because the mold has a larger area in this case than in the case of the ink-based counter electrodes, the load of $66.7 \mathrm{kN}$ for that process would need to be more than doubled to keep the embossing pressure constant. The capacity of the press used in this thesis made that impossible, so the maximum force was applied and the emboss time was extended slightly to compensate.

After the texture had been embossed, the mold assembly was removed from the press. The stainless steel backing plate was removed and the aluminum foil on the flat side of the membrane was peeled off. Meanwhile, several drops of $10 \%$ Nafion solution were applied 
to the catalyzed side of a piece of carbon paper and spread over the entire area with a paintbrush. The carbon paper was then placed, Nafion side down, on the Nafion membrane. A new piece of aluminum foil was placed over the back of the carbon paper and a stainless steel backing plate was placed on top of that, as shown in Figure 4-12d. The whole assembly was returned to the heated press and pressed for 4 minutes at $130^{\circ} \mathrm{C}$ under $8.9 \mathrm{kN}$ of load. The mold assembly was removed from the press and the membrane was removed from the mold by pulling on the aluminum foil to keep the membrane and carbon paper roughly straight as the mold was removed, as shown in Figure 4-12e. The aluminum foil was then peeled from both sides of membrane, as shown in Figure 4-12f.

The working electrode ink was applied just like the standard electrode. A mask with a tapered $4 \mathrm{~mm}$ diameter opening in $3.2 \mathrm{~mm}(1 / 8 \mathrm{in})$ thick Teflon sheet was clamped onto the structured membrane. The desired amount of catalyst was applied to the structure using a micropipette, as shown in Figure 4-12g. The ink was dried overnight in a desiccator. The Teflon mask was then removed to reveal a completed structured cell, as shown in Figure 4$12 \mathrm{~h}$. 


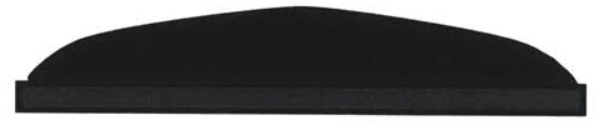

(a)

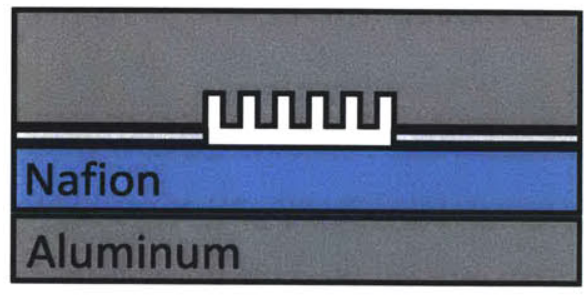

(c)

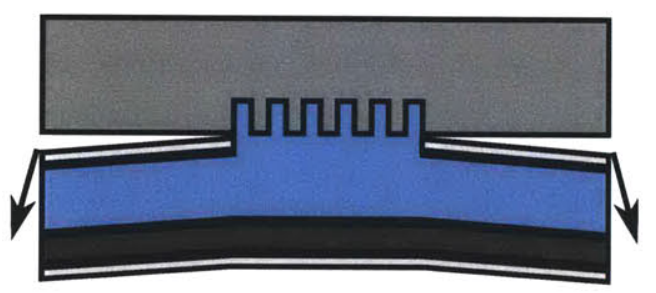

(e)

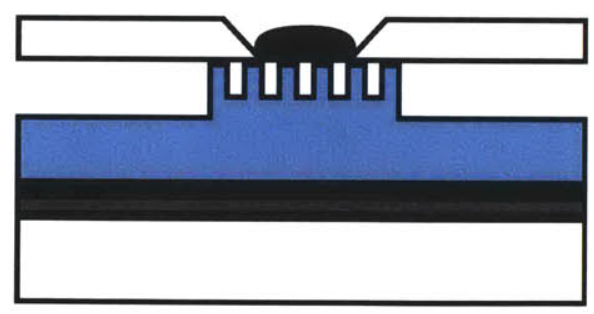

(g) (b)

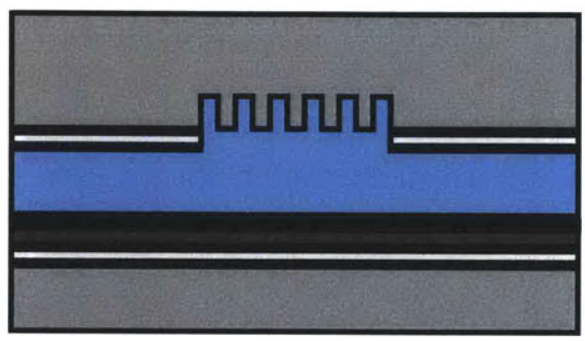

(d)

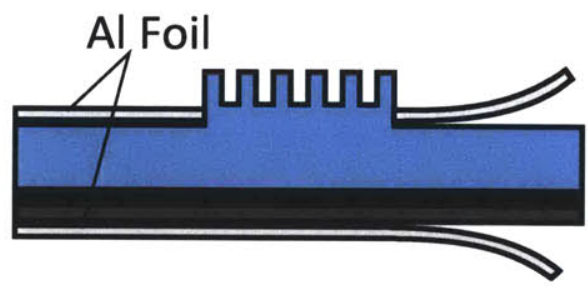

(f)

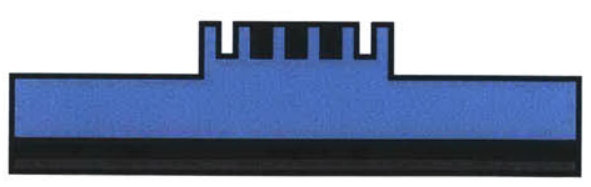

(h)

Figure 4-12: Process used to fabricate structured cells with catalyzed carbon paper counter electrodes. 


\section{Chapter 5}

\section{Results and Discussion}

\subsection{Influence of Nafion Content on Catalyst Availability}

One straightforward way to improve ionic conductivity in porous carbon electrodes is to simply add more Nafion to the ink used to form the electrode. More Nafion means more and/or thicker ionically conductive channels in the electrode and therefore conductivity should improve. Axiomatic Design analysis presented in Section 3.1.2, however, shows that additional Nafion can have deleterious effects on other electrode functions, most notably, the amount of catalyst available in the electrode. It is therefore interesting to look at the effect that Nafion loading in the electrode has on active catalyst area available.

\subsubsection{Determination of Ideal Nafion Loading with Cyclic Voltammetry}

Cyclic voltammetry provides a convenient method, described in Section 4.1.1, to measure the active catalyst surface area of a given electrode. Figure 5-1 show the cyclic voltagrams of electrodes all made with $0.3 \mathrm{mg} / \mathrm{cm}^{2}$ catalyst loading and progressively larger amounts of Nafion. It is clear from the $4.8 \%$ Nafion voltagram that too little Nafion will drastically reduce catalyst availability. This electrode shows significantly smaller hydrogen adsorption peaks that are not particularly sharp, indicating higher resistance. Increasing Nafion content leads to larger and sharper peaks with the best performing electrode using $16.7 \%$ Nafion. Further increasing Nafion content reduces the size of the peaks, but not their sharpness, suggesting that less catalyst is available, but ionic resistance in the electrode is still low. Figure 5-2 plots the platinum area calculated for each Nafion loading, including cells with 


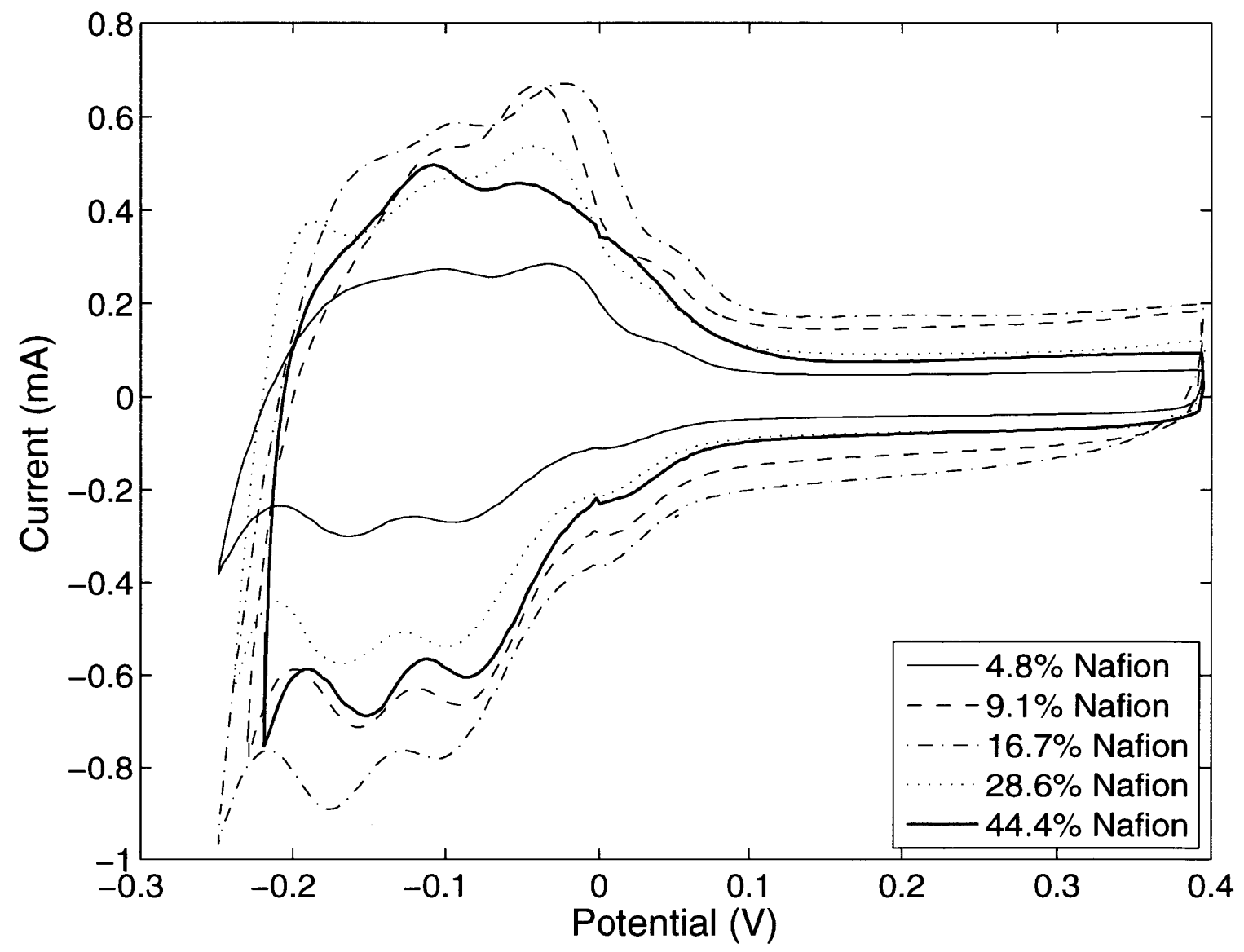

Figure 5-1: Voltagram of standard cells with varied Nafion content all with $0.3 \frac{\mathrm{mg}}{\mathrm{cm}^{2}}$ catalyst loading. 


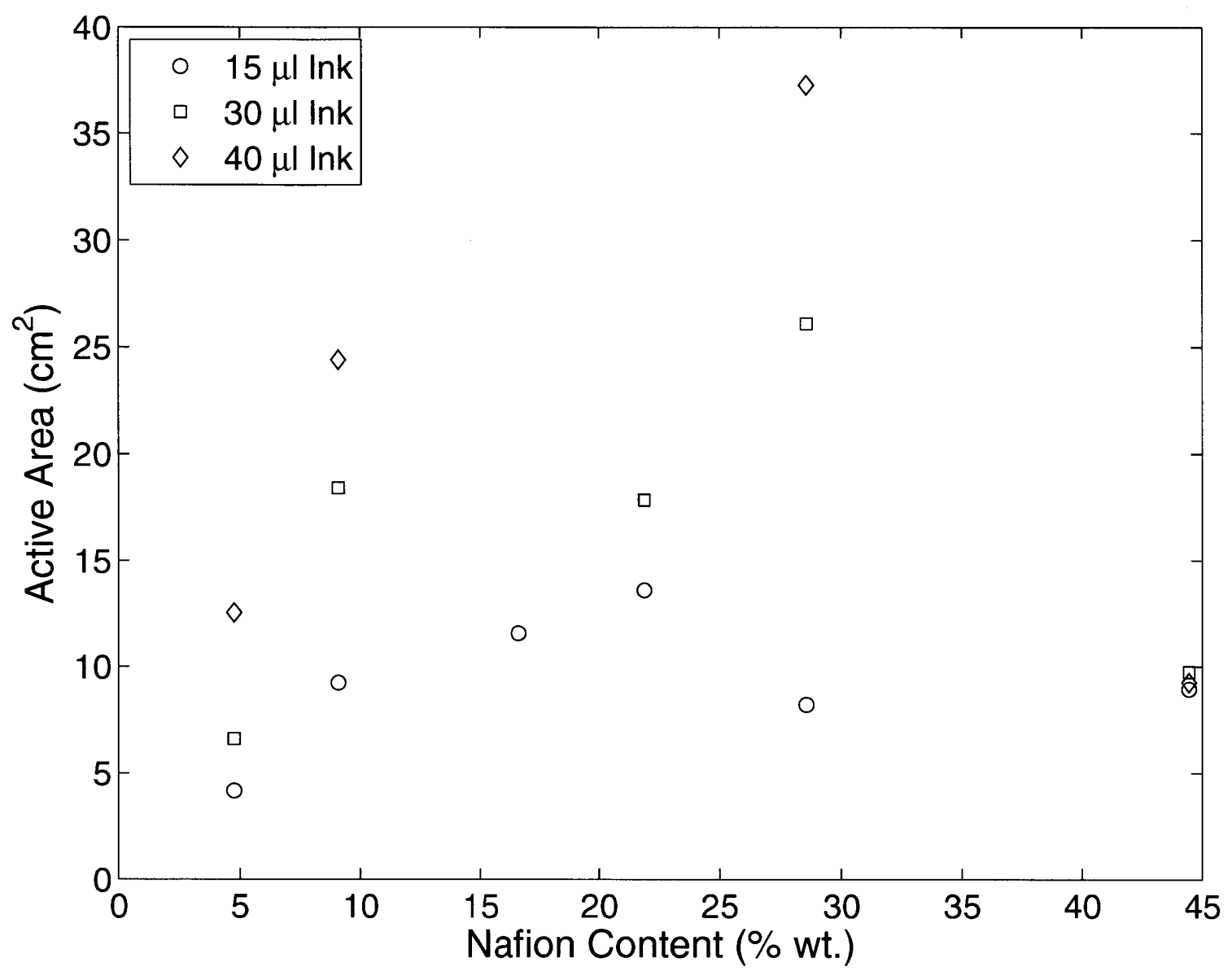

Figure 5-2: Plot of electrochemically active catalyst area as Nafion loading of the electrode is changed.

larger catalyst loadings. While $21.8 \%$ Nafion content performs the best at lower catalyst loadings, $28.6 \%$ Nafion content is consistently the best at larger catalyst loadings. Results in the literature [15] typically show that a Nafion content around $30 \%$ is the ideal loading for fuel cell electrodes and these measurements confirm those results.

\subsubsection{Impedance Spectroscopy with Changing Nafion Content}

Impedance spectroscopy provides a more detailed look at resistance in an electrode than cyclic voltammetry. As such, it can show both the intended impact of changes in Nafion loading, reduced ionic resistance, in addition to the unintended consequence of Nafion loading, blocked catalyst sites. To illustrate the ability of impedance spectroscopy to differentiate between electrodes with different Nafion contents, an impedance experiment was run with six cells: three with standard working electrodes and three with low-Nafion working electrodes. 


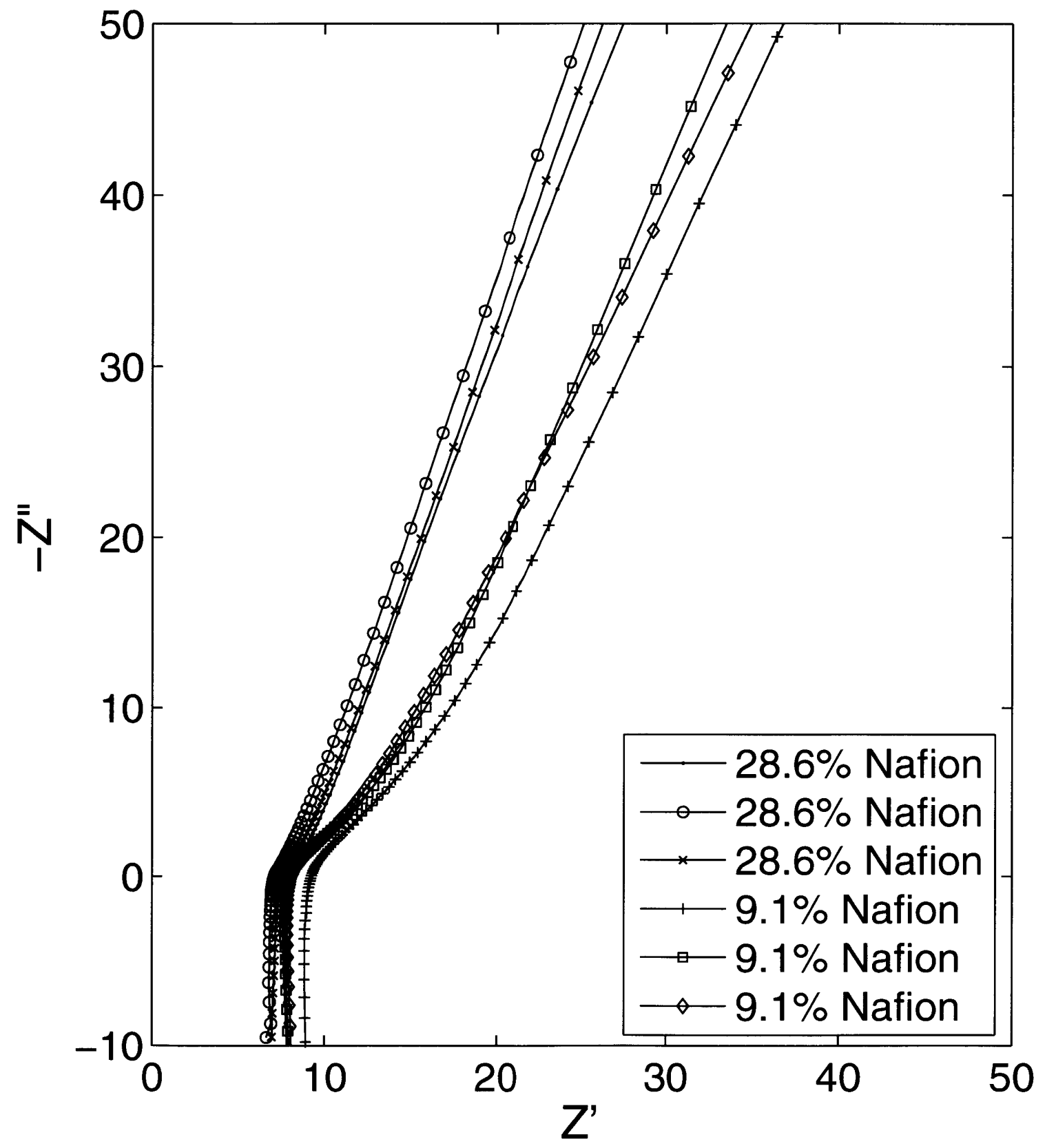

Figure 5-3: Nyquist plots measured using catalyzed carbon paper counter electrodes showing the influence of working electrode Nafion loading. 


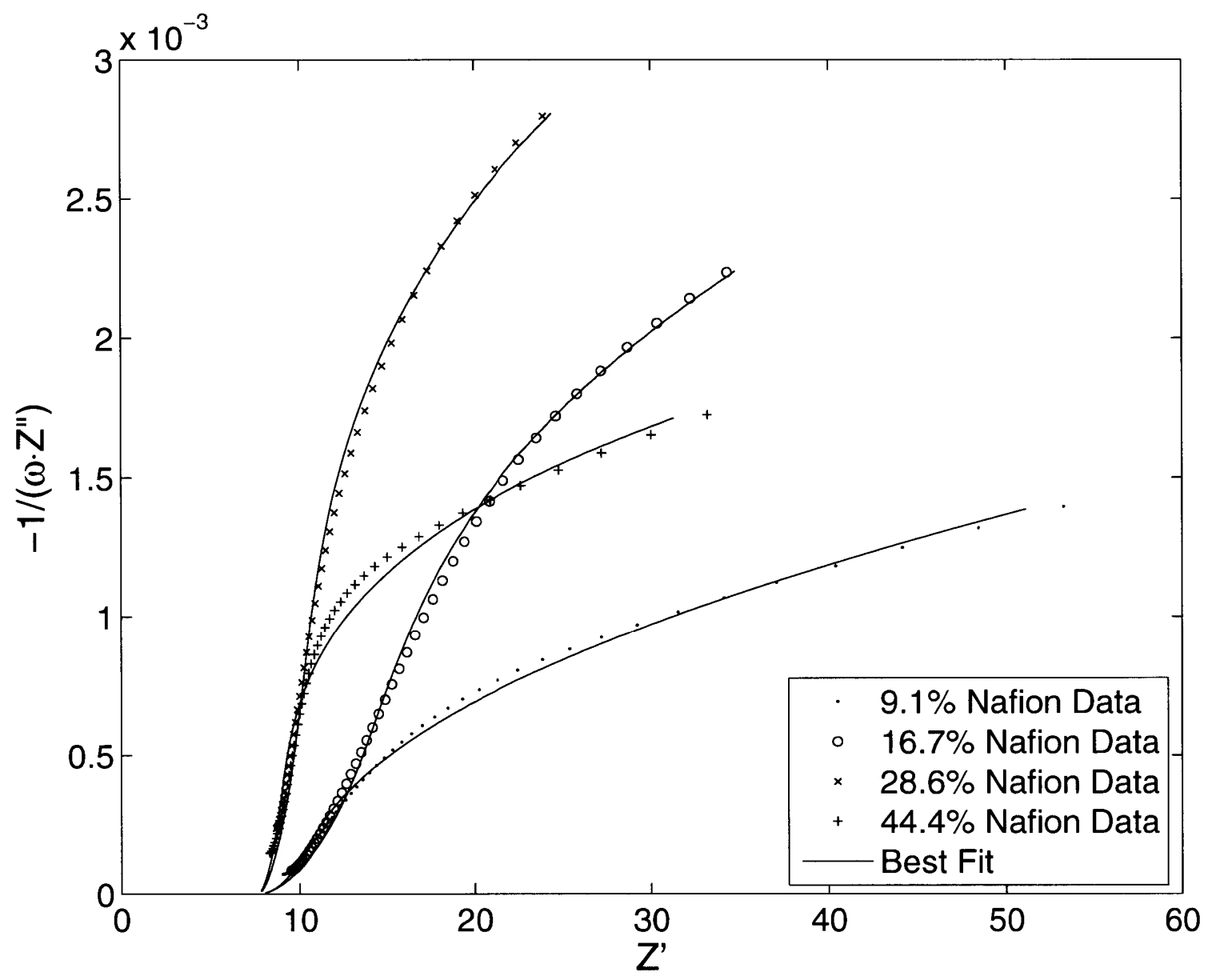

Figure 5-4: Capacitance plot of standard cells with changing Nafion content.

The Nyquist plot of these impedance spectra are shown in Figure 5-3. The electrodes group very clearly into two clusters based on the type of working electrode used. Additionally, the low-frequency asymptotes in the impedance spectra of the low-Nafion electrodes show a clear offset, relative to the standard cells, along the real impedance axis. This corresponds well to the predicted effect that ionic resistance in the electrode has on impedance based on the transmission line models presented in Chapter 3.

As seen in Figure 5-1, the ideal Nafion content in a fuel cell electrode can be determined using cyclic voltammetry to measure the electrochemically active catalyst area. Catalyst in electrodes with too little Nafion is underutilized, because some particles are not connected to the Nafion matrix. In electrodes with too much Nafion, the Nafion coats the catalyst so thickly that reactants and products cannot get to or from the catalyst surface. The existence of this ideal Nafion content can also be found using impedance spectroscopy. Figure 5-4 
shows a capacitance plot of four standard electrodes that use ink with increasing Nafion content. In the figure we see that the high-frequency slope of the plot increases steadily with Nafion content. The low-frequency asymptotic value, which correlates well to catalyst availability, shows the importance of using the proper amount of Nafion. The asymptotic value rises as the Nafion content is increased from $9.1 \%$ to $16.7 \%$ to $28.6 \%$. However, when the Nafion content is further increased to $44.4 \%$, the asymptotic values drops even below the value for $16.7 \%$ Nafion. This suggests that $28.6 \%$ Nafion content is close to the optimum amount of Nafion, which matches with the previous cyclic voltammetry experiments, shown in Figures 5-1 and 5-2, and with published results in the literature [15].

\subsection{Influence of Catalyst Loading on Ionic Resistance}

The methanol oxidation reaction that takes place at the DMFC anode is a complex and fairly slow reaction [7] which requires a large overpotential. A typical method used to speed up such reactions while reducing the need to run larger overpotentials is to add more catalyst area to the electrode. As addressed in Chapter 2, adding more catalyst to a DMFC anode will eventually cause electrode performance to decrease. The Axiomatic Design analysis presented in Section 3.1.2 shows that this observed catalyst loading limit is a result of coupling between the catalyst loading function and the ionic resistance functions of the fuel cell electrode. This section explores the impact of catalyst loading on the performance of electrodes using cyclic voltammetry and impedance spectroscopy.

\subsubsection{Effect of Catalyst Loading with Cyclic Voltammetry}

In Section 4.1.2, cyclic voltammetry is shown to be capable of qualitatively assessing the impact of ionic resistance on the performance of an electrode by measuring changes in available catalyst area and changes in the shape of the hydrogen adsorption peaks. Figure 55 shows the cyclic voltagrams of a series of $9.1 \%$ Nafion electrodes made with increasing amounts of ink and, therefore, increasing catalyst loading. The low-Nafion ink, $9.1 \%$ Nafion, is used in place of the ideal Nafion content, $28.6 \%$ Nafion, because the catalyst limit should appear at much lower catalyst loadings making the experiment easier. Clearly, the hydrogen adsorption peaks are increasing with increasing catalyst loading and there does not appear to be a limit to catalyst loading in this case. What is not clear, however, is whether or not 


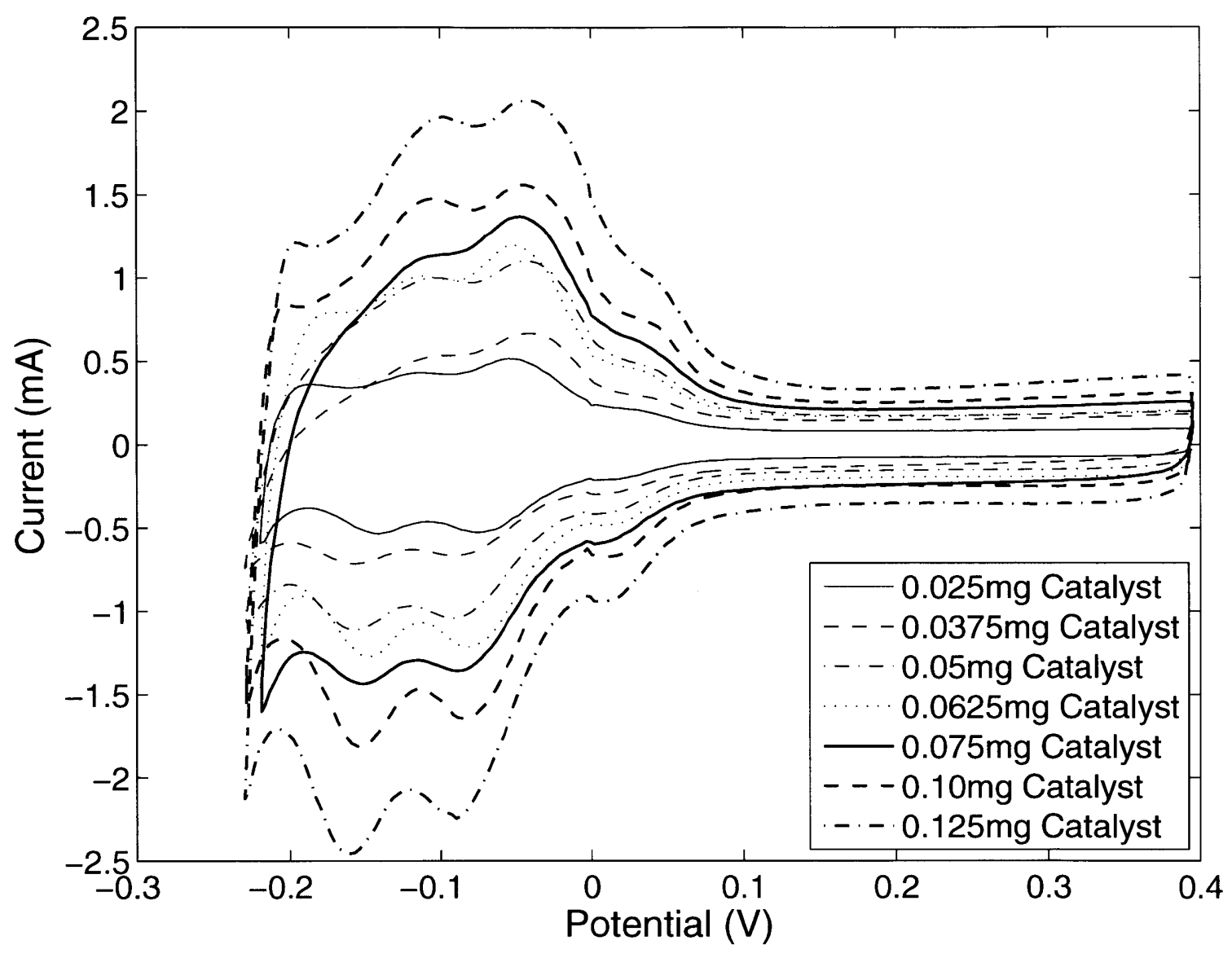

Figure 5-5: Voltagram of standard cells with varied catalyst loading 


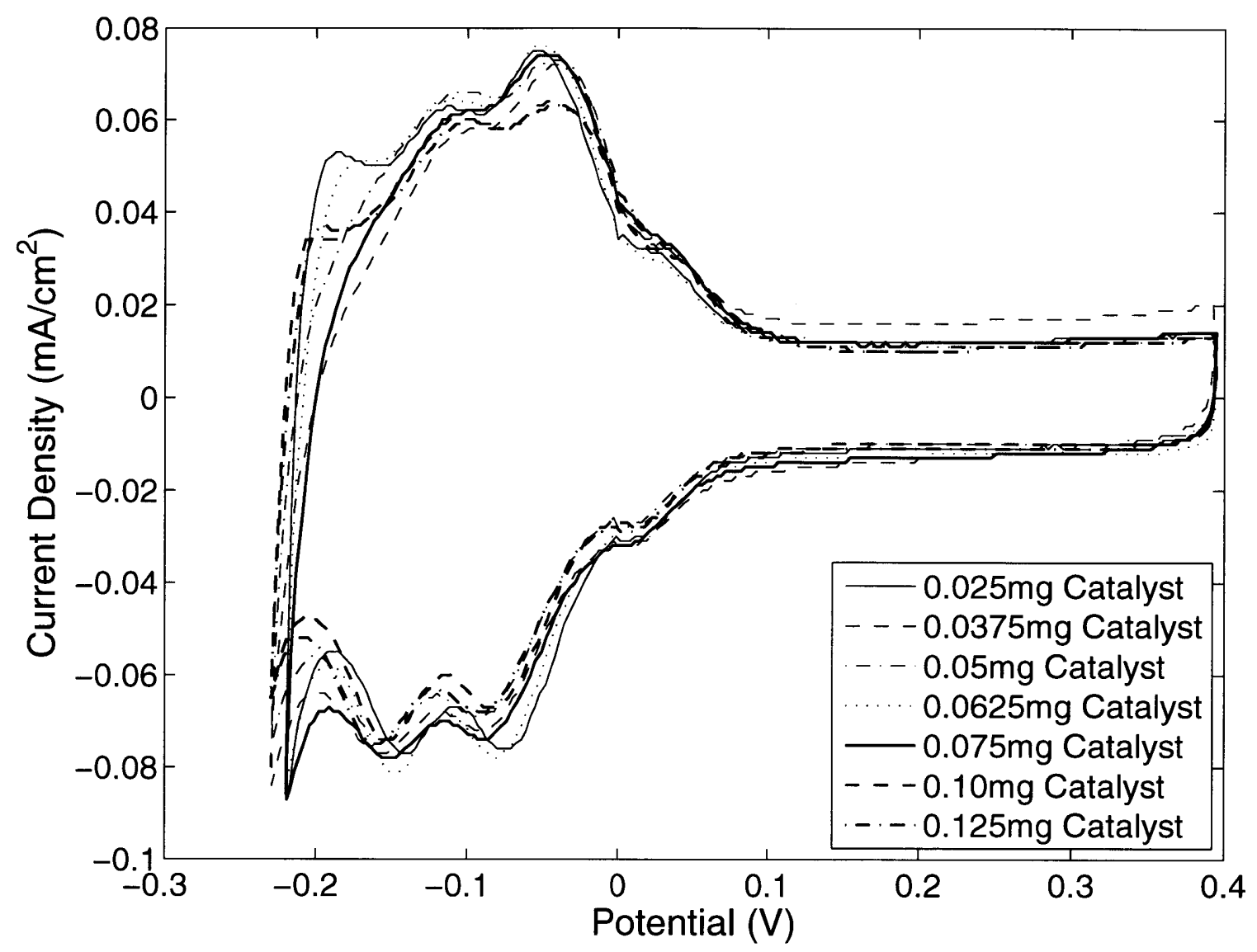

Figure 5-6: Voltagram of standard cells with varied catalyst loading that is normalized to measured active platinum area.

the shapes of the adsorption peaks are changing with catalyst loading, which would indicate a change in the ionic resistance behavior with catalyst loading. To investigate this question, the cyclic voltagrams in Figure 5-5 are normalized to their measured active catalyst area and replotted in Figure 5-6. With the current divided by the available catalyst area, the electrodes at all catalyst loadings seem to have identical behavior, suggesting that even when large amounts of catalyst are applied, there is little to no change in ionic resistance in the electrode.

The lack of any diminishing returns on catalyst in the electrodes is made very clear by plotting the measured active catalyst area, as in Figure 5-7. In this plot, the active catalyst area increases linearly with catalyst loading. This plot also underscores the position of the $28.6 \%$ Nafion electrodes as the ideal Nafion loading cells since, for all but one case, they have the largest active catalyst area. The slopes of these lines give the specific active catalyst area for each electrode and are shown in Table 5.1. The value for the ideal Nafion loading 


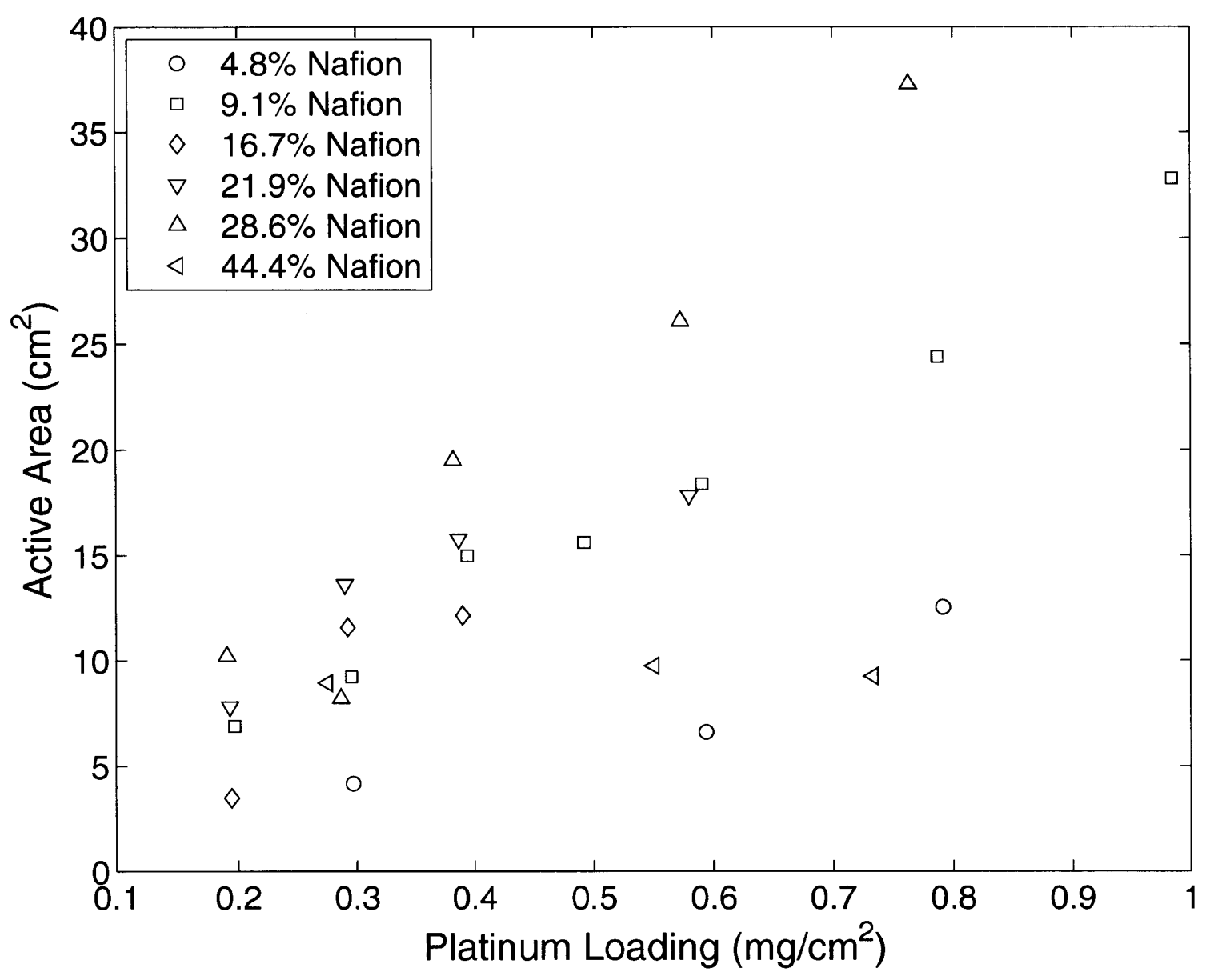

Figure 5-7: Plot of electrochemically active catalyst area as catalyst loading in the electrode is changed.

of $40.2 \frac{\mathrm{m}^{2}}{\mathrm{~g}}$ is only slightly lower than the typical range expected for commercial catalyst powders of $50-100 \frac{\mathrm{m}^{2}}{\mathrm{~g}}$, suggesting that the catalyst utilization of these electrodes is around $50 \%$ or greater, depending on the actual specific surface area of the catalyst used, which was not measured. The one ink that did not seem to improve was the $44.4 \%$ Nafion ink which returned roughly the same active area no matter the catalyst loading. This is most likely a result of the excessive Nafion in the electrode blocking access to catalyst beyond a certain point.

Table 5.1: Available catalyst area per mass of catalyst applied.

\begin{tabular}{|c|c|c|c|c|c|}
\hline Electrode Nafion Content & $4.8 \%$ & $9.1 \%$ & $16.7 \%$ & $21.9 \%$ & $28.6 \%$ \\
\hline \hline Specific Active Catalyst Area $\left(\mathrm{m}^{2} / \mathrm{g}\right)$ & 12.9 & 25.9 & 25.4 & 28.7 & 40.2 \\
\hline
\end{tabular}




\subsubsection{Catalyst Loading Changes in Impedance Spectroscopy}

Cyclic voltammetry, in this case, is not sufficient to see the catalyst loading limit imposed on fuel cell electrodes by the ionic resistance in the electrodes. Impedance spectroscopy, however, is well suited to measuring the effects of ionic resistance and is used to investigate the effect of catalyst loading on electrode ionic resistance. The impedance spectra of four cells are shown in Figure 5-8. Each cell has a different combination of catalyst loading, either 20 or $60 \mu \mathrm{L}$ of ink applied, and electrode Nafion content, either standard or low-Nafion ink. As in Figure 5-3, changing from standard Nafion loading to low-Nafion loading ink appears in the low-frequency asymptote region as a shift along the positive real impedance axis. Similarly, adding catalyst to a cell by going from $20 \mu \mathrm{L}$ of applied ink to $60 \mu \mathrm{L}$ yields a rightward shift of the low-frequency asymptote for both types of ink though the shift is less pronounced with the low-Nafion ink. The real axis shift is expected for both inks, since the thicker catalyst layer will result in a longer ionic conductive pathway and therefore a larger overall real impedance.

The addition of catalyst, however, seems to do more than the simple shift seen when changing Nafion content of the electrode. In the case of the standard Nafion content ink, the addition of catalyst results in a rightward shift in the Nyquist plot, but the overall magnitude of the impedance is lower at all points in the low-frequency asymptote. This can be seen most clearly at the end of the low-frequency asymptotes where the cell with more catalyst is clearly closer to the origin than the lower catalyst cell. This is an indicator that the cell with more catalyst has more catalyst area available and therefore a lower impedance. This trend, however, does not hold so clearly for the cells using the low-Nafion content ink. The endpoints of the low-frequency asymptotes appear to have roughly the same magnitude for both the high and low catalyst loading cases. This observation implies that despite the additional catalyst present in the electrode, very little of the additional catalyst area is utilized.

The effect that Nafion loading has on catalyst accessibility can be more clearly seen if these impedance spectra are plotted on a capacitance plot, as in Figure 5-9. In this plot, the horizontal asymptote represents the area of catalyst electrochemically available in the electrode and the initial slope of the plot corresponds to the electrode's intrinsic ionic resistance. This plot clarifies the influence of both catalyst loading and Nafion content. 


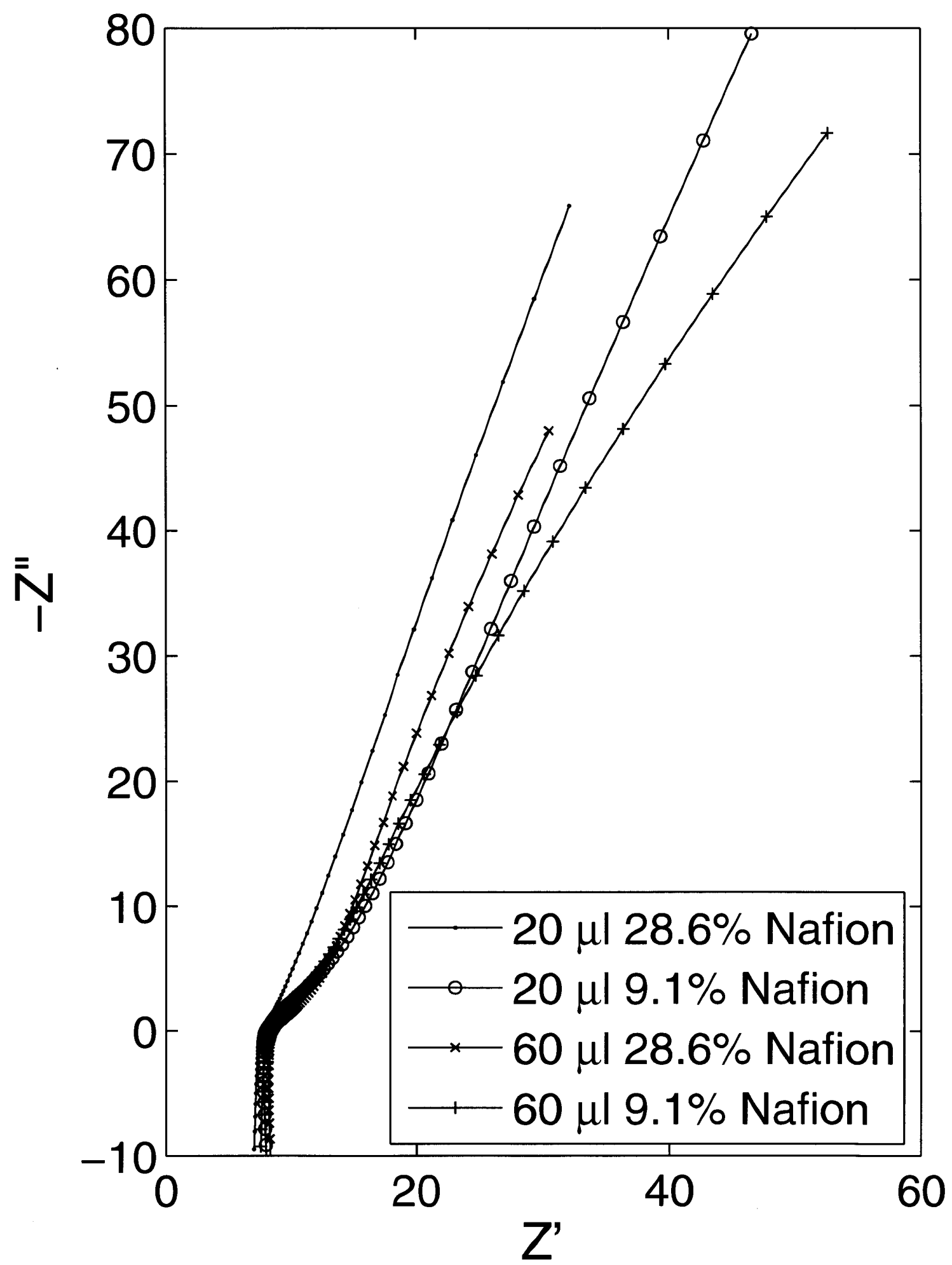

Figure 5-8: Nyquist plots of cells with high and low Nafion content and high and low catalyst content. 


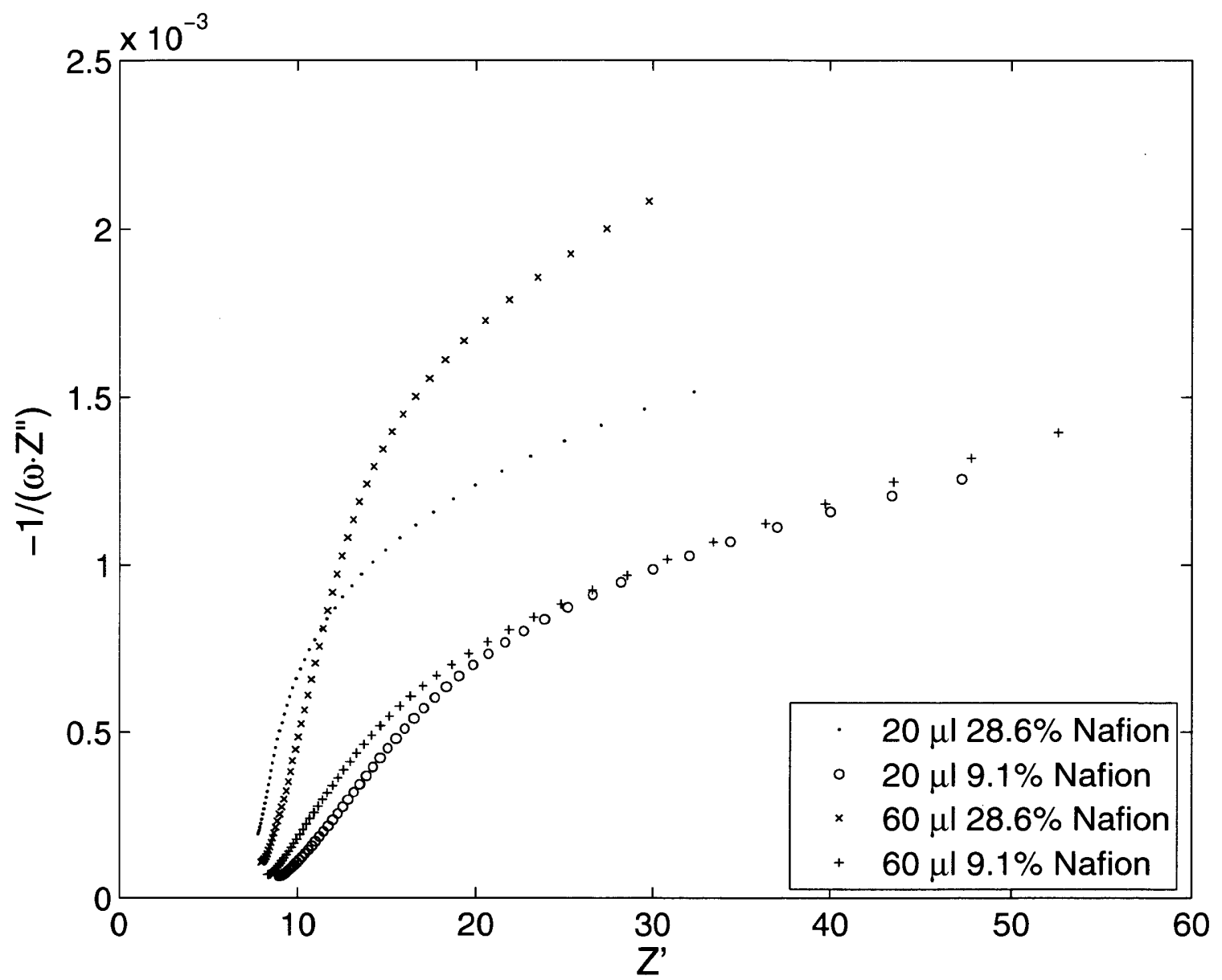

Figure 5-9: Capacitance plot of cells with high and low Nafion content and high and low catalyst content. 
Table 5.2: Best fit parameters for low-Nafion electrodes using the modified transmission line model.

\begin{tabular}{|l||c|c|c|c|}
\cline { 2 - 5 } \multicolumn{1}{c|}{} & $R_{s}$ & $R$ & $T$ & $\phi$ \\
\hline \hline $20 \mu l$ Standard Ink & 8.924 & 2.359 & 0.0018 & 0.842 \\
\hline $40 \mu l$ Standard Ink & 7.796 & 3.006 & 0.0029 & 0.793 \\
\hline $60 \mu l$ Standard Ink & 7.683 & 9.608 & 0.0034 & 0.760 \\
\hline $80 \mu l$ Standard Ink & 9.456 & 2.464 & 0.0028 & 0.747 \\
\hline
\end{tabular}

For instance, the initial slopes of the low-Nafion cells match with one another and are significantly shallower than the initial slopes of the standard Nafion content cells. From the horizontal asymptotes we see that the two electrodes using the low-Nafion ink have approximately the same electrochemically active catalyst area. This available area is less than that of the $20 \mu \mathrm{L}$ cell with standard ink simply as a result of more Nafion in the electrode and much less than the $60 \mu \mathrm{L}$ cell with standard ink.

\subsubsection{Catalyst Loading Limit}

Unlike the cyclic voltammetry experiments, impedance spectroscopy of standard electrodes can be used to find the limit at which additional catalyst begins to degrade cell performance. To illustrate this point, electrodes were created using standard Nafion loading with progressively more ink and therefore more catalyst. The capacitance plots of the resulting impedance spectra are shown in Figure 5-10. The two middle-loading cells show a decidedly larger low-frequency asymptote than the electrode with the least, or that with the most ink. Using the best fit of a modified transmission line, the anticipated trend is clearly present in the capacitive term, $T$. The best fit parameters for these electrodes are shown in Table 5.2. The capacitive parameter increases to a maximum value at an ink loading of $60 \mu \mathrm{L}$, before decreasing as ink loading reaches $80 \mu \mathrm{L}$. Figure 5-11 shows the value of the capacitive term as a function of ink loading. This indicates that the impedance spectroscopy experiment is capable of seeing the deleterious effects of too much catalyst, unlike the cyclic voltammetry experiments.

Performing a similar experiment, but with low-Nafion electrodes, yields a similar result. Figure 5-12 shows the capacitance plots for low-Nafion electrodes as the catalyst loading is increased from $20 \mu \mathrm{L}$ to $60 \mu \mathrm{L}$. Again there is a maximum performance before additional catalyst begins to impair catalyst availability. In this case, the maximum performance is 


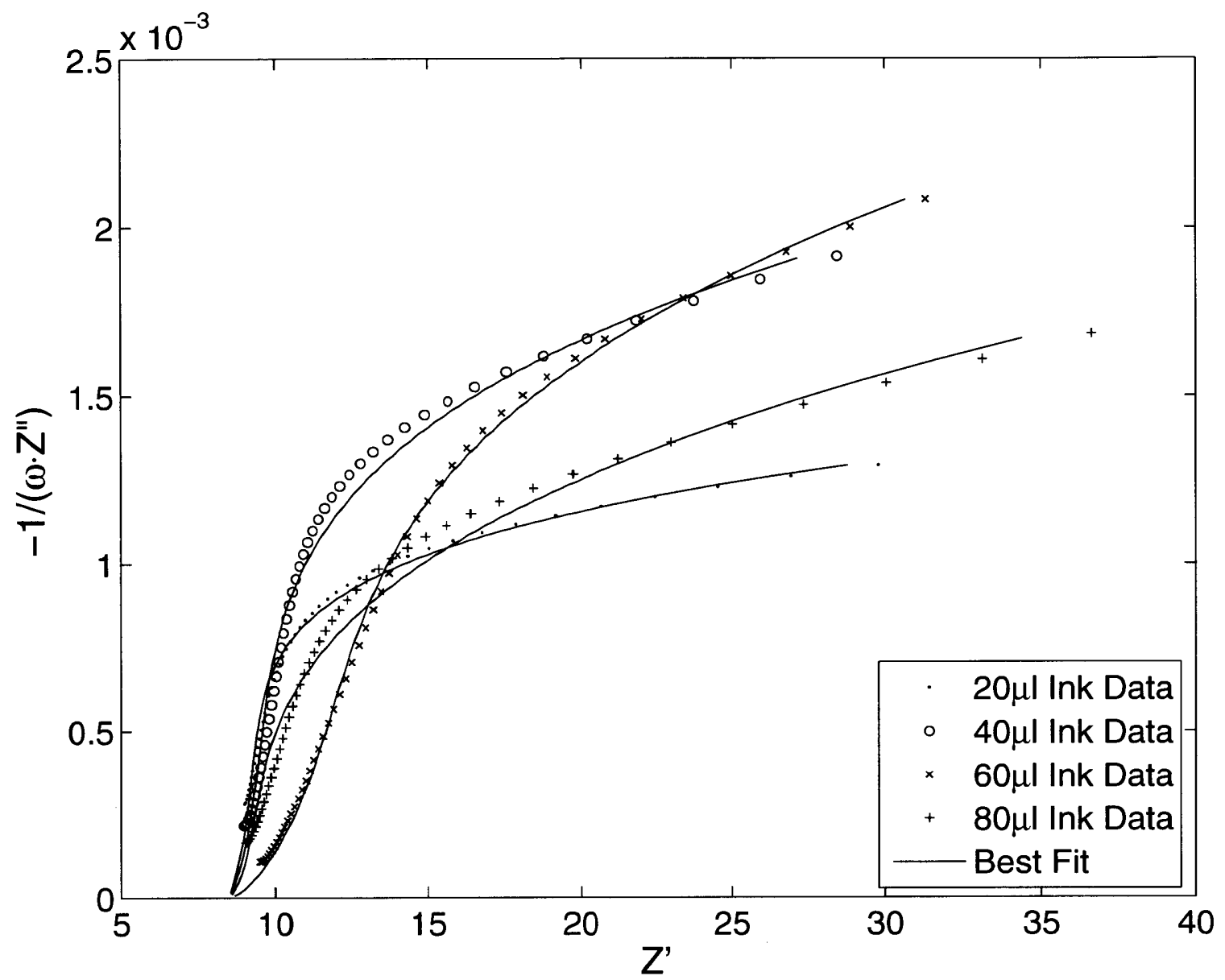

Figure 5-10: Capacitance plot of standard cells as catalyst loading is increased. 


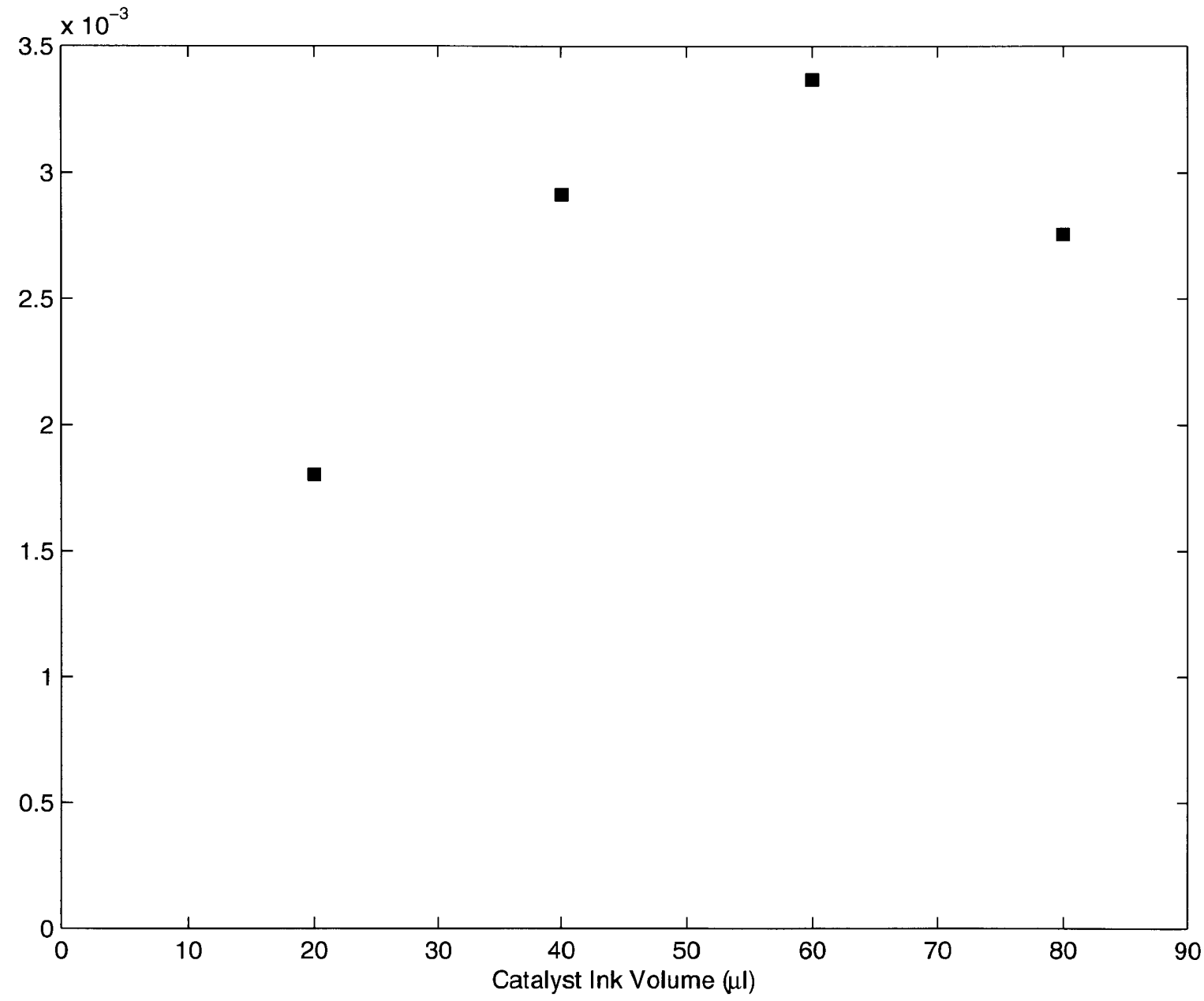

Figure 5-11: Best fit capacitance as a function of catalyst loading for standard ink composition. 


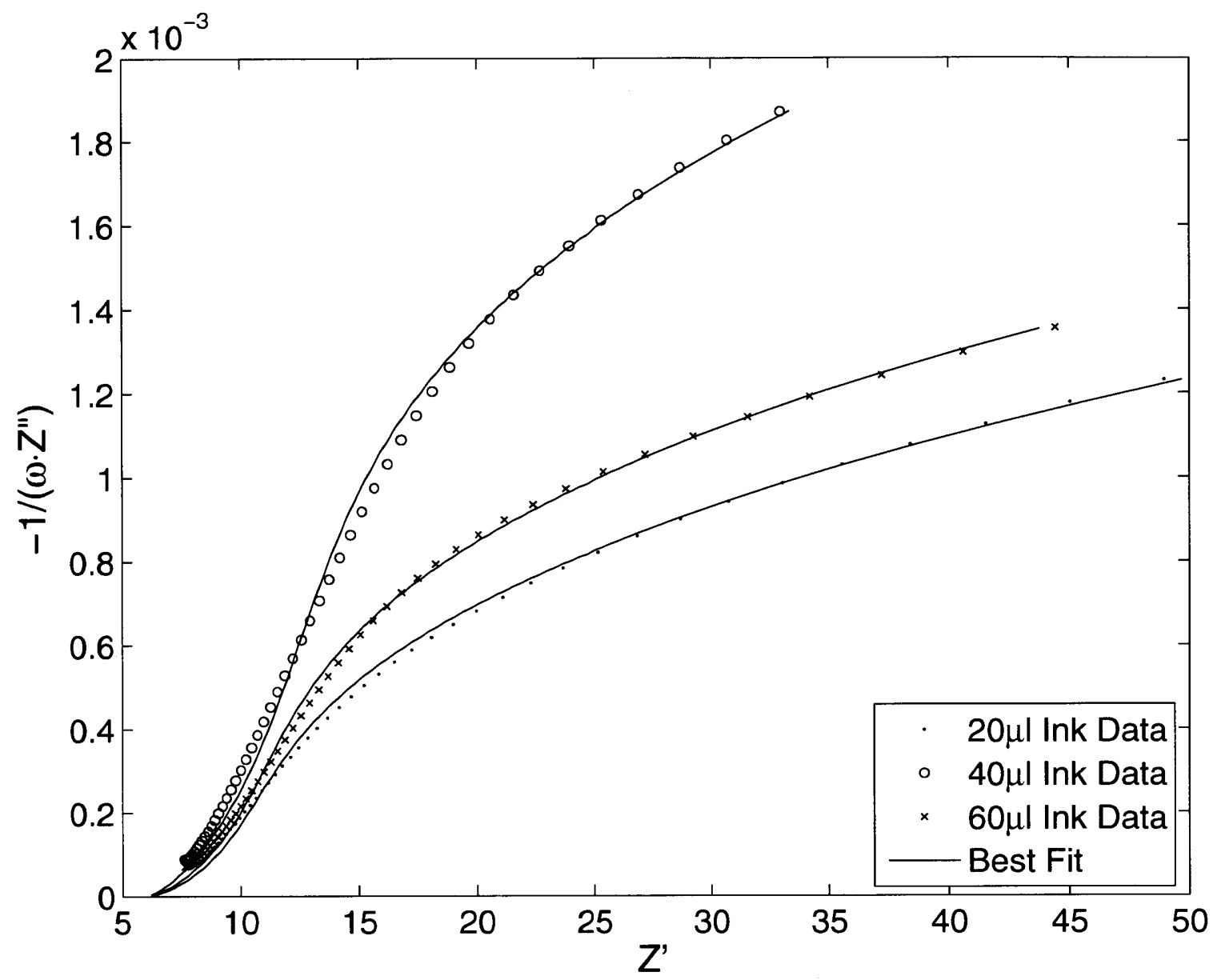

Figure 5-12: Capacitance plot of standard cells with low-Nafion ink as catalyst loading is increased.

at $40 \mu \mathrm{L}$ ink loading, as opposed to $60 \mu \mathrm{L}$ with the standard Nafion content. It is expected that a reduction in Nafion in the electrode would lead to a reduction in the ideal catalyst loading, since reduced Nafion increases ionic resistance in the electrode, thus making the electrode more sensitive to thickness. The fit parameters for these electrodes are shown in Table 5.3. The capacitive term is plotted as a function of catalyst loading in Figure 5-13, which confirms the interpretation of the plot that there is a maximum catalyst available at $60 \mu \mathrm{L}$.

\subsection{Performance of Structured Cells}

Cyclic voltammetry was initially used to investigate the performance of structured cells, but difficulty fabricating these cells made meaningful results impossible to ascertain. The only 
Table 5.3: Best fit parameters for Low-Nafion electrodes using the modified transmission line model.

\begin{tabular}{|c||c|c|c|c|}
\cline { 2 - 5 } \multicolumn{1}{c|}{} & $R_{s}$ & $R$ & $T$ & $\phi$ \\
\hline \hline $20 \mu l$ Low-Nafion Ink & 6.413 & 12.940 & 0.0022 & 0.712 \\
\hline $40 \mu l$ Low-Nafion Ink & 5.329 & 18.413 & 0.0030 & 0.757 \\
\hline $60 \mu l$ Low-Nafion Ink & 5.877 & 14.141 & 0.0023 & 0.731 \\
\hline
\end{tabular}

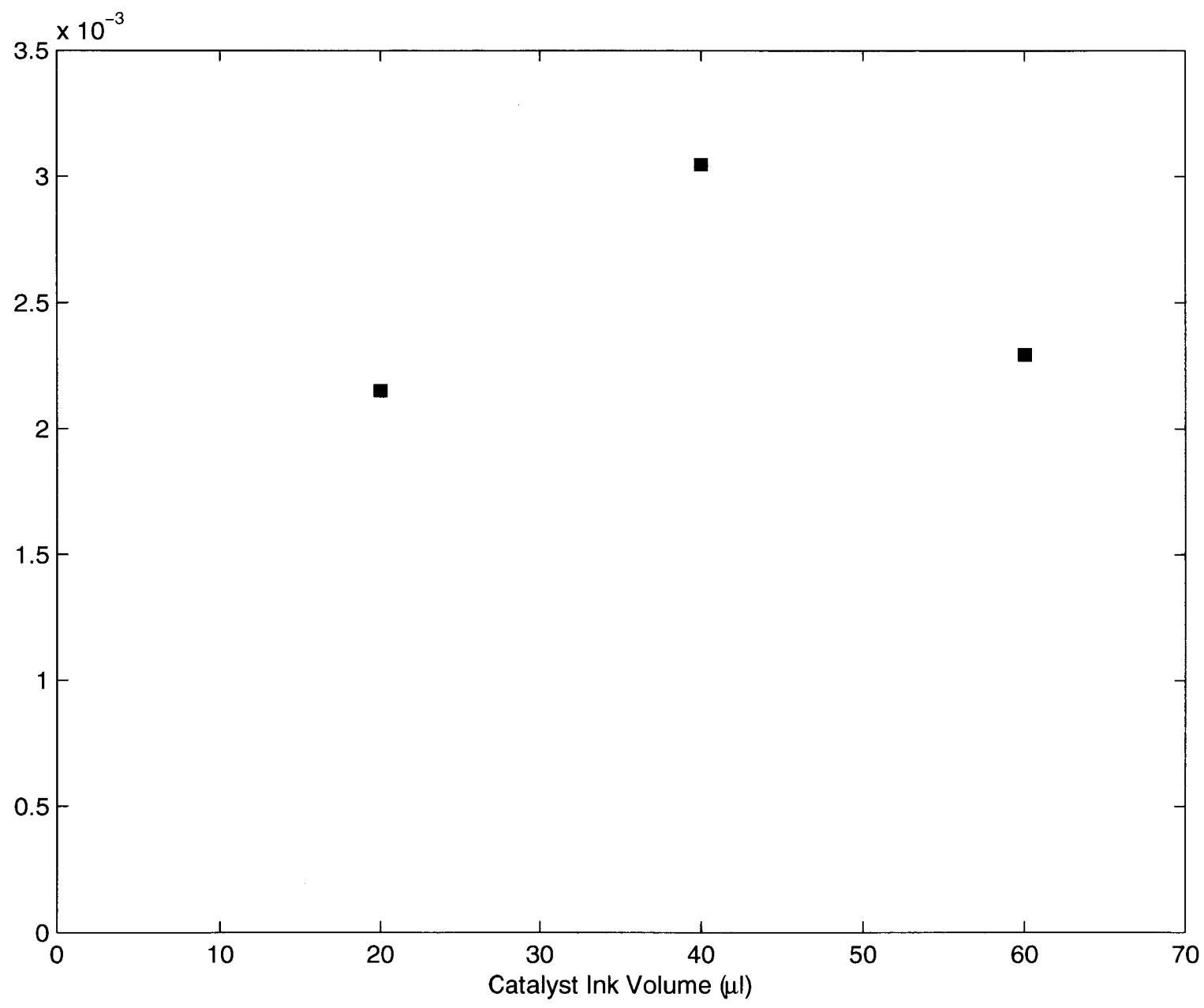

Figure 5-13: Best fit capacitance as a function of catalyst loading for standard ink composition. 


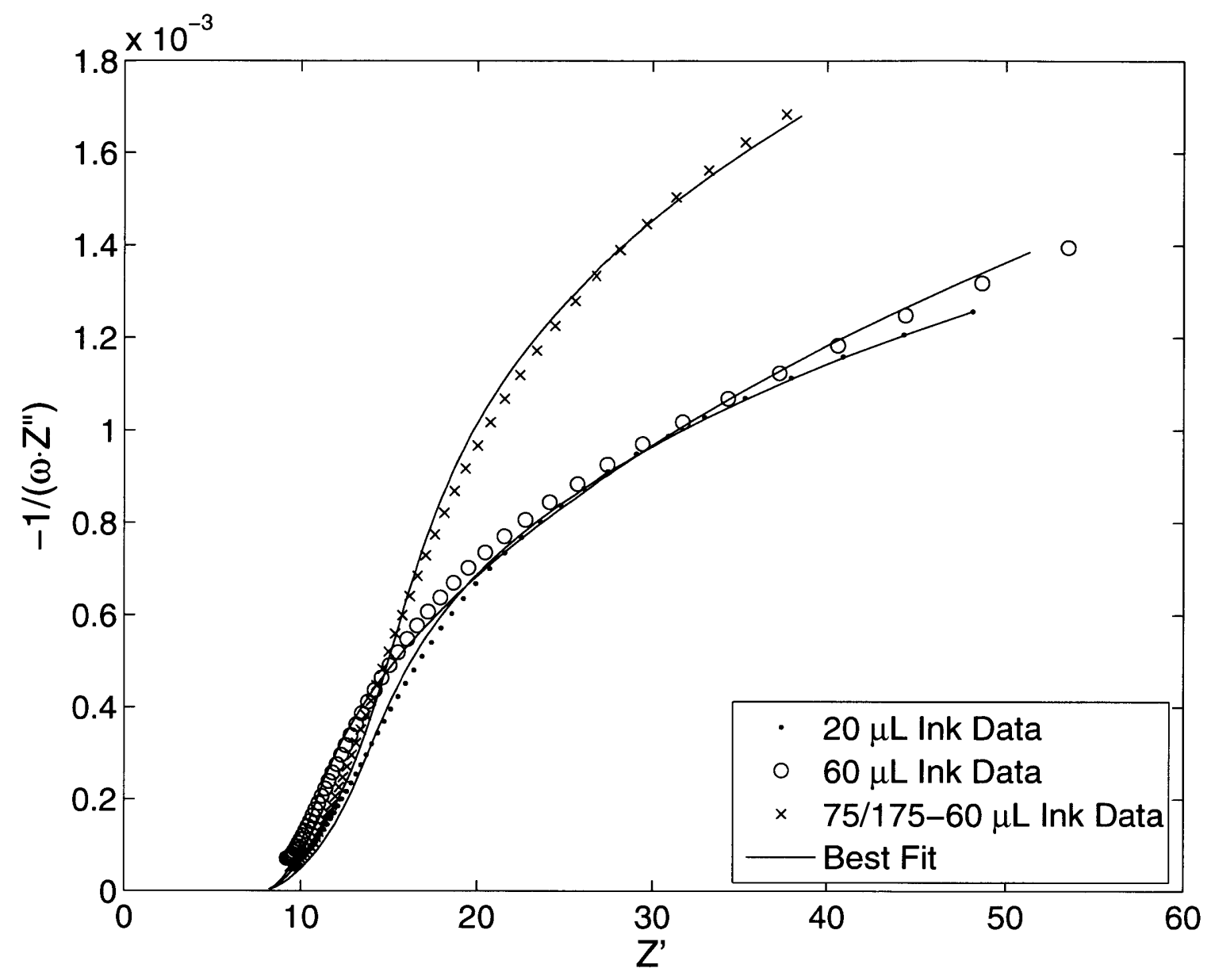

Figure 5-14: Capacitance plot comparing structured and standard electrodes created with $9.1 \%$ Nafion content.

fabrication option capable of producing structured cells on glassy carbon supports was to cast the Nafion posts onto a piece of carbon paper, then punch out a circular section and attach it to the glassy carbon electrode support with a small amount of Nafion solution. The details of this process are described in Section 4.4.2.2. This fabrication method resulted in cells with large double-layer capacitance and large contact resistance, which made it impossible to extract meaningful conclusions about the structured electrodes fabricated in this fashion using cyclic voltammetry. A more detailed explanation of the cyclic voltammetry results for structured electrodes can be found in Section B.1 of the Appendix.

A capacitance plot of the impedance data for standard and structured electrodes made with $9.1 \%$ Nafion content is shown in Figure 5-14. The structured cells have $75 \mu$ m diameter Nafion posts with center-to-center spacing of $175 \mu \mathrm{m}$ and are labeled "75/175." For the electrodes with low levels of Nafion, the capacitance plot shows almost no additional catalyst 


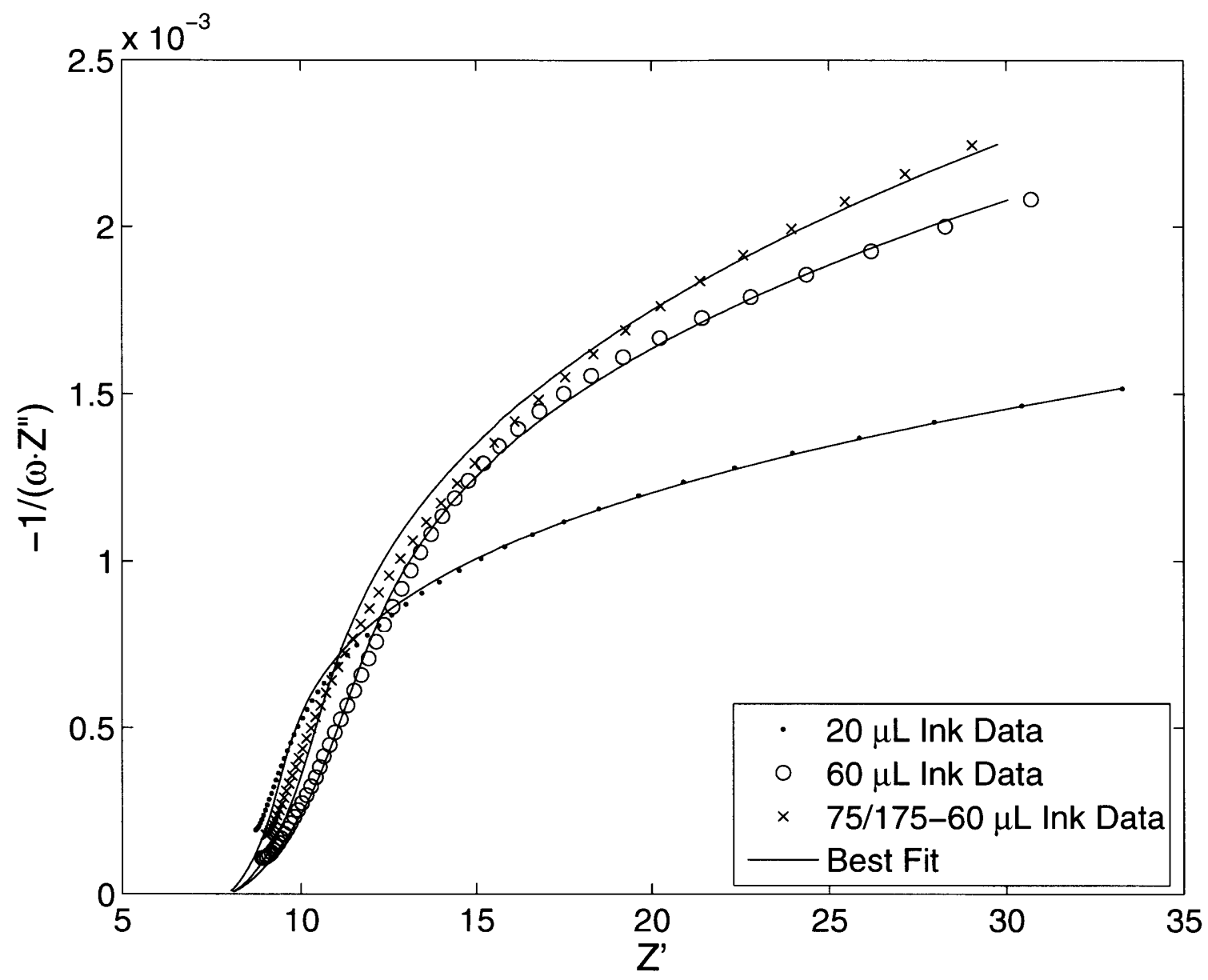

Figure 5-15: Capacitance plot comparing structured and standard electrodes created with $28.6 \%$ Nafion content.

available when changing from an ink loading of $20 \mu \mathrm{L}$ to an ink loading of $60 \mu \mathrm{L}$. Despite the tripling of the catalyst in the electrode, barely any of this investment is returned in the form of electrochemically available catalyst area. This is the expected result, since the low Nafion content of the ink should lead to fewer catalyst particles ionically connected to the membrane through the Nafion matrix. Furthermore, the large ionic resistance within the low-Nafion electrode will make the additional catalyst far from the membrane more difficult to reach and thus less utilized. The addition of Nafion posts through the electrode thickness results in a large jump in the asymptotic capacitance value when using $60 \mu \mathrm{L}$ of low-Nafion ink. This indicates that the ionically conductive channels allow the additional catalyst in the ink to be resistively closer to the membrane, even though the catalyst particles are physically farther from the membrane.

For the electrodes with a standard Nafion loading, shown in Figure 5-15, moving from 


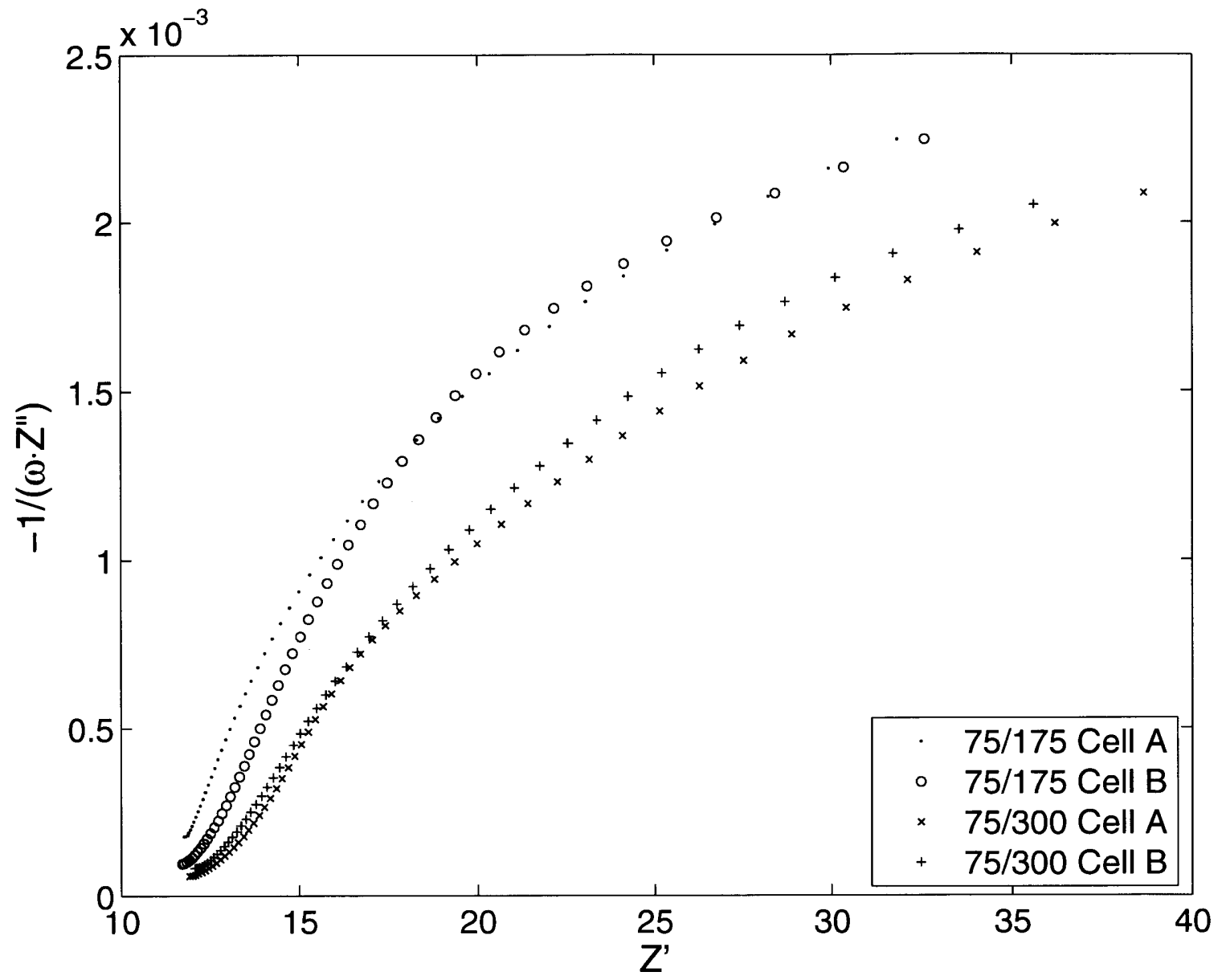

Figure 5-16: Capacitance plot of structured electrodes with standard ink.

$20 \mu \mathrm{L}$ of ink to $60 \mu \mathrm{L}$ of ink results in a clear increase in the catalyst available as seen in the asymptotic capacitance value. Again, adding ionically conducting posts to the electrodes with $60 \mu \mathrm{L}$ of ink results in a noticeable increase in catalyst utilization. The Nafion posts have a much larger influence on the low-Nafion content ink than on the standard Nafion content ink. This is expected, as the standard content ink should provide better catalyst utilization than the low-Nafion ink as the catalyst loading increases.

Figure 5-16 shows the capacitance plots of two types of structured electrodes, both made with $60 \mu \mathrm{L}$ of standard ink. The first two cells have $75 \mu \mathrm{m}$ diameter Nafion posts with $175 \mu \mathrm{m}$ center-to-center post spacing and are labeled "75/175." The second set of cells also have $75 \mu \mathrm{m}$ diameter posts, but have a center-to-center spacing of $300 \mu \mathrm{m}$ and are labeled "75/300." The group of cells with the closer post packing show significantly more catalyst area available than the sparse posts, with a roughly similar initial slope indicating 
Table 5.4: Average best fit parameters for structured and standard cells with $60 \mu \mathrm{L}$ standard ink using a modified transmission line model.

\begin{tabular}{|cc|c|c|c|c|}
\cline { 3 - 6 } \multicolumn{1}{c|}{} & $R_{s}$ & $R$ & $T$ & $\phi$ \\
\hline \hline \multirow{2}{*}{$75 / 175$} & Mean & 9.237 & 8.943 & 0.0037 & 0.745 \\
\cline { 2 - 6 } & St. Dev. & 0.220 & 2.7872 & $7.2 \cdot 10^{-5}$ & 0.014 \\
\hline $75 / 300$ & Mean & 12.046 & 12.7211 & 0.0036 & 0.717 \\
\cline { 2 - 6 } & St. Dev. & 1.649 & 2.874 & $1.99 \cdot 10^{-4}$ & 0.0244 \\
\hline \multirow{2}{*}{ Std. } & Mean & 8.044 & 16.6 & 0.0035 & 0.72 \\
\cline { 2 - 6 } & St. Dev. & 2.2143 & 9.79 & 0.0007 & 0.036 \\
\hline
\end{tabular}

slightly better ionic conductivity. Fitting these data with the modified transmission line confirms this interpretation. The resulting average fit parameters from these two structured cells and the corresponding non-structured cell are shown in Table 5.4. A capacitance plot of the impedance spectra, based on the best-fit parameters, is shown in Figure 5-17. Both structured cells show a reduction in ionic conductivity and slight increases in the capacitancelike term indicating more available catalyst. The $75 / 175$ structured cells result in a $5.7 \%$ increase in available catalyst area. Using the parameters from Easton and Pickup [39] to translate capacitance into available catalyst mass results in the cell having about $0.1 \mathrm{mg}$ of available catalyst from the $0.15 \mathrm{mg}$ applied for a $66 \%$ catalyst utilization.

The performance change is even more striking with electrodes made with low-Nafion ink. Impedance spectra calculated from the average best fit of structured and unstructured cells are shown in Figure 5-18. The structured cells both show significantly larger lowfrequency asymptotes than the standard cell with equal loading. Interestingly, the order of the two types of structure is inverted in this case. That is, the electrode with more densely packed posts, $175 \mu \mathrm{m}$ center-to-center spacing, performs slightly worse that the more sparse structure with $300 \mu \mathrm{m}$ center-to-center spacing, whereas the opposite was true for the cells with standard ink. The reasons for this are unclear, but it is likely that the ideal structure parameters are dependent on the ink mixture used. From the modeling described in Chapter 3, it is clear that adding posts has a stronger influence on conductivity than changes in the structure geometry. A more advanced model or a more exhaustive experimental survey of the influence of structure geometry will be useful in determining the best structure for a particular electrode loading.

The average parameters returned from fitting these cells with the modified transmission line are shown in Table 5.5. These values also confirm the interpretation of the capacitance 


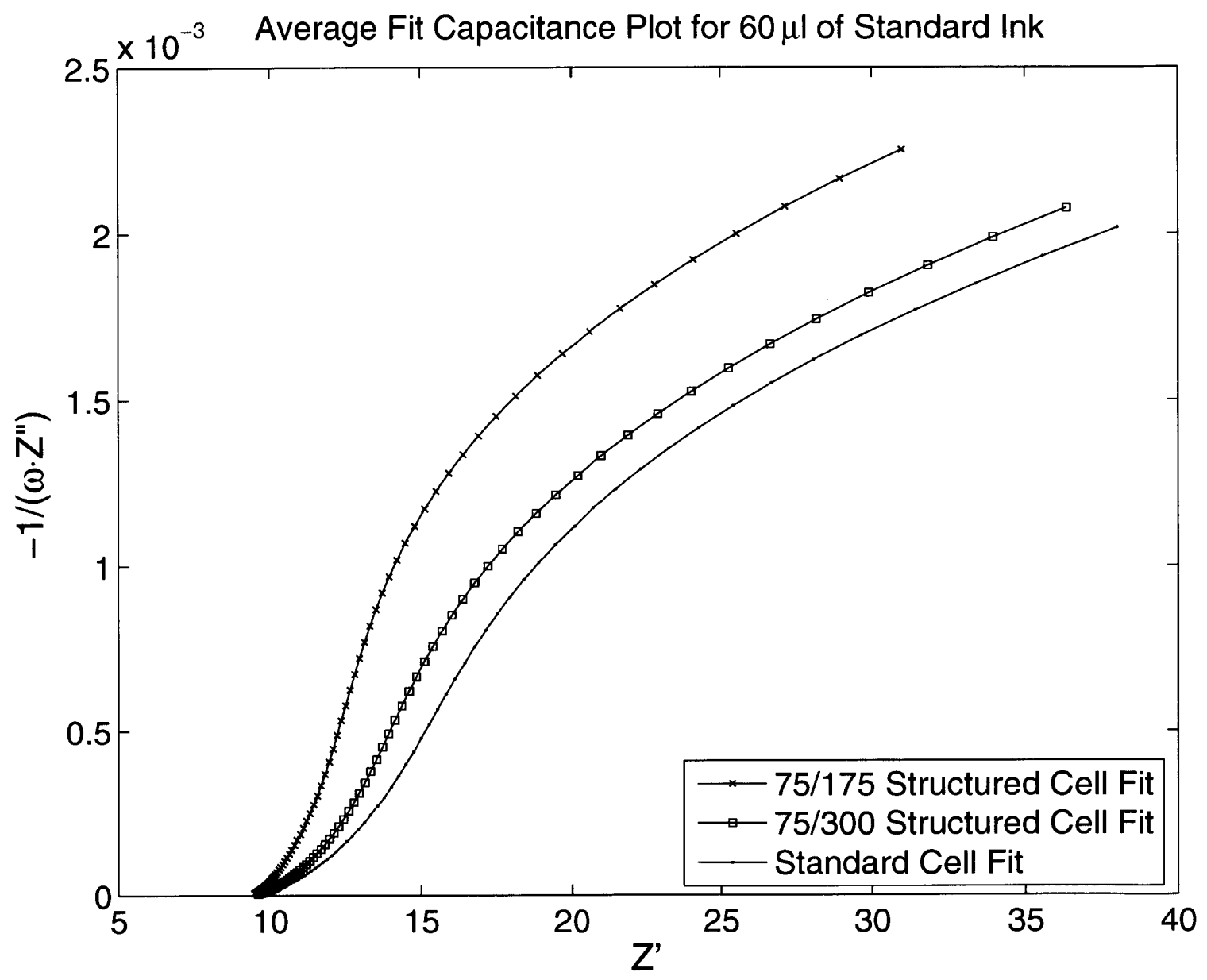

Figure 5-17: Capacitance plots calculated from the average best-fit parameters of structured and unstructured electrodes made with $60 \mu \mathrm{L}$ of standard ink. The spectra have been normalized to a single series resistance value. 
Table 5.5: Average best fit parameters for structured and standard cells with 60ul low-Nafion ink using a modified transmission line model.

\begin{tabular}{|cc|c|c|c|c|}
\cline { 3 - 6 } \multicolumn{1}{c|}{} & $R_{s}$ & $R$ & $T$ & $\phi$ \\
\hline \hline $75 / 175$ & Mean & 8.858 & 19.167 & 0.0025 & 0.774 \\
\cline { 2 - 6 } & St. Dev. & 2.584 & 2.513 & $3.95 \cdot 10^{-4}$ & 0.024 \\
\hline $75 / 300$ & Mean & 7.499 & 9.426 & 0.0027 & 0.784 \\
\cline { 2 - 6 } & St. Dev. & 1.536 & 0.570 & $4.00 \cdot 10^{-5}$ & 0.004 \\
\hline \multirow{2}{*}{ Std. } & Mean & 6.764 & 16.719 & 0.0022 & 0.713 \\
\cline { 2 - 6 } & St. Dev. & 0.811 & 11.431 & $3.99 \cdot 10^{-4}$ & 0.038 \\
\hline
\end{tabular}

plot. The structured cells have a decidedly larger capacitance term with the $75 / 175$ structured cell's value being slightly lower than that of the $75 / 300$ cell. Adding structure to an electrode with low-Nafion content can thus improve available catalyst area by more than $20 \%$.

\subsubsection{Influence of Structured Electrodes on Catalyst Loading Limits}

Adding ionically conductive structure to an electrode shows clear benefits by increasing available catalyst area. As more catalyst is added to the electrode, the improvement from adding posts should increase, since the additional catalyst would increase ionic resistance in the standard electrode more than in a structured electrode with ionically conductive channels. However, structured electrode performance also decreases after the catalyst loading limit has been reached. Figure 5-19 shows the impedance spectra of structured and standard electrodes made with $80 \mu \mathrm{L}$ of standard Nafion ink. Recall from Figure 5-10 that $80 \mu \mathrm{L}$ of standard ink was the loading at which electrode performance first decreased with an increase in catalyst. Based on the low-frequency asymptote, the structured cells perform only slightly better than the standard cell and decidedly worse than the $60 \mu \mathrm{L}$ electrodes, both structured and unstructured, shown in Figure 5-17. The modified transmission line fit data in Table 5.6 confirm these observations.

The reasons for this departure from expectation are unclear. One likely scenario is that the geometry of the structures used in these experiments are not in the ideal range to alleviate diminishing catalyst returns. The models presented in Chapter 3 are not capable of predicting the diminishing return issue in standard cells, despite the fact that this phenomenon was observed in experiments and in the literature [2]. As a consequence, the geometry for these experiments was selected based on intuition of the cell behavior. That intuition suggests 


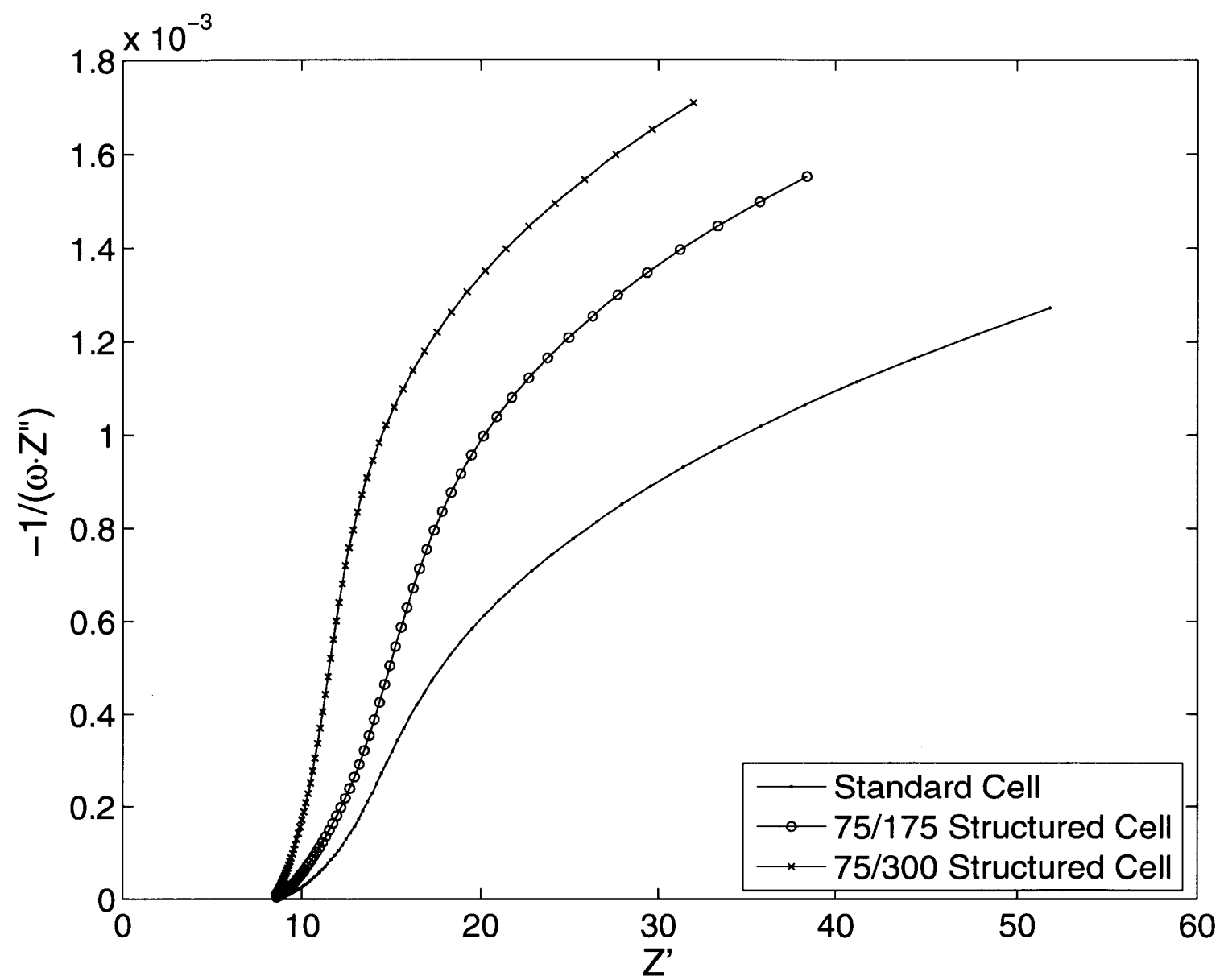

Figure 5-18: Capacitance plots calculated from the average best-fit parameters of structured and unstructured electrodes made with $60 \mu \mathrm{l}$ of low-Nafion ink. 


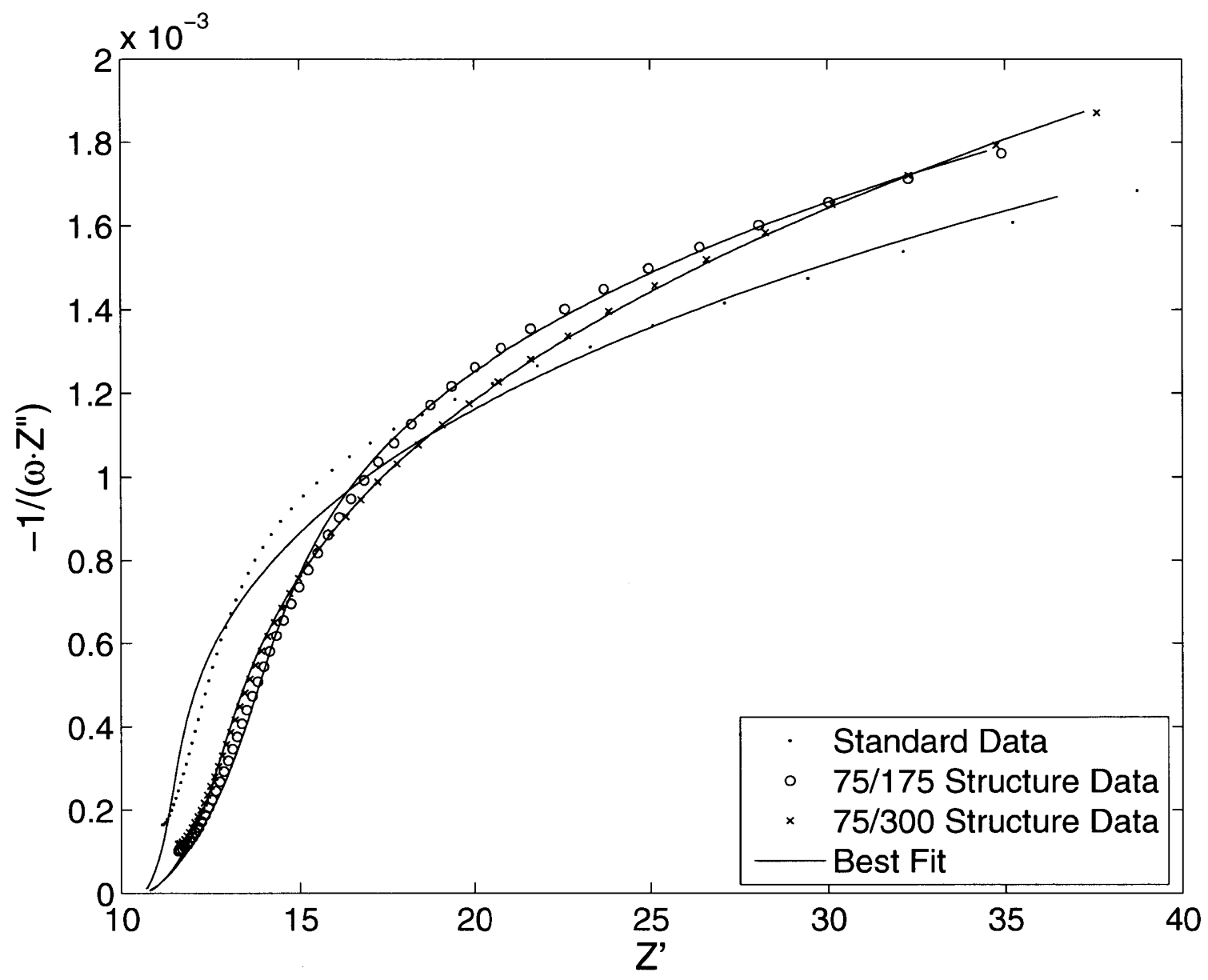

Figure 5-19: Capacitance plots of structured and standard electrodes made with $80 \mu l$ standard ink.

that densely packed posts should provide a reduction in ionic resistance in the conductive path to the largest amount of catalyst. Another possibility is that the actual geometry of the cell is not consistent with the designed intent. If, for instance, catalyst applied in the higher loading adhered to the Nafion posts near the top of the structure rather than wetting the full depth of the features, then the performance enhancement from the posts could easily be lost. Additionally, it has not yet been established that ionic conductivity is the only major driver of the observed catalyst loading limit. Further investigation is needed to establish the ideal geometry to increase the catalyst loading limit in fuel cell electrodes.

There is, however, strong evidence that a properly designed and fabricated structured electrode can eliminate the catalyst loading limit. That evidence can be found in Figure 5-13, which shows the impedance spectra of several cells using low-Nafion electrodes. As catalyst loading is increased from $20 \mu \mathrm{L}$ of ink to $60 \mu \mathrm{L}$ of ink, the amount of available catalyst, 
Table 5.6: Best fit parameters for structured and standard cells with $80 \mu l$ standard ink using a modified transmission line model.

\begin{tabular}{|c||c|c|c|c|}
\cline { 2 - 5 } \multicolumn{1}{c|}{} & $R_{s}$ & $R$ & $T$ & $\phi$ \\
\hline \hline Standard Electrode & 9.456 & 2.464 & 0.0028 & 0.747 \\
\hline 75/175 Structured Electrode & 10.955 & 9.273 & 0.0028 & 0.773 \\
\hline 75/300 Structured Electrode & 11.260 & 6.175 & 0.0032 & 0.725 \\
\hline
\end{tabular}

as represented by the low-frequency asymptote, increases to a maximum at $40 \mu \mathrm{L}$ of ink, the catalyst loading limit, and decreases as more catalyst ink is added. Because electrodes made with low-Nafion content have much higher ionic resistance, the catalyst loading limit appears with less ink than with standard cells and the impact of adding structure to the electrode is magnified. In fact, when structure is added to a low-Nafion electrode with $60 \mu \mathrm{L}$ of ink, the performance of the electrode jumps not only far above the comparable standard electrode with $60 \mu \mathrm{L}$ of ink, but it jumps above the performance of the $40 \mu \mathrm{L}$ electrode, illustrating that structure can indeed be used to get beyond the catalyst loading limit. 


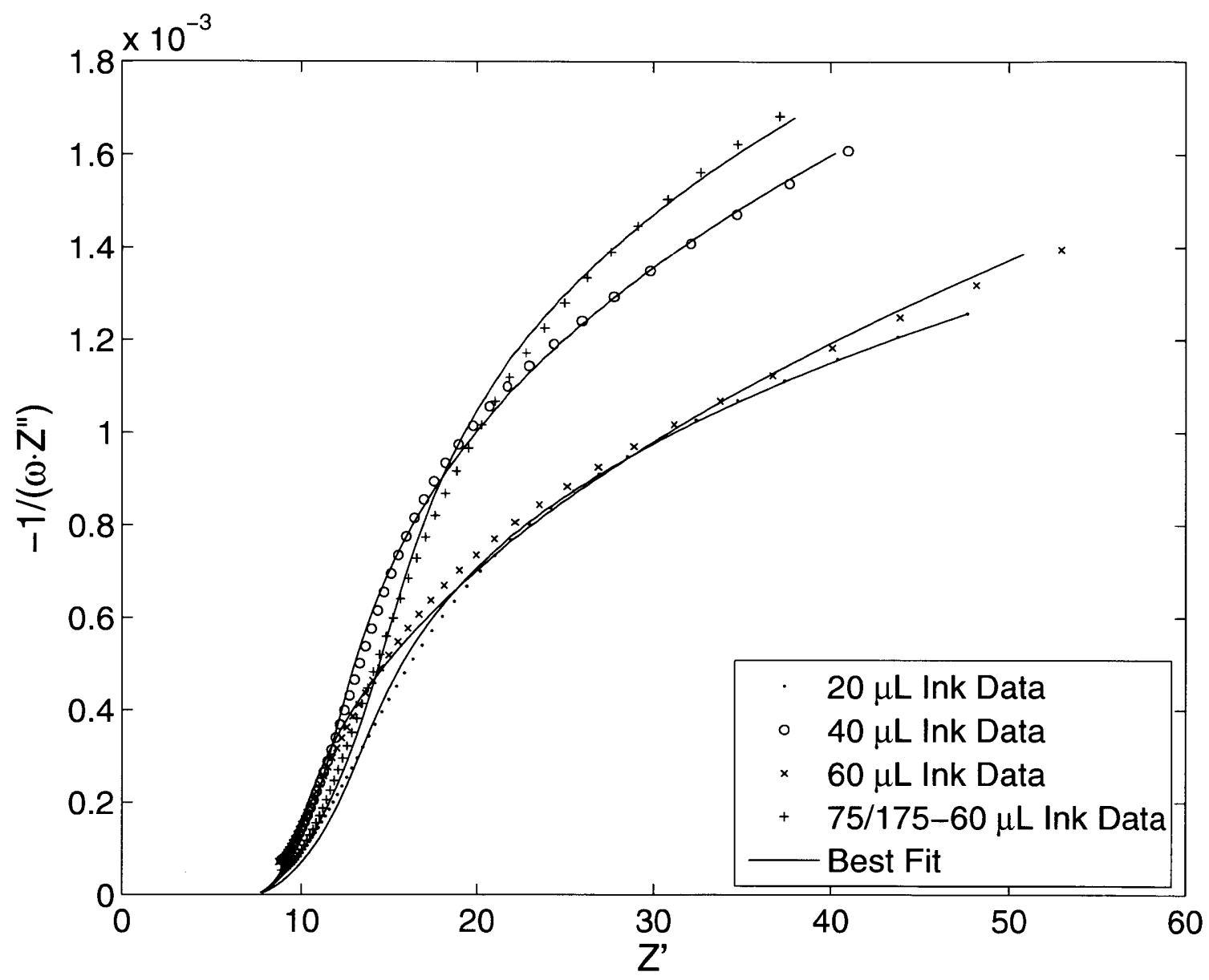

Figure 5-20: Capacitance plots of low-Nafion electrodes with increasing catalyst loading. 


\section{Chapter 6}

\section{Conclusions}

Despite being touted as the imminent replacement for lithium ion batteries, DMFCs have yet to realize the promise of long runtime in portable power applications. Commercialization of DMFCs has been plagued by a lack of sufficient power density to meet the demands of current electronics, partially due to poor anodic activation efficiency. The fact that no amount of investment, by way of catalyst loading, has been able to sufficiently address the activation issue could suggest that DMFCs are, in fact, a fundamentally flawed technology, which will never compete with lithium ion batteries. This thesis instead shows that the fundamental principles of DMFC operation are not flawed, but the current accepted design of DMFCs is the root of the power issue.

The conventional design of the DMFC anode is flawed, predominantly as a result of poor ionic conductivity within the active catalyst layer of the electrode. The use of dispersed Nafion in the electrode to control ionic conductivity is shown by Axiomatic Design analysis to be the root of a coupled design for the DMFC anode. Adding Nafion results in blockage of catalyst sites and therefore impinges on the functional requirement to provide active sites for methanol oxidation. Furthermore, this flaw cannot be avoided by adding additional catalyst, because that increases the thickness of the electrode layer and increases the ionic resistance. This is a classic example of why a coupled design is a poor design. The design has been used extensively for hydrogen fuel cells without issue, but the hydrogen oxidation reaction is a much faster reaction requiring much less catalyst than the methanol oxidation reaction. Only when the requirements become more stringent in the DMFC does the fundamental flaw in the design impose a limit on performance. This means that no amount of optimizing 
the current DMFC anode can yield sufficient performance, instead a new design is required to eliminate the coupling and allow the satisfaction of the function requirements.

The original design proposed in this thesis is a structured electrode in which ionically conductive Nafion posts are added through the thickness of the electrode to control ionic conductivity. The ionic conductivity in the structured anode is controlled by changing the post geometry and has no influence on the access to catalyst sites. Similarly, adding catalyst to the electrode does not change the post geometry and, therefore, does not change the ionic resistance of the electrode. These assertions are confirmed by the use of an original model for impedance spectroscopy of structured electrodes. The structured electrode model confirms that ionic resistance is controlled by the gap aspect ratio of the structure and, above a critical thickness, is invariant with catalyst loading. The addition of posts to the anode transforms the design from a coupled design to a decoupled design, thus allowing a design solution to be found where previously only an optimization was possible.

Confirmation of the structured electrode's superiority is found experimentally using impedance spectroscopy and cyclic voltammetry. Impedance spectroscopy experiments are performed on electrodes fabricated by embossing the desired structure onto the Nafion membrane and by then applying the electrode material between the posts. When tested as blocking electrodes, these cells exhibit behavior much like that of a transmission line with a constant phase element between the ionic and electronic conduction regimes. Consequently, ionic resistance of the electrode can be clearly seen on a Nyquist plot and the available catalyst area can be extracted from a capacitance plot.

Using structured electrodes with embossed Nafion posts results in noticeable improvements in ionic conductivity and catalyst availability. An electrode made with the ideal Nafion content of $28.6 \%$ by weight shows a $50 \%$ improvement in ionic resistance and a $6 \%$ improvement in catalyst utilization. Electrodes with lower Nafion content show even more drastic improvements in performance, with ionic resistances improving nearly $40 \%$ and catalyst utilization by over $20 \%$. These improvements are achieved without finding the optimum geometry for a particular ink mixture and catalyst loading. Increasing the gap aspect ratio of the cells from the tested value of 0.7 to the ideal value suggested by the model of 2 , could result in a $75 \%$ reduction in electrode ionic resistance. An improved model of the cell, or a more extensive set of experiments, could further improve performance by finding the optimum geometry for each particular case. 
In this thesis, no full cells were built using the structured electrode; however, it is possible to estimate the impact of the structured cell on the power output of a full cell. The polarization plot of a fuel cell can be described as:

$$
E(i)=\epsilon_{0}+b \cdot \ln \left(\frac{i_{0}}{i}\right)-R \cdot i+c \cdot \ln \left(1-\frac{i}{i_{\text {lim }}}\right)
$$

where $E$ is the cell potential, $i$ is the current density of the cell, $\epsilon_{0}$ is the equilibrium potential of the reaction, $i_{0}$ is the exchange current density, $R$ is the total cell resistance, $i_{\text {lim }}$ is the limiting current density, and $b$ and $c$ are constants. The plot of a polarization plot for a current DMFC based on this equation and using data taken from Shultz et al [40] is shown as the solid line in Figure 6-1. Assuming that electrode ionic resistance accounts for $40 \%$ of total cell resistance, the best performing structured cells could be expected to reduce cell resistance, $R$, by $20 \%$. The $6 \%$ increase in catalyst area corresponds to a $6 \%$ increase in exchange current density, $i_{0}$. The expected polarization curve for a structured cell can be calculated from this data and is shown as the dashed line in Figure 6-1. The performance of the ideal geometry for the structured model is calculated in a similar fashion and is shown as the dash-dotted line in Figure 6-1. The polarization plot can be converted into a plot of cell power density by multiplying the cell potential by the cell current density. The power density for these three cells is plotted in Figure 6-2. The best structured cell tested is estimated to improve maximum cell power by $5 \%$ while the ideal geometry could improve cell power by up to $9 \%$.

Simply increasing the cell's power density is not the main benefit of adopting the structured electrode design. The real change is the elimination of the coupling between ionic resistance and catalyst loading that limits the maximum catalyst loading of the cell. Experimentally, this was difficult to demonstrate and the catalyst loading limit was still present in cells using the ideal Nafion content of $28.6 \%$. This is likely a result of poor fabrication of cells with large catalyst loading. In cells with low-Nafion content, $9.1 \%$ by weight, the catalyst loading limit occurs at a lower catalyst loading and the electrode is much more responsive to the addition of Nafion posts. In this case, the addition of Nafion posts in the electrode is shown to eliminate the catalyst loading limit in low-Nafion content electrodes. This suggests that, with proper fabrication and more ideal structure geometry, it will be possible to eliminate the catalyst loading limit in DMFC electrodes by using structured 


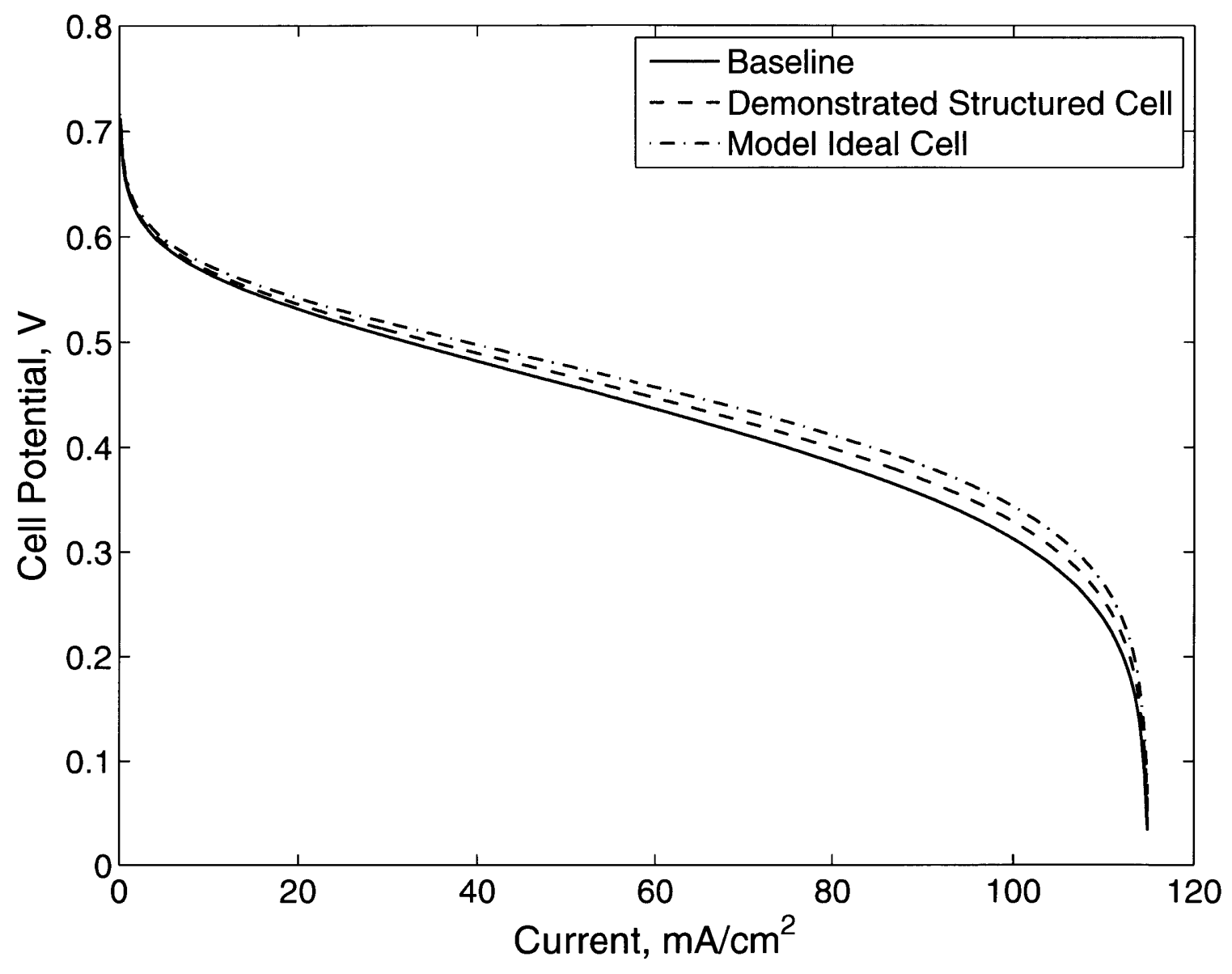

Figure 6-1: Estimated impact of structured electrodes on full cell polarization. 


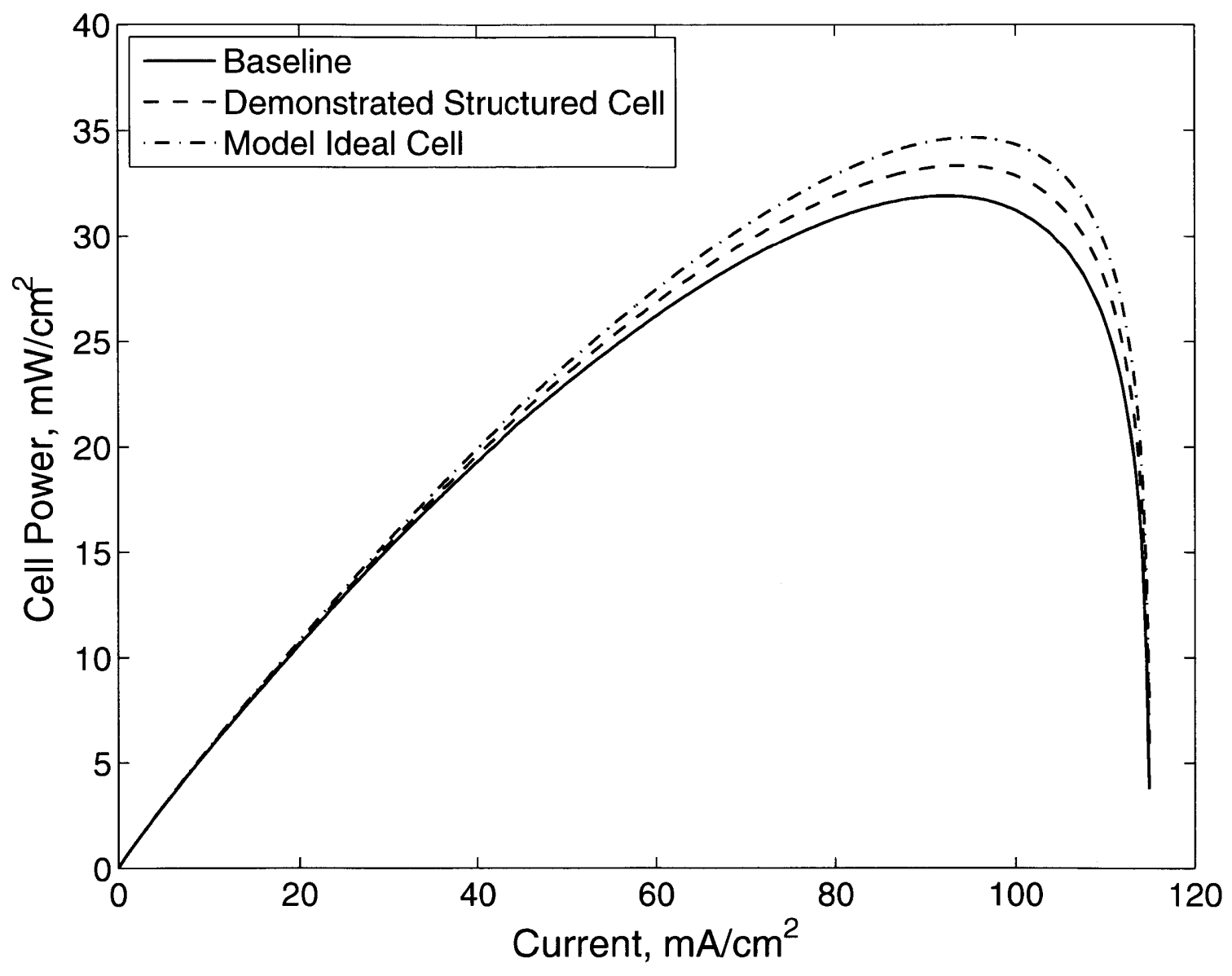

Figure 6-2: Estimated impact of structured electrodes on full cell power density. 
electrodes.

The impact of the elimination of the catalyst loading limit can be visualized using Equation 6.1 . In the case of the standard electrode, adding catalyst increases electrode thickness and therefore electrode ionic resistance. The total cell resistance is then linear in exchange current density as:

$$
R=R_{c}+\rho \cdot i_{0}
$$

where $R_{c}$ is the portion of the cell resistance not from the electrode and $\rho$ is the electrode ionic resistance per unit catalyst. In the structured electrode, the two-dimensional electrode model showed very clearly, in Figure 3-22, that ionic resistance is constant with catalyst loading so the total cell resistance, $R$, is constant with exchange current density, $i_{0}$. Figure $6-3$ shows the estimated peak power of three cells as the catalyst loading, or exchange current density, is increased. The standard cell has a maximum power just above the baseline exchange current density beyond which the additional resistance from adding more catalyst outweighs the benefit of the catalyst. The two structured cells with constant cell resistance, however, have no catalyst loading limit, and increases in catalyst loading will always improve cell power. In this situation, the other transport processes within the cell, methanol and carbon dioxide diffusion, which set the limiting current, $i_{l i m}$, would have a stronger influence on cell power than catalyst loading. Additional investigations would be required to determine how much catalyst could be added to maximize performance but, with the structured electrode, electrode ionic resistance will not limit catalyst loading as it does in current electrodes.

This thesis focused on DMFC anodes, however, the design principles that lead to the structured electrode design could also significantly improve the performance of DMFC and hydrogen-fueled PEMFC cathodes. Similar to the anodic reaction in the DMFC, the cathodic reaction in these cells is quite slow and requires large amounts of catalyst. As such, ionic resistance is a major problem and applying a structured electrode could greatly improve performance. To maximize this performance improvement, it would be necessary to design the cathode structure to meet the unique functional requirements of the cathode. In particular, PEMFC cathodes often suffer from flooding when water cannot be removed from the cathode quickly enough, so a structured cathode would need to include a mechanism to quickly remove water from the cathode, no matter how much catalyst is required. 


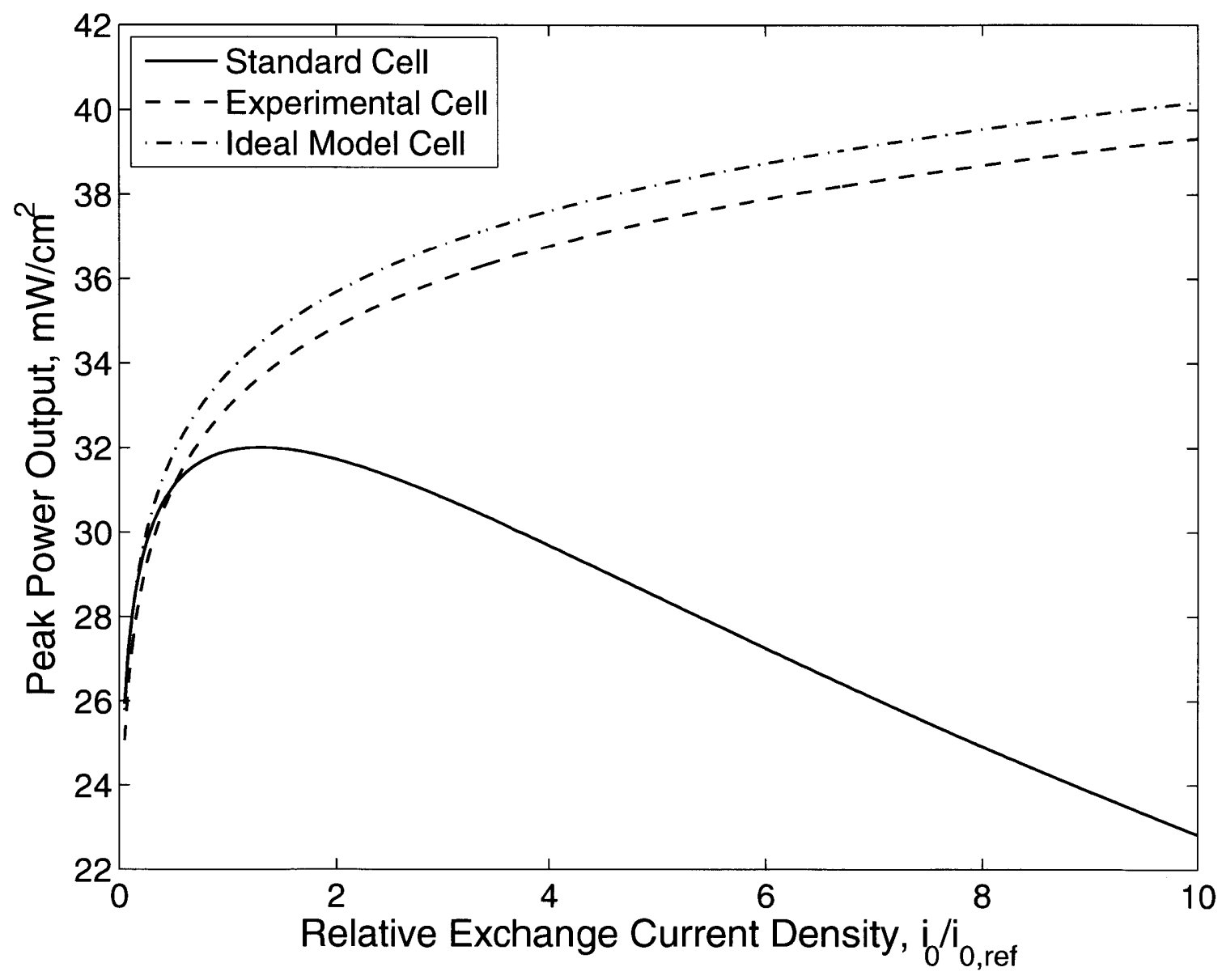

Figure 6-3: Impact of decoupled design on maximum cell power. 


\subsection{Contributions}

The main contribution of this thesis is the explanation of why DMFC electrodes must be redesigned to reach any sort of impactful performance. Section 3.1.2 describes the Axiomatic Design analysis of the current anode design and shows that functional coupling, between the requirement for catalyst area and the requirement for ionic conductivity, prevents improvement by adjusting the current design parameters. This analysis is reinforced by experimental results, presented in Chapter 5, that show the existence of a maximum catalyst loading and an ideal Nafion content.

A second, and related, contribution is the structured anode design proposed to alleviate the coupling found in the conventional anode design. The use of ionically conductive channels to allow higher catalyst loadings has never been tried before. The axiomatic motivation

for this design is presented in Section 3.2. The structured anode design is confirmed by modeling results, discussed in Section 3.4.4, which show that ionic channels can reduce ionic resistance by up to $75 \%$. Of particular importance is the result, shown in Figure 3-22, that ionic resistance of the structured design does not change with electrode thickness, and therefore, catalyst loading.

The two-dimensional impedance model, itself, is another important contribution in this thesis. The two-dimensional impedance model, described in Section 3.4.1, provides a way to evaluate the geometry of the structured electrode and determine the electrode's overall ionic resistance.

Lastly, the actual performance of the structured electrodes fabricated and tested in this thesis is significant. All structured cells performed better in experiments than an equivalent standard cell. The structured cells were able to decrease resistance by $50 \%$ and increase available catalyst by $6 \%$, compared to the best performing conventional cells.

\subsection{Future Work}

The structured electrodes produced for this thesis had gap aspect ratios of approximately 0.7 , but the structured electrode model predicted that a gap aspect ratio of 2 or greater would produce the most significant drop in electrode ionic resistance. The next step in the development of the structured electrode would be to improve the electrode fabrication process to produce electrodes with larger gap aspect ratios. Better fabrication procedures 
would also allow for the production of structured electrodes with a larger range of geometry combinations. This would allow for the confirmation of model predictions on how resistance changes with the structure geometry.

Another obvious step in the development of the structured electrode is to build and test a full cell that uses the structured electrode. This would require refining the fabrication process to emboss larger areas of the membrane with the Nafion posts. Once the structured design is implemented in a full cell, the performance estimates presented above could be validated and the value of eliminating the catalyst loading limit could be investigated while including all functions of the operating cell.

The models used in this thesis only considered the ionic resistance in the electrode and did not include other transport processes, such as methanol or carbon dioxide diffusion in the electrode. Improving the model of the structured electrode to include these processes could lead design rules to specify an ideal electrode structure based on a desired catalyst loading or power output.

While ionic resistance in the DMFC anode was the main performance limiter, methanol diffusion and carbon dioxide removal also play a role in anode performance. Since the structured electrode makes higher catalyst loadings possible, methanol and carbon dioxide transport will become the main performance limiters in the electrode. It would be insightful to extend the Axiomatic Design method used to create the structured electrode to include the satisfaction of these functions independently from the catalyst loading functional requirement. This essential involves searching for a fully uncoupled design, rather than settling for a decoupled design. 


\section{Appendix A}

\section{First Generation Solid Electrolyte Cells}

Before developing the impedance cell with a movable current collector, described in Section 4.3.2, many experiments were performed using a rigid current collector cell and inkbased counter electrodes. This section describes the fabrication and results obtained with these cells.

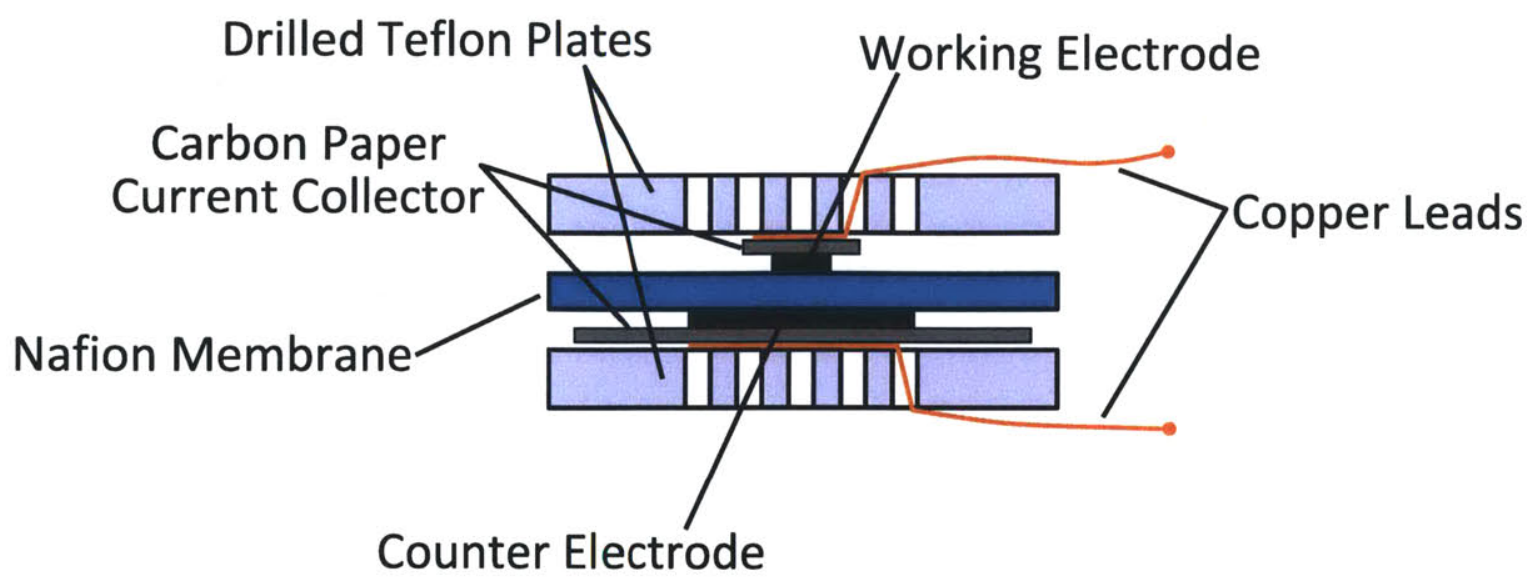

Figure A-1: Proposed impedance cell setup 


\section{A.1 Fabrication of First Generation Solid Electrolyte Cells}

\section{A.1.1 Rigid Current Collector Impedance Fixture}

The first test apparatus for the solid electrolyte cells used a rigid current collector at both the counter and working electrodes. A diagram of the assembled fixture is shown in Figure A1. The cell was sandwiched between two pieces of carbon paper that contacted directly with the electrodes on both sides. The carbon paper used behind each electrode was larger than the electrode so that it could be aligned easily, however this meant that the carbon paper would also contact the membrane in places. Behind the carbon paper on each side of the cell were $3.2 \mathrm{~mm}(1 / 8 \mathrm{in})$ thick Teflon plates each had been drilled through with 25, $3.2 \mathrm{~mm}(1 / 8 \mathrm{in})$ diameter holes. The holes allowed the humidified air to reach the membrane, ensuring that it would hydrate as quickly as possible after being placed in the high humidity environment. The cell was connected to the external electronic hardware through copper wire leads that ran through one of the drilled holes in the Teflon backing. The whole fixture was held together using binder clips.

This fixture was a vast improvement over the aqueous three-electrode cell, but it had several major drawbacks. First and foremost was the poor control of the clamp force applied to the cell. The binder clips used to hold the cell together had noticeably different stiffnesses from one another. This variation transferred into the measured impedance spectra in the cells. Another potential issue was the copper leads contacting the membrane directly. The leads were multistranded copper wire that was splayed to contact the carbon paper at more points. Poor placement of the carbon paper, however, could result in the copper contacting the membrane directly. The effect of this was not directly measured, but it seemed best to avoid the situation all together.

\section{A.1.2 Ink-Based Counter Electrode Cells}

The fabrication process for full cell using ink-based counter electrodes is shown schematically in Figure A-2. To prepare the ink-based counter electrode, the Nafion membrane was soaked in deionized water for 10-20 minutes to allow the membrane to swell isotropically. If the membrane were not pre-soaked, the water in the ink would cause the membrane to swell while clamped in place in the Teflon mask. This lead to bulges in the membrane and uneven ink application. The membrane was removed from the water and excess drops of water were 


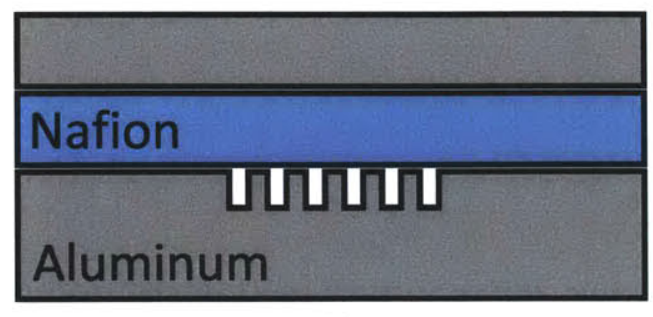

(a)

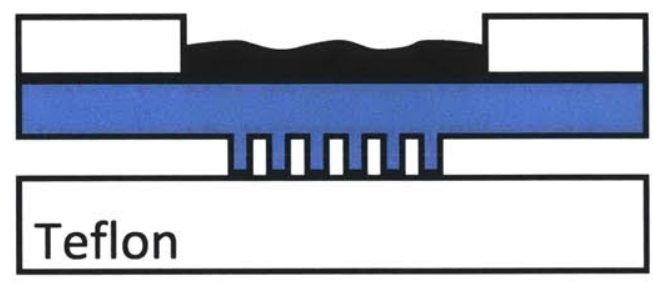

(c)

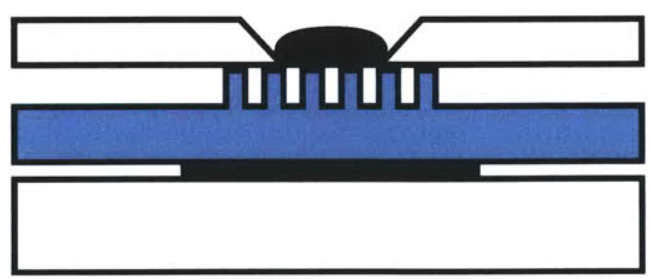

(e)

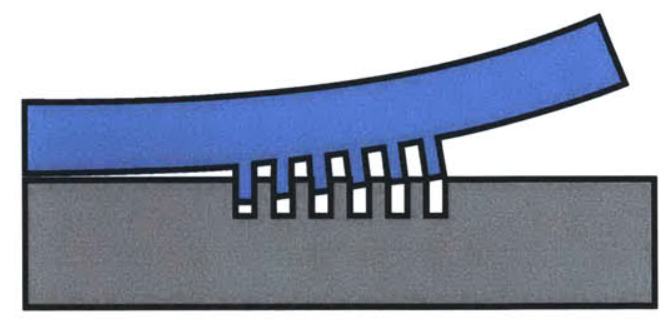

(b)

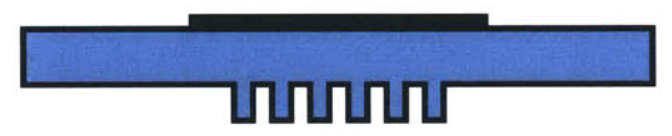

(d)

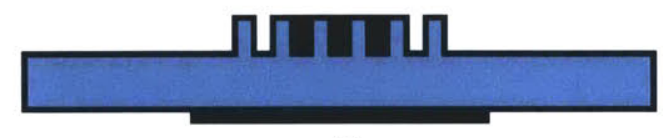

(f)

Figure A-2: Process used to fabricate structured cells with ink-based counter electrodes. 
shaken off. The membrane was then placed on a Teflon backing plate and a Teflon mask was fitted above the membrane. The Teflon mask was $3.2 \mathrm{~mm}(1 / 8 \mathrm{in})$ thick Teflon with a $25.4 \mathrm{~mm}(1 \mathrm{in})$ diameter hole milled in the center and is shown schematically in Figure A-2c. The stainless steel-Nafion-Teflon sandwich was clamped together using four binder clips. The masked membrane was then transferred to a desiccator where $1 \mathrm{~mL}$ of catalyst ink was applied to the membrane using a pipette. The electrode was then left to dry in the desiccator which took approximately 36 hours. Once the electrode was dry, the Teflon mask and stainless steel backing plate were removed.

\section{A.1.2.1 Structured Cells with Ink-Based Counter Electrodes}

The fabrication process for structured working electrodes differs slightly for cells with inkbased counter electrodes and those with carbon-paper counter electrodes described in Section 4.4.3.1. For the cells with ink-based counter electrodes, the Nafion posts were embossed onto the membrane before the counter electrode was applied. The mold to emboss the membrane was cut from a $25.4 \mathrm{~mm}$ ( $1 \mathrm{in}$ ) square piece of $1.6 \mathrm{~mm}(1 / 16 \mathrm{in})$ thick aluminum using a Microlution 363-S micromilling machine. The aluminum plate was attached to the micromill pallet using Intertape-592 double-sided tape. Unlike cutting the mold into $177.8 \mu \mathrm{m}$ $(0.007 \mathrm{in})$ thick aluminum sheet, there was no threat of punching through the $1.6 \mathrm{~mm}(1 / 16 \mathrm{in})$ aluminum plate with the drill bit. As a result, the aluminum plate could be faced more aggressively, ensuring that the full plate was planar without worrying about making it too thin in any region. The aluminum was drilled with $76.2 \mu \mathrm{m}(0.003 \mathrm{in})$ diameter holes $100 \mu \mathrm{m}$ deep with a center-to-center spacing of either $175 \mu \mathrm{m}$ or $300 \mu \mathrm{m}$.

The embossing process began by soaking the Nafion membrane in deionized water for at least 15 minutes. The membrane was then sandwiched between the micromachined aluminum mold and a flat aluminum plate. The membrane was embossed in a Carver 4386 heated press for 4 minutes at a temperature of $130^{\circ} \mathrm{C}$ under a load of $66.7 \mathrm{kN}$. The membrane was then peeled from the mold leaving Nafion posts on the surface. The ink-based counter electrode was then applied to the non-textured side of the membrane using the process described in Section A.1.2. The working electrode was then applied using the process described in Section 4.4.3.2. 


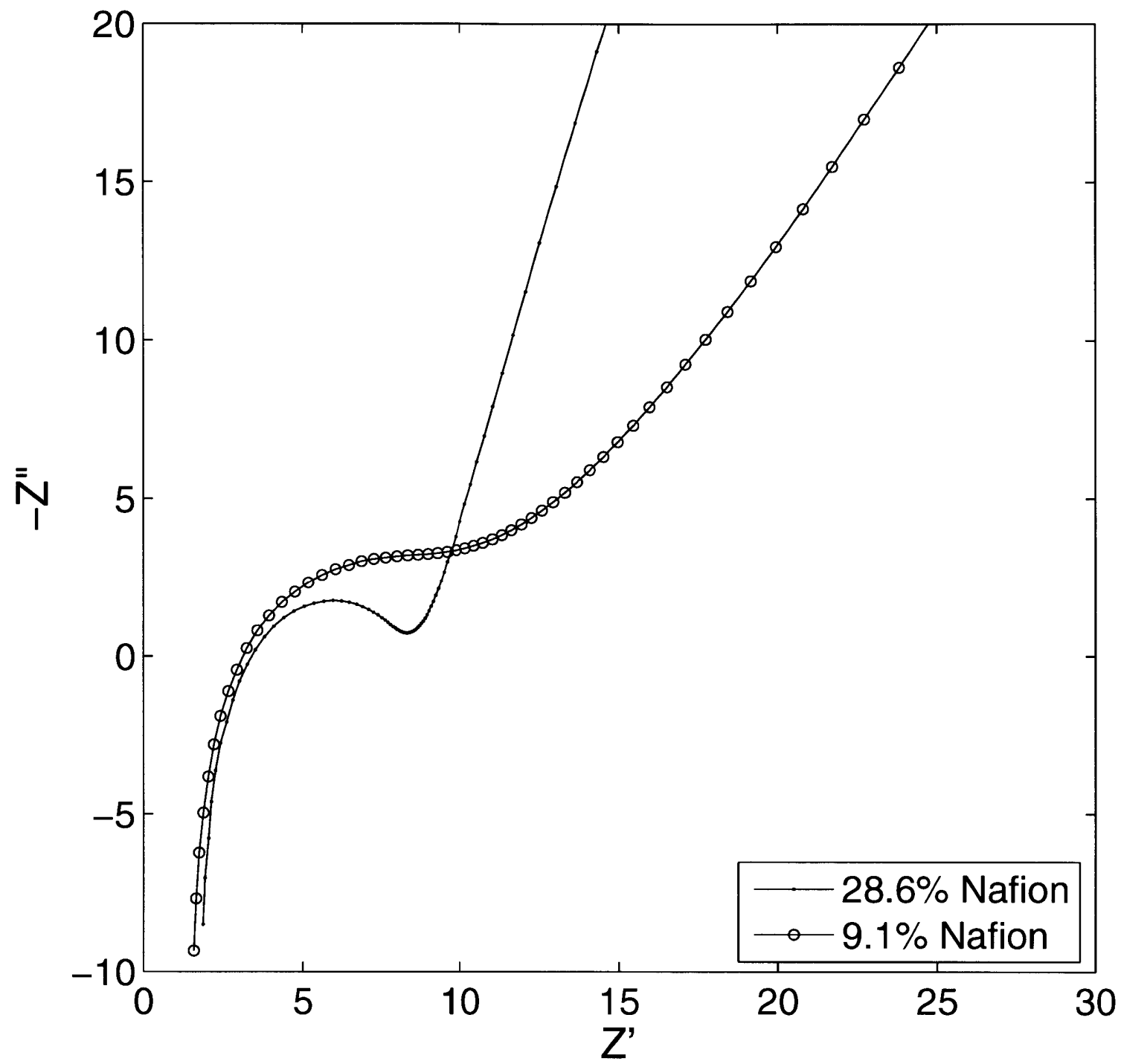

Figure A-3: First Samples with Solid Electrolyte

\section{A.2 Impedance Spectroscopy Results with First Generation Solid Electrolyte Cells}

\section{A.2.1 Resolution of Electrode Resistance}

The main goal of the impedance spectroscopy experiments are to resolve the change in ionic conductivity within the working electrode. The ability of the solid electrolyte cells to resolve changes in ionic resistance was investigated by testing cells that were identically fabricated, except that one working electrode used ink with standard Nafion loading, while the other used ink with low Nafion loading. The results of this test are shown in Figure A-3, 
Table A.1: Parallel resistor-capacitor equivalent circuit fit data

\begin{tabular}{|c|c|c|c|}
\hline & $R_{s}(\Omega)$ & $R_{p}(\Omega)$ & $C(\mu F)$ \\
\hline \hline Standard Cell & 4.020 & 3.603 & 0.730 \\
\hline Low-Nafion Cell & 3.966 & 5.837 & 0.456 \\
\hline
\end{tabular}

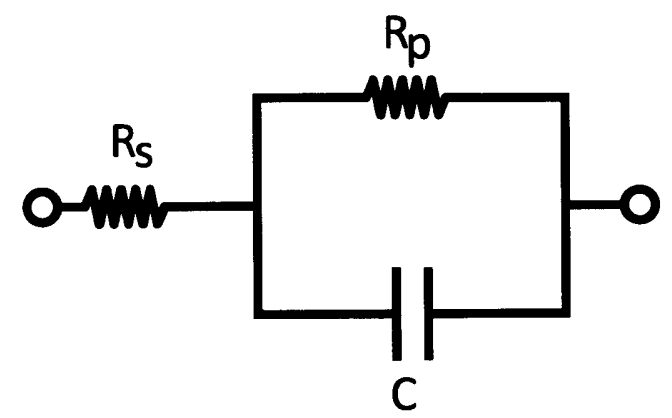

Figure A-4: Parallel resistor-capacitor equivalent circuit.

with the standard Nafion electrode plotted with dots and the low-Nafion electrode plotted with circles. The Nyquist plots of both impedance spectra show a clear semi-circle in the high-frequency region, indicative of a typical parallel resistor-capacitor circuit. In the lowfrequency region, both cells show an angled asymptote. The high-frequency semi-circle of the cell with standard Nafion loading has a noticeably smaller diameter than that of the low-Nafion cell. This indicates that the effective resistance in the standard cell is lower than that of the low-Nafion cell. This is the expected result, as the lack of Nafion in the electrode should greatly increase ionic resistance within the electrode.

Fitting just the high-frequency region to the parallel resistor-capacitor equivalent circuit shown in Figure A-4, confirms the visual interpretation of the Nyquist plot. The best fit parameters for the equivalent circuit are shown in Table A.1. Both cells have similar series resistances, $R_{s}$, which should be identical, since those values encapsulate membrane resistance and any resistance in the electrical leads. The parallel resistance, $R_{p}$, and capacitance values, however, are decidedly different. As suggested by visual inspection of the Nyquist plot, the parallel resistance value for the standard cell is smaller than that for the low-Nafion cell by more than $25 \%$. Interestingly, the capacitance value for the low-Nafion cell is significantly lower than for the standard cell. This indicates that there is less electrochemically active area within the electrode with less Nafion in the ink. This is not surprising, as less Nafion in the ink would result in fewer catalyst particles in contact with the dispersed Nafion and thus significantly less electrochemically active area. 


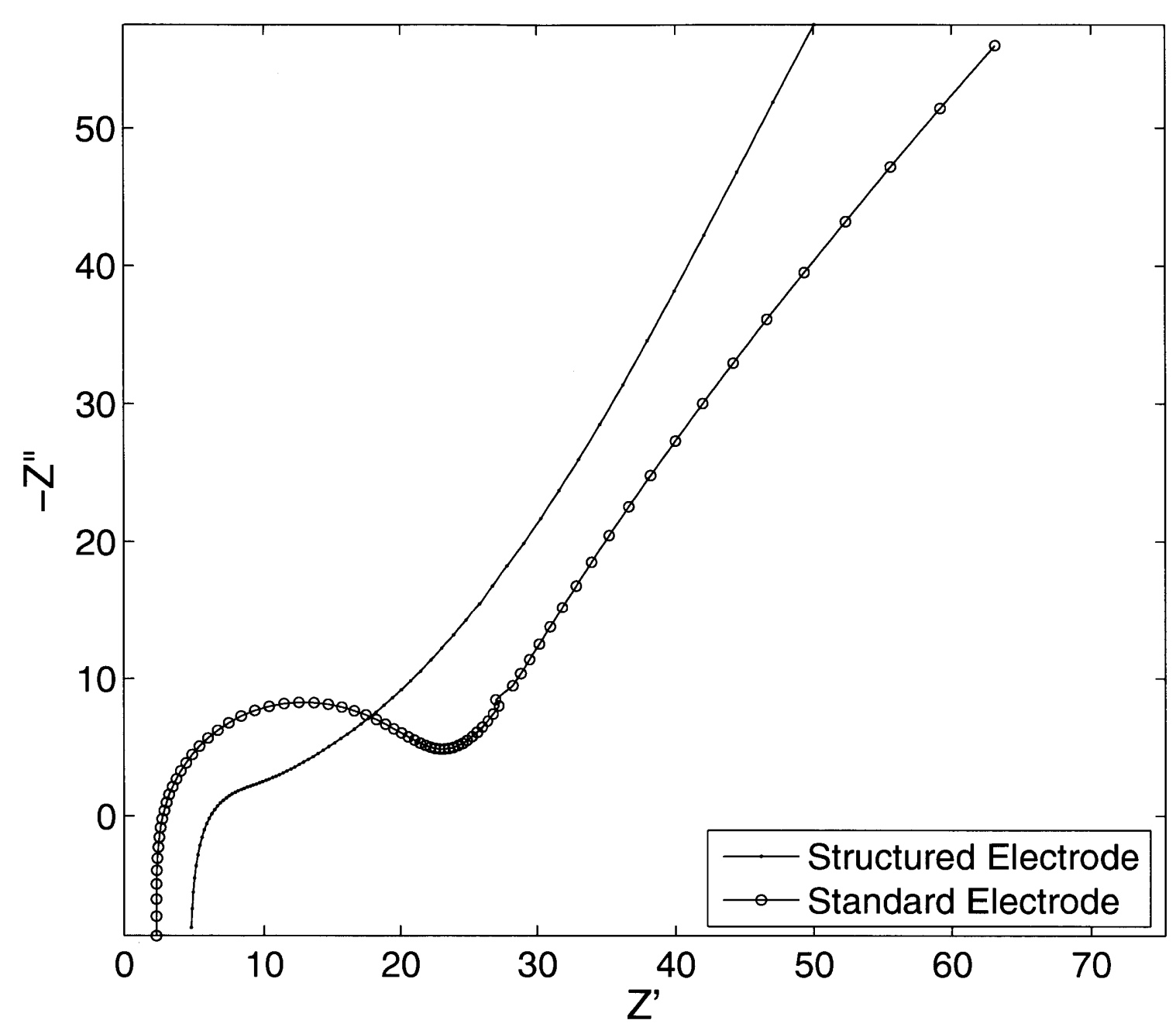

Figure A-5: Nyquist plot of a structured and a standard electrode both with ink-based counter electrodes.

\section{A.2.2 First Structured Cells}

One of the main reasons to move from the liquid electrolyte cells to those with solid Nafion electrolyte was the ability to fabricate the structured cells in the same manner as the standard cells. In the liquid electrolyte case, the structured cells required the addition of a carbon paper layer between the glassy carbon supports and the actual electrode of interest. This made direct comparisons between the two types very difficult. Using solid electrolyte, the only difference in fabrication between the types is that the solid electrolyte is embossed before the catalyst ink is applied for the structured cells. Thus, direct comparison between the cells is possible.

Figure A-5 shows a Nyquist plot of the first structured cell tested in the solid electrolyte 


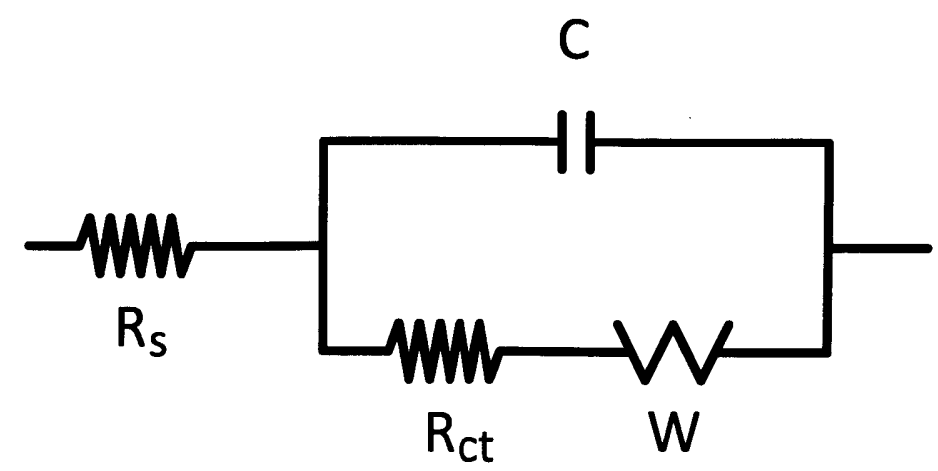

Figure A-6: Randles equivalent circuit diagram.

cell, along with a standard cell with identical catalyst loading. The structured electrode had $75 \mu \mathrm{m}$ diameter Nafion posts with a center-to-center spacing of $175 \mu \mathrm{m}$. The impedance spectra of the standard cell clearly shows the characteristic high-frequency semi-circle followed by a low-frequency mass transfer asymptote. The structured electrode, however, shows only the beginnings of the high-frequency semi-circle before approaching the low-frequency masstransfer asymptote. The poorly distinguished semi-circle in the structured cells spectra is nonetheless clearly smaller in diameter than the semi-circle in the standard cell, implying that the ionic resistance in the structured cell is significantly lower than that of standard cell. This interpretation is further reinforced when the impedance spectra are fit with a Randles equivalent circuit model, the diagram of which is shown Figure A-6. This circuit model is similar to the parallel RC-circuit, but it includes a Warburg element in resistive leg. The Warburg element is a frequency dependent element that is often used to model transport processes. It tends to dominate low-frequency behavior and leads to a $-45^{\circ}$ asymptote in Nyquist plots. The circuit parameters that best fit the two cells' spectra are show in Table A.2. The charge-transfer resistance, $R_{c t}$, would contain the electrode's ionic resistance in addition to the effective resistance as a result of change transfer at the Nafion-catalyst boundary, so it is this term that indicates the effectiveness of the electrode structure. As expected, the structured electrode shows a much lower charge-transfer resistance than does the standard cell. Additionally, the structured electrode returns a notably higher capacitance than the standard electrode, indicating that more catalyst is available for use as a result of the electrode structure. 
Table A.2: Randles equivalent circuit fit parameters for a structured and a standard cell.

\begin{tabular}{|c|c|c|c|c|}
\hline & $R_{s}(\Omega)$ & $R_{c t}(\Omega)$ & $C(\mu F)$ & $W\left(\frac{\sqrt{s}}{\Omega} \cdot 10^{-3}\right)$ \\
\hline \hline Structured Electrode & 6.79 & 3.80 & 1.26 & 1.90 \\
\hline Standard Electrode & 3.32 & 15.65 & 0.457 & 1.98 \\
\hline
\end{tabular}

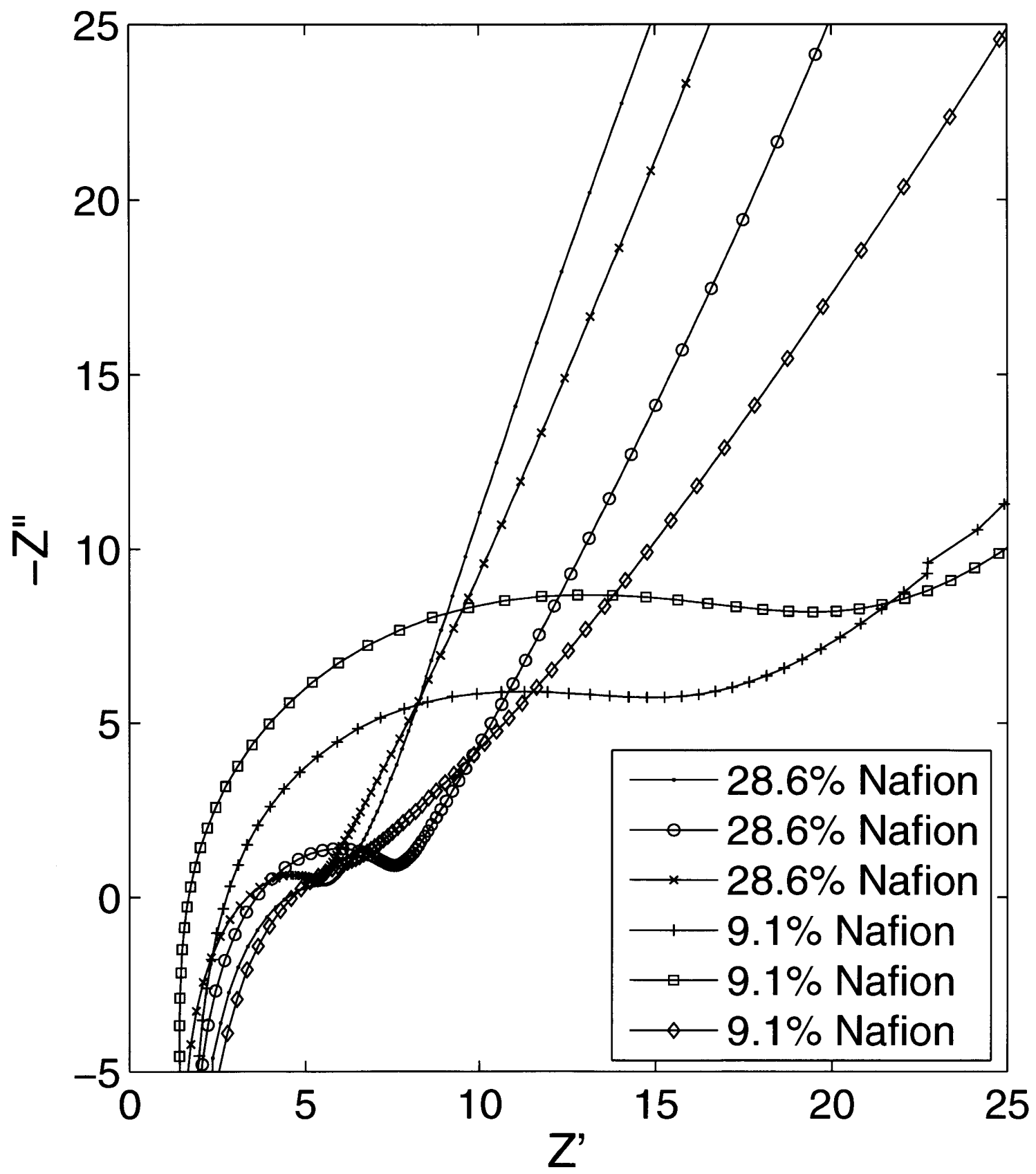

Figure A-7: Nyquist plot of cells fabricated to check cell-to-cell variation. 
Table A.3: Parallel resistor-capacitor equivalent circuit fit parameters for the data in Figure A-7.

\begin{tabular}{|c|c|c|c|}
\hline & $R_{s}(\Omega)$ & $R_{p}(\Omega)$ & $C(\mu F)$ \\
\hline \hline Standard Cell - A & 5.060 & 0.937 & 4.70 \\
\hline Standard Cell - B & 3.847 & 2.085 & 0.34 \\
\hline Standard Cell - C & 3.711 & 0.957 & 0.0083 \\
\hline Low Nafion Cell - A & 3.502 & 11.820 & 0.41 \\
\hline Low Nafion Cell - B & 2.142 & 16.250 & 0.57 \\
\hline Low Nafion Cell - C & 5.305 & $1 \cdot 10^{-6}$ & 0.93 \\
\hline
\end{tabular}

\section{A.2.3 Cell Variations}

Based on the results in Figures A-3 and A-5, it is clear that this cell apparatus with a solid electrolyte can be used to resolve differences in ionic resistance between cells. However, the unintended variation between cells was not quantified, so it was not possible to say with confidence that the resistance variation observed is due to the change imposed on the working electrode ink and not due to variations in the other parts of the cell. To determine the cell-to-cell variation, three cells were fabricated at each of the two Nafion loading levels used for the initial experiment. The Nyquist plots of the impedance spectra for these six cells are shown in Figure A-7. From the initial experiments, it was expected that the three cells with standard ink would have smaller diameter semi-circles in the high frequency region of the Nyquist plot, while the three cells with low-Nafion ink would have larger diameter semi-circles in the high-frequency region of the plot. To some degree this is true of Figure A-7 and the Nyquist plots form two distinct groups. Aside from Cell C, the low-Nafion cells did show larger semi-circles than the standard cells. However, the variation between cells is clearly quite large, making it difficult to compare changes in the working electrode structure or composition. To further illustrate the poor repeatability of these cells, the best fit parameters used to fit a parallel resistor-capacitor circuit shown in Figure A-4 to these data are shown in Table A.3. Of principle interest is the parallel resistance value, $R_{p}$, which corresponds to the ionic resistance within the electrodes. The values for the standard cells are fairly close together with one slight outlier, Cell B, and the values for the low-Nafion cells are fairly close together with one outlier, Cell C. However, unlike the data in Figure A-3, the capacitance values do not back up the hypothesis that the standard Nafion cells should have larger capacitance values as a result of more electrochemically active surface area. 


\section{A.2.4 Solid Electrolyte Cells with Carbon Paper Electrodes}

It was clear from visual inspection of the solid electrolyte cells used above that inconsistency in the fabrication of the ink counter electrode was the dominant source of variation in the cells. In the interest of reducing counter electrode variation, bare carbon paper was hot-pressed, using the process described in Chapter 4, onto the membrane to serve as the counter electrode. This arrangement was intended to make the counter electrodes identical by utilizing the same commercially produced carbon paper on all cells. The counter electrode would be made significantly larger than the working electrode such that the counter electrode's overall impedance would be low compared to that of the working electrode. The initial test of these cells is shown in Figure A-8. In this experiment, three cells were fabricated to be identical with $20 \mu \mathrm{L}$ of standard ink applied as the working electrode and a $38 \mathrm{~mm}$ square piece of carbon paper as the counter electrode. A fourth cell was made with the same working electrode, but the counter electrode was a $32 \mathrm{~mm}$ square piece of carbon paper. A fifth electrode used $20 \mu \mathrm{L}$ of low-Nafion ink as the working electrode, while returning to the $38 \mathrm{~mm}$ square carbon paper as the counter electrode. The final electrode returned to the original standard working electrode with a $38 \mathrm{~mm}$ piece of carbon paper as the counter electrode. This time, however, the carbon paper had been painted with standard composition catalyst ink before being pressed onto the membrane.

If the bare carbon paper were functioning properly as a counter electrode, then several results would be expected from the impedance spectra of these six electrodes. First, the three identical electrodes should have largely similar spectra. This expectation is roughly confirmed by the results in Figure A-8. The second expected result, is that the cell with the slightly smaller counter electrode should have approximately the same impedance response as the first three. This would indicate that the counter electrode is large enough such that slight changes in the counter electrode area produce very little change in the overall cell impedance, since the counter electrode impedance should be much smaller than that of the working electrode. This expectation is somewhat confirmed by Figure A-8, in that the

impedance spectra of the small counter electrode is largely similar to that of the other cells, except that the low-frequency tail turns away from the real axis sooner than the original three cells. The final expected result was that the cell that used low-Nafion ink should exhibit a demonstrably different behavior than the cells that use standard ink. This expectation is 


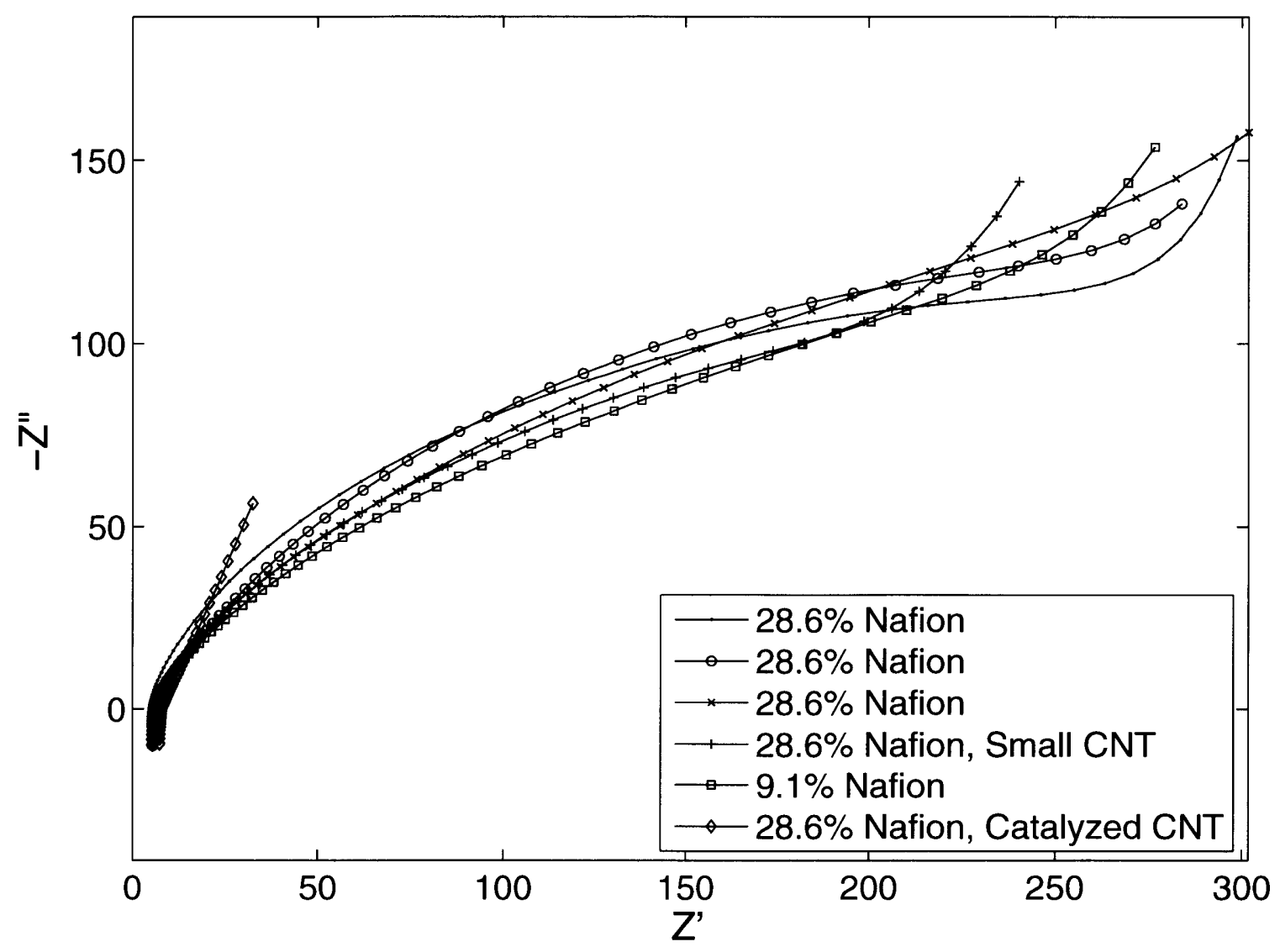

Figure A-8: Nyquist plot of cells with carbon paper counter electrodes. 
clearly violated as the cell with the low-Nafion ink falls squarely in the middle of the four cells from which it should differ.

The sixth cell in this group was added to determine if removing the catalyst from the counter electrode and using only bare carbon paper was a viable option. The cell with the catalyzed carbon paper electrode had a significantly lower impedance magnitude and it was the only cell to deviate from the five cells, despite the fact that it used the same working electrode as four of the other cells in this experiment. It is clear then, that the bare carbon paper counter electrode contributes significantly to overall impedance of the cell, making it impossible to resolve changes in the impedance of the working electrode. 


\section{Appendix B}

\section{Supplementary Results}

\section{B.1 Cyclic Voltammetry of Structured Electrodes}

Fabricating structured electrodes, such that they can be securely mounted on the glassy carbon supports used for cyclic voltammetry, proved quite difficult. The only option that seemed viable was to cast the Nafion posts onto a piece of carbon paper, then punch out a circular section and attach it to the glassy carbon electrode support with a small amount of Nafion solution. The details of this process are described in Section 4.4.2.2.

Figure B-1 shows the cyclic voltagram acquired from the cast Nafion posts on carbon paper along with the cyclic voltagram of a standard cell. Both electrodes used $15 \mu \mathrm{L}$ of $16.7 \%$ Nafion ink. It is hard to tell in Figure B-1, but the standard electrode design does exhibit very clear hydrogen adsorption peaks when the plot is zoomed to fit. The structured electrode, however, does not look anything like a typical electrode response. It has two main features that make it impossible to compare with the standard cell. First, the current drawn for the structured cell is much greater than the standard cell. The second feature of interest is that the structured electrode's voltagram has a net slope. That is, when the potential sweep switches from positive to negative at the far right of the voltagram, it takes a long time for the current to switch from positive to negative.

The larger magnitude of the current for the structured cell is most likely due to a much larger double-layer capacitance in the structured cell from the large carbon paper surface area. From the definition of a capacitor [37] 


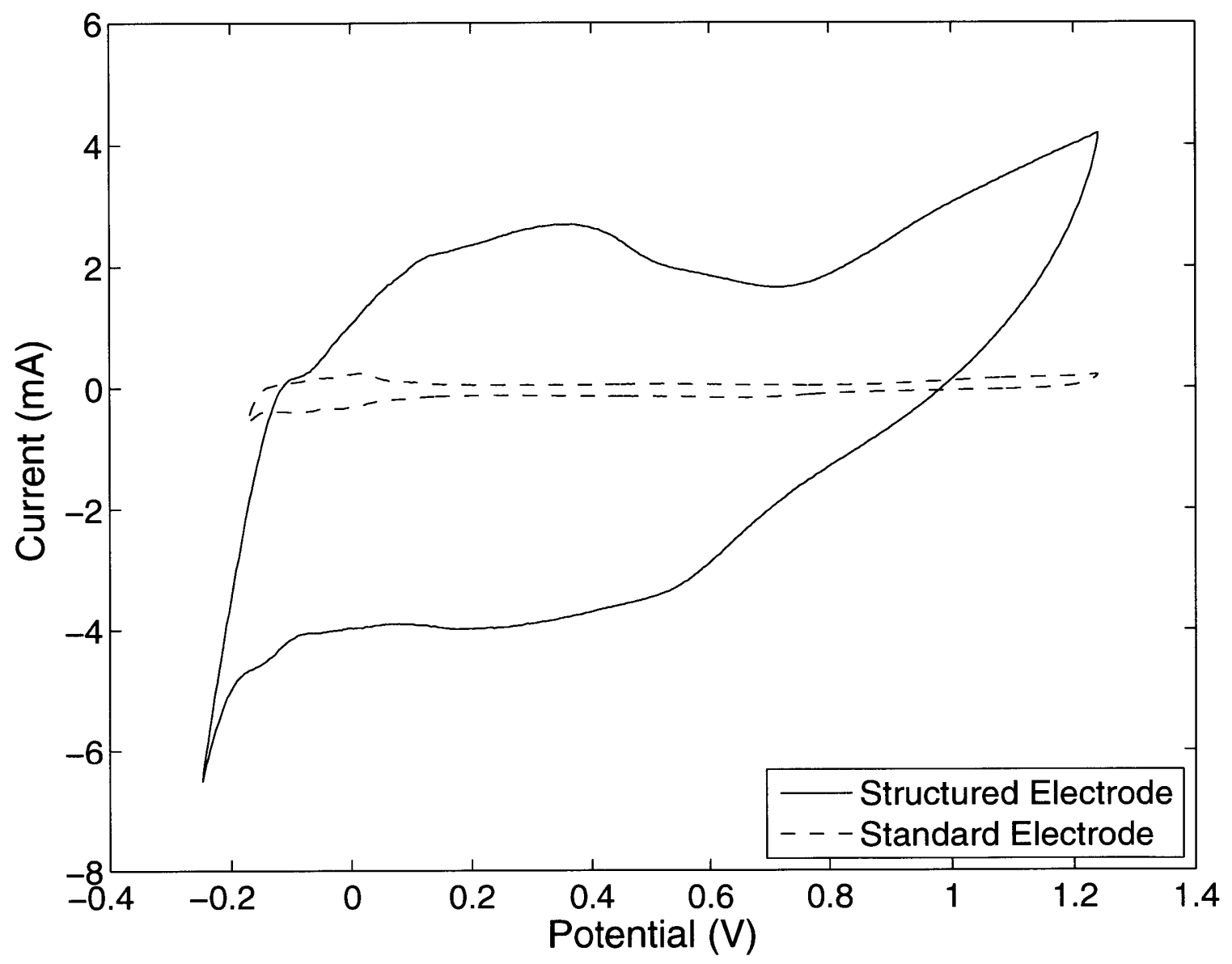

Figure B-1: Voltagram of a structured electrode and a standard electrode. 


$$
I=C \frac{\mathrm{d} V}{\mathrm{~d} t}
$$

with constant potential sweep rate, an increase in capacitance will result in an increased current. The slope of the voltagram is a result of a larger than normal resistance in the cell. The origins of this behavior are found in a simple RC-charging circuit. In these circuits, the relaxation time constant is the resistance times the capacitance. A larger resistance and capacitance results in a longer charging or discharging time. In this case, the large resistance and capacitance result in a long time required for the current to switch polarity after the potential sweep has switched.

The resistances of the individual components of the electrode are too small to account for this behavior, so the resistance must be a contact resistance between components of the electrode. The two options are between the catalyst ink and the carbon paper, where the cast Nafion may be blocking electronic contact between the paper and the carbon supports; or between the carbon paper and the glassy carbon support, where there may be too few contact points between the carbon paper and the glassy carbon to form a low resistance connection.

These two consequences of the cast Nafion design, the large double-layer capacitance and the large contact resistance, make it impossible to extract meaningful conclusions about the structured electrode using cyclic voltammetry, if the electrodes are fabricated in this fashion.

\section{B.2 Impedance Spectroscopy with Liquid Electrolyte Cells}

After it became clear that cyclic voltammetry would not be sufficient to evaluate the ionic resistance of structured electrodes, impedance spectroscopy was the most viable candidate to evaluate structured cells. Initial impedance spectroscopy experiments were performed using the same three-electrode cell with liquid electrolyte that was used for cyclic voltammetry. The sections that follow describe some of the impedance results found with this cell and how these results informed the development of the solid electrolyte cells used to get meaningful results for structured electrodes.

The setup of the cell is identical to that of the cyclic voltammetry experiments described in Section 4.3.1, but there is no purge gas used during the experiment, because a nitrogen connection is not available where the impedance equipment is located. Instead, the elec- 
trolyte is purged with nitrogen for about 30 minutes and then capped and brought to the lab for impedance experiments. This is not ideal, as oxygen has ample chance to dissolve into the electrolyte as it is poured into the reaction flask. The impedance analyzer did not have a potentiostat in in series to drive the cell, so the reference electrode and the counter electrode leads are connected to one another and are attached to one terminal of the impedance analyzer. The lead for the working electrode is attached to the other terminal.

\section{B.2.1 Structured Electrodes Cast on Carbon Paper}

The primary motivation for moving from cyclic voltammetry to impedance spectroscopy was the ability to separate out different impedance sources with a cell. The cyclic voltagram of the structured electrode in Figure B-1, for example, exhibited much larger capacitance and overall resistance than the corresponding standard electrode. More importantly, there is no way to determine with confidence what individual changes led to the extremely different voltagram. Impedance spectroscopy offers the possibility of parsing impedance contributions and isolating the details of interest, internal ionic resistance and available catalyst area, from the consequences of electrode fabrication, contact resistance at the electrode support and double-layer capacitance.

Figure B-2 shows the impedance spectra of two electrodes each made with standard Nafion ink taken over a scan range of $1 \mathrm{~Hz}$ to $1 \mathrm{MHz}$. One electrode is a standard electrode made by drying catalyst ink on a glassy carbon electrode support. The second is a structured electrode made by casting Nafion posts onto a piece of carbon paper, then attaching the carbon paper to an electrode support and drying ink onto the Nafion posts. Both electrodes exhibit some noise in the high-frequency range. The cells show some slight differences in behavior in the low-frequency asymptote with the structured cell having a noticeably larger impedance magnitude in the lowest frequency range. There is, however, no clear-cut distinction between these cells that can be used to compare the electrode performance using the Nyquist plot.

If the same impedance spectra are plotted as a Bode diagram, as in Figure B-3, there is one feature of note with regard to the design of these cells. In the Bode plot, most of the changes in behavior take place at the lowest frequency range, from 1 to $100 \mathrm{~Hz}$, where impedance is more challenging to measure. This means that experiments take longer and the important data is taken from the most uncertain region of the plot. This is again 


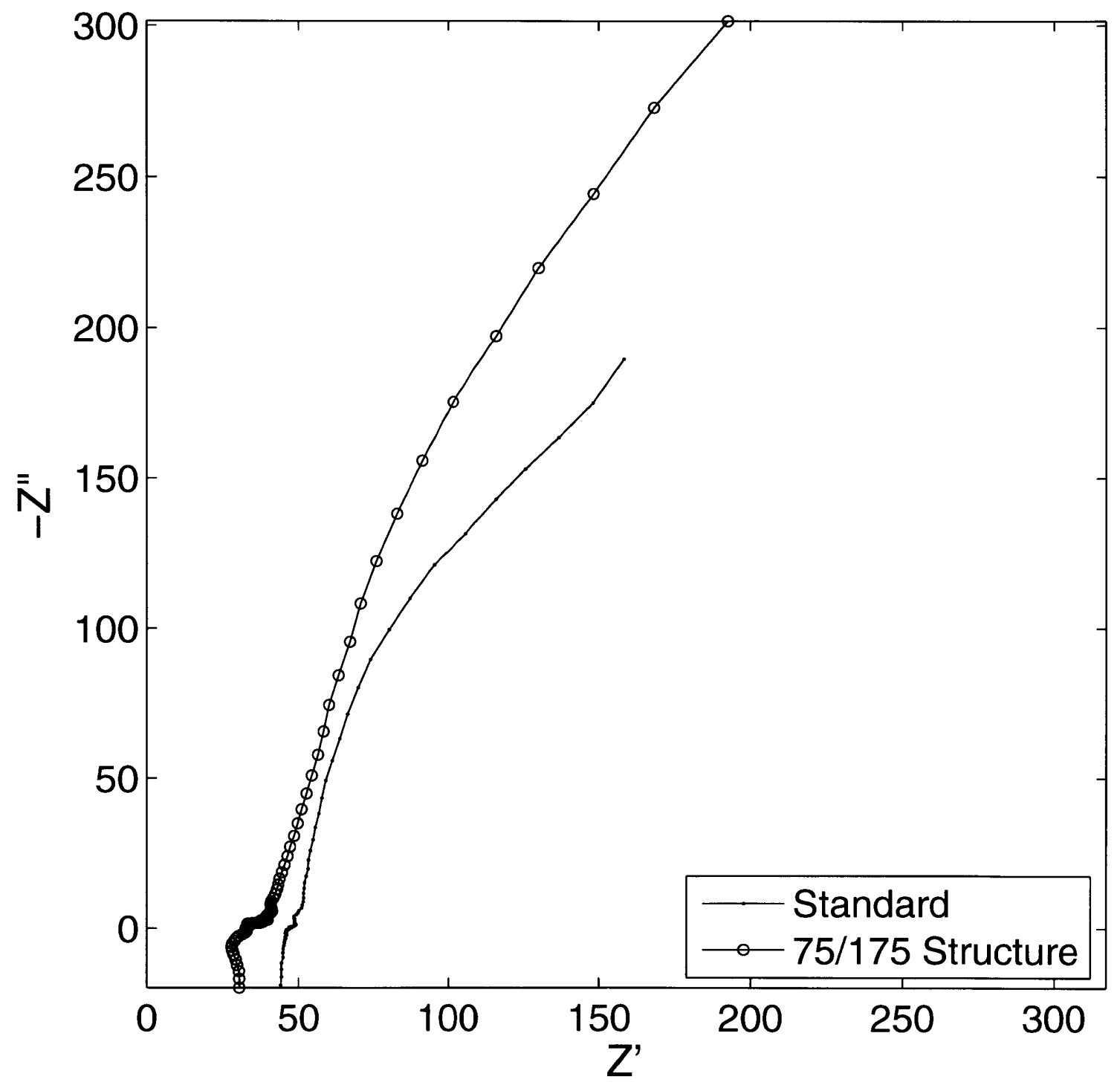

Figure B-2: Nyquist plot of standard and structured electrodes measured in the liquid electrolyte cell. 

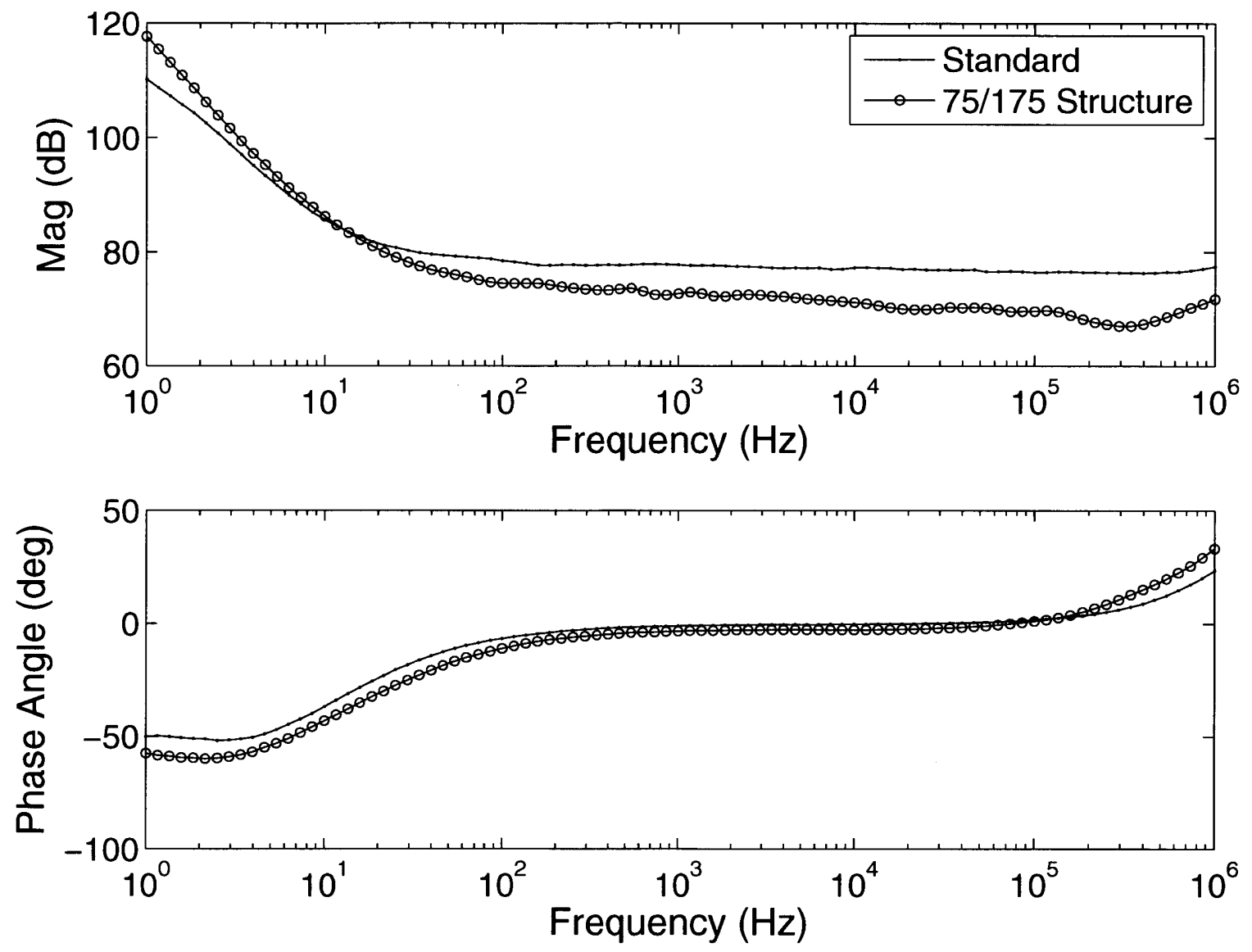

Figure B-3: Bode plot of standard and structured electrodes measured in the liquid electrolyte cell. 
a consequence of high resistance and capacitance values that result in a larger $\mathrm{RC}$ time constant.

\section{B.2.2 Nafion loading in Standard Electrodes}

Setting aside the results from the structured electrodes, the question remains, is it possible to resolve changes in ionic resistance within an electrode? To investigate this, electrodes were fabricated using inks with an increasing Nafion content, similar to those used in Figure 5-1. The impedance spectra for these cells are shown in Figure B-4. The electrodes seem to have a consistent shape, setting aside from the $9.1 \%$ and $16.7 \%$ Nafion content electrodes, but there does not seem to be any sort of trend correlated with Nafion loading. Certainly, there is nothing that would confirm the results for cyclic voltammetry that are also confirmed in published results.

\section{B.2.3 Impedance Spectroscopy of Bare Glassy Carbon Supports}

One possible source of variation between electrodes is the impedance in the electrode supports. To investigate this, impedance spectra were measured for all six glassy carbon supports and the Nyquist plot of these spectra are shown in Figure B-5. In the ideal case, where all supports are identical, the six lines in Figure B-5 would all lie on top of one-another. It is clear that there is some variation from support to support. Electrode supports A, B, and $\mathrm{F}$ are fairly similar to one another. Electrode support D is also pretty close to the others, making this set of four the typical electrode supports. The outliers are electrode supports $\mathrm{C}$ and E. Support $\mathrm{C}$ seems to be just a slightly misshapen version of the typical supports, while support E seems a completely different structure with a semi-circular section clearly present in the high-frequency range.

Figure B-6 enlarges the Nyquist plot near the real-axis intercept in the high-frequency range. This view shows that supports $\mathrm{A}, \mathrm{B}, \mathrm{C}$, and $\mathrm{F}$ have very similar series resistance values while supports $\mathrm{D}$ and $\mathrm{F}$ have somewhat larger series resistances. As the frequency decreases, support D approaches the more typical supports, while support $\mathrm{C}$ deviates from the more typical supports, yet both show similar shape in the plot.

There is no clear correlation between these variations and the experimental results using standard electrodes to perform impedance measurements. One important note is that the magnitude of the impedance for the bare supports is an order of magnitude larger than that 


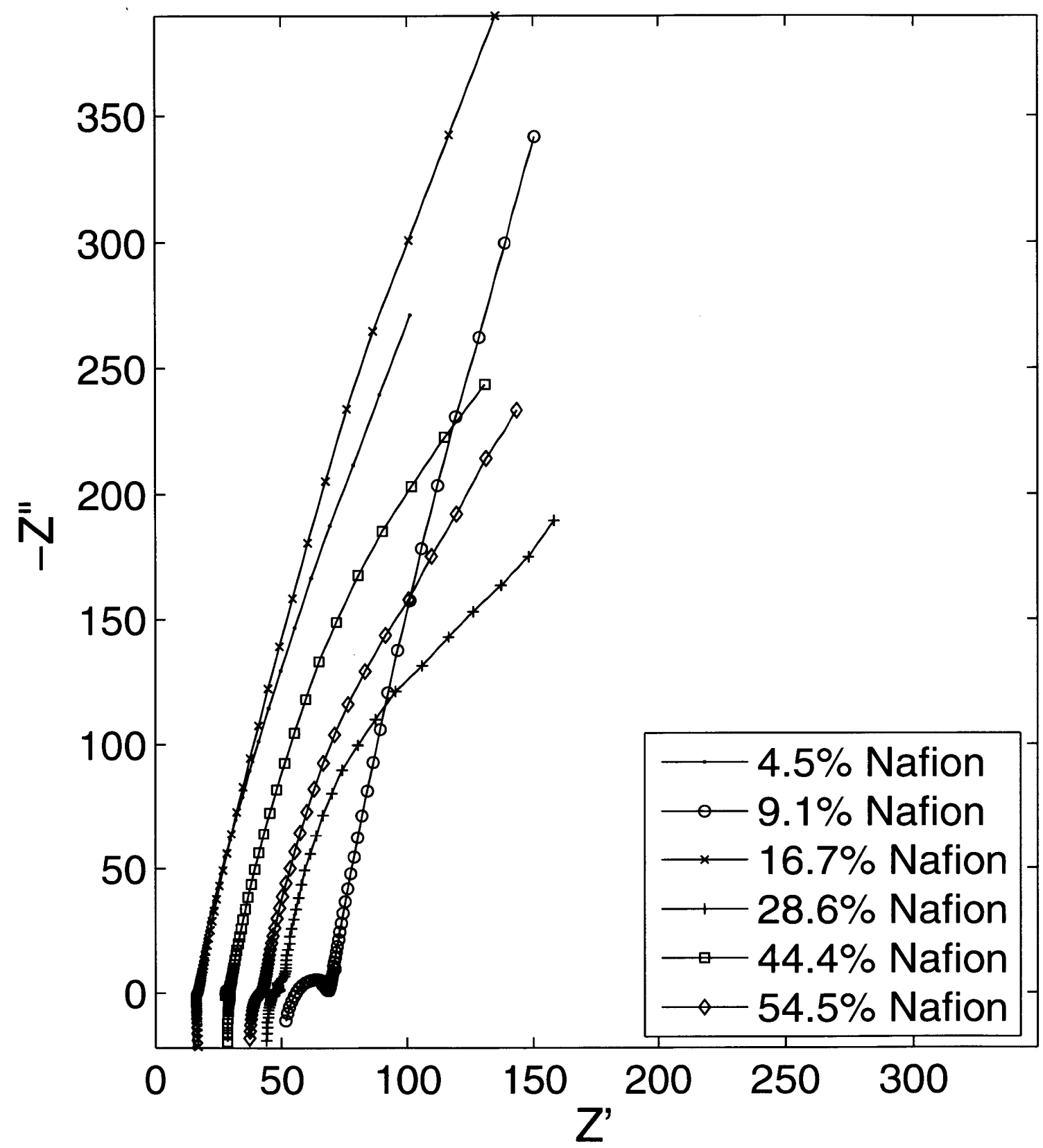

Figure B-4: Nyquist plot of standard electrodes with a range of Nafion content taken using liquid electrolyte. 


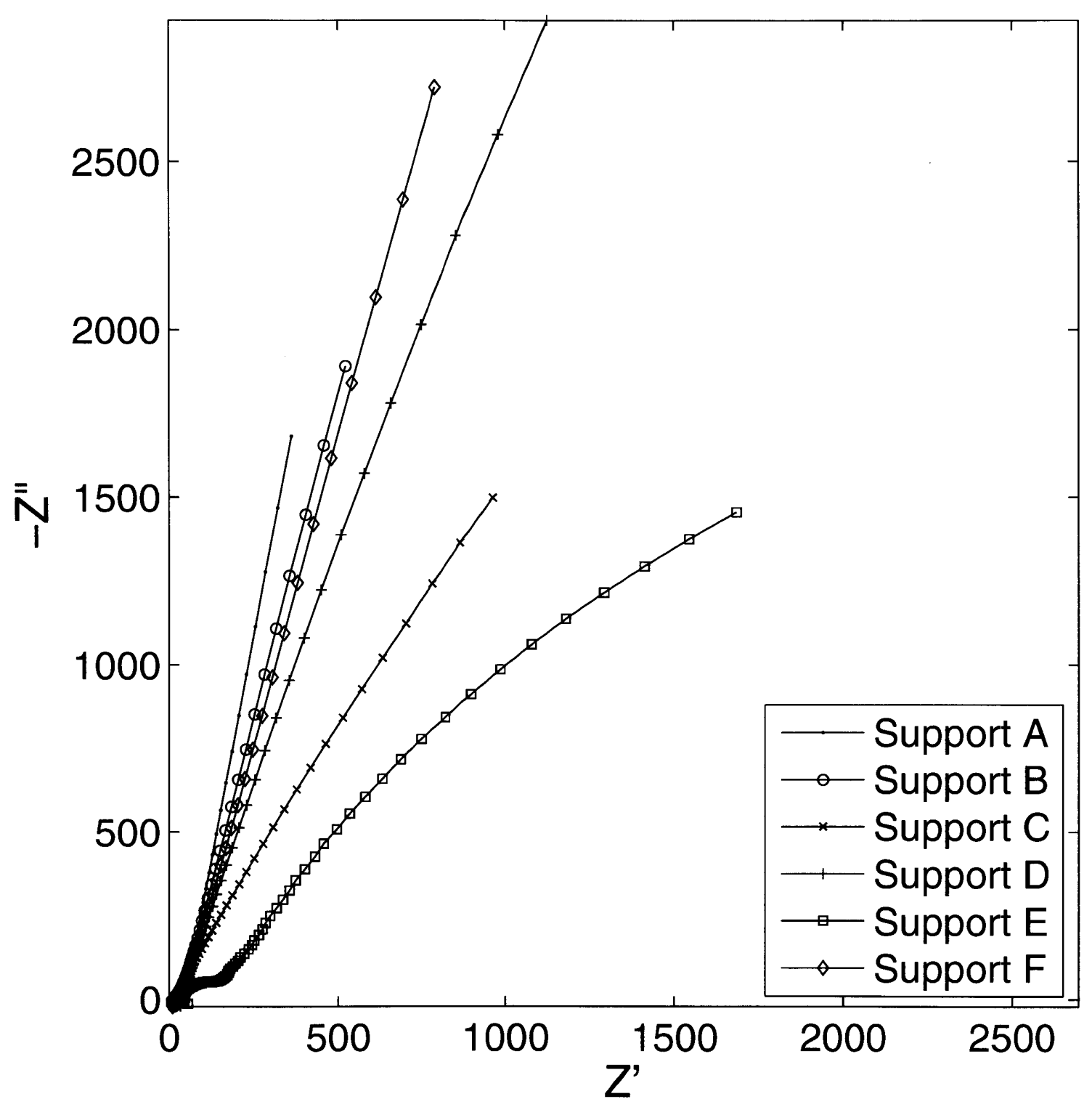

Figure B-5: Nyquist plot of impedance for all six glassy carbon electrode supports. 


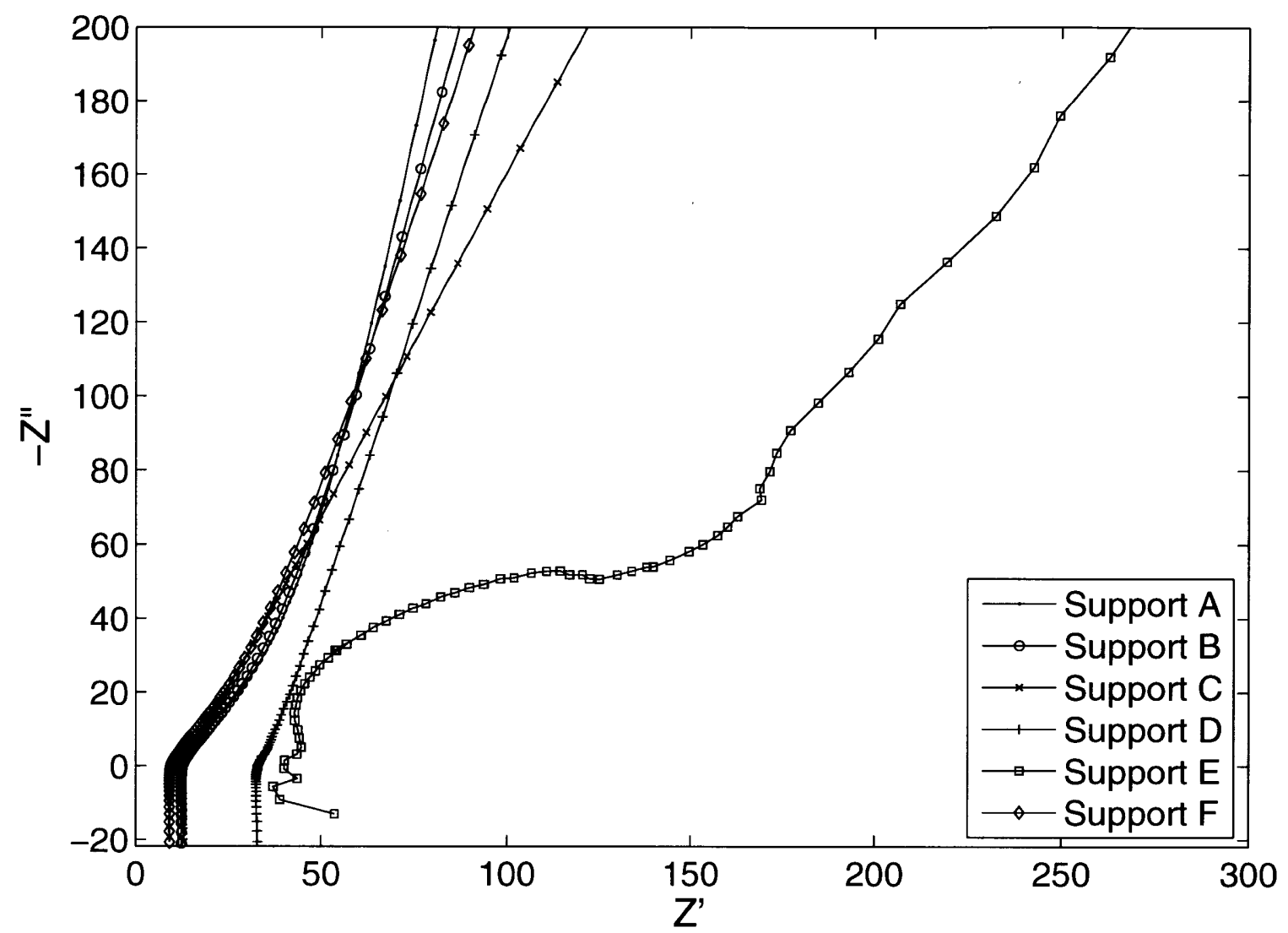

Figure B-6: Zoomed view of the Nyquist plot of impedance for all six glassy carbon electrode supports.

of the supports with a standard electrode attached. This suggests that the catalyst behavior is sufficiently dominant to hide the support influence, providing that the electrode behaves as if it is in parallel with the impedance of the electrode support.

In the end, it was determined that there were too many issues involved in impedance spectroscopy using the three-electrode cell and liquid electrolyte used with success in cyclic voltammetry. The main concern was the challenge of fabricating structured cells in such a way that they would replicate behavior in a real cell. Additionally, the inconsistent impedance of the glassy carbon electrode holders made is difficult to ensure fair comparisons between cells. The positioning of the electrodes was much more important that it was in cyclic voltammetry and the 3-electrode cell was not designed to allow for repeatable positioning. Finally the additional capacitance and resistance as a result of the carbon paper layer shifted the frequency range of interest lower, making experiments more time consuming and less reliable. 


\section{Bibliography}

[1] X. Zhao, X. Fan, S. Wang, S. Yang, B. Yi, Q. Xin, and G. Sun, "Determination of ionic resistance and optimal composition in the anodic catalyst layers of DMFC using AC impedance," International Journal of Hydrogen Energy, vol. 30, pp. 1003-1010, Aug. 2005 .

[2] T. Shimizu, T. Momma, M. Mohamedi, T. Osaka, and S. Sarangapani, "Design and fabrication of pumpless small direct methanol fuel cells for portable applications," Journal of Power Sources, vol. 137, pp. 277-283, Oct. 2004.

[3] J. Larminie and A. Dicks, Fuel Cell Systems Explained. Hoboken, NJ: John Wiley and Sons, 2003.

[4] G. Hoogers, Fuel cell technology handbook. Boca Raton, FL: CRC Press, 2003.

[5] C. Dyer, "Fuel cells for portable applications," Journal of Power Sources, vol. 106, pp. 31-34, Apr. 2002.

[6] M. Armand and J.-M. Tarascon, "Building better batteries.," Nature, vol. 451, pp. 6527, Feb. 2008.

[7] A. Hamnett, "Mechanism and electrocatalysis in the direct methanol fuel cell," Catalysis Today, vol. 38, pp. 445-457, 1997.

[8] A. Bard and L. Faulkner, Electrochemical Methods. John Wiley \& Sons, 2 ed., 2001.

[9] M. S. Wilson and S. Gottesfeld, "Thin-film catalyst layers for polymer electrolyte fuel cell electrodes," Journal of Applied Electrochemistry, vol. 22, no. 1, pp. 1-7, 1992.

[10] I. Raistrick, "Modified gas diffusion electrode for proton exchange membrane fuel cells," Diaphragms, Separators and Ion exchange Membrane, vol. 86-13, pp. 172-178, 1986. 
[11] E. Ticianelli, C. Derouin, A. Redondo, and S. Sarangapani, "Methods to advance technology of proton exchange membrane fuel cells," Journal of the ELectrochemical Society, vol. 135 , no. 9 , p. $2209,1988$.

[12] T. Springer, T. Zawodzinski, M. S. Wilson, and S. Gottesfeld, "Characterization of polymer electrolyte fuel cells using AC impedance spectroscopy," Journal of the Electrochemical Society, vol. 143, no. 2, p. 587, 1996.

[13] G. Li and P. G. Pickup, "Ionic Conductivity of PEMFC Electrodes," Journal of The Electrochemical Society, vol. 150, no. 11, p. C745, 2003.

[14] S. Srinivasan, D. Manko, H. Koch, M. Enayetullah, and A. Appleby, "Recent advances in solid polymer electrolyte fuel cell technology with low platinum loading electrodes," Journal of power sources, vol. 29, no. 3-4, pp. 367-387, 1990.

[15] E. Passalacqua, F. Lufrano, G. Squadrito, A. Patti, and L. Giorgi, "Nafion content in the catalyst layer of polymer electrolyte fuel cells: effects on structure and performance," Electrochimica Acta, vol. 46, pp. 799-805, Jan. 2001.

[16] B. Krishnamurthy, S. Deepalochani, and K. S. Dhathathreyan, "Effect of Ionomer Content in Anode and Cathode Catalyst Layers on Direct Methanol Fuel Cell Performance," Fuel Cells, vol. 8, pp. 404-409, Dec. 2008.

[17] B. Bae, B. Kho, T. Lim, I. Oh, S. Hong, and H. Ha, "Performance evaluation of passive DMFC single cells," Journal of Power Sources, vol. 158, pp. 1256-1261, Aug. 2006.

[18] M. H. Yildirim, J. te Braake, H. C. Aran, D. Stamatialis, and M. Wessling, "Micropatterned Nafion membranes for direct methanol fuel cell applications," Journal of Membrane Science, vol. 349, pp. 231-236, Mar. 2010.

[19] Y. Zhang, J. Lu, S. Shimano, H. Zhou, and R. Maeda, "Development of MEMS-based direct methanol fuel cell with high power density using nanoimprint technology," Electrochemistry Communications, vol. 9, pp. 1365-1368, June 2007.

[20] Y. Zhang, J. Lu, H. Zhou, T. Itoh, and R. Maeda, "Application of Nanoimprint Technology in MEMS-Based Micro Direct-Methanol Fuel Cell (u-DMFC)," Journal of Microelectromechanical Systems, vol. 17, pp. 1020-1028, Aug. 2008. 
[21] Z. Zhou, R. N. Dominey, J. P. Rolland, B. W. Maynor, A. A. Pandya, and J. M. DeSimone, "Molded, high surface area polymer electrolyte membranes from cured liquid precursors.," Journal of the American Chemical Society, vol. 128, pp. 12963-72, Oct. 2006.

[22] N. Suh, The Principles of Design. New York: Oxford University Press, 1990.

[23] N. Suh, Axiomatic Design: Advances and Applications. New York: Oxford University Press, 2001.

[24] K. Jeng and C. Chen, "Modeling and simulation of a direct methanol fuel cell anode," Journal of Power Sources, vol. 112, pp. 367-375, Nov. 2002.

[25] J. Newman and K. Thomas-Alyea, Electrochemical Systems. Hoboken: John Wiley \& Sons, 3 ed., 2004.

[26] O. Heaviside, Electromagnetic Theory. London: The Electrician Printing and Publishing Company, 1893.

[27] R. De Levie, Electrochemical Response of Porous and Rough Electrodes, pp. 329-397. New York: Interscience, 1967.

[28] V. Daniel-Bek, "Polarization of porous electrodes," Zh. Fiz. Khim, vol. 22, p. 697, 1948.

[29] A. Weisselberg, "Distributioin of current along a cylindrical anode inside of a concentric tube with application to chromium plating," Journal of The Electrochemical Society, vol. 90, no. 1, pp. 235-245, 1946.

[30] A. Lasia, Modeling of Impedance of Porous Electrodes, vol. 43 of Modern Aspects of Electrochemistry, ch. 3, pp. 67-137. New York, NY: Springer, 2009.

[31] T. Pajkossy, "Generalization of Basic Laws of Electrochemistry for Fractal Surfaces," Heterogeneous Chemistry Reviews, vol. 2, pp. 143-147, 1995.

[32] B. Sapoval, "Fractal Electrodes and Constant Phase Angle Response: Exact Examples and Counter Examples," Solid State Ionics, vol. 23, pp. 253-259, 1987.

[33] Z. Kerner and T. Pajkossy, "On the origin of capacitance dispersion of rough electrodes," Electrochimica Acta, vol. 46, pp. 207-211, Nov. 2000. 
[34] W. Vielstich, Cyclic Voltammetry, vol. 2, ch. 14, pp. 153-162. John Wiley \& Sons, 2003.

[35] E. Ticianelli, C. Derouin, and S. Srinivasan, "Localization of platinum in low catalyst loading electrodes to to attain high power densities in SPE fuel cells," Journal of Electroanalytical Chemistry, vol. 251, no. 2, pp. 275-295, 1988.

[36] M. E. Orazem and B. Tribollet, Electrochemical Impedance Spectroscopy. Hoboken, NJ: John Wiley \& Sons, 2008.

[37] P. Horowitz and W. Hill, The Art of Electronics. Cambridge: Cambridge University Press, 2 ed., 1989.

[38] M. C. Lefebvre, R. B. Martin, and P. G. Pickup, "Characterization of Ionic Conductivity Profiles within Proton Exchange Membrane Fuel Cell Gas Diffusion Electrodes by Impedance Spectroscopy," Electrochemical and Solid-State Letters, vol. 2, no. 6, p. 259, 1999.

[39] E. Easton and P. G. Pickup, "An electrochemical impedance spectroscopy study of fuel cell electrodes," Electrochimica Acta, vol. 50, pp. 2469-2474, Apr. 2005.

[40] T. Schultz, S. Zhou, and K, "Current status of and recent developments in the direct methanol fuel cell," Chemical Engineering \& Technology, vol. 24, no. 12, pp. 1223-1233, 2001. 